\title{
The Role of RNF157 \\ in Central Nervous System Development
}

\author{
Dissertation \\ for the award of the degree \\ "Doctor rerum naturalium" \\ of the Georg-August-University Göttingen
}

in the doctoral program:

Molecular Physiology of the Brain

of the Georg-August University School of Science (GAUSS)

submitted by

\section{Annika Matz}

born in Wilhelmshaven, Germany

Göttingen 2012

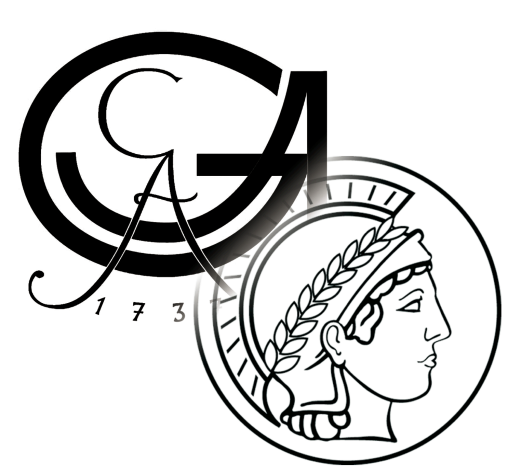




\section{Thesis Committee:}

Member of the Thesis Committee: Dr. Judith Stegmüller, Laboratory of Cellular and Molecular Neurobiology, Max-Planck-Institute of experimental Medicine, Göttingen

Member of the Thesis Committee: Prof. Dr. Tomas Pieler,

Department of Developmental Biochemistry, Center for Biochemistry and Molecular Biology, Göttingen

Member of the Thesis Committee: Prof. Dr. Anastassia Stoykova,

Department of Molecular Cell Biology, Max-Planck-Institute for Biophysical Chemistry, Göttingen

\section{Members of the extended thesis committee:}

Reviewer: Dr. Judith Stegmüller, Laboratory of Cellular and Molecular Neurobiology, MaxPlanck-Institute of experimental Medicine, Göttingen

Reviewer: Prof. Dr. Tomas Pieler, Department of Developmental Biochemistry, Center for Biochemistry and Molecular Biology, Göttingen

Reviewer: Prof. Dr. Anastassia Stoykova, Department of Molecular Cell Biology, MaxPlanck-Institute for Biophysical Chemistry, Göttingen

Prof. Dr. Detlef Doenecke, Department of Molecular Biology, Institute of Biochemistry and Molecular Cell Biology

Prof. Dr. Tomas Bayer, Department for Psychiatry, Division of Molecular Psychiatry

Prof. Dr. Michael Hörner, Department of Cellular Neurobiology, Johann-FriedrichBlumenbach-Institute for Zoology and Anthropology

Date of submission of the dissertation: $31^{\text {st }}$ of August 2012

Date of the oral examination: $11^{\text {th }}$ of October 2012 
I hereby declare that I prepared the dissertation "The Role of RNF157 in Central Nervous System Development" on my own and with no other sources or aids than quoted.

August, 2012

Göttingen, Germany

(Annika Matz) 


\section{Table of Contents}

Acknowledgements

Summary

Abbreviations $\quad$ VII

1 Introduction 1

$\begin{array}{llr}1.1 & \text { Neuronal development in the mammalian brain } & 1\end{array}$

$\begin{array}{lll}1.1 .1 & \text { Neuronal induction } & 1\end{array}$

$\begin{array}{ll}1.1 .2 \text { Neuronal proliferation } & 2\end{array}$

$\begin{array}{lll}1.1 .3 & \text { Neuronal migration } & 3\end{array}$

1.1.4 Neuronal polarization: Establishment of axon-dendrite polarity 3

$\begin{array}{lll}\text { 1.1.5 Axonal growth and guidance } & 5\end{array}$

1.1.6 Cellular and molecular mechanisms of dendrite growth and synapse formation 6

1.1.6.1 Extracellular signals in the control of dendrite growth and branching $\quad 7$

1.1.6.2 Intracellular mechanisms regulating dendrite growth and branching 8

$\begin{array}{llr}1.2 & \text { Neuronal apoptosis during development and disease } & 10\end{array}$

1.2.1 The apoptotic cell death machinery and the Bcl-2 family 11

$\begin{array}{lll}\text { 1.2.2 Programmed cell death during neuronal development } & 13\end{array}$

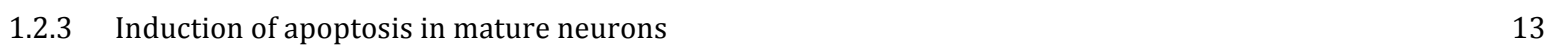

$\begin{array}{lll}\text { 1.2.3.1 Apoptotic stimuli and signaling cascades } & 13\end{array}$

$\begin{array}{ll}\text { 1.2.4 Apoptosis in neurodegenerative diseases } & 14\end{array}$

$\begin{array}{llr}1.3 & \text { Ubiquitination in neurons } & 15\end{array}$

$\begin{array}{llr}\text { 1.3.1 RING E3 ligases in focus } & 17\end{array}$

$\begin{array}{lll}\text { 1.3.1.1 RING E3 ligases in neuronal development } & 18\end{array}$

1.3.1.2 RING E3 ligases in neuronal apoptosis and neurodegenerative diseases 20

$\begin{array}{ll}\text { 1.3.2 RNF157 and its novel interactor Fe65 } & 22\end{array}$

$\begin{array}{llr}1.4 & \text { Aims of the study } & 23\end{array}$

2 Materials and Methods $\quad 24$

$\begin{array}{llr}2.1 & \text { Chemicals and reagents } & 24\end{array}$

$\begin{array}{llr}2.2 & \text { General equipment } & 24\end{array}$

\begin{tabular}{lr}
2.3 & Enzymes \\
\hline
\end{tabular}

$\begin{array}{llr}2.4 & 25\end{array}$

$\begin{array}{llr}2.5 & \text { Kits } & 27\end{array}$

$\begin{array}{llr}2.6 & \text { Vector plasmids } & 28\end{array}$

$\begin{array}{llr}2.7 & \text { Oligonucleotides } & 29\end{array}$

$\begin{array}{llr}2.8 & \text { Antibodies } & 31\end{array}$

2.9 Protein biochemistry methods $\quad 32$

$\begin{array}{lll}2.9 .1 & \text { Isolation of rodent neural and non-neural tissue } & 32\end{array}$ 
2.9.2 Preparation of rodent tissue lysates $\quad 32$

2.9.3 Preparation of HEK 293T cell lysates $\quad 32$

2.9.4 Co-Immunoprecipitation of proteins $\quad 32$

2.9.5 Subcellular fractionation of cerebellar granule neurons $\quad 33$

$\begin{array}{lll}2.9 .6 & \text { In vitro ubiquitination assay } & 33\end{array}$

$\begin{array}{lll}\text { 2.9.7 } & \text { Determination of protein concentration by Bradford } & 34\end{array}$

$\begin{array}{lll}2.9 .8 & \text { Detection of proteins by Western Blot analysis } & 34\end{array}$

$\begin{array}{ll}2.10 & 35\end{array}$

2.10.1 Perfusion of mice and generation of paraffin-embedded brain sections 35

$\begin{array}{ll}\text { 2.10.2 DAB staining of paraffin sections } & 35\end{array}$

2.10.3 Immunocytochemistry of heterologous cells and primary neurons 36

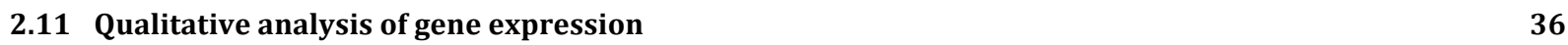

\begin{tabular}{ll}
2.11 .1 & Isolation of RNA \\
\hline
\end{tabular}

$\begin{array}{ll}2.11 .2 & \text { cDNA synthesis from total RNA }\end{array}$

\begin{tabular}{ll}
2.12 & Expression plasmid construction and cloning \\
\hline
\end{tabular}

$\begin{array}{lll}\text { 2.12.1 } & \text { General DNA construct amplification } & 37\end{array}$

$\begin{array}{ll}2.12 .2 \text { Cloning } & 38\end{array}$

$\begin{array}{lll}2.12 .3 & \text { Site-directed mutagenesis of plasmids } & 39\end{array}$

$\begin{array}{ll}2.12 .4 & \text { shRNA design and plasmid construction }\end{array}$

2.13 Culture of primary neurons or HEK 293T cells 40

$\begin{array}{lll}2.13 .1 & \text { Culture of primary cerebellar granule neurons } & 40\end{array}$

$\begin{array}{ll}\text { 2.13.2 Culture of primary hippocampal neurons } & 41\end{array}$

2.13.3 Culture of HEK 293T cells $\quad 42$

2.14 Transfection of primary neurons and HEK 293T cells $\quad 42$

2.14.1 Transfection of primary neurons $\quad 42$

$\begin{array}{ll}\text { 2.14.2 Transfection of HEK 293T } & 42\end{array}$

2.15 Analysis of neuronal morphology 43

2.16 Survival assays in neurons $\quad 43$

$\begin{array}{lll}2.17 & \text { Yeast Two-Hybrid } & \mathbf{4 4}\end{array}$

2.17.1 Media, buffer and stock solutions used for Yeast Two-Hybrid screen 44

2.17.2 Generation of Gal4 DNA-BD/bait construct 45

2.17.3 Transformation of bait plasmid into yeast (small scale transformation) 45

2.17.4 Transformation of yeast cells with cDNA library (large scale transformation) 45

2.17.5 Isolation of plasmid DNA from yeast $\quad 46$

2.17.6 Identification of interacting cDNA clones 46

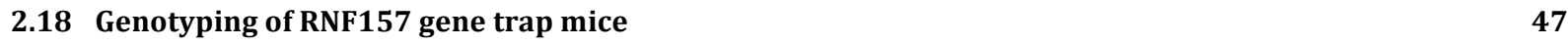

$\begin{array}{ll}\text { 2.18.1 Extraction of genomic DNA from mouse tails } & 47\end{array}$

$\begin{array}{lll}\text { 2.18.2 RNF157 Genotyping PCR } & 47\end{array}$

$\begin{array}{llr}2.19 & \text { Statistics } & 47\end{array}$

$\begin{array}{lll}3 & \text { Results } & 48\end{array}$

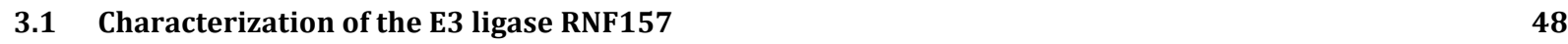

$\begin{array}{lll}3.1 .1 & \text { Cloning of rat RNF157 cDNA } & 48\end{array}$

3.1.2 Expression and localization of RNF157 during brain development 49 


\subsubsection{Subcellular localization of RNF157 in neurons}

3.2.1 Validation of RNF157 shRNA constructs $\quad 53$

3.2.2 RNF157 is a regulator of neuronal survival in cerebellar granule neurons $\quad 54$

3.2.2.1 Overexpression of RNF157 protects granule neurons from cell death under apoptotic stimuli 56

3.2.2.2 RNF157 promotes neuronal survival independently of the neuronal cell type 57

3.2.3 RNF157 promotes dendrite development and maintenance in cerebellar granule neurons $\quad 57$

3.2.3.1 Knockdown of RNF157 reduces dendritic but not axonal length in cerebellar granule neurons 58

3.2.3.2 Knockdown of RNF157 reduces dendrite complexity in cerebellar granule neurons 60

3.2.3.3 RNF157 is important for the maintenance of cerebellar granule neuron dendrites 60

3.2.3.4 Introduction of the Wallerian degeneration slow fusion protein (Wlds) has no effect on the RNF157knockdown induced dendritic phenotype

3.2.3.5 RNF157 significantly increases dendritic but not axonal length in an E3 ligase activity-independent manner in cerebellar granule neurons $\quad 63$

3.2.3.6 Overexpression of RNF157 influences dendritic arborization 64

3.2.3.7 RNF157 promotes dendrite development independently of the neuronal cell type 64

3.3 Identification of RNF157 interacting proteins in the mammalian brain 66

3.3.1 Yeast Two-Hybrid screen $\quad 66$

3.4 Fe65, a novel interactor of RNF157, acts in concert with RNF157 in the control of neuronal survival 67

$\begin{array}{lll}3.4 .1 & \text { Fe65 is a novel interactor of RNF157 } & 67\end{array}$

3.4.1.1 Mapping the specific binding regions of RNF157 and Fe65 68

3.4.2 Expression and localization of Fe65 during brain development 69

$\begin{array}{lll}\text { 3.4.3 Ubiquitination of Fe65 in cerebellar granule neurons } & 71\end{array}$

$\begin{array}{lll}3.4 .4 & \text { Fe65 is ubiquitinated by RNF157 } & 72\end{array}$

3.4.5 Knockdown of Fe65 has no effect on dendrite growth in cerebellar granule neurons 73

3.4.6 Expression of Fe65 induces apoptosis in cerebellar granule neurons $\quad 74$

3.4.7 Knockdown of Fe65 reduces starvation-induced apoptosis in cerebellar granule neurons 78

3.4.8 RNF157 knockdown-induced increase in neuronal apoptosis can be alleviated by simultaneous knockdown $\begin{array}{ll}\text { of the death receptor } 6 \text { (DR6) } & 79\end{array}$

3.4.9 Fe65 acts downstream of RNF157 in a linear pathway in the control of neuronal survival 80

3.5 Characterization of a mouse model lacking RNF157 function 8

3.5.1 Generation of RNF157 gene trap mice $\quad 81$

3.5.2 Analysis of RNF157 transgenic animals 82

$\begin{array}{lll}\text { 3.5.2.1 Histologic analysis of RNF157 heterozygous mice } & 83\end{array}$

\section{Discussion $\quad 85$}

4.1 RNF157 is a brain-dominant protein that is expressed throughout development 85

$\begin{array}{lll}4.2 & \text { RNF157 promotes neuronal survival in vitro } & 87\end{array}$

4.3 RNF157 is a novel regulator of dendrite growth independently of its E3 ligase activity 89

4.3.1 Wlds has no effect on the RNF157-knockdown induced dendritic phenotype 91

4.4 Fe65, a novel interactor of RNF157, acts in concert with RNF157 in the control of neuronal survival 92

4.4.1 Expression and localization of Fe65 during CNS development 93

4.4.2 Fe65 is ubiquitinated by the E3 ligase RNF157 94

4.4.3 RNF157 and Fe65 act in a linear pathway to control neuronal survival 95 
4.4.3.1 RNF157 and death receptor 6 (DR6) act in a linear pathway to control neuronal survival

4.5 Generation and characterization of a mouse model lacking RNF157 function

4.6 Conclusion

5 Appendix

101

5.1 Alignment of mouse, human and predicted rat cDNA sequence

6 References 103

Curriculum Vitae 


\section{Acknowledgments}

First, I want to thank my supervisor Dr. Judith Stegmüller, who supported me throughout my thesis with her patience and knowledge, while allowing me the room to work on my own. I am sure it would have not been possible without her help. I am very grateful to her for training me in experimental as well as presentation/writing skills and for the proofreading of the study.

Additional thanks go to Prof. Dr. Tomas Pieler and Prof. Dr. Anastassia Stoykova for being members of my thesis committee, the support throughout my $\mathrm{PhD}$ and the fruitful discussions about my project, which were always a great help for me.

I would like to show my gratitude to Nicola Schwedhelm-Domeyer for her help during the project and especially for her personal support during the four years of my PhD. It was a great time with you!

Additional thanks go to Shih-Ju Lee who is involved in the project and helps with her work to understand the role of RNF157 in neuronal survival. Furthermore, I want to thank Anna Holubowska who contributed valuable results to this work. It was a pleasure to work with you.

Many thanks to all the other lab members for the great working atmosphere and cooperation: Dr. Madhuvanthi Kannan and David Brockelt. Special thanks goes to Chaitali Mukherjee and Siv Vingill for proofreading of my thesis and Mayur Vadhvani for answering all my questions!

Moreover, I want to gratitude my close friends Elina, Thomas, Evelyn, Annette, Maria and Nils for their support throughout the time. I wish you all the best. Thank you!

Most importantly, I am grateful to my boyfriend Sofoklis. Thank you so much for all your love, support and understanding. You mean the world to me!

Finally, I want to express my deepest gratitude to my parents Wolfgang and Monika Matz for their unconditional support. I want to thank you from the bottom of my heart for all the motivation, encouragement and advice. You are all real troopers! 


\section{Summary}

Apoptosis or programmed cell death is a crucial event during neuronal development to reduce excessive neurons. In addition, neuronal apoptosis is the cause of many neurodegenerative diseases including Alzheimer's disease or Parkinson's disease. Emerging evidence implicates the ubiquitin-proteasome system (UPS) in apoptosis and neurodegeneration. Ubiquitination of intracellular proteins by E3 ubiquitin ligases brings about degradation by the proteasome or serves as important regulatory modification. RING (ㄹeally Interesting New Gene) finger proteins represent the largest group of E3 ligases. Although more then 600 different E3 ligases are encoded by the human genome, the role of only a few RING E3 ligases has been characterized. Among those, the E3 ligase Mahogunin RING finger-1 (Mgrn1) has been implicated in progressive spongiform neurodegeneration. Interestingly, RNF157 is a homologue of Mgrn1 and shares a high level of similarity with Mgrn1. My work revealed that in contrast to Mgrn1, RNF157 displays a brain-dominant expression. Furthermore, I found that RNF157 is a cytoplasmic protein that is excluded from the nucleus in neurons. Morphological analyses uncovered a dual role for RNF157 in neurons: RNF157 promotes dendrite morphogenesis and it is a crucial regulator of neuronal survival. Interestingly, RNF157's E3 ligase activity is required only for the latter. To determine RNF157 substrates, I carried out an interaction screen and identified Fe65 as a novel interactor of RNF157. Subsequently, after validation of the RNF157/Fe65 interaction in heterologous cells and in vivo, my work has uncovered that Fe65 is ubiquitinated by RNF157 in a non-proteolytic manner. In addition, functional analyses showed that Fe65 is a pro-apoptotic protein in neurons, which operates in the nucleus to initiate apoptosis. Consistent with these results, epistasis analysis revealed that Fe65 acts downstream of RNF157 in the control of neuronal survival. In conclusion, my study uncovers a novel RNF157-Fe65 pathway that regulates neuronal survival in vitro. In addition, my work was accompanied by the generation of an RNF157 knockout mouse model, which demonstrates the importance of RNF157 during embryonic development. Thus, my research significantly contributed to the understanding of RING E3 ligases in neuronal survival and provides novel insight into a pathway that might be implicated in neurodegeneration. 


\section{Abbreviations}

\section{Abbreviation}

AD

Ago1

AICD

ALS

Apaf-1

APC

APP

$A B$

ATM

ATR

BAF

BDNF

BMP

CAM

CaMK

CDT

CGN

CHIP

CIP

DCC

DD

DISC

DIV

DNA-AD

DNA-BD

DR

DSB

Dscam

DTT

\section{Meaning}

Alzheimer's disease

RNase Argonaute 1

APP intracellular domain

Amyotrophic lateral sclerosis

Apoptotic protease-activating factor-1

Anaphase-Promoting Complex

amyloid precursor protein

amyloid beta

ataxia-telangiectasia-mutated

ataxia-telangiectasia Rad-related

$\mathrm{Brg} / \mathrm{Brm}$-associated factor

brain-derived neurotrophic factor

bone morphogenic protein

cell adhesion molecules

$\mathrm{Ca}^{2+} /$ calmodulin-dependent protein kinase

Cerebellar Development Transcriptome

cerebellar granule neuron

carboxyl terminus of Hsp70-interacting protein

calf intestinal phosphatase

depleted in colorectal carcinoma

death domain

death-inducing signaling complex

Day in vitro

DNA-activation domain

DNA-binding domain

death receptor

DNA double strand-breaks

Down syndrome-related cell adhesion molecule

Dithiothreitol 


\begin{tabular}{|c|c|}
\hline $\mathrm{E}$ & embryonal \\
\hline EGL & external granule layer \\
\hline endoG & endonuclease G \\
\hline ES & embryonic stem \\
\hline GAP & GTPase activating proteins \\
\hline GEF & guanine nucleotide exchange factor \\
\hline GFAP & Glial fibrillary acidic protein \\
\hline GPI & glycosylphospatidylinositol \\
\hline GSK3 & glycogen synthase 3 \\
\hline HDM2 & human double minute 2 \\
\hline HECT & homologous to E6AP Carboxyl Terminus \\
\hline Het & heterozygous \\
\hline Hom & homozygous \\
\hline HRP & horseradish peroxidase \\
\hline IAP & inhibitor of apoptosis protein \\
\hline IGL & internal granular layer \\
\hline IP & progenitor cell \\
\hline JAM C & junction adhesive molecule $\mathrm{C}$ \\
\hline JNK & c-Jun N-terminal kinase \\
\hline $\mathrm{kb}$ & kilo-base pair \\
\hline $\mathrm{kDa}$ & kilodalton \\
\hline LRP1 & lipoprotein receptor-related protein \\
\hline MAP & microtubule-associated protein \\
\hline MDD & major depressive disorder \\
\hline $\mathrm{mg}$ & milligram \\
\hline Mgrn1 & Mahogunin RING finger-1 \\
\hline $\mathrm{mM}$ & millimolar \\
\hline NEDD & $\begin{array}{l}\text { neuronal precursor cell expressed } \\
\text { developmentally down regulated }\end{array}$ \\
\hline NF & Nuclear fraction \\
\hline ng & nanogram \\
\hline NT & neurotrophin \\
\hline OGD & oxygen and glucose deprivation \\
\hline$P$ & postnatal day \\
\hline
\end{tabular}




\begin{tabular}{|c|c|}
\hline PAR & partitioning defective \\
\hline PCD & programmed cell death \\
\hline PD & Parkinson's disease \\
\hline PFA & paraformaldehyde \\
\hline PHD & plant homeobox domain \\
\hline PI3K & phosphatidylinositol 3-kinase \\
\hline PNS & postnuclear supernatant \\
\hline PSD-95 & postsynaptic density protein 95 \\
\hline РТB & phosphotyrosine binding \\
\hline RGC & radial glial cell \\
\hline RING & really interesting new gene \\
\hline RNF & RING finger protein \\
\hline Robo & roundabout \\
\hline ROS & reactive oxygen species \\
\hline rpm & rounds per minute \\
\hline $\mathrm{RT}$ & room temperature \\
\hline SDS & sodium dodecyl sulfate \\
\hline SEM & standard error of the mean \\
\hline Shh & sonic hedgehong \\
\hline Siah & seven in Absentia homolog \\
\hline Smac/Diabolo & $\begin{array}{l}\text { second mitochondria-derived activator } \\
\text { caspases/direct IAP binding protein of low pI }\end{array}$ \\
\hline TGF $\alpha$ & transforming growth factor \\
\hline TIP & microtubule plus-end tracking protein \\
\hline TNF & tumor necrosis factor \\
\hline TNFR1 & TNF-receptor 1 \\
\hline TujI & class III beta-tubulin \\
\hline UAS & upstream activating sequence \\
\hline UPS & ubiquitin-proteasome system \\
\hline$\mu g$ & microgram \\
\hline$\mu \mathrm{m}$ & micrometer \\
\hline WT & wild type \\
\hline
\end{tabular}




\section{Abbreviations for amino acids:}

$\begin{array}{lcc}\text { Amino acid } & \text { 3 letter code } & \text { 1 letter code } \\ \text { Alanine } & \text { Ala } & \text { A } \\ \text { Arginine } & \text { Arg } & \text { R } \\ \text { Asparagine } & \text { Asn } & \text { N } \\ \text { Aspartic acid } & \text { Asp } & \text { D } \\ \text { Cysteine } & \text { Cys } & \text { C } \\ \text { Glutamic acid } & \text { Glu } & \text { E } \\ \text { Glutamine } & \text { Gln } & \text { Q } \\ \text { Glycine } & \text { Gly } & \text { G } \\ \text { Histidine } & \text { His } & \text { H } \\ \text { Isoleucine } & \text { Ile } & \text { I } \\ \text { Leucine } & \text { Leu } & \text { L } \\ \text { Lysine } & \text { Lys } & \text { K } \\ \text { Methionine } & \text { Met } & \text { M } \\ \text { Phenylalanine } & \text { Phe } & \text { F } \\ \text { Proline } & \text { Pro } & \text { P } \\ \text { Serine } & \text { Ser } & \text { S } \\ \text { Threonine } & \text { Thr } & \text { T } \\ \text { Tryptophan } & \text { Trp } & \text { W } \\ \text { Tyrosine } & \text { Tyr } & \text { Y } \\ \text { Valine } & \text { Val } & \text { V } \\ \text { Any possible amino acid } & & \text { X }\end{array}$

Nucleotide

Adenosine

Cytosine

Guanine

Thymine
1 letter code

A
C
G
T




\section{Introduction}

\subsection{Neuronal development in the mammalian brain}

The human brain contains more than $10^{11}$ neurons that ensure intricate wiring and connectivity. Neurons exhibit a characteristic polarized morphology and thus harbor typically one axon and multiple dendrites. In humans, the length of an axon ranges from 1 $\mathrm{mm}$ to more than $1 \mathrm{~m}$. Neurons make connections via synapses to thousand other neurons and form a complex network that is responsible for transmission of information in the brain. In a directional flow, synaptic inputs from surrounding neurons are integrated by dendrites, provoking the generation of an action potential at the soma, which is propagated along the axon to other target cells. In addition to neurons, glial cells provide a supporting framework to maintain neuronal functionality and outnumber neurons 10-fold in vertebrates. During neuronal development, neurons undergo radical changes in their morphology. Immature neurons migrate long distances to their target location in the developing brain. This is followed by directed outgrowth of axonal and dendritic processes to make synaptic contacts with other cells, generating a highly organized network of connections between the different parts of the brain. Based on electrical inputs between the individual neurons, the connections are refined and adjusted throughout adulthood. Neuronal development can be subdivided into different key phases, which will be introduced in the following chapters.

\subsubsection{Neuronal induction}

Early in vertebrate development, the embryo is separated into three different layers - the endoderm, the mesoderm and the ectoderm. The latter is giving rise to the cells that are destined to become neurons of the central and peripheral nervous system (De Robertis \& Sasai, 1996). In the first phase of neuronal development, referred to as neuronal induction, cells in the ectoderm are determined to become neuronal precursors. This is evoked by diffusible molecules, the so-called neuronal inducers including Noggin, Chordin, Follistatin or Cerberus, which are secreted from dorsal cells of the mesoderm, the so-called organizer (Bouwmeester et al, 1996; Hemmati-Brivanlou et al, 1994; Lamb et al, 1993; Piccolo et al, 1999; Piccolo et al, 1996; Sasal et al, 1995; Zimmerman et al, 1996). These 
proteins are antagonists of bone morphogenetic proteins (BMPs) that bind to ectodermal cells to induce the transcription of epidermal genes. Inhibition of the BMP signaling pathway in turn induces the transcription of proneural genes followed by morphogenic changes of the ectodermal cells (Chang \& Hemmati-Brivanlou, 1998). These cells elongate into neuroepithelial cells along the whole axis of the embryo to form the neural tube, which ultimately develops into the brain and spinal cord. Depending on their localization along the anteroposterior axis of the neural tube, neuronal precursors are exposed to a gradient of different opposing morphogens including BMP, sonic hedgehong (Shh) and Wnt signals. This leads to the expression of a specific blend of transcription factors, thereby determining the developmental fate of the neuronal precursor (Dessaud et al, 2008; Falk \& Sommer, 2009; Lee \& Jessell, 1999; Lupo et al, 2006). At tissue level, the neuronal tube becomes invaginated and the immature cells line the inner lumen of the ventricular zone. Simultaneously, neuroepithelial cells begin to divide and initiate the process of neuronal proliferation.

\subsubsection{Neuronal proliferation}

As neurogenesis proceeds, neuroepithelial cells in the anterior-dorsal part of the neural tube divide extensively to produce large amounts of cells, which give rise to the cerebral cortex of the mammalian brain (Stern, 2001). Hence during division, cells undergo a series of morphological changes, beginning with the extension of a process that spans the whole thickness of the ventricular zone. In the S phase, the nucleus travels to the upper region of the ventricular zone and returns to the apical layer during the G2 phase. Finally, the cell retracts the process, divides and the two doughter cells re-enter the cycle (McConnell, 1995).

In the developing neocortex, which later forms most of the cerebral cortex in humans, three main types of cells can be identified: radial glial cells (RGCs), intermediate progenitors and neuroepithelial cells. Initially, neuroepithelial cells at the ventricular zone proliferate by symmetric division to generate RCGs. These RCGs in turn expand by symmetric division that doubles the amount of cells after each mitotic cycle. Then, RCGs start to divide asymmetrically to produce another RCG and an intermediate progenitor (IP) or a neuron (Fishell \& Kriegstein, 2003; Noctor et al, 2001). RCGs however retain the ability to proliferate by symmetric division, whereas an IP divides once by symmetric division to produce two neurons at the subventricular zone (Noctor et al, 2004; Qian et al, 
2000). These newborn neurons travel remarkable distances to reach their final destination in the developing cerebral cortex.

\subsubsection{Neuronal migration}

Neurons in the developing cortex migrate along RGCs from the ventricular and the subventricular zone towards the cortical plate. After cell cycle exit, neurons become polarized and extend a leading process that senses the surrounding environment to determine the direction of movement. Then, the nucleus translocates along the leading process in a saltatory pattern, referred to as nucleokinesis. Finally, elimination of the trailing process leads to the net movement of the cell (Ayala et al, 2007). Neurons undergoing this locomotor process use radial glial fibers as a guide to reach the cortical plate, thus the radial glial-neuron interaction is of crucial importance (Hatten, 1999; Rakic, 1971; Rakic, 1972; Sidman \& Rakic, 1973). This interaction is mediated by various membrane-bound cell adhesion molecules that include astrotactin, neuregulin or different members of the family of integrins (Adams et al, 2002; Anton et al, 1997; Edmondson et al, 1988; Fishell \& Hatten, 1991; Stitt \& Hatten, 1990).

The beginning of cortical neuron migration is initiated by the release of chemoattractants including brain-derived neurotrophic factor (BDNF), neurotrophin (NT) 4, transforming growth factor (TGF) $\alpha$ or neurotransmitters (GABA and glutamate), while the direction of migration is guided by secreted molecules from the slit, netrin and semaphorin families. At the cortical plate, Cajal-Retzius cells secrete reelin, whose binding to its receptor triggers the phosphorylation of Dab1, which induces neurons to settle at appropriate layers in the cortex (Howell et al, 1999; Howell et al, 2000). Later born neurons migrate past their predecessors to form layers in an inside-out manner.

\subsubsection{Neuronal polarization: Establishment of axon-dendrite polarity}

The proper polarization of neurons is of major importance for the assembly of functional networks in the mammalian brain. The specification of a neuron into functionally distinct domains, i.e. the formation of a single axon and multiple dendrites, is a prerequisite for the integration and transmission of information in the brain. Both, the complex interaction of extracellular cues and intrinsic pathways are required for proper neuronal polarization. 


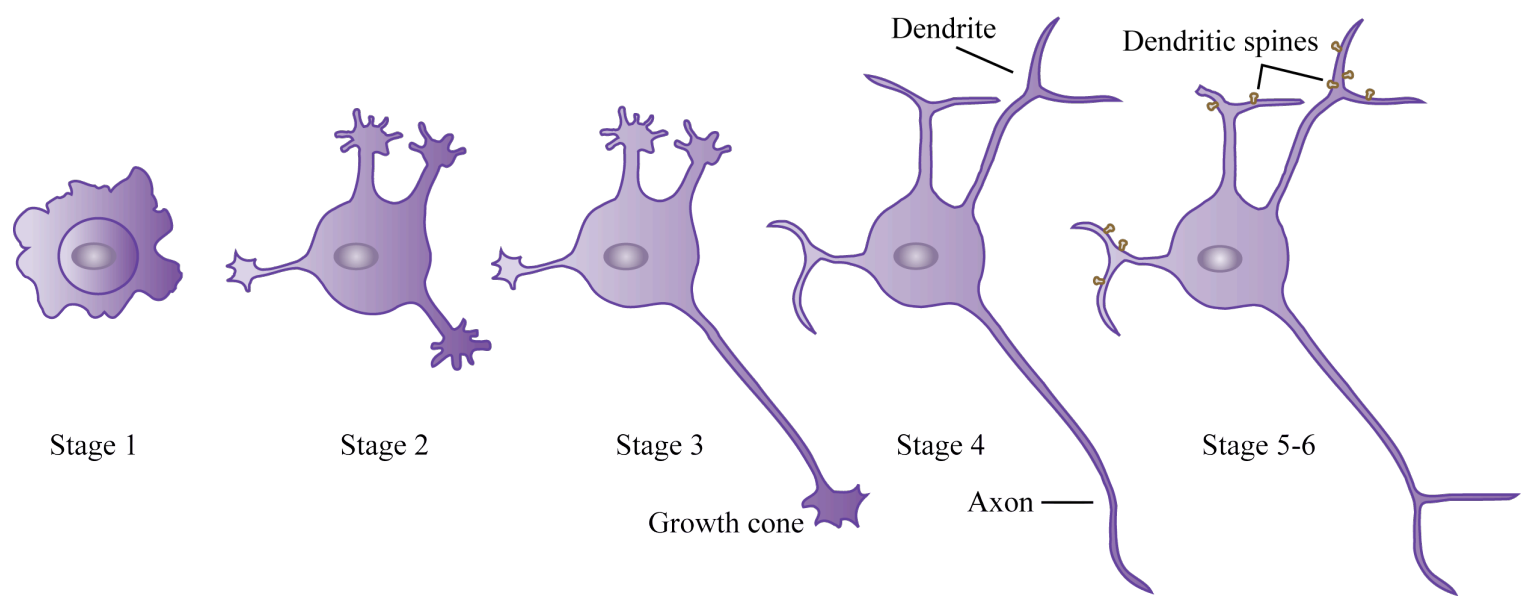

Figure 1. Neuronal polarization of hippocampal neurons in vitro. Shortly after plating, embryonic hippocampal neurons form a lamellipodium (stage 1) and subsequently transform into mutipolar cells with growth cones at their tips (stage 2). Then, one neurite starts to grow at a rapid rate and breaks the initial symmetric morphology to establish polarity (stage 3). This process will later become the axon, while the remaining neurites elongate and acquire the characteristics of dendrites (stage 4). During functional maturation, dendritic spines and synapses are formed to establish neuronal networks (stage 5-6) (modified from Arimura and Kaibuchi, 2007).

In 1994, Craig and Banker cultured hippocampal neurons to establish a model system to study neuronal polarization in vitro (Craig \& Banker, 1994). The morphological changes of hippocampal neurons during maturation follow a well described process (Dotti et al, 1988), (Figure 1). Shortly after plating, neurons extend small protrusions (stage 1) with growth cones at their tips that develop into equal immature neurites (stage 2). Then, one neurite breaks the cellular symmetry by rapid outgrowth into a long neurite, thereby establishing neuronal polarity (stage 3). This longer neurite becomes the axon, while the remaining shorter neurites acquire the characteristics of dendrites within the next days in culture (stage 4). The axon and dendrites continue functional maturation. After approximately seven days in culture (DIV), dendritic spines and axon terminals form synaptic contacts, thereby establishing a neuronal network during functional maturation (stage 5-6). Rearrangement of the cytoskeleton and microtubules are crucial to the formation of the axon (Tahirovic \& Bradke, 2009). To break the initial neuronal symmetry, intracellular signaling pathways trigger enhanced actin turnover at the growth cone of the future axon (Bradke \& Dotti, 1999; Witte \& Bradke, 2008).

In contrast, the remaining neurites possess a rigid actin cytoskeleton that is nonpermissive for microtubule protrusions, thus inhibiting enhanced neurite elongation (Bradke \& Dotti, 1999; Forscher \& Smith, 1988). Further, pharmacological actin destabilization induces the formation of multiple axons (Witte \& Bradke, 2008). This indicates the importance of cytoskeletal dynamics in axon specification and neuronal 
polarization. An array of proteins embedded in different signaling pathways converge at the level of cytoskeletal regulation. Among those, Rho-GTPases, phosphatidylinositol 3kinase (PI3K), enabled/vasodilator stimulated phosphoprotein (Ena/VASP), cofilin, SAD kinases and partitioning defective (PAR) complex proteins have been identified as key regulators of neuronal polarity (Barnes \& Polleux, 2009; Tahirovic \& Bradke, 2009).

\subsubsection{Axonal growth and guidance}

The correct wiring of the developing nervous system depends on the ability of axons and dendrites to make contacts with their appropriate synaptic partners. During axonal pathfinding, the growth cone of the axon detects guidance cues secreted by cells of the environment. These molecules attract or repel the growth cone either at close range or over long distances (Tessier-Lavigne \& Goodman, 1996). Researchers have identified several families of guidance cues and receptors, including semaphorins and their plexin (Plex)-and neuropilin receptors (Pasterkamp \& Kolodkin, 2003), netrins and their depleted in colorectal carcinoma (DCC)-and UNC5 receptors (Kennedy, 2000), slits and their roundabout (Robo) receptors (Brose \& Tessier-Lavigne, 2000), and ephrins and their Eph receptors (Kullander \& Klein, 2002). Interestingly, the composition of receptors present at the growth cone determines the direction of axonal growth rather than a given guidance cue (Garbe \& Bashaw, 2004; Huber et al, 2003). Other guidance cues including secreted proteins of the Wingless/Wnt (Lyuksyutova et al, 2003; Yoshikawa et al, 2003), sonic hedgehog (Shh) (Charron et al, 2003) and Dpp/BMP/TGF-ß (Butler \& Dodd, 2003) families contribute to axon guidance. Furthermore, neurotrophins, known regulators of neuronal growth and survival, have been shown to guide axons in vitro (Gundersen \& Barrett, 1979; Letourneau, 1978; Song et al, 1997). Directed axonal elongation is also supported by the interaction of specific environmental cues with cell adhesion molecules (CAMs), including integrins, cadherins and the immunoglobin superfamily. They act as contact-dependent attractive/repulsive cues and regulate selective fasciculation/defasiculation of axons (Tessier-Lavigne \& Goodman, 1996).

The interaction of guidance cues with their receptors leads to the activation of signaling cascades. While it is widely accepted that these signals converge at the cytoskeletal level to control growth cone dynamics, the underlying mechanisms remain incompletely understood. A crucial event however is the regulation of the activity of RhoGTPases. This protein family, including the well-studied members Rac, Cdc42 and RhoA, 
catalyzes the hydrolysis of bound GTP to GDP, thereby switching from active to inactive state (Hall, 1998; Luo, 2000; Luo, 2002). Slits, netrins, ephrins and semaphorins differentially regulate the activity of these small GTPases either by direct interaction (Vikis et al, 2002; Vikis et al, 2000) or via RhoGEFs (guanine nucleotide exchange factors) and RhoGAPs (GTPase activating proteins). This leads to remodeling of the cytoskeleton at the growth cone by regulating actin polymerization and depolymerization (Welch \& Mullins, 2002), actin/myosin contractility (Hirose et al, 1998) and microtubule reorganization (Palazzo et al, 2001). Besides these extracellular mechanisms, cell-intrinsic signaling pathways have emerged as crucial regulators of axonal morphogenesis. The ubiquitinproteasome system and several transcription factors have been implicated in the regulation of important aspects of axon outgrowth and pathfinding (Moore \& Goldberg, 2011; Polleux et al, 2007).

\subsubsection{Cellular and molecular mechanisms of dendrite growth and synapse formation}

The morphology of different neuronal cell types is also characterized by a cell-type specific dendritic morphology (Ramón y Cajal, 1911), (Figure 2). Dendritic arborization is regulated in a complex and multi-step process that is controlled at each stage both by extrinsic cues and cell-intrinsic programs (Jan \& Jan, 2003; McAllister, 2000; Urbanska et al, 2008; Wong \& Ghosh, 2002).

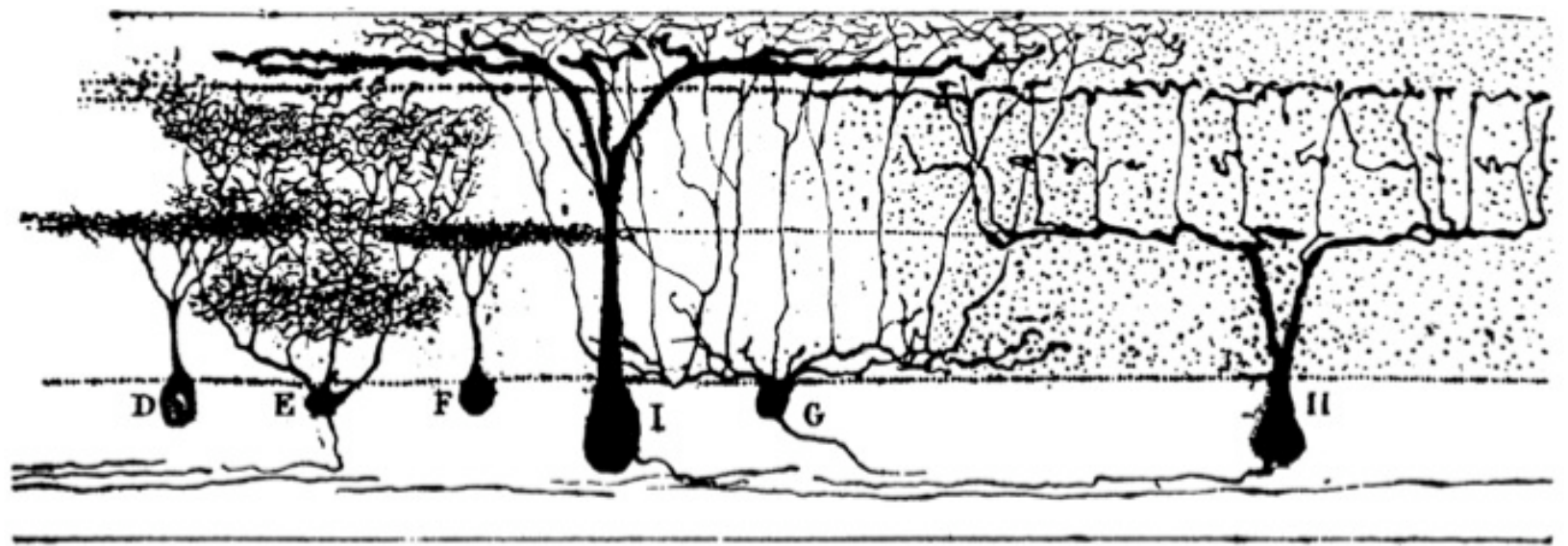

Figure 2. Diverse dendritic branching pattern of ganglion cells in the retina of the spotted lizard (Ramón y Cajal 1911).

Dendritic development can be divided into several essential steps including neurite initiation, outgrowth and guidance, branching and synapse formation and stabilization of the dendritic arbor. First, after neuronal polarization and axonal elongation, the remaining 
neurites obtain dendrite characteristics in length, diameter, growth rate and molecular composition. Second, after extensive elongation in a defined direction, dendrites increase in diameter and start branching either via bifurcation of the growth cone-like tips or via interstitial sprouting of a new branch from an already existing one. This phase is highly dynamic since the addition and elimination of new branches occur at a high rate, while only a subset of branches become stabilized. Third, dendrites generate dendritic spines that are the major sites of excitatory synapses. Fourth, after acquiring its final shape, the dendritic arbor becomes stabilized, which is accompanied by a decrease in structural plasticity (Craig \& Banker, 1994; Jan \& Jan, 2003; Scott \& Luo, 2001). The proper development of dendritic arborization and hence the respective dendritic branching pattern defines the amount of synaptic inputs that a neuron receives. Thus, defects in dendrite growth and patterning can lead to severe dysfunction of the central nervous system (CNS).

\subsubsection{Extracellular signals in the control of dendrite growth and branching}

Dendritogenesis is regulated by a combination of diffusible molecules, cell-cell interactions and importantly neuronal activity. These diffusible cues include proteins of the neurotrophin family (NGF, BDNF, NT3 and NT4), BMPs, cpg15, reelin, semaphorins and slits (Jan \& Jan, 2003; McAllister, 2000; Parrish et al, 2006; Wong \& Ghosh, 2002). Neurotrophins have been shown to regulate dendrite growth in the developing neocortex, thereby conferring a specific shape to the dendrites of the different cortical layers (Baker et al, 1998; McAllister et al, 1995). Furthermore, the BMP family member OP-1 /BMP7 also modulates dendrite growth and arborization in rat sympathetic and cultured cortical neurons, respectively (Guo et al, 1998; Le Roux et al, 1999). Another regulator of dendrite growth is the synaptic activity-regulated glycosylphospatidylinositol (GPI)-linked protein cpg15 that exclusively enhances dendrite growth in projection neurons of the optic tectum in Xenopus (Nedivi et al, 1998). Also, the well-established effect of reelin on dendrite growth and branching of hippocampal neurons is mediated by mTor, which is in contrast to the PI3K and Akt-dependent organization of the cortical plate (Jossin \& Goffinet, 2007). Semaphorin 3A exerts its chemoattractant function on apical cortical dendrite growth through the neuropilin-1 receptor and selective distribution of the adenylat cylase in apical dendrites (Polleux et al, 1998; Polleux et al, 2000). In another study, Whitford and colleagues showed that the slit/Robo interaction regulates dendrite growth and branching in cortical neurons (Whitford et al, 2002). 
In addition to extracellular molecules, cell membrane proteins including Delta and Notch (Redmond et al, 2000; Sestan et al, 1999), contactin (Berglund et al, 1999), cell adhesion molecule L1 (Demyanenko et al, 1999), ephrinB and EphB (Hoogenraad et al, 2005), N-Cadherins (Yu \& Malenka, 2003) and seven-pass transmembrane cadherins (Flamingo, Celsr2 and Celsr3) (Gao et al, 2000) have been implicated in controlling dendrite growth, branching and tiling, a process that regulates the spacing of the dendritic arbor (Parrish et al, 2007). Down syndrome-related cell adhesion molecule (Dscam) is known to mediate dendrite self-avoidance, which prevents dendrites from making contact with one another, mostly within the same neuron. Alternative splicing of Dscam results in an enormous amount of different isoforms; expression of the same isoform by sister dendrites triggers mutual repulsion in several types of Drosophila neurons (Hughes et al, 2007; Zhu et al, 2006). Interestingly, a similar behavior was reported recently by Smith and colleagues for the diffusible guidance cue UNC6 (Netrin) that forms a complex with UNC40 and UNC5 in dendro-dendritic contacts, resulting in contact-dependent repulsion in $C$. elegans (Smith et al, 2012). Additionally, since glial cells display a crucial supporting framework for proper neuronal functionality, neuron-glial interactions are important for dendrite growth and arborization in both peripheral and central nervous system (Tropea et al, 1988).

Since dendritic differentiation is accompanied by synapse formation, neuronal activity is another important aspect during dendrite development. While blocking or decreasing afferent activity provokes stunted dendritic arbors, exposure to an enriched environment can increase dendrite growth and branching in several brain regions of rat pups (Holloway, 1966; Volkmar \& Greenough, 1972). In addition, neuronal activity affects the amount of dendritic filopodia and spines in many regions of the brain (McAllister, 2000). The activity-dependent effect on dendrite arborization is mediated by elevated intracellular calcium levels that activate $\mathrm{Ca}^{2+}$-dependent downstream signaling pathways (Wong \& Ghosh, 2002).

\subsubsection{Intracellular mechanisms regulating dendrite growth and branching}

Growing evidence suggests that cell-intrinsic mechanisms including regulators of the cytoskeleton and several transcription factors play a crucial role during dendrite development. Since microfilaments and microtubules represent the basic framework of dendrites, changes in growth or branching result from polymerization dynamics of the 
actin and microtubule cytoskeleton. Microtubule-associated proteins (MAPs) including MAP1a, MAP1B and MAP2 have been reported to play an important role in proper dendritic arbor morphology by stabilizing-and cross-linking microtubules to the actin cytoskeleton (Georges et al, 2008). For example, depletion of MAP2 in mice causes a reduction in dendrite length in hippocampal neurons (Harada et al, 2002). Furthermore, the microtubule-associated motor proteins Lis-1 and dynein, members of the microtubule plus-end tracking protein (+TIP) family, regulate dendrite arborization of Drosophila mushroom-body neurons (Liu et al, 2000).

In addition to cytoskeletal regulators, transcriptional regulation contributes to the refinement of dendrite arbor formation (Urbanska et al, 2008). Wu and colleagues demonstrated the importance of transcription in activity-regulated dendrite growth in BAF53b knockout mice. BAF53b, which is exclusively found in neurons, is a subunit of the chromatin-remodeling Brg/Brm-associated factor (BAF) complex (Olave et al, 2002), which regulates the accessibility of transcription factors to interact with DNA by ATP-dependent chromatin remodeling. Together with the $\mathrm{Ca}^{2+}$-dependent transcriptional coactivator CREST, nBAF regulates the transcription of genes involved in dendrite growth. Interestingly, BAF53b knockout mice were impaired in activity-induced dendrite outgrowth, which was similar to the defects in CREST knockout mice (Aizawa et al, 2004). $\mathrm{Wu}$ and colleagues found that BAF53b is required for the recruitment of the nBAF/CREST complex to the promoter region of dendrite growth regulating genes, thereby controlling dendrite morphogenesis (Wu et al, 2007).

Another important regulator of dendrite growth is the brain-enriched proneural bHLH transcription factor NeuroD. Gaudilliere and colleagues showed that RNAi-mediated knockdown of NeuroD dramatically impairs dendrite development in vitro. Furthermore, they found that NeuroD is activated through phosphorylation at serine 336 in an activitydependent manner by the $\mathrm{Ca}^{2+} /$ calmodulin-dependent protein kinase II (CaMKII), thus providing a link between neuronal activity and the regulation of dendrite morphogenesis (Gaudilliere et al, 2004). In addition, neurogenin2, another bHLH transcription factor, regulates pyramidal neuron morphology in vivo by specification of the leading edge polarity during neuronal migration (Hand et al, 2005). This further sheds light onto the important function of proneural bHLH transcription factors during dendrite morphogenesis. 
Cux1, a member of the homeobox family of transcription factors, regulates dendrite complexity in cultured cortical pyramidal neurons. While overexpression of Cux1, but not Cux2, significantly decreases cortical dendrite length, knockdown of Cux1 by RNAi increases the complexity of cortical neurons. Cux1 has further been identified to act as a transcriptional repressor of the cyclin-dependent kinase $\mathrm{p}^{27}$, which is a known regulator of the small GTPase RhoA (Besson et al, 2008). Thus, Cux1 negatively regulates cortical dendrite growth through the downstream factors p27 and RhoA (Li et al, 2010).

Hence, identification of proteins involved in dendrite morphogenesis is an essential step towards understanding the different stages of dendrite development.

\subsection{Neuronal apoptosis during development and disease}

Programmed cell death (PCD) is an important physiological process during neuronal development that eliminates excessive neurons generated during mitosis. However, neuronal apoptosis is not restricted to the period of brain development, but can be a reoccurring phenomenon in the adult brain when injury or insult trigger neuronal cell death. The term apoptosis, referring to the naturally occurring loss of leaves (from the Greek words apo-away from and ptosis-fall), is used to describe characteristic manifestations during PCD (Kerr et al, 1972). Apoptosis is a highly conserved process in eukaryotes that activates a sequence of signaling cascades (Martin et al, 1988; Metzstein et al, 1998). Morphological manifestations of apoptosis include shrinkage and condensation (karyopyknosis) of the cell body, fragmentation (karyorrhexis) of the nucleus as a result of intranucleasomal DNA cleavage, nuclear membrane breakdown and plasma membrane blebbing, and the formation of apoptotic bodies, which are subsequently phagocytosed by neighboring cells (Compton, 1992; Kerr et al, 1972). In contrast to apoptosis, necrotic cell death is a non-regulated form of cell death, characterized by swellings of cell organelles and release of intracellular components into the intracellular space, thereby provoking an inflammatory response (Farber, 1994). Autophagy displays another mechanism of cell death that is evolutionarily conserved and involves degradation of cellular organelles by lysosomes (Levine \& Yuan, 2005). All three forms of cell death occur more or less frequently in neuronal development and neurodegeneration (Lossi \& Merighi, 2003; Yuan et al, 2003). 


\subsubsection{The apoptotic cell death machinery and the Bcl-2 family}

The death of a neuron can be induced by the absence of trophic factors or by the presence of extrinsic and intrinsic apoptotic stimuli including $\mathrm{Ca}^{2+}$, glutamate, reactive oxygen species (ROS) or excitotoxicity (Figure 3). These signals converge at the mitochondrial apoptotic machinery to induce the release of cytochrom c, provoking the activation of caspases and subsequent cleavage of key cellular components.

Cytochrom c is a soluble 13-kDa protein that is involved in electron transfer in the mitochondrial intermembrane space (Liu et al, 1996). The release of cytochrom c is regulated by Bcl-2 family proteins, which are critical regulators of cell death. They are divided in pro-apoptotic (Bax, Bak, etc.), anti-apoptotic (Bcl-2, Bcl- $\mathrm{x}_{\mathrm{L}}, \mathrm{Bcl}-\mathrm{w}$, etc.) and BH3only (Bid, Bad, Noxa, etc) proteins containing one or more Bcl-2 homology domains. In response to an apoptotic stimulus, activation of certain BH-3 only proteins causes the activation of the pro-apoptotic proteins Bax or Bak at the mitochondrial membrane (Hsu et al, 1997; Nechushtan et al, 2001; Wolter et al, 1997; Zha et al, 1996). Homo-dimerization of Bax and Bak leads to formation of a pore in the outer mitochondrial membrane, followed by alterations of the mitochondrial membrane potential and the release of pro-apoptotic proteins including cytochrom c, second mitochondria-derived activator of caspases/direct IAP binding protein of low pI (Smac/Diabolo), AIF, HtrA2 or endonuclease G (endoG) (Jiang \& Wang, 2000). The anti-apoptotic Bcl-2 proteins block cell death by preventing activation and homo-dimerization of Bax and Bak, thus maintaining the integrity of the mitochondrial membrane. Hence, the decision of a neuron to undergo cell death is dependent on the relative levels of pro-and anti-apoptotic proteins (Oltvai et al, 1993).

In a caspase-dependent mechanism, cytochrom c activates apoptotic proteaseactivating factor-1 (Apaf-1) and induces the oligomerization of Apaf-1/cytochrom-c to form the apoptosome (Zou et al, 1997). This complex recruits and activates procaspase-9 that is subsequently released into the cytoplasm to activate downstream caspases including caspase-3, caspase-6 or caspase-7 (Li et al, 1997; Saleh et al, 1999; Srinivasula et al, 1998; Zou et al, 1997; Zou et al, 1999). Substrates of these effector caspases are mediators and regulators of apoptosis, including DNA fragmentation factor $45 \mathrm{kDa}$ subunit (DFF45/ICAD), $75 \mathrm{kDa}$ subunit of respiratory complex I (NDUFS19, structural proteins (fodrin and gesolin) and cellular DNA repair proteins (PARP, DNA-dependent protein kinase (DNA-PK)). Cleavage of these proteins causes many of the characteristic morphological hallmarks of apoptosis (Degterev et al, 2003). Concomitant release of 
SMAC/Diabolo and HtrA2 specifically sequesters IAP proteins, which are negative regulators of intracellular caspase activity (Suzuki et al, 2001; Verhagen et al, 2000). Moreover, while SMAC/Diabolo leads to activation of caspase-9, HtrA2 initiates fragmentation of the DNA. Alternatively, apoptosis is induced by a caspase-independent mechanism through AIF and endoG (Benn \& Woolf, 2004). While AIF functions in the nucleus downstream of PARP1 to initiate DNA fragmentation and chromatin condensation, the sequence unspecific DNase endoG degrades nuclear DNA (Arnoult et al, 2003; Daugas et al, 2000; Li et al, 2001; Susin et al, 1999; Yu et al, 2002).

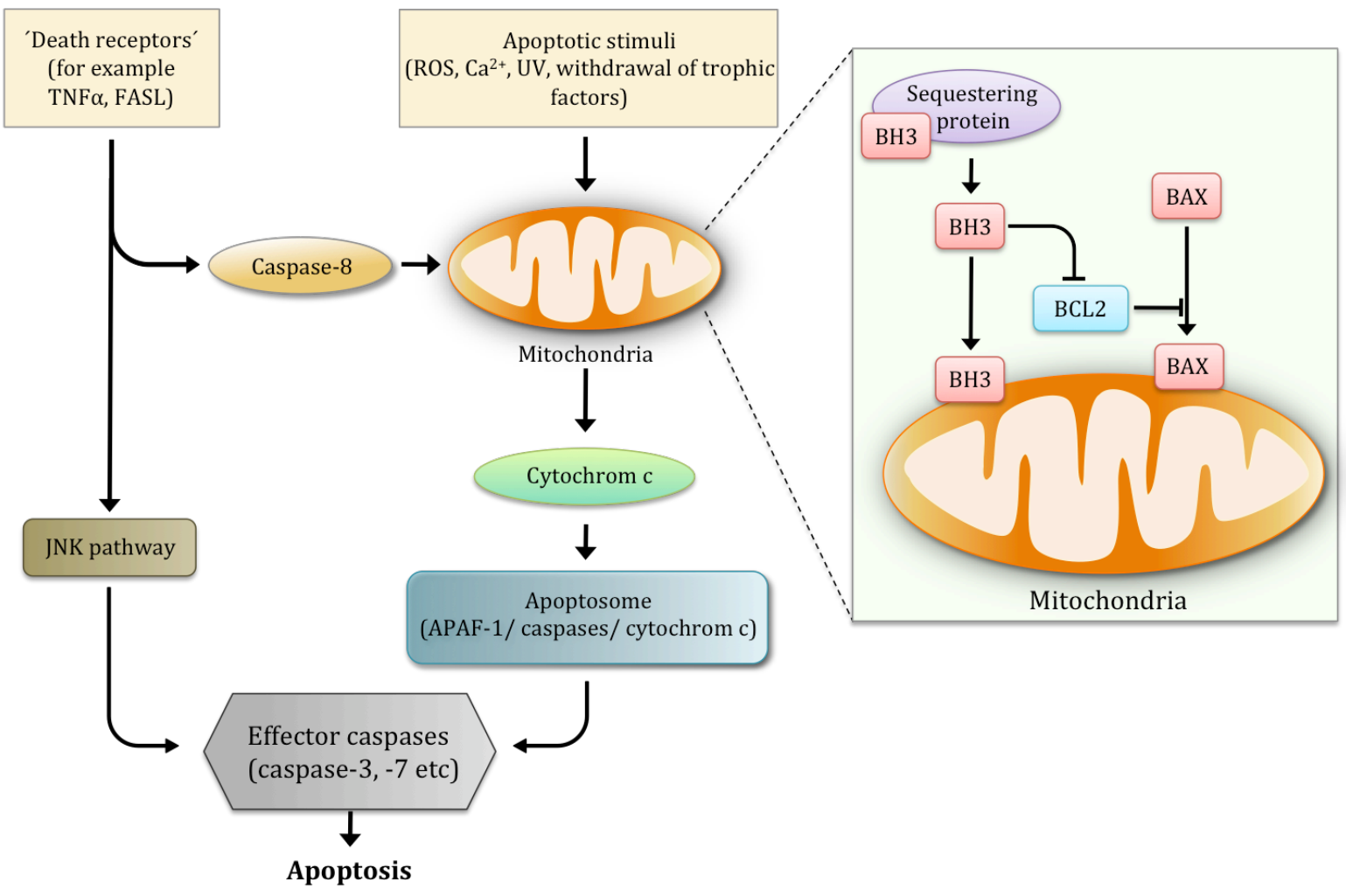

Figure 3: Overview of the apoptotic machinery in neurons. Neuronal apoptosis can be induced by the extrinsic activation of death receptors or by a plethora of different apoptotic stimuli inside and outside of the neuron. The death receptor pathway is activated in response to the binding of extracellular ligands, which can either directly lead to the activation of effector caspases through the apoptotic JNK (c-Jun-N-terminal kinase) signaling pathway or can converge with diverse apoptotic stimuli to initiate the apoptotic cell death machinery at the mitochondria. This involves the activation and homo-dimerization of pro-apoptotic BH3only proteins as Bax or Bak to form a mitochondrial membrane permeability pore, which results in alterations of the mitochondrial membrane integrity. These effects are accompanied by the subsequent inhibition of anti-apoptotic Bcl-2 family members. This is followed by the release of cytochrom c, formation of the apoptosome and subsequent activation of effector caspases to trigger cell death (modified from Benn and Woolf, 2004). 


\subsubsection{Programmed cell death during neuronal development}

During neuronal development neurons are produced in excess and approximately half of them are being weeded out by apoptosis before maturation of the CNS. Thus, neuronal apoptosis is believed to ensure the appropriate ratio of communicating neurons (Oppenheim, 1991). In 1949, Viktor Hamburger and Rita Levi-Montalcini demonstrated that the amount of projecting neurons is directly correlated with the number of surviving neurons (Hamburger \& Levi-Montalcini, 1949). This observation formed the basis of the neurotrophin theory suggesting that immature neurons compete for limiting amounts of target-derived neurotrophic factors. Only those neurons survive, which are able to establish proper synaptic contacts to obtain neurotrophic support, while the remaining neurons subsequently undergo programmed cell death (Cowan et al, 1984). Consequently in sympathetic neurons, withdrawal of NGF induces the release of cytochrom $c$ into the cytosol, activation of caspases and subsequent cell death (Deshmukh \& Johnson, 1998).

\subsubsection{Induction of apoptosis in mature neurons}

While the lack of trophic support induces programmed cell death in developing neurons, apoptosis in mature neurons can be induced by several other stimuli. There are two main caspase-activation pathways - the extrinsic or death receptor-mediated pathway and the intrinsic pathway that involves the release of cytochrom c (Figure 3).

\subsubsection{Apoptotic stimuli and signaling cascades}

Extrinsic apoptosis can be induced by the activation of plasma membrane 'death receptors' of the tumor necrosis factor (TNF) receptor family, which include Fas/CD95/Apo1, TNF-receptor 1 (TNFR1), death receptor 3 (DR3), TRAIL-R1/DR4, TRAIL2/DR5 and DR6. Members of this family are characterized by extracellular cysteine-rich repeats and an intracellular death domain (DD) that is important for the transduction of the apoptotic signal (Walczak \& Krammer, 2000). Extracellular ligands belong to the TNF superfamily of cytokines including TNF- $\alpha$, Fas and Trail. After ligand binding, TNF-induced apoptosis is mediated by the recruitment of specific adapter proteins and procaspase- 8 to the death domain, thus forming a death-inducing signaling complex (DISC) (Kischkel et al, 1995; Medema et al, 1997; Scaffidi et al, 1997). Activation of high levels of caspase-8 by autoactivation within the DISC complex leads to direct activation of caspase-3, while low amounts of caspase- 8 require an additional amplification loop that includes translocation 
of truncated (t)BID to the mitochondria, release of cytochrom c, apoptosome formation and subsequent activation of caspase-3 (Cryns \& Yuan, 1998; Esposti, 2002; Kischkel et al, 2001; Micheau \& Tschopp, 2003; Schutze et al, 2008). Furthermore, activation of Fas or TNFR1 also activates the c-Jun N-terminal kinase (JNK)-death pathway inducing increased expression of BIM, p53 and FAS/FASL (Ip \& Davis, 1998; MacEwan, 2002). In addition to the TNF family of cytokines, a diverse array of acute or sub-acute stimuli including hypoxia, oxidative stress, DNA damage, ribonucleotide depletion, oncogene activation or survival factor deprivation result in mitochondrial damage and subsequent apoptosis. One of the most important sensors of cellular stress is the p53 tumor suppressor protein, a nuclear phosphoprotein that functions as a key regulator of DNA repair, cell cycle progression and apoptosis (Giaccia \& Kastan, 1998; Ko \& Prives, 1996). In response to DNA damage, initiation of the apoptotic signaling pathway involves the PI3K-like kinases ataxiatelangiectasia-mutated (ATM), ataxia-telangiectasia Rad3-related (ATR) and DNA-PKs, which in turn activate downstream checkpoint kinases like Chk1 or Chk2. These checkpoint kinases then phosphorylate p53 and its inhibitor human double minute 2 (HDM2). Activated p53 induces transcriptional upregulation of several pro-apoptotic Bcl-2 family members like Puma, Noxa, Bax and Bid, which is accompanied by transcriptional downregualtion of Bcl-2 and Bcl-X $\mathrm{X}_{\mathrm{L}}$ (Jeffers et al, 2003). In addition, p53 affects mitochondrial integrity leading to the release of cytochrom $\mathrm{c}$ and apoptosis.

\subsubsection{Apoptosis in neurodegenerative diseases}

Neuronal apoptosis of selective neurons in the brain is a hallmark of various neurodegenerative diseases, injury or insult. Several pathogenic stimuli have been identified to induce neuronal cell death in mature neurons. In stroke and seizure, accumulation of high levels of glutamate induces neurons to undergo excitotoxic cell death (Coyle \& Puttfarcken, 1993). Furthermore, exposure of human neuronal cells to the amyloid $ß$ peptide $(A ß)$ triggers apoptosis through activation of Bax and subsequent release of cytochrom c (Zhang et al, 2002). In Alzheimer's disease, $A ß$ is the major component of senile plaques in the brain of AD patients (Selkoe, 1989). Aß is produced by proteolytic cleavage of the amyloid precursor protein (APP) by beta-and gamma-secretase (Selkoe, 1998). Interestingly, various Aß species with increasing C-terminal length ranging from $A ß_{1-40}$ to $A \beta_{1-50}$ have been found in the brains of $A D$ patients (Miravalle et al, 2005). Recently, Saito and colleagues showed that $A ß_{1-43}$ has a higher toxicity as $A ß_{1-40}$ and $A ß_{1-42}$ 
(Saito et al, 2011). Conversely, also N-terminal modified and truncated forms of $A ß$ have been identified. Pyroglutamate-modified $A ß_{3-42}\left(A \beta_{3(\mathrm{pE})-42}\right)$ and truncated $A \beta_{4-42}$ are the most aboundant forms, in addition to $A ß_{1-40}$ and $A ß_{1-42}$, found in the hippocampus and cortex of AD brains (Portelius et al, 2010). Wirths and colleagues showed that intraneuronal accumulation of $\mathrm{A}_{3}(\mathrm{pE})-42$ in the hippocampus and Purkinje cells of the cerebellum leads to cerebellar atrophy, neurological impairment and growth retardation in TBA2 transgenic mice (Wirths et al, 2009). This further supports the modified amyloid hypothesis (Wirths et al, 2004), highlighting the role of intraneuronal $A ß$ in the pathological events seen in $\mathrm{AD}$, especially in neuronal cell death.

Neuronal apoptosis in neurodegenerative diseases is also caused by signals within the cell. Aggregates of misfolded proteins, which are believed to harbor toxic properties, are major hallmarks of Parkinson's disease (PD) or polyglutamine diseases (Perutz \& Windle, 2001; Schulz \& Dichgans, 1999; Taylor et al, 2002; Zoghbi \& Orr, 2000). In PD, which is the second most common age-related neurodegenerative disease, Lewy bodies composed of aggregated and misfolded $\alpha$-synuclein are formed (Spillantini et al, 1997). Another trigger of apoptosis is oxidative stress. ROS induce mitochondrial failure and DNA damage and are thus implicated in acute and chronic neurodegeneration including stroke, PD and amyotrophic lateral sclerosis (ALS) (Carri et al, 2003; Jenner, 2003; Smith et al, 2000). Strikingly, mutations of genes in inherited neurodegenerative diseases provide valuable insight into mechanisms of cell death and onset of neurodegeneration. Thus, it is crucial to delineate molecular mechanisms in developmental and disease-related neuronal apoptosis to develop new approaches to prevent and treat neurodegenerative disorders.

\subsection{Ubiquitination in neurons}

Neuronal development is regulated by a plethora of different signaling molecules coordinated in a temporal manner. Here, the ubiquitin-proteasome system (UPS) has emerged as a key regulator of neuronal development and has been implicated in several neurodegenerative diseases (Kawabe \& Brose, 2011; Yi \& Ehlers, 2007). 


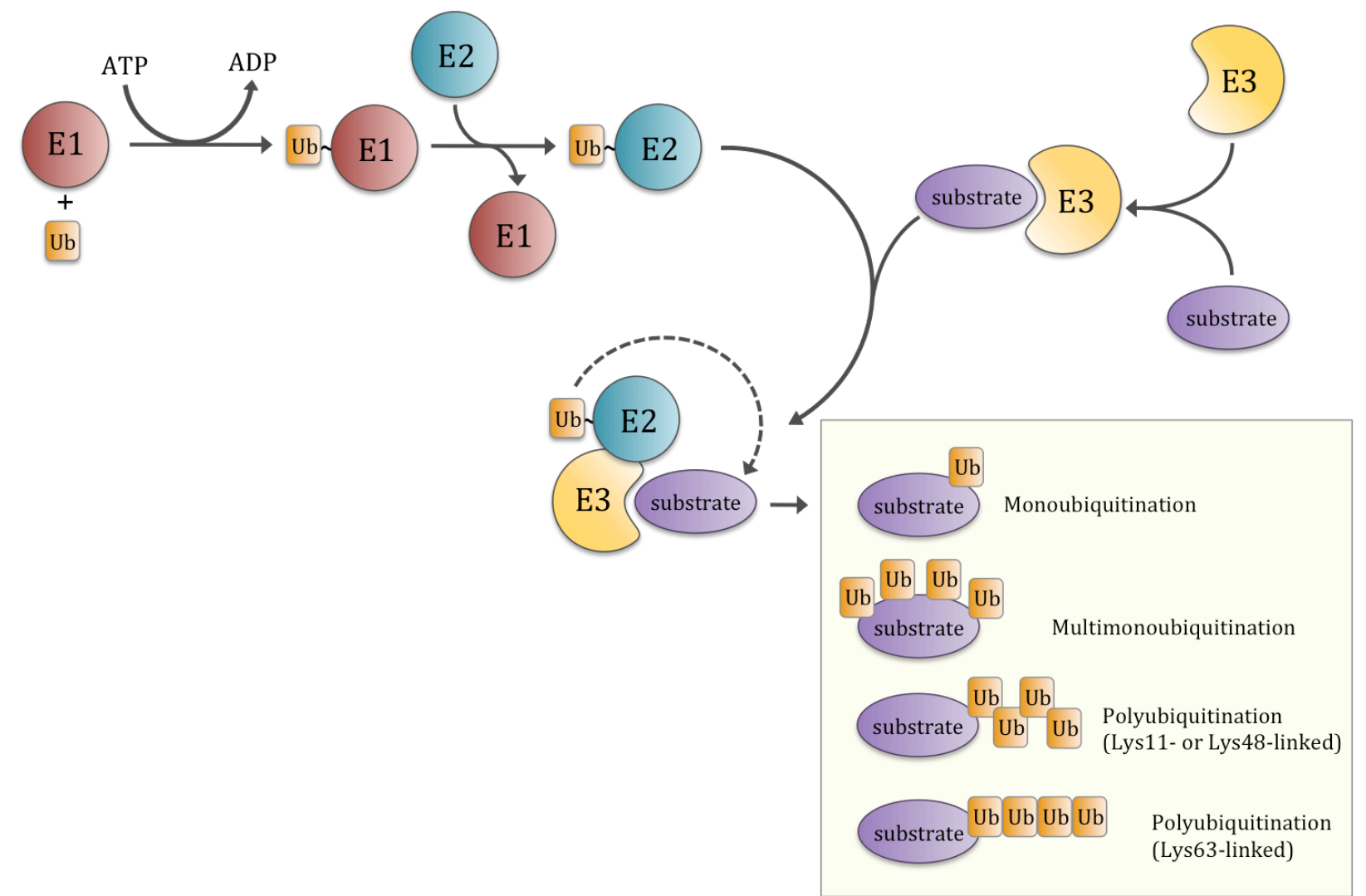

Figure 4. The ubiquitin-proteasome system. Ubiquitination of target proteins involves the sequential action of three different classes of enzymes (E1, E2 and E3). In an ATP-dependent manner, ubiquitin is activated by the E1 ubiquitin-activating enzyme and covalently bound to the E1 active site. The activated ubiquitin is then transferred to an E2 ubiquitin-conjugating enzyme. The E2 ubiquitin complex is in turn recognized by the E3 ubiquitin ligase, which mediates the transfer of ubiquitin to the substrate. Substrates can either be monoubiquitinated, multimonoubiquitinated or polyubiquitinated. While Lys11-and Lys48linked polyubiquitin chains are directly recognized by the proteasome, Lys63-linked polyubiquitin chains as well as mono-or multimonoubiquitination regulate the localization, function or interaction of proteins (modified from Kawabe and Brose, 2011). Ub, ubiquitin.

Post-translational modification of target proteins with one or more molecules of ubiquitin is the result of a multi-step process, catalyzed by the sequential action of three different classes of enzymes (Hershko \& Ciechanover, 1998), (Figure 4). First, ubiquitin is activated by the E1 ubiquitin-activating enzyme in an ATP-dependent manner. The Cterminal glycine residue of ubiquitin is covalently bound via a thioester linkage to a cysteine residue at the $\mathrm{E} 1$ active site. Activated ubiquitin is subsequently transferred to an E2 ubiquitin-conjugating enzyme. E3 ubiquitin ligases recruit both the E2-ubiquitin complex and the substrate and mediate the transfer of ubiquitin to the substrate protein (Hershko \& Ciechanover, 1998; Yi \& Ehlers, 2007). A target protein can either be monoubiquitinated, multi-monoubiquitinated or polyubiquitinated. In addition, linkage of ubiquitin to the substrate and chain elongation can occur via different lysine residues of ubiquitin (Lys6, Lys11, Lys27, Lys29, Lys33, Lys48 and Lys63). While substrates modified 
by Lys11-or Lys48-linked ubiquitin chains are recognized and degraded by the $26 \mathrm{~S}$ proteasome, Lys63-linked polyubiquitin chains as well as monoubiquitination and multimonoubiquitination regulate the localization, function or interaction of proteins (Komander \& Rape, 2012). Thus, the fate of a substrate protein is defined by the nature of its ubiquitin modification.

\subsubsection{RING E3 ligases in focus}

In mammals, there are two E1 enzymes, approximately 35 E2 enzymes and over 600 E3 ligases (Deshaies \& Joazeiro, 2009), which confer substrate specificity (Huang et al, 1999). Interestingly, E3 ligases can have more than one substrate; one substrate in turn can be targeted by different E3 ligases (Amati, 2004; Nishitani et al, 2006). There are two major families of E3 ligases, which can be distinguished by the presence of either a conserved homologous to E6AP carboxyl terminus (HECT) or a really interesting new gene (RING) domain. These domains are intimately connected with the mode of the E3 ligase activity: while HECT E3 ligases exhibit enzymatic activity to bring about ubiquitination of the substrate, RING E3 ligases act as scaffolds by facilitating the interaction between E2s and substrates (Rotin \& Kumar, 2009).
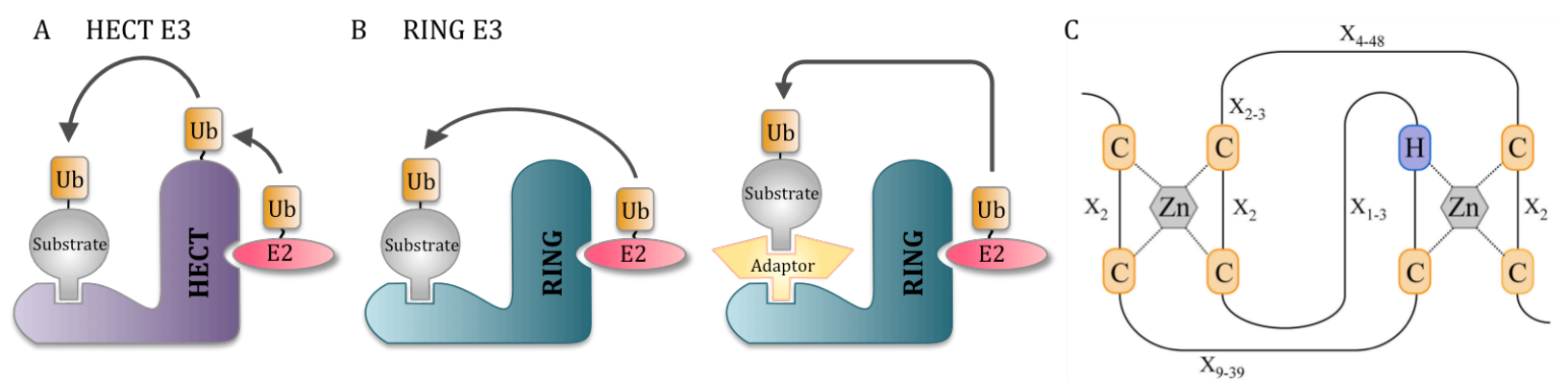

Figure 5. Structure of HECT and RING E3 ubiquitin ligases. There are two major groups of E3 ubiquitin ligases, the homologous to E6AP carboxyl terminus (HECT)-and really interesting new gene (RING)-type E3 ligases. These two groups differ in their way to transfer ubiquitin to the substrate. A) HECT E3 ligases exhibit enzymatic activity and covalently bind ubiquitin by a conserved catalytic cysteine in the HECT domain during the transfer of ubiquitin from the E2 to the substrate. B) RING E3 ligase can act as single subunit or multimeric ubiquitin ligases and confer substrate specificity to the ubiquitination system. They act as scaffolds by facilitating the transfer of ubiquitin between E2s and substrates and do not have enzymatic activity. C) The RING domain is a type of zinc finger domain, which is characterized by a defined sequence of cysteines and histidines $\left(\mathrm{CX}_{2} \mathrm{CX}_{9-39} \mathrm{CX}_{1-3} \mathrm{HX}_{2-38}(\mathrm{C} / \mathrm{H}) \mathrm{X}_{2} \mathrm{CX}_{4-48} \mathrm{CX}_{2} \mathrm{C}\right.$; $\mathrm{X}$ can be any amino acid) that coordinates two zinc ions in a cross-brace arrangement (modified from Rotin and Kumar, 2009). Ub, ubiquitin; E2, E2 ubiquitin-conjugating enzyme; C, cysteine; H, histidine; Zn, zinc.

RING E3 ligases display the largest group of E3 ligases with more than 600 different RING E3 ligases expressed in humans. The RING domain is a type of zinc finger domain that is 
characterized by a defined sequence of cysteines and histidines $\left(\mathrm{CX}_{2} \mathrm{CX}_{9-39} \mathrm{CX}_{1-3} \mathrm{HX}_{2-}\right.$ ${ }_{38}(\mathrm{C} / \mathrm{H}) \mathrm{X}_{2} \mathrm{CX}_{4-48} \mathrm{CX}_{2} \mathrm{C}$; $\mathrm{X}$ can be any amino acid) that coordinate two zinc ions in a crossbrace arrangement (Freemont, 1993). The RING class of E3 ligases can be further divided into the plant homeobox domain (PHD) and U-box families, which harbor small modifications in their RING domain. While PHD domains, sharing a similar pattern of cysteines and histidines with RING E3 ligases, are not involved in ubiquitination (Aravind et al, 2003; Scheel \& Hofmann, 2003), U-box domains, structurally related to the RING domain, exhibit E3 ligase activity (Aravind \& Koonin, 2000). The ability of the RING domain to mediate the transfer of ubiquitin depends on the direct interaction with the E2 (Zheng et al, 2000). The attachment of the first ubiquitin (chain initiation) on a substrate lysine by the E3 RING ligase and their E2 can occur randomly or in a chain initiation motifdependent manner (Williamson et al, 2011). Elongation of the chain, which requires the formation of an ubiquitin-ubiquitin isopeptide bond, can be performed by the same E2 or in cooperation with a specific chain-elongation E2 (Deshaies \& Joazeiro, 2009). While Ube2S specifically assembles ubiquitin by Lys11-linkage, Ube2R1 synthesizes Lys48-linked ubiquitin chains (Petroski \& Deshaies, 2005; Williamson et al, 2009; Wu et al, 2010). Hence, the interaction of RING E3 ligases with a specific E2 determines the nature of substrate ubiquitination.

\subsubsection{RING E3 ligases in neuronal development}

Over the last decade, RING E3 ligases have emerged as key players in neuronal development, including neurogenesis, migration, axon and dendrite growth and synapse formation (for reviews see: (Kawabe \& Brose, 2011; Tuoc \& Stoykova, 2010; Yi \& Ehlers, 2007)).

The generation of neurons early during embryonal development is regulated by a complex network of highly ordered signaling events. This involves the transcription factor Pax6, which is a member of the Pax family of transcription factors that harbor a DNAbinding paired-box and homeobox domain. Pax6 is highly expressed in RGCs and influences the generation of neuronal cells by controlling cell cycle parameters (Englund et al, 2005; Quinn et al, 2007; Schmahl et al, 1993). Tuoc and Stoykova identified the RING E3 ligase Trim11 as an interactor of Pax6. Moreover, Trim11 ubiquitinates Pax6 and regulates its degradation by the proteasome in cortical progenitors. In addition, Trim11 is able to remove insoluble Pax6. Overexpression of Trim11 reduces endogenous Pax6 protein and 
subsequently inhibits neurogenesis, while RNAi-mediated knockdown of Trim11 leads to an increase of Pax6 inclusion bodies followed by cortical progenitor cell death. Interestingly, the expression of Trim11 is controlled by Pax6 (Tuoc \& Stoykova, 2008). In this model, the functional activity of Pax6 during neurogenesis is directly linked to its regulation by Trim11.

After neurogenesis, neurons travel long distances to reach their final target position. Famulski and colleagues found that the exit of cerebellar granule neurons from their germinal zone niche is regulated by proteasomal degradation of Pard3A by the RING E3 ligase Seven in Absentia homolog (Siah). In addition, electroporation of the external granule layer (EGL) at P8 with Siah1B or Siah2-specific RNAis revealed an increase in cerebellar granule neuron (CGN) migration towards the internal granule layer (IGL) for Siah2-RNAi, but not Siah1B RNAi-transfected cells. However, overexpression of both Siah1B and Siah2 blocked CGN migration, which was also shown for Pard3A-RNAi transfected CGNs. The authors further showed that Pard3A binding to the junction adhesive molecule $\mathrm{C}$ (JAM C) is required for the contact-dependent integration of migrating neurons into the cerebellar cortex. Thus, Siah E3 ligases control germinal zone exit and neuronal migration towards the IGL by regulation of Pard3A protein levels and subsequently the interaction with JAM C (Famulski et al, 2010).

The E3 ubiquitin ligase anaphase-promoting complex (APC) and its associated regulatory subunit Cdh1 were shown to play a critical role during axon morphogenesis and patterning of the mammalian brain (Konishi et al, 2004). Cdh1-APC which was originally identified as cell cycle regulator (Stegmuller \& Bonni, 2005) is also highly expressed in the nucleus of postmitotic neurons (Gieffers et al, 1999). Here, Cdh1-APC exerts its function as E3 ubiquitin ligase regulating the degradation of several target proteins, including SnoN and the inhibitor of DNA binding Id2 (Lasorella et al, 2006; Stegmuller et al, 2006). SnoN, a member of the Ski/SnoN family of transcriptional corepressors (Stroschein et al, 1999), regulates axon growth in postmitotic neurons through transcriptional induction of the actin-binding protein Ccd1 (Ikeuchi et al, 2009). Ubiquitination of SnoN by Cdh1-APC stimulates the degradation of SnoN by the proteasome and thereby inhibits axon outgrowth (Stegmuller et al, 2006). This is further controlled by the TGFß-Smad2 signaling pathway that recruits SnoN to the Cdh1-APC-complex, thereby leading to its subsequent degradation (Stegmuller et al, 2008). 
Another example is the E3 ligase Cul7 Fbxw8 that was shown by Litterman and colleagues to regulate dendrite patterning and Golgi apparatus morphology by ubiquitination and degradation of Grasp65. Cullin RING-type E3 ligases employ F-box proteins as substrate specificity factors. Fbxw8 that is localized at the Golgi apparatus specifically interacts with Cullin7. While knockdown of Fbxw7 not only impairs dendrite growth but also normal morphology of the Golgi apparatus, knockdown of the Golgi stacking protein Grasp65 enhances dendrite growth and elaboration. Thus, regulation of the secretory pathway by the RING E3 ligase Cul7Fbxw8 identified another important mechanism in the control of dendrite morphogenesis (Litterman et al, 2011). These examples demonstrate the pivotal role of RING E3 ligases in neuronal development.

\subsubsection{RING E3 ligases in neuronal apoptosis and neurodegenerative diseases}

Neuronal apoptosis is important for proper brain formation and has potential roles in neurodegenerative diseases. Modification of apoptotic proteins by the attachment of ubiquitin regulates key components in cell death-signaling pathways. Several RING E3 ligases have been implicated in the control of neuronal apoptosis, including Cdh1-APC, Siah1, Trim17, parkin or Mgrn1.

Cdh1-APC function is important during cell cycle as well as axonal morphogenesis (Konishi et al, 2004; Sudakin et al, 1995). During cell cycle, Cdh1-APC ubiquitinates and degrades key mitotic regulators such as cyclin B1 or securin, thereby controlling together with Cdc20-APC the timely transitions of cell cycle phases. This raised the question if Cdh1APC also controls the degradation of cyclins in postmitotic neurons. Here, Almeida and colleagues showed that Cdh1-APC prevents re-entry of postmitotic neurons into the cell cycle by degradation of cyclin B1. In addition, the authors found that Cdh1 loss-of-function triggers cyclin-B1-dependent entry into $S$ phase, which leads to neuronal cell death (Almeida et al, 2005).

Another inducer of neuronal apoptosis is the RING E3 ligase Siah1. Overexpression of Siah1 in cultured sympathetic neurons increases cell death, while knockdown of Siah1 upon NGF deprivation decreases neuronal apoptosis. Xu and colleagues proposed a model, in which Siah1 is stabilized upon apoptotic stimuli through interaction with POSH (plenty of $\mathrm{SH}_{3} \mathrm{~s}$ ), a scaffold component of the JNK pathway. Subsequently, Siah1 activates the JNK pathway to influence the neuronal cell death machinery. Importantly, the induction of neuronal apoptosis is dependent on the E3 ligase activity of Siah1 (Xu et al, 2006). 
The RING E3 ligase Trim17 was identified as a critical regulator of the intrinsic apoptotic pathway. Trim17 is a member of the tripartite (TRIM) family and harbors in addition to a RING domain, a B-box type 1 and a B-box type 2, and a coiled-coil domain. While knockdown of Trim17 in KCl-deprived primary neurons almost completely blocks cell death, overexpression induces apoptosis in neurons. In contrast, expression of a Trim17 mutant lacking the RING domain has no effect on neuronal cell death. Thus, the E3 ligase activity of Trim17 is required for regulation of neuronal apoptosis (Lassot et al, 2010).

Another example is the E3 ligase parkin. Mutations in the Parkin gene have been identified as the cause of familial Parkinsonism (Mata et al, 2004). Parkin mediates the ubiquitination of itself and many other target proteins including $\alpha$-SYN-interacting protein synphilin-1, cyclin E or $\alpha / \beta$ tubulin (Ali et al, 2011). The importance of parkin's function becomes evident as it maintains neuronal viability by protection against different apoptotic stimuli such as $\alpha$-synuclein toxicity (Petrucelli et al, 2002), proteasomal dysfunction (Petrucelli et al, 2002) or endoplasmatic reticulum stress (Takahashi et al, 2003). Disruption of parkin function is thought to induce accumulation of parkin and its target substrates and ultimately the apoptosis of dopaminergic neurons (McNaught et al, 2002; Moore \& Goldberg, 2011; Petrucelli \& Dawson, 2004). In addition, the RING E3 ligases dorfin and CHIP are involved in the progression of PD. While dorfin ubiquitinates $\alpha$ synphilin-1 and localizes within Lewy bodies (Imai et al, 2002; Ito et al, 2003), CHIP enhances the E3 ligase activity of parkin (Imai et al, 2002).

Furthermore, the importance of ubiquitination becomes apparent in mice lacking the RING E3 ligase Mahogunin ring finger-1 (Mgrn1), resulting in progressive spongiform neurodegeneration as well as abnormal patterning of the left-right (LR) axis and a defect in pigment type switching (Cota et al, 2006; He et al, 2003; Miller et al, 1997; Phan et al, 2002). Interestingly, Jiao and colleagues reported that RNF157 (Mgrn2) is a homologue of the E3 ligase Mgrn1. They found a 69\% homology between RNF157 and Mgrn1 with the highest similarities at the N-terminal region (Jiao et al, 2009). In contrast, while Mgrn1 expression is detectable in the brain as well as in heart, kidney and liver, the Cerebellar Development Transcriptome Database (CDT-DB) revealed that RNF157 is a braindominant protein. However, the function of RNF157 in the central nervous system remains elusive. 


\subsubsection{RNF157 and its novel interactor Fe65}

Fe65, which was identified as a novel interactor of RNF157, represents together with Fe65L1 and Fe65L2 a family of multimodular adaptor/scaffolding proteins. The three family members are encoded in humans by separate genes on chromosome 11, 4 and 5, respectively (Blanco et al, 1998; Bressler et al, 1996; Tanahashi \& Tabira, 1999). Interestingly, Fe65 is the only family member whose expression is enriched in the mammalian brain (Duilio et al, 1991). Here, two splicing variants of Fe65 are produced by alternative splicing of a $6 \mathrm{bp}$ mini-exon. While the Fe65 isoform containing these 2 additional amino acids inserted into the first phosphotyrosine binding (PTB) domain is only expressed in neuronal cells, the expression of the second isoform without the miniexon is restricted to non-neuronal cells ( $\mathrm{Hu}$ et al, 1999). Fe65L1 and Fe65L2 are more widely expressed (Duilio et al, 1998; Guenette et al, 1999; Tanahashi \& Tabira, 1999).

The Fe65 protein family shares three conserved protein domains including one WW and two phosphotyrosine binding domains (PTB) in their C-terminal region (Ermekova et al, 1997; Fiore et al, 1995). All three family members interact with the amyloid precursor protein (APP) intracellular domain (AICD) through their second PTB domain in a Tyr682 phosphorylation-dependent manner (Borg et al, 1996; Duilio et al, 1998; Fiore et al, 1995; Guenette et al, 1996; Zhou et al, 2009). The interaction of Fe65 and APP affects APP trafficking and secretion of Aß (Fiore et al, 1995; McLoughlin \& Miller, 2008). Although all Fe65 family members can complex with AICD and translocate as a complex into the nucleus, only Fe65 harbors transcriptional activity.

The first PTB domain of Fe65 recognizes an NPXY motif found in the intracellular domain of the cell surface lipoprotein receptors LRP1 and ApoER2. Both receptors are connected with APP via Fe65 and facilitate a mechanism by which Fe65 modulates APP trafficking and signaling (Hoe et al, 2006; Trommsdorff et al, 1998).

The WW domain of Fe65 binds to proline-rich sequences as found in Mammalian enabled (Mena) or the tyrosine kinase c-Abl. Mena, a regulator of actin dynamics, colocalizes with Fe65 and APP in neuronal growth cones and synaptic terminals. Interestingly, the trimeric complex of Mena, Fe65 and APP influences axon branching, cell motility and neuronal growth cone formation (Ermekova et al, 1997; Ikin et al, 2007; Perkinton et al, 2004; Sabo et al, 2001; Sabo et al, 2003).

Recent studies have begun to characterize the role of Fe65 in vivo. In C. elegans, Fe65 displays a putative role in survival, since functional deletion of FEH-1, the single Fe65 
orthologue in worms, causes embryonic lethality or developmental arrest of the mutant worms in late embryogenesis or the first larval stage (Zambrano et al, 2002). Conversely, due to functional redundandency, the isoform-specific Fe65 as well as the Fe65L1 knockout mouse show neither cell death nor neuroanatomical abnormalities. However, characterization of Fe65/Fe65L1 double knockout mice revealed an increase in embryonic lethality and developmental brain abnormalities in cortex and hippocampus including focal heterotopias, midline crossing defects and axonal pathfinding abnormalities (Guenette et al, 2006). Interestingly, the Fe65/Fe65L1 double knockout phenotype resembles the phenotype seen in APP/APLP1/APLP2 or Mena knockout mice suggesting an important role for Fe65 together with APP and Mena in brain development (Herms et al, 2004; Lanier et al, 1999).

\subsection{Aims of the study}

The aim of my study was to unravel the role of the uncharacterized RING E3 ligase RNF157 in the brain. A previous study provided a novel link between aberrant ubiquitination and spongiform neurodegeneration in Mgrn1 knockout mice (He et al, 2003). Interestingly, Mgrn1 shares a high level of similarities with RNF157 (He et al, 2003; Kim et al, 2007a). While Mgrn1 is expressed in the brain as well as in other tissues, the Cerebellar Development Transcriptome (CDT)-Database revealed that RNF157 is a braindominant protein. However, the function of RNF157 in the brain remains elusive. To this end, my first aim was to characterize the expression and subcellular localization of RNF157 in the developing CNS using different biochemical and immunochemical methods. Subsequently, I elucidated the function of RNF157 in neurons using loss-and gain-offunction analyses. In addition, to understand the mechanism underlying RNF157 function, I identified an interactor of RNF157 and established an RNF157-dependent pathway. Beyond these in vitro analyses, I generated an RNF157 knockout mouse model to elucidate the role of RNF157 in vivo. Hence, my study improved the understanding of RING finger proteins in the mammalian brain and provided novel insight into the role of the UPS in CNS development and disease. 


\section{Materials and Methods}

\subsection{Chemicals and reagents}

Chemicals and reagents used in this work were purchased from AppliChem (Darmstadt, Germany), Roth (Karlsruhe, Germany), Merck (Darmstadt, Germany), Becton Dickinson and company (Heidelberg, Germany), Sigma-Aldrich (Steinheim, Germany) or Invitrogen (Darmstadt, Germany). If they were purchased from other companies, it will be indicated in the text. Applied protease inhibitors used in various protocols were Dithiothreitol (DTT, $1 \mathrm{mM})$, Pepstatin $(1 \mu \mathrm{g} / \mathrm{ml})$, Leupetin $(1 \mu \mathrm{g} / \mathrm{ml})$ and aprotinin (3 $\mu \mathrm{g} / \mathrm{ml})$.

\subsection{General equipment}

Plastic ware such as $1.5 \mathrm{ml}$ micro centrifuge tubes, pipet tips, reaction tubes (15 or $50 \mathrm{ml}$ ) or culture dishes were supplied by Eppendorf (Hamburg, Germany), Sarstedt AG (Nürnbrecht, Germany) or Greiner (Solingen, Germany); fluorescence microscopic images were taken with Leica confocal microscope SP2 or Eclipse Ti microscope by Nikon ${ }^{\circledR}$.

Power Pack P25T

Thermocycler T Professional Basic Gradient

Eppendorf centrifuge 5424

Eppendorf centrifuge 5804

Rocking Duomax 1030 Shaker

Grant Heater Block QBD2

Inverted light microscope Eclipse TS100

Dissection microscope
Biometra

Biometra

Eppendorf

Eppendorf

Heidolph

Thermo Scientific

Nikon

SMZ645 Nikon 


\subsection{Enzymes}

\begin{tabular}{l|l}
\hline Enzymes & Obtained from \\
\hline Pfu DNA polymerase & Fermentas \\
Restriction Enzymes + Buffer & NEB \\
Alkaline phosphatase, calf intestinal (CIP) & NEB \\
T4 DNA Ligase & Fermentas \\
T4 DNA Polymerase & Fermentas \\
T4 Polynucleotide Kinase & Fermentas \\
PCR SuperMix & Invitrogen \\
GoTap ${ }^{\circledR}$ DNA polymerase & Fermentas
\end{tabular}

\subsection{Buffers and solutions}

The buffers and solutions listed bellow were frequently used in various protocols:

TX-100 Lysis buffer: 150 mM NaCl, 50 mM Tris HCl pH 7.5, 1 mM EDTA, 1\% Triton X-100

Running buffer: 25 mM Tris, $250 \mathrm{mM}$ glycine, 0.1\% SDS

Transfer buffer: 153 mM glycine, 20 mM Tris, 20\% methanol

4xSample buffer: $25 \mathrm{ml}$ Upper Buffer, $20 \mathrm{ml}$ Glycerol, $4 \mathrm{~g}$ SDS, $2 \mathrm{ml}$ 2-Mercaptoethanol, $2 \mathrm{~g}$ Bromphenolblue (stored in $1 \mathrm{ml}$ aliquots at $-20^{\circ} \mathrm{C}$ )

Lower buffer: $1.5 \mathrm{M}$ Tris, $0.4 \%$ SDS

Upper buffer: $0.5 \mathrm{M}$ Tris, $0.4 \%$ SDS

PBS: $136 \mathrm{mM} \mathrm{NaCl}, 2.68 \mathrm{mM} \mathrm{KCl}, 4.29 \mathrm{mM} \mathrm{Na}_{2} \mathrm{HPO}_{4}$ x 7 $\mathrm{H}_{2} \mathrm{O}, 1.47 \mathrm{mM} \mathrm{KH}_{2} \mathrm{PO}_{4}$

Wash buffer: PBS $+0.1 \%$ Tween 20

2xYT media (1L): 16 g tryptone, 10 g yeast extract, $5 \mathrm{~g} \mathrm{NaCl}$

GTE buffer: 50 mM glucose, 10 mM EDTA pH 8. 5, 25 mM Tris-HCl 
Bacterial lysis buffer: $0.2 \mathrm{~N} \mathrm{NaOH}, 1 \%$ SDS

Neutralization buffer: 3 M KOAc, 11.5\% glacial acetic acid

Annealing buffer: 100 mM KAcet, 30 mM Hepes, 2 mM MgAcet

8\% PFA: Heat up $375 \mathrm{ml}$ sterile $\mathrm{H}_{2} \mathrm{O}$ to $\sim 60^{\circ} \mathrm{C}$; add $40 \mathrm{~g}$ of PFA, $50 \mathrm{ml} 10 \mathrm{x}$ PBS and $40 \mathrm{~g}$ sucrose; fill up with sterile $\mathrm{H}_{2} \mathrm{O}$ to final volume of $500 \mathrm{ml}$, pH 7.4

Co-IP buffer: 1\% NP40, 150 mM NaCl, 20 mM Tris pH 7.4, 1 mM EDTA, 10\% Glycerol

RIPA buffer: 50 mM Tris-HCl pH 8.0, $150 \mathrm{mM} \mathrm{NaCl,} \mathrm{1 \%} \mathrm{NP40,} \mathrm{0.5 \%} \mathrm{Sodium} \mathrm{deoxycholate,}$ $0.1 \%$ SDS, 5 mM EDTA

SCF buffer A: 10 mM HEPES pH 7.9, 10 mM KCl, 0.1 mM EGTA, 0.1 mM EDTA

SCF buffer B: 20 mM Hepes pH 7.9, 400 mM NaCl, 1 mM EDTA, 1 mM EGTA

Mounting media: $50 \%$ Glycerol, 5\% NPG, $\mathrm{H}_{2} \mathrm{O}$

Cbc medium: BME [+] Earle's [-] L-Glutamine, 10\% Calf serum (heat inactivated) (Hyclone), 1\% PSG and $25 \mathrm{mM} \mathrm{KCl}$

BME112 medium: $35 \mathrm{mM}$ Glucose, $10 \mu \mathrm{g} / \mathrm{ml}$ Insulin, 1\% PSG in BME [+] Earle's [-] LGlutamine

2xHBSS: $273 \mathrm{mM} \mathrm{NaCl}, 9.5 \mathrm{mM} \mathrm{KCl}, 0.0354 \mathrm{mM} \mathrm{Na}_{2} \mathrm{HPO}_{4} \mathrm{X} \mathrm{H}_{2} \mathrm{O}, 14.8 \mathrm{mM}$ glucose and 41 mM Hepes; pH 7.05 or pH 7.08

HHGN: 10\% 10x HBSS-Hank's buffered salt solution [-] Calcium [-] magnesium, $2.5 \mathrm{mM}$ Hepes pH 7.3-7.5, $350 \mathrm{mM}$ glucose and 0.04 $\mathrm{M} \mathrm{NaHCO}_{3}$

DnB: $500 \mu \mathrm{l}$ DNase $(2 \mu \mathrm{g} / \mu \mathrm{l}), 10 \mathrm{ml} \mathrm{BME} \mathrm{[+]} \mathrm{Earle's} \mathrm{[-]} \mathrm{L-Glutamine}$

TDn: $250 \mu \mathrm{l}$ DNase $(2 \mu \mathrm{g} / \mu \mathrm{l}), 5 \mathrm{ml}$ HHGN and $50 \mathrm{mg}$ Trypsin (Worthington)

Hippocampal plating media: DMEM [+] $4.5 \mathrm{~g} / \mathrm{l}$ glucose [+] glutamine [+] pyruvate, 10\% FBS (Biochrom), 1\% PSG and 0,0125 mM glutamate 
Hippocampal growth medium: Neurobasal medium, 2\% B27 supplement and 1\% PSG

HEK 293T medium: DMEM [+] $4.5 \mathrm{~g} / \mathrm{l}$ glucose [-] glutamine [-] pyruvate with 10\% FBS and 1\% Glutamax

APC lysis buffer: 2\% TX-100, 300 mM NaCl, 100 mM Tris pH 7.2, 4 mM EDTA

QA buffer: $10 \mathrm{mM}$ Tri pH 7.5, $100 \mathrm{mM} \mathrm{KCl,} 1 \mathrm{mM} \mathrm{MgCl} 2,0,1 \mathrm{mM} \mathrm{CaCl} 2,1 \mathrm{mM}$ DTT

10x APC buffer with ATP regenerating system: $50 \mathrm{mM}$ Tris $\mathrm{pH} 7.5,10 \mathrm{mM} \mathrm{MgCl}$, $20 \mathrm{mM}$ ATP, $1 \mathrm{mM}$ EDTA, $300 \mathrm{U} / \mathrm{ml}$ creatine kinase, $75 \mathrm{mM}$ phosphocreatine, $3 \mathrm{U} / \mathrm{ml}$ pyrophosphatase

Tail Lysis buffer: 10 mM Tris pH 8.0, 10 mM EDTA, 0.5\% SDS; add fresh $200 \mathrm{mM} \mathrm{NaCl,} 200$ $\mu \mathrm{g} / \mathrm{ml}$ Proteinase $\mathrm{K}$

Blocking buffer: BME [+] Earle's [-] L-Glutamine with $10 \% \mathrm{HS}$

Hoechst: $1 \mu \mathrm{g} / \mu \mathrm{l}$ in PBS, $0.1 \%$ Triton X-100

Citrate buffer: $9 \mathrm{ml}$ of stock solution A (0.1 M citrate buffer) and $41 \mathrm{ml}$ of stock solution B (0.1 M sodium citrate buffer); add $\mathrm{H}_{2} 0$ to $500 \mathrm{ml}$

Tris buffer: $150 \mathrm{mM} \mathrm{NaCl}, 0.05 \mathrm{M}$ Tris pH 7.6

Tris-M buffer: Tris buffer, 2\% milkpowder

IHC blocking solution: $20 \%$ goat serum in PBS/BSA

\subsection{Kits}

\begin{tabular}{l|l}
\hline Kits & Obtained from \\
\hline Nucleo Spin ${ }^{\circledR}$ Plasmid Quick Pure & Macherey-Nagel \\
Nucleo Spin ${ }^{\circledR}$ Extract II & Macherey-Nagel \\
Nucleo Bond ${ }^{\circledR}$ Xtra Midi EF & Macherey-Nagel \\
TOPO TA cloning Kit & Invitrogen \\
QuickChange II Site-directed Mutagenesis Kit & Stratagene \\
SuperScript® III First-Strand Synthesis SuperMix & Invitrogen
\end{tabular}




\subsection{Vector plasmids}

\begin{tabular}{|c|c|c|}
\hline Vector plasmid & Constructed by & Primer used \\
\hline pBluescript-U6-RNF157 RNAi II & A. Matz & $14905 / 14906$ \\
\hline pBluescript-U6-RNF157 RNAi III & A. Matz & $14907 / 14908$ \\
\hline pCMV-myc-RNF157 & A. Matz & $15828 / 15829$ \\
\hline pCMV-myc-RNF157-Res & A. Matz & $22180 / 22181$ \\
\hline pCMV-myc-RNF157 $\Delta$ RING & A. Matz & $15828 / 17434 / 17435 / 15829$ \\
\hline pCMV-myc-DR6 & A. Matz & $24715 / 24716$ \\
\hline pBluescript-U6-DR6 RNAi I & A. Matz & $24711 / 24712$ \\
\hline pBluescript-U6-DR6 RNAi II & A. Matz & $24713 / 24714$ \\
\hline pBluescript-U6-DR6 RNAi III & A. Matz & $25825 / 25826$ \\
\hline pCMV-myc-Wlds * & A. Matz & $25156 / 25157$ \\
\hline pGBT9 ${ }_{\mathrm{L}}$ & A. Matz & $15019 / 15020$ \\
\hline pGBT9 $_{\mathrm{L}}-\mathrm{RNF157}$ bp892-1932 & A. Matz & $16334 / 16335$ \\
\hline pGBT9 $2-R N F 157_{\text {bp694-933 }}$ & A. Matz & $17172 / 17257$ \\
\hline pGBT9L-RNF157 bp1-921 & J. Stegmüller & $16420 / 16421$ \\
\hline pCMV-myc-Fe65 aa1-325 & J. Stegmüller & $17802 / 17803$ \\
\hline pCMV-myc-Fe65 aа326-524 & J. Stegmüller & $17804 / 17805$ \\
\hline pCMV-myc-Fe65 ${ }_{\text {aa525-711 }}$ & J. Stegmüller & $17806 / 17807$ \\
\hline pCMV-myc-Fe65 $5_{\text {aa1-5245 }}$ & J. Stegmüller & $17802 / 17805$ \\
\hline pCMV-myc-Fe65 аa326-711 & J. Stegmüller & $17804 / 17807$ \\
\hline pCMV-myc-Fe65 ${ }^{* *}$ & A. Matz & $17802 / 17807$ \\
\hline pBluescript-U6-Fe65 RNAi I & A. Matz & $19774 / 19775$ \\
\hline pBluescript-U6-Fe65 RNAi II & A. Matz & $19776 / 19777$ \\
\hline pBluescript-U6-Fe65 RNAi III & A. Matz & $19778 / 19779$ \\
\hline pEGFP-C2-RNF157 & A. Matz & $14864 / 14763$ \\
\hline pEGFP-C2-RNF157 aa1-237 & J. Stegmüller & $14864 / 17728$ \\
\hline pEGFP-C2-RNF157 aа238-442 & J. Stegmüller & $17442 / 17729$ \\
\hline pEGFP-C2-RNF157aa443-644 & J. Stegmüller & $17444 / 17730$ \\
\hline
\end{tabular}

* The Wlds cDNA sequence was kindly provided by Dr. Michael Coleman, Babraham Institute, England.

** The Fe65 cDNA sequence was kindly provided by Dr. Uwe Konietzko, University Zurich, Switzerland. 


\begin{tabular}{|l|l|}
\hline Vector plasmids & Obtained from \\
\hline cDNA-ß-galactosidase & J. Stegmüller \\
\hline pcDNA3.1-Bcl- $\mathrm{x}_{\mathrm{L}}$ & J. Stegmüller \\
\hline pCMV-myc & J. Stegmüller \\
\hline pBluescript-U6 & J. Stegmüller \\
\hline pcDNA3.1 & J. Stegmüller \\
\hline pGBT9 & J. Stegmüller \\
\hline pcDNA3.1-Flag-Fe65 & T. Suzuki \\
\hline pCMV-myc-NLS-FE65 & J. Stegmüller \\
\hline pCMV-myc-NES-FE65 & J. Stegmüller \\
\hline
\end{tabular}

\subsection{Oligonucleotides}

\begin{tabular}{|l|l|}
\hline Primer & \multicolumn{1}{|c|}{ Sequence } \\
\hline $\mathbf{1 5 9 6}$ & TCGATGATGAAGATACCCCACC \\
\hline $\mathbf{1 6 8 1}$ & AGAAATTGAGATGGTGCACGAT \\
\hline $\mathbf{1 4 7 5 8}$ & ATGGCCCAGTTGCTACGCAGCTAT \\
\hline $\mathbf{1 4 7 5 9}$ & AAGAGCGCTCATCTTCCTCTTCGT \\
\hline $\mathbf{1 4 7 6 0}$ & ATGAGCGCTCTTGCAGTGAGTCAG \\
\hline $\mathbf{1 4 7 6 1}$ & CAGACAGCCAAAGGATCCCATACG \\
\hline $\mathbf{1 4 7 9 8}$ & ATGGGAGGAGAGAAGTTTGACTGC \\
\hline $\mathbf{1 4 9 0 5}$ & AGAGGACATGCGCATTTCTAAAGTTAACGTAGAAATGCGCATGTCCTCTCTTTTG \\
\hline $\mathbf{1 4 9 0 6}$ & AATTCAAAAGAGAGGACATGCGCATTTCTACGTTAACTTTAGAAATGCGCATGTCCTCT \\
\hline $\mathbf{1 4 9 0 7}$ & GGACAATAAGCTGTGCTCTGAAGTTAACGCAGAGCACAGCTTATTGTCCCTTTTG \\
\hline $\mathbf{1 4 9 0 8}$ & AATTCAAAAGGGACAATAAGCTGTGCTCTGCGTTAACTTCAGAGCACAGCTTATTGTCC \\
\hline $\mathbf{1 5 8 2 8}$ & TATCTCGAGCCATGGGAGGAGAGAAGTTTGACTGC \\
\hline $\mathbf{1 5 8 2 9}$ & TATGCGGCCGCTCAGACAGCCAAAGGATCCCATAC \\
\hline $\mathbf{2 2 1 8 0}$ & GTCGCGAGCGTGAAAGCACTGGATAACAAACTCTGTTCGGAGGTCTGCTTACCCGGTACCTG \\
\hline $\mathbf{2 2 1 8 1}$ & GCAAC \\
\hline $\mathbf{1 7 4 3 4}$ & GCGAC \\
\hline $\mathbf{1 7 4 3 5}$ & GCCCGGAAGGGGGGCACAGGGCAGGGACACTCCGCACTGTTGTCACTGAC \\
\hline $\mathbf{2 4 7 1 5}$ & TATACTCGAGATATGGGGAGTGTCGGCTGCCCTTCCGGGC \\
\hline $\mathbf{2 4 7 1 6}$ & TATAGCGGCCGCCTACAATAGGTCAGGAAGATGG \\
\hline $\mathbf{2 4 7 1 2}$ & AATTCAAAAGAGAGCCATCTTCCTGACCTACGTTAACTTTAGGTCAGGAAGATGGCTCT \\
\hline $\mathbf{2 4 7 1 1}$ & AGAGCCATCTTCCTGACCTAAAGTTAACGTAGGTCAGGAAGATGGCTCTCTTTTG \\
\hline $\mathbf{2 4 7 1 3}$ & AAGGCATGAACTCAACAGATAAGTTAACGATCTGTTGAGTTCATGCCTTCTTTTG \\
\hline $\mathbf{2 4 7 1 4}$ & AATTCAAAAGAAGGCATGAACTCAACAGATCGTTAACTTATCTGTTGAGTTCATGCCTT \\
\hline $\mathbf{2 5 8 2 5}$ & AGAGATTACCTTGTGCTGCCAAGTTAACGGGCAGCACAAGGTAATCTCTCTTTTG \\
\hline $\mathbf{2 5 8 2 6}$ & AATTCAAAAGAGAGATTACCTTGTGCTGCCCGTTAACTTGGCAGCACAAGGTAATCTCT \\
\hline $\mathbf{2 5 1 5 6}$ & TATACTCGAGATATGGAGGAGCTGAGCGC \\
\hline $\mathbf{2 5 1 5 7}$ & TATAGCGGCCGCTCACAGAGTGGAATGGTTGTG \\
\hline $\mathbf{1 7 8 0 2}$ & ATAGTCGACAATGTCTGTTCCATCATCACTGAG \\
\hline $\mathbf{1 7 8 0 3}$ & ATAGCGGCCGCTTAGGCCTCATCACTGGGTTC \\
\hline
\end{tabular}




\begin{tabular}{|l|l|}
\hline $\mathbf{1 7 8 0 4}$ & ATAGTCGACACCAATGGAGCTGGGACTG \\
\hline $\mathbf{1 7 8 0 5}$ & ATAGTCGACACCAATGGAGCTGGGACTG \\
\hline $\mathbf{1 7 8 0 6}$ & ATAGTCGACAGATGTCCCTTTCCAAGTGGA \\
\hline $\mathbf{1 7 8 0 7}$ & ATAGCGGCCGCTCATGGGGTATGGGCCC \\
\hline $\mathbf{1 9 7 7 4}$ & AACTGGCACATCCCAACAGGAAGTTAACGCCTGTTGGGATGTGCCAGTTCTTTTG \\
\hline $\mathbf{1 9 7 7 5}$ & AATTCAAAAGAACTGGCACATCCCAACAGGCGTTAACTTCCTGTTGGGATGTGCCAGTT \\
\hline $\mathbf{1 9 7 7 6}$ & AAGATTCCTTCTGGAACCCCAAGTTAACGGGGGTTCCAGAAGGAATCTTCTTTTG \\
\hline $\mathbf{1 9 7 7 7}$ & AATTCAAAAGAAGATTCCTTCTGGAACCCCCGTTAACTTGGGGTTCCAGAAGGAATCTT \\
\hline $\mathbf{1 9 7 7 8}$ & AAGCTGACCCAGATGCTCAAAAGTTAACGTTGAGCATCTGGGTCAGCTTCTTTTG \\
\hline $\mathbf{1 9 7 7 9}$ & AATTCAAAAGAAGCTGACCCAGATGCTCAACGTTAACTTTTGAGCATCTGGGTCAGCTT \\
\hline $\mathbf{1 5 0 1 9}$ & AATTCCCCGGGAAGCTTCTCGAGGCGGCCGCGTTAACGTCGACAG \\
\hline $\mathbf{1 5 0 2 0}$ & GATCCTGTCGACGTTAACGCGGCCGCCTCGAGAAGCTTCCCGGGG \\
\hline $\mathbf{1 6 3 3 4}$ & TATGCGGCCGCCATGGCATCAATGCAGAAGCTGATCTCAGAGGAGGACCTGCTTTCTCCAAG \\
\hline $\mathbf{1 6 3 3 5}$ & CAGCTTTAACCCCATC \\
\hline $\mathbf{1 7 1 7 2}$ & AATCTGCAGTCAGACAGCCAAAGGATCCCATAC \\
\hline $\mathbf{1 7 2 5 7}$ & ATAGGATCCCTACGAAGTCTGGGAAGAGATGAT \\
\hline $\mathbf{1 6 4 2 0}$ & ATAGCGGCCGCTATGGCATCA ATGCAGAAG \\
\hline $\mathbf{1 6 4 2 1}$ & ATAGGATCCCTAAGAGATGATGGGGTTAAAG \\
\hline $\mathbf{1 4 8 6 4}$ & GGCAAGCTTCATGGGAGGAGAGAAGTTTGACTGC \\
\hline $\mathbf{1 4 7 6 3}$ & GGCGTCGACCAGACAGCCAAAGGATCCCATACG \\
\hline $\mathbf{1 7 7 2 8}$ & TATTCTAGATCACGCACTGTTGTCACTGACATCATCC \\
\hline $\mathbf{1 7 7 4 2}$ & TATAAGCTTCGAGTGTGTGGTGTGTCTCTCAGAC \\
\hline $\mathbf{1 7 7 2 9}$ & TATTCTAGATCACTCACTCTCTGGAGTCACATCAGG \\
\hline $\mathbf{1 7 4 4 4}$ & TATAAGCTTCAACCTCACGCTGTCCTCCTCAG \\
\hline $\mathbf{1 7 7 3 0}$ & GGCTCTAGATCAGACAGCCAAAGGATCCCATACG \\
\hline
\end{tabular}

\begin{tabular}{|l|l|}
\hline \multicolumn{2}{|c|}{ RNF157 Genotyping Primer } \\
\hline Primer & Sequence \\
\hline $\mathbf{2 5 1 7 1}$ & CTTGCAAAATGGCGTTACTTAAGC \\
\hline $\mathbf{2 5 1 7 2}$ & GCGCTGACATCCTGTGTTC \\
\hline $\mathbf{2 5 1 7 3}$ & GTTCTGGAATGAAGTATCCTC \\
\hline \multicolumn{2}{|c|}{ RNF157 RT-PCR Primer } \\
\hline Primer & Sequence \\
\hline $\mathbf{1 5 8 0 0}$ & TATGGATCCTTACTCTGCATCCTGCTCTCCAGCTGG \\
\hline $\mathbf{1 5 8 3 4}$ & TATCCCGGGGTTCTTGGAGATGGCCACCTCTCAGG \\
\hline $\mathbf{4 8 7 6}$ & AGGAACACGGAAGGCCATG \\
\hline $\mathbf{4 8 7 7}$ & ATGGCCCCTCTGGAAAGCT \\
\hline
\end{tabular}




\subsection{Antibodies}

Primary and secondary antibodies used for Western blotting and immunostaining in this study:

\begin{tabular}{|c|c|c|c|c|c|}
\hline \multirow{2}{*}{ Primary antibody } & \multirow{2}{*}{$\begin{array}{c}\text { Host } \\
\text { species }\end{array}$} & \multirow{2}{*}{\multicolumn{2}{|c|}{ Obtained from }} & \multicolumn{2}{|c|}{ Usage Dilution } \\
\hline & & & & WB & ICC/IHC \\
\hline$\alpha$ RNF157 & Rabbit & \multicolumn{2}{|l|}{ Sigma-Aldrich } & $1: 300$ & $1: 100$ \\
\hline$\alpha 14-3-3 ß$ & Mouse & \multicolumn{2}{|l|}{ Santa Cruz } & $1: 1000$ & \\
\hline$\alpha \mathrm{Sp} 1$ & Rabbit & \multicolumn{2}{|l|}{ Santa Cruz } & $1: 500$ & \\
\hline$\alpha$ TujI & Mouse & \multicolumn{2}{|l|}{ Sigma-Aldrich } & & $1: 1000$ \\
\hline$\alpha$ MAP2 & Mouse & \multicolumn{2}{|l|}{ Sigma-Aldrich } & & $1: 1000$ \\
\hline$\alpha$ PSD-95 & Mouse & \multicolumn{2}{|l|}{ Abcam } & & $1: 200$ \\
\hline$\alpha$ myc & Mouse & \multicolumn{2}{|l|}{ Santa Cruz } & $1: 1000$ & $1: 250$ \\
\hline$\alpha ß$-galactosidase & Mouse & \multicolumn{2}{|l|}{ Santa Cruz } & $1: 500$ & $1: 100$ \\
\hline$\alpha$ cleaved caspase- 3 & Rabbit & \multicolumn{2}{|l|}{ Cell signaling } & & $1: 200 / 1: 100$ \\
\hline$\alpha$ GFP & Rabbit & \multicolumn{2}{|l|}{ Invitrogen } & 1:1000 & 1:1000 \\
\hline$\alpha$ Fe65 & Rabbit & \multicolumn{2}{|l|}{ Santa Cruz } & $1: 1000$ & \\
\hline$\alpha$ Fe65 & Mouse & \multicolumn{2}{|l|}{ Abcam } & & $1: 100$ \\
\hline$\alpha$ Flag & Mouse & \multicolumn{2}{|l|}{ Sigma Aldrich } & $1: 1000$ & \\
\hline$\alpha$ Ubiquitin & Rabbit & \multicolumn{2}{|l|}{ Dako } & $1: 500$ & \\
\hline$\alpha$ Ubiquitin Lys48 & Rabbit & \multicolumn{2}{|l|}{ Millipore } & $1: 500$ & \\
\hline$\alpha$ Ubiquitin Lys63 & Rabbit & \multicolumn{2}{|l|}{ Millipore } & $1: 500$ & \\
\hline$\alpha$ GFAP & Mouse & \multicolumn{2}{|l|}{ Chemicon } & $1: 200$ & \\
\hline$\alpha \mathrm{HA}$ & Mouse & & & & \\
\hline \multirow{2}{*}{$\begin{array}{l}\text { Secondary } \\
\text { antibody }\end{array}$} & \multirow{2}{*}{$\begin{array}{c}\text { Host } \\
\text { species }\end{array}$} & \multirow{2}{*}{$\begin{array}{c}\text { Conjugated } \\
\text { substrate/dye }\end{array}$} & \multirow{2}{*}{$\begin{array}{l}\text { Obtained } \\
\text { from }\end{array}$} & \multicolumn{2}{|c|}{ Usage Dilution } \\
\hline & & & & WB & ICC/IHC \\
\hline$\alpha$ mouse IgG & Goat & HRP & Dianova & $1: 1000$ & \\
\hline$\alpha$ rabbit IgG & Goat & HRP & Dianova & 1:1000 & \\
\hline$\alpha$ mouse IgG & Goat & Cy2/Alexi 488 & Dianova & & $1: 1000$ \\
\hline$\alpha$ rabbit IgG & Goat & Cy2/ Alexa488 & Dianova & & $1: 1000$ \\
\hline$\alpha$ mouse IgG & Goat & Cy3/ Alexa555 & Dianova & & $1: 1000$ \\
\hline$\alpha$ rabbit IgG & Goat & Сy3/ Alexa555 & Dianova & & $1: 1000$ \\
\hline
\end{tabular}




\subsection{Protein biochemistry methods}

\subsubsection{Isolation of rodent neural and non-neural tissue}

Dissection of murine tissue was done on a sterilized laboratory bench. Mice or rats were either killed by decapitation or by the administration of $\mathrm{CO}_{2}$. Subsequently, the skull was opened and the brain was taken out. The desired brain regions such as cerebellum, cortex, hippocampus and olfactory bulb were isolated using forceps, scalpel and scoop. For non-neural tissue, the abdomen of the animal was cut anterior to posterior and the skin was fixed on the left and right site. Lung, liver, heart, kidney, spleen and testis were isolated. Tissues were either flash-frozen in liquid nitrogen or processed directly by homogenization in the appropriate lysis buffer supplemented with protease inhibitors.

\subsubsection{Preparation of rodent tissue lysates}

The murine tissues such as cerebellum, cortex, hippocampus, olfactory bulb, lung, liver, heart, kidney, spleen and testis were isolated and homogenized in respective volumes of ice-cold TX-100 buffer supplemented with protease inhibitors. Homogenization was either performed with a $2 \mathrm{ml}$ Dounce or Ultra Turrax $\left(\mathrm{IKA}^{\circledR}\right)$ depending on the tissue. Lysates were placed on ice for $30 \mathrm{~min}$ and spun for at $13000 \mathrm{rpm}$ for $10 \mathrm{~min}$ at $4^{\circ} \mathrm{C}$. The supernatant was collected and protein concentration was measured using Bradford.

\subsubsection{Preparation of HEK 293T cell lysates}

For preparation of HEK 293T cell lysates, cells were washed once on ice with PBS, which was then aspirated off and an appropriate volume of TX-100 buffer (300 $\mu$ per well of a 6 well plate) supplemented with protease inhibitors was added to the wells. Adherent cells were scraped from the culture dish using a cell scraper and lysates were collected in a $1.5 \mathrm{ml}$ eppendorf tube. Lysates were incubated on ice for $30 \mathrm{~min}$ and then spun at 13000 rpm for $10 \mathrm{~min}$ at $4^{\circ} \mathrm{C}$.

\subsubsection{Co-Immunoprecipitation of proteins}

HEK 293T cells or cultured cerebellar granule neurons were washed once with PBS, which was aspirated off. Cells or tissues were lysed in ice-cold immunoprecipitation buffer supplemented with protease inhibitors. At least $1 \mathrm{mg}$ of protein and $1 \mu \mathrm{g}$ of antibody were used per condition in any given experiment. Lysates were incubated for $30 \mathrm{~min}$ on ice and spun down at $13000 \mathrm{rpm}$ for $10 \mathrm{~min}$ at $4^{\circ} \mathrm{C} .30 \mu \mathrm{l}$ were set aside as input. The protein was 
incubated with the antibody for $4 \mathrm{hr}$ or overnight at $4^{\circ} \mathrm{C}$. Subsequently, $30 \mu \mathrm{l}$ of Protein Asepharose (Protein A-Sepharose ${ }^{\mathrm{TM}}$ Fast Flow, GE Healthcare) was added. Protein Asepharose binds rabbit as well as mouse IgGs, respectively. Before usage, beads were washed three times with Co-IP buffer. Samples were incubated with the beads for $1 \mathrm{hr}$ at $4^{\circ} \mathrm{C}$ by rotation. Thereafter, samples were centrifuged for $30 \mathrm{sec}$ at $13000 \mathrm{rpm}$. Supernatant was discarded and beads were washed twice for 5-10 min at $4^{\circ} \mathrm{C}$ either with RIPA or TX100 buffer to meet the required stringency and then spun as described above. In the last step, beads were washed with PBS, spun down and PBS was aspirated off leaving 20 $\mu$ l. Elution of the protein was performed by pipetting $30 \mu \mathrm{l}$ of sample buffer to the beads followed by boiling at $95^{\circ} \mathrm{C}$ for $5 \mathrm{~min}$. Supernatant and input were used for Western blot analysis.

\subsubsection{Subcellular fractionation of cerebellar granule neurons}

Cerebellar granule neurons (CGNs) were grown in 6 well plates for the indicated times in vitro. At least two wells were used per condition. CGNs were first washed once with PBS. Then, $300 \mu \mathrm{l}$ of detergent-free SCF buffer A was added and adherent neurons were scraped off the culture dish using a cell scraper. Lysates were incubated on ice for 15 min. Afterwards neurons were subjected to a mechanic disruption using a $2 \mathrm{ml}$ Dounce. Nuclei were spun down for $5 \mathrm{~min}$ at $2000 \mathrm{rpm}$ at $4^{\circ} \mathrm{C}$ and supernatant was used as cytoplasmic fraction (PNS). Pellet was washed three times in SCF buffer A supplemented with $0.1 \%$ NP40. Nuclei were then lysed in SCF buffer B and incubated rotating for 15 min at $4^{\circ} \mathrm{C}$. Nuclei were pelleted by centrifugation at $13000 \mathrm{rpm}$ for $20 \mathrm{~min}$ at $4^{\circ} \mathrm{C}$ and supernatant was harvested as nuclear fraction (NF) for Western blot analysis.

\subsubsection{In vitro ubiquitination assay}

Prior the in vitro ubiquitination assay, Flag-Fe65 was purified using Flag M2 affinity beads according to the manufacturer's protocol and confirmed by immunoblotting using the Flag antibody. In addition, myc-RNF157 expressed in Neuro 2A cells was immunoprecipitated for $2 \mathrm{hr}$ at $4^{\circ} \mathrm{C}$ using the myc antibody and bound to protein Asepharose beads. In the in vitro ubiquitination assay, $6 \mu \mathrm{l}$ of immunopurified myc-RNF157 was incubated at $37^{\circ} \mathrm{C}$ in a $12 \mu \mathrm{l}$ reaction volume containing $1.2 \mu \mathrm{l}$ of $10 \mathrm{x}$ APC buffer with ATP regeneration system, $0.5 \mu \mathrm{l}$ ubiquitin $(25 \mathrm{mg} / \mathrm{ml}), 0.8 \mu \mathrm{l}$ E1 activating enzyme $(0.5$ $\mathrm{mg} / \mathrm{ml}$ ), $1 \mu \mathrm{l} \mathrm{E2} \mathrm{(UbcH2,} \mathrm{UbcH3,} \mathrm{UbcH5a,} \mathrm{UbcH5b,} \mathrm{UbcH5c)} \mathrm{with} \mathrm{or} \mathrm{without} 1 \mu$ l of Flag- 
Fe65. After 90 min of incubation, the reaction products were boiled in sample buffer and analyzed by immunoblotting using the respective antibodies.

\subsubsection{Determination of protein concentration by Bradford}

Determination of protein concentration was performed using Bradford Reagent (Bio-Rad ${ }^{\circledR}$ ). The Bradford solution was diluted 1:5 in PBS (pH 7.3). For the standard 2, 4 and $8 \mu \mathrm{g}$ of BSA (bovine serum albumin) solution were diluted in $1 \mathrm{ml}$ of Bradford/PBS solution in a $1 \mathrm{ml}$ microcuvette. To analyze the protein concentration, $2 \mu \mathrm{l}$ of the lysate were diluted and mixed in $1 \mathrm{ml}$ of Bradford/PBS solution. The concentration was measured with a photometer, (Amersham Biosciences, Ultro spec 3100 pro) set to the Basis Mode, measuring absorbance $(A=-\log (\% / \mathrm{T} / 100)$ at $595 \mathrm{~nm}$.

\subsubsection{Detection of proteins by Western Blot analysis}

Lysates were diluted in sample buffer, boiled at $95^{\circ} \mathrm{C}$ for $5 \mathrm{~min}$ and spun down in a centrifuge. For analysis of protein expression $30-90 \mu \mathrm{g}$ of protein was loaded onto a SDS gel. The amount of total protein was equal in each lane. The gel consists of a stacking gel $(3,6 \%$ acrylamide) containing the wells and a separating gel with either 8 or $10 \%$ acrylamide concentration. First, all reagents of the separating gel were mixed together (Table 1) and then the required amounts of TEMED and 10\% APS were added directly before pouring the gel into the gel cast system (Mini-PROTEAN Tetra Electrophoresis System, Bio $\operatorname{Rad}^{\circledR}$ ). Gel was overlaid with isopropanol. After approximately $30 \mathrm{~min}$ of polymerisation, isopropanol was removed and the stacking gel (Table 1) was poured on the top. A 10 well comb was inserted immediately into the stacking gel. After polymerization, the protein lysates were loaded and separated in the gel by electricity $(70$ and $130 \mathrm{~V}$ ). The protein transfer was performed in a cooled wet-blot system (Mini Trans-Blot ${ }^{\circledR}$ Electrophoretic Transfer Cell, Bio Rad) at 250-300 $\mathrm{mA}$ for 60-120 min at RT onto a nitrocellulose membrane (VWR). After transfer of proteins, the membrane was blocked in wash buffer supplemented with $4 \%$ milkpowder (Frema Reform) for $30 \mathrm{~min}$ at RT. Membrane was washed three times for $10 \mathrm{~min}$ in wash buffer. Incubation with the primary antibody was performed for $1 \mathrm{hr}$ at $\mathrm{RT}$ or overnight at $4^{\circ} \mathrm{C}$ (summary of primary antibodies used see in 2.8). Membrane was washed three times in wash buffer for $10 \mathrm{~min}$ at RT and then incubated with the secondary antibody diluted in wash buffer with 4\% milkpowder for 30-45 min at RT. The horseradish peroxidase (HRP) coupled secondary antibody was directed against the species in which the primary antibody was generated in (summary of secondary antibody used see in 2.8). Membrane was washed three 
times with wash buffer. Protein bands were detected using ECL according to manufactures instructions (Pierce ECL Western Blotting Substrate, Thermo Fisher Scientific) on a chemoluminescence film (GE Healthcare). Films were developed in an automatic film processor (Kodak imaging station).

Table 1. Preparation of acrylamide gels.

\begin{tabular}{|c|c|c|c|}
\hline & \multicolumn{2}{|c|}{ Separating Gel (total volume 7 ml) } & $\begin{array}{c}\text { Stacking Gel } \\
\text { (total volume 4.95 ml) }\end{array}$ \\
\hline \% Acrylamide & $8 \%$ & $10 \%$ & $3.9 \%$ \\
\hline Acrylamide & $2 \mathrm{ml}$ & $2.5 \mathrm{ml}$ & $0.65 \mathrm{ml}$ \\
\hline Lower buffer & 1.875 & 1.875 & $1.25 \mathrm{ml}$ Upper buffer \\
\hline $\mathrm{H}_{2} \mathrm{O}$ & $3.625 \mathrm{ml}$ & $3.125 \mathrm{ml}$ & $3.05 \mathrm{ml}$ \\
\hline
\end{tabular}

\subsection{Immunocytochemistry}

\subsubsection{Perfusion of mice and generation of paraffin-embedded brain sections}

Mice were anesthetized and then transcardially perfused with 2-5 ml prewarmed HBSS (Hank's Balanced Salt Solution; Lonza), followed by perfusion with 4\% PFA in PBS until the tissue was fixed using an automated pump (Heidolph, pump drive 5001). Then, brains were isolated and postfixed overnight at $4^{\circ} \mathrm{C}$ in a $4 \%$ PFA solution. Subsequently, brains were dehydrated, embedded in paraffin (MICROM HMP 110; MICROM AP 280) and cut with microtome (MICROM HM 400) into $5 \mu \mathrm{m}$ thick sections.

\subsubsection{DAB staining of paraffin sections}

Mouse brain sections were deparaffinized in xylenes using two changes in xylene and one in xylene/isopropanol for $10 \mathrm{~min}$, followed by gradual hydration of the sections through graded alcohols: 5 min washes in 100\%-, 90\%-, 70\%-, 50\% ethanol to $\mathrm{H}_{2} \mathrm{O}$. Antigen unmasking was performed by heat treatment in sodium citrate buffer for $10 \mathrm{~min}$. and cooled for $20 \mathrm{~min}$ in the same buffer. Slides were washed for $5 \mathrm{~min}$ in Tris-M buffer and the endogenous peroxidase activity was blocked by incubation in $3 \% \mathrm{H}_{2} \mathrm{O}_{2}$ for 5 min. After one wash with Tris-M buffer, sections were incubated in the IHC blocking solution for $10 \mathrm{~min}$ at RT and subsequently incubated with the primary antibody diluted in the blocking solution overnight at $4^{\circ} \mathrm{C}$. Sections were washed in Tris-M buffer and incubated with the biotinylated secondary (Zytomed) antibody for $10 \mathrm{~min}$ at RT. After washing with Tris-M buffer, sections were incubated with the streptavidin enzyme complex (Zytomed) 
for 10 min at RT, which is linked to the primary antibody through the secondary antibody. Then, sections were washed in Tris buffer and incubated with the DAB (3,3'diaminobenzidine) (DAKO) solution for $10 \mathrm{~min}$, followed by two washes with $\mathrm{H}_{2} \mathrm{O}$ and staining of the nuclei with haematoxylin. Finally, sections were dehydrated and mounted with Eukitt (Kindler GmbH).

\subsubsection{Immunocytochemistry of heterologous cells and primary neurons}

Heterologous cells or primary neurons were grown for the indicated times on polyornithine coated glass coverslips (Menzel-Gläser) in a 24 well plate. To fix the cells, the media was removed and cells were washed twice in PBS. Then, $0.5 \mathrm{ml}$ of $4 \%$ PFA diluted in PBS was added to the wells and incubated for $10 \mathrm{~min}$ at RT. After two washes with PBS, cells were permeabilized with $0.5 \mathrm{ml}$ of PBS containing $0.4 \%$ Triton-X 100 for $10 \mathrm{~min}$ at RT. Again, cells were washed twice with PBS. To reduce non-specific binding of the antibody, cells were incubated with blocking buffer for $30 \mathrm{~min}$ at RT. Primary antibody was diluted in blocking buffer and incubated for either $1 \mathrm{hr}$ at RT or overnight at $4^{\circ} \mathrm{C}$. Afterwards, coverslips were washed twice with blocking buffer and incubated with the secondary antibody diluted in blocking buffer for 30-45 min at RT. The secondary antibody is coupled to a fluorophore, which is either Cy2 or Cy3 (Cy2 ${ }^{\mathrm{TM}} / \mathrm{Cy}^{\mathrm{Tm}}$, affinity pure goat anti mouse/ rabbit, Jackson Immuno Research, West Grove, PA, USA). Then, cells were washed twice with PBS and staining of nuclei was performed using the DNA dye bisbenzimide (Hoechst 33258) for 5 min at RT. Cells were washed twice with PBS and coverslips were mounted upside down on microscope slides (Marienfeld) in mounting media.

\subsection{Qualitative analysis of gene expression}

\subsubsection{Isolation of RNA}

Rodent non-neural tissue such as lung, liver, heart, kidney and spleen or neural tissue like cerebellum, cortex and hippocampus were homogenized in $1 \mathrm{ml}$ Trizol reagent using a 2 ml Dounce. Then 1/5 volume (200 $\mu \mathrm{l})$ of chloroform was added and mixed by inversion of the tube, followed by incubation for $15 \mathrm{~min}$ on ice. The solution was separated into 3 three different phases by centrifugation at $13000 \mathrm{rpm}$ for $15 \mathrm{~min}$ at $4^{\circ} \mathrm{C}$. The upper aqueous phase, which contains the RNA, was transferred into a fresh tube and mixed with an equal volume of isopropanol. Samples were kept on ice for $15 \mathrm{~min}$ to allow the RNA to precipitate. Thereafter, samples were centrifuged at $13000 \mathrm{rpm}$ for $15 \mathrm{~min}$ at $4^{\circ} \mathrm{C}$. Pellets were washed in $70 \%$ 
ethanol prepared with autoclaved water and spun down at $13000 \mathrm{rpm}$ for $10 \mathrm{~min}$ at $4^{\circ} \mathrm{C}$. Pellets were air-dried and resuspended in $200 \mu \mathrm{l}$ autoclaved water. For second precipitation, $70 \mu \mathrm{l}$ of $3 \mathrm{M}$ ammoniumacetate $\left(\mathrm{NH}_{4} \mathrm{Ac}\right)$ and $500 \mu \mathrm{l} 100 \%$ ethanol p.a. were added and tubes were inverted several times. Precipitated RNA was spun down at $13000 \mathrm{rpm}$ for $20 \mathrm{~min}$ at $4^{\circ} \mathrm{C}$. Pellets were air-dried and dissolved in $100 \mu \mathrm{l}$ autoclaved water. RNA concentration was measured with a spectrophotometer (Amersham Biosciences, Ultro spec 3100 pro).

\subsection{2 cDNA synthesis from total RNA}

$2 \mu \mathrm{g}$ of total isolated RNA was oligo(dt)-primed and transcribed into cDNA with the SuperScript ${ }^{\mathrm{TM}}$ III First-Strand Synthesis for RT-PCR Kit (Invitrogen ${ }^{\mathrm{TM}}$ ) according to the manual.

\subsection{Expression plasmid construction and cloning}

\subsubsection{General DNA construct amplification}

All DNA constructs were amplified using 1.25 units of Pfu DNA polymerase and the corresponding buffer with 10 pmol forward primer, 10 pmol reverse primer and $10 \mathrm{mM}$ dNTPs (each) in a thermocycler. The optimal cycling parameters were adjusted according to the melting temperature of the primer pair and the amplicon size. The annealing temperature was set at $5^{\circ} \mathrm{C}$ below the melting temperature of the primer pair and the elongation time at $72^{\circ} \mathrm{C}$ was calculated according to the polymerase, 1 min for each 500 basepairs of DNA to be amplified. The cycles were repeated 30 times.

\begin{tabular}{l|c|l}
\hline \multicolumn{3}{c}{ PCR Program } \\
\cline { 1 - 2 } 1. Denaturation $95^{\circ} \mathrm{C}$ & $3 \mathrm{~min}$ & \\
\cline { 1 - 2 } 2. Denaturation $95^{\circ} \mathrm{C}$ & $30 \mathrm{sec}$ & \multirow{2}{*}{$\rightarrow$ go to step 2 for } \\
\cline { 1 - 2 } 3. Annealing ${ }^{\circ} \mathrm{C}$ & $30 \mathrm{sec}$ & anor $29 \mathrm{cycles}$ \\
\cline { 1 - 2 } 4. Elongation $72^{\circ} \mathrm{C}$ & $\mathrm{x} \mathrm{min}$ & \\
\cline { 1 - 2 } 5. Final elongation $72^{\circ} \mathrm{C}$ & $5 \mathrm{~min}$ &
\end{tabular}

The size and the quality of the PCR product was determined with agarose gel electrophoresis. As marker a $1 \mathrm{~kb}$ or $100 \mathrm{bp}$ ladder was used depending on the size of the PCR product. PCR fragments were cut out and extracted using the Nucleo Spin ${ }^{\circledR}$ Extract II Kit following the manual. 


\subsubsection{Cloning}

Cleaned PCR products and the respective target expression vectors were digested in a minimal volume of $50 \mu \mathrm{l}$ with the corresponding restriction enzymes for $1-2 \mathrm{hr}$ at $37^{\circ} \mathrm{C}$. Expression vectors were also digested subsequently with alkaline phosphatase, which catalyzes the removal of $5^{\prime}$ phosphate groups of DNA to prevent self-ligation. PCR products were directly purified using Nucleo Spin ${ }^{\circledR}$ Extract II Kit, whereas expression vectors were analyzed with agarose gel electrophoresis on a 1\% agarose gel to confirm efficient linearization. Only linearized vector was cut out and cleaned with the Nucleo Spin ${ }^{\circledR}$ Extract II kit. To ligate $16 \mu \mathrm{l}$ of digested PCR product, $1 \mu \mathrm{l}$ of digested target expression vector, $2 \mu \mathrm{l}$ of ligation buffer and $1 \mu \mathrm{l} \mathrm{T} 4$ DNA ligase were mixed together and ligated either $1 \mathrm{hr}$ at RT or overnight at $4^{\circ} \mathrm{C}$. Entire ligation reaction was transformed into $100 \mu$ l of chemocompetent DH5 $\alpha$ E. coli cells, incubated on ice for $20 \mathrm{~min}$, heat shocked at $42^{\circ} \mathrm{C}$ for $1 \mathrm{~min}$ and then replaced on ice for $1 \mathrm{~min}$. Afterwards, $1 \mathrm{ml}$ of prewarmed 2xYT media without antibiotics was added and cells were incubated for $1 \mathrm{hr}$ at $37^{\circ} \mathrm{C}$. Cells were spun down at $4000 \mathrm{rpm}$ for $3 \mathrm{~min}$ and plated on a $2 \mathrm{xYT}$ agar plate with $50 \mu \mathrm{g} / \mathrm{ml}$ ampicilline or kanamycin depending on the resistance gene carried by the vector for selection. Plates were incubated overnight at $37^{\circ} \mathrm{C}$.

For verification of positives clones, colonies were picked with an autoclaved pipett tip and transferred into $2 \mathrm{ml}$ of 2xYT media with the appropriate antibiotics. These minicultures were incubated overnight at $37^{\circ} \mathrm{C}$. Then, plasmid DNA was isolated with an alkaline lysis protocol and subjected to restriction digest to detect successful ligation. The Nucleo Spin ${ }^{\circledR}$ Plasmid Quick Pure was used to purify DNA from confirmed positive clones followed by sequencing analysis by the AGCTLab at the MPI of Experimental Medicine. For alkaline lysis, cell suspension was transferred into a $2 \mathrm{ml}$ tube and spun down for $3 \mathrm{~min}$ at $13000 \mathrm{rpm}$. Pellet was resuspended in $200 \mu \mathrm{l}$ of GTE buffer with freshly added RNase (5 $\mu$ l of $10 \mathrm{mg} / \mu \mathrm{l}$ stock solution) and incubated at RT for $5 \mathrm{~min} .400 \mu \mathrm{l}$ of freshly prepared bacteria lysis buffer was added and mixed gently by inverting the tube. Sample was placed on ice for $5 \mathrm{~min}$. Then, $300 \mu \mathrm{l}$ of ice cold neutralization buffer (3M potassium acetate) was added, mixed gently and incubated on ice for $5 \mathrm{~min}$. Sample was centrifuged for $5 \mathrm{~min}$ at $13000 \mathrm{rpm}$. Supernatant was transferred into a new tube and $540 \mu \mathrm{l}$ of isopropanol was added. By mixing the two solutions the DNA was precipitated. Sample was incubated for 15 min at RT and then centrifuged at max speed for 2 min. Pellet was washed once with $70 \%$ ethanol, air-dried and resuspended in $50 \mu \mathrm{l}$ of $\mathrm{H}_{2} \mathrm{O} .5 \mu \mathrm{l}$ of DNA was used for restriction 
digestion with appropriate enzymes and analyzed by gel electrophoresis on a 1\% agarose gel.

For transfection of plasmid DNA into heterologous cells or primary neurons, plasmid DNA was amplified in chemo-competent DH5 $\alpha$ E. coli cells in $50 \mathrm{ml}$ or larger volumes of $2 \mathrm{xYT}$ medium with the appropriate antibiotics overnight in a $37^{\circ} \mathrm{C}$ shaker. Plasmid DNA was purified with the Nucleo Bond ${ }^{\circledR}$ Xtra Midi EF kit according to manufacturer's instructions.

\subsubsection{Site-directed mutagenesis of plasmids}

Site-directed mutagenesis was used to mutate the RNAi targeting sequence in pCMV-myc-RNF157 and pCMV-myc-Fe65 that renders these encoded molecules resistant to RNAi-mediated degradation. The primer pairs 22180/ 22181 with 6 silent mutations for RNF157 and 23636/23637 with 4 silent mutation for Fe65 in the sequence were designed according to the stratagene/Agilent technology manual using the provided formula:

$$
\mathrm{T}_{\mathrm{m}}=81.5+0.41(\% \mathrm{GC})(675 / \mathrm{N}) \% \text { mismatch }
$$

$\mathrm{N}$ is the primer length in bases and values for \%GC and \%mismatch are integral numbers. The protocol was then modified in the laboratory. 15 pmol primers were used in a PCR reaction using the Pfu DNA polymerase and the respective vector as template (10 ng). The following cycling parameters were used:

\begin{tabular}{c|c|l}
\hline \multicolumn{3}{c}{ Site-directed mutagenesis PCR } \\
\cline { 1 - 2 } Denaturation & $3 \mathrm{~min}$ & \\
\hline Denaturation & $30 \mathrm{sec}$ & \multirow{2}{*}{17 cycles } \\
\cline { 1 - 2 } Annealing $\left(55^{\circ}\right)$ & $30 \mathrm{sec}$ & \\
\hline Elongation & $1 \mathrm{~min} / 500 \mathrm{bp}$ & \\
\hline Final Elongation & $5 \mathrm{~min}$ &
\end{tabular}

Sample was then incubated on ice for $2 \mathrm{~min}$, digested with DpnI for $1 \mathrm{hr}$ at $37^{\circ} \mathrm{C}$ and chilled on ice for $10 \mathrm{~min} .1 \mu \mathrm{l}$ of the digested PCR product was transformed into chemo-competent DH5 $\alpha$ E. coli cells, plated on a 2xYT agar plate containing the appropriate antibiotics and incubated overnight at $37^{\circ} \mathrm{C}$. Successful site-directed mutagenesis was confirmed by sequencing. 


\subsection{4 shRNA design and plasmid construction}

The RNAi technology used in this study is based on the expression of short hairpin RNAs from a DNA template under the control of the RNA polymerase III U6 promoter. The oligonucleotide primers were designed manually in accordance with the following rules. The 5' sense primer should start either with AA, AG or GG followed by 18 nucleotides of the target DNA sequence and should have a GC content of $45-60 \%$. This sequence has to be specific for the target gene, should show no homologies to any other genes and contain not more than three adenosines in a row. Additionally, the targeting region should be identical between mouse, rat and human. The target sequence is followed by a modified Brummelkamp loop (TTCAAGCTT, with a unique HpaI restriction site) and the antiparallel target sequence with addition of CTTTTG (= Stop signal for the RNA polymerase III). The 3' antisense primer is complementary to the sense primer with addition of TTAA at the end. 2 $\mu \mathrm{l}$ of each primer $(50 \mathrm{pmol} / \mu \mathrm{l})$ was resuspended in $46 \mu \mathrm{l}$ of annealing buffer, incubated for $4 \mathrm{~min}$ at $95^{\circ} \mathrm{C}, 10 \mathrm{~min}$ at $72^{\circ} \mathrm{C}$ and slowly cooled down $\left(0.1^{\circ} \mathrm{C} / \mathrm{sec}\right)$ to RT. Then, $20 \mu \mathrm{l}$ of the annealed oligonucleotides were phosphorylated in a kinase reaction for $30 \mathrm{~min}$ at $37^{\circ} \mathrm{C}$. In parallel to primer preparation, the pBluescript-U6 vector was digested with ApaI, blunted with T4 DNA polymerase, digested with EcoRI and dephosphorylated with calf intestinal phosphatase (CIP). Finally, 3-5 $\mu \mathrm{l}$ of phosphorylated oligonucleotides and $1 \mu \mathrm{l}$ of the pBluescript-U6 vector were used for ligation overnight at $4{ }^{\circ} \mathrm{C}$ and transformed into chemocompetent DH5 $\alpha$ E. coli cells. Verification of positive clones was done by digestion with HpaI and analyzed by gel electrophoresis on a $1 \%$ agarose gel.

\subsection{Culture of primary neurons or HEK 293T cells}

In this study, all cell culture work was performed under cell culture sterility conditions in a safety cabinet (Hera safe, Kendro). Cells were grown in a $\mathrm{CO}_{2}$-incubator (Hera cell $150, \mathrm{Kendro}$ ) at $37^{\circ} \mathrm{C}$ and $5 \% \mathrm{CO}_{2}$. Primary cells as well as heterologous cells were grown on $10 \mathrm{~cm}$ culture dishes, 6 or 24 well plates depending on the experimental requirements.

\subsubsection{Culture of primary cerebellar granule neurons}

Primary cerebellar granule neurons were prepared from isolated cerebella of postnatal day (P6) wistar rats. During isolation, cerebella were kept in HHGN medium on ice. Afterwards, the meninges of the cerebella were carefully removed under a dissection 
microscope. All following steps were performed under sterile conditions in a safety cabinet. Cerebella were transferred into a $50 \mathrm{ml}$ falcon and washed three times with HHGN medium. After all media was removed, $5 \mathrm{ml}$ of TDn was added and incubated $10 \mathrm{~min}$ at $37^{\circ} \mathrm{C}$ followed by $10 \mathrm{~min}$ at RT. Then, after three washes with HHGN medium, $4 \mathrm{ml}$ of DnB was added. The cerebella were triturated to a homogenous suspension and placed on ice for $5 \mathrm{~min}$. The supernatant was transferred into a $50 \mathrm{ml}$ falcon. The remaining tissue suspension was triturated again with DnB to a homogenous suspension. Both suspensions were combined and centrifuged for $5 \mathrm{~min}$ at $800 \mathrm{rpm}$. The pellet was resuspended in a final volume of $15 \mathrm{ml} \mathrm{Cbc}$ medium. Cells were counted in a hemocytometer. Before plating the

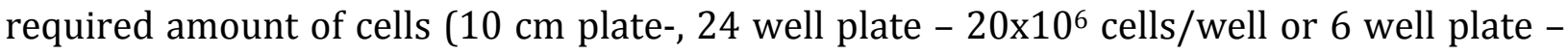
$5 \times 10^{6}$ cells/well), culture dishes (with or without coverslips) were incubated at least 30 min with poly-ornithine at $37{ }^{\circ} \mathrm{C}$ and washed twice with $\mathrm{H}_{2} 0$. At day in vitro 1 (DIV), neurons were treated with the anti-mitotic agent nucleoside cytosine arabinoside (AraC, 10 $\mathrm{mM}$ ) to prevent the proliferation of mitotic non-neuronal cells. Furthermore, cultures were fed with $35 \mathrm{mM}$ glucose at DIV 3 and 5.

\subsubsection{Culture of primary hippocampal neurons}

Primary hippocampal neurons were prepared from isolated hippocampi of E18 wistar rat embryos. During isolation, hippocampi were kept in 1xHBSS medium (BME [-] calcium [-] magnesium) on ice. The following steps were performed under sterile conditions in a safety cabinet. Hippocampi were washed three times with 1xHBSS. After reducing the media to $1.8 \mathrm{ml}, 300 \mu \mathrm{l}$ 1xTrypsin was added to the tissue and incubated for $10 \mathrm{~min}$ at $37^{\circ} \mathrm{C}$. Then, hippocampi were washed three times with $1 \mathrm{xHBSS}$ and triturated in $1 \mathrm{ml}$ hippocampal plating media to a homogenous suspension with freshly added DNase (2 $\mu \mathrm{g} / \mu \mathrm{l})$. Suspension was centrifuged at $4^{\circ} \mathrm{C}$ for $5 \mathrm{~min}$ at $800 \mathrm{rpm}$. Pellet was resuspended in $5 \mathrm{ml}$ plating media and cells were counted in a hemocytometer. Before plating the required amount of cells ( 24 well plate $-60 \times 10^{5}$ cells/well for transfection or $10 \times 10^{5}$ cells/well for immunocytochemistry or 6 well plate $-3 \times 10^{6}$ cells/well), culture dishes (with or without coverslips) were coated with poly-ornithine diluted in PBS for at least $30 \mathrm{~min}$ at $37{ }^{\circ} \mathrm{C}$ and washed twice with $\mathrm{H}_{2}$. At DIV 1, plating media was exchanged to hippocampal growth media. 


\subsubsection{Culture of HEK 293T cells}

Cell culture work was performed in a safety cabinet. HEK 293T cells were grown in HEK 293T media. For normal maintenance of HEK 293T cells, cells were grown in a $10 \mathrm{~cm}$ dish. Cells were split when the plate was 90\% confluent. After removal of the media, the plate was washed once with PBS and $1 \mathrm{ml}$ of 1xTrypsin was added onto the $10 \mathrm{~cm}$ culture dish. After $\sim 1 \mathrm{~min}$ incubation at $37^{\circ} \mathrm{C}$ in the $\mathrm{CO}_{2}$-Incubator, adherent cells were removed from the plate by adding $9 \mathrm{ml}$ of medium and subsequent pipetting up and down. The homogenous trypsin-cell suspension was centrifuged at $4^{\circ} \mathrm{C}$ for $5 \mathrm{~min}$ at $800 \mathrm{rpm}$. The supernatant was discarded and cells were resuspended in $5 \mathrm{ml}$ medium. Cell suspension was placed either into 6 well or $10 \mathrm{~cm}$ plates with preincubated medium and distributed by shaking. For a $10 \mathrm{~cm}$ plate $1 \mathrm{ml}$ and for a 6 well plate $180 \mu \mathrm{l}$ of the cell suspension was used.

\subsection{Transfection of primary neurons and HEK 293T cells}

The introduction of plasmid DNA into neurons or HEK 293T cells was done using a calcium phosphate transfection method. For both protocols the same 2xHBSS buffer was used.

\subsubsection{Transfection of primary neurons}

Primary cerebellar granule neurons (CGNs) were transfected at the indicated time points. The conditioned culture media was collected and incubated in $37^{\circ}$ incubator. CGNs were washed twice with prewarmed DMEM (+ 4,5 g/l glucose [-] glutamine [-] pyruvate) and starved in a $5 \% \mathrm{CO}_{2}$ incubator with $0.5 \mathrm{ml}$ (per well of a 24 well plate) fresh DMEM (+ 4,5 g/l glucose [-] glutamine [-] pyruvate). During this time, the DNA/calcium phosphate precipitate was prepared. The DNA was diluted in $18 \mu \mathrm{l}$ of sterile water and first mixed with $2 \mu \mathrm{l}$ of 2,5 $\mathrm{mM} \mathrm{CaCl}_{2}$, then with $20 \mu \mathrm{l}$ of $2 x H B S S$. The precipitate was allowed to form complexes for $10 \mathrm{~min}$ at RT. $40 \mu \mathrm{l}$ of the mixture was added drop-wise to the culture, mixed gently and incubated at $37^{\circ} \mathrm{C}$ for $5-18$ min depending on the experimental design. Neurons were then washed twice with DMEM (+ 4,5 g/l glucose [-] glutamine [-] pyruvate) and the aforementioned conditioned media was added.

\subsubsection{Transfection of HEK 293T}

One day prior to transfection, confluent HEK 293T cells were split from a $10 \mathrm{~cm}$ plate into a 6 well plate format. Since the uptake of the precipitated DNA is related to the 
presented surface area of the cell to the media, cells were split in a 1:30 manner. The following volumes were adjusted for one well of a 6 well plate. $10 \mathrm{ng}$ - $2 \mu \mathrm{g}$ plasmid DNA was diluted in $90 \mu \mathrm{l}$ sterile water. For precipitation, DNA solution was mixed with $10 \mu \mathrm{l}$ 2,5 $\mathrm{M} \mathrm{CaCl}_{2}$ followed by the addition of $100 \mu \mathrm{l} 2 \mathrm{xHBSS}$ and mixed gently. DNA solution was incubated for at least $10 \mathrm{~min}$ at RT and then added drop-wise to the cells. Transfected HEK 293T cells were kept in culture either three days for co-immunoprecipitation analysis and four days for validation of vector-based RNAis.

\subsection{Analysis of neuronal morphology}

Neurons were prepared as described in 2.13.1 or 2.13.2 and transfected at DIV 4 using the calcium phosphate transfection method. For loss-and gain-of-function experiments, neurons were cultured in a 24 well plate and transfected with the respective expression plasmids together with an expression plasmid for GFP $(0.3 \mu \mathrm{g})$ and the antiapoptotic protein Bcl- $\mathrm{x}_{\mathrm{L}}(0.3 \mu \mathrm{g})$ to avoid any effects of these manipulations on neuronal survival. The calcium phosphate precipitate was added drop-wise on granule neurons starved for $45 \mathrm{~min}$ for $15 \mathrm{~min}$. After fixation with 4\% PFA at the appropriate time points, neurons were subjected to immunocytochemistry using a polyclonal GFP antibody. Analysis of axonal and dendritic morphology was carried out by capturing at least 30 random pictures of GFP-positive neurons in a blinded manner using a fluorescence microscope at 20x magnification. Axonal and dendritic length was measured from the same granule neuron using ImageJ 1.410 software (http://rsb.info.nih.gov/ii).

\subsection{Survival assays in neurons}

Survival assays were performed for the most parts as described (Becker \& Bonni, 2004). Neurons were prepared as described in 2.13.1 or 2.13.2 and cultured in a 24 well plate. Neurons were transfected at DIV 2 with the respective expression plasmids together with a plasmid encoding ß-galactosidase using the calcium phosphate transfection method. The calcium phosphate precipitate was added drop-wise to the granule neurons starved for $30 \mathrm{~min}$ for $3 \mathrm{~min}$. For loss-and gain-of-function experiments, neurons were cultured for six days. After fixation with 8\% PFA, which was directly applied into the culture medium, neurons were subjected to immunocytochemistry using the monoclonal ß-galactosidase antibody and staining of the nuclei was performed using the DNA dye bisbenzimide (Hoechst 33258). Neuronal apoptosis was assessed in ß-galactosidase expressing neurons 
based on the integrity of neurites and the morphology of the nucleus. Analyses were done in a blinded manner ( $\mathrm{n} \geq 100$ cells per condition) using a fluorescence microscope.

\subsection{Yeast Two-Hybrid}

The Yeast Two-Hybrid screen was used to identify interacting proteins of RNF157. The principle of this method is a bait protein fused to the Gal4 DNA-binding domain (DNABD) while the cDNA library of fusion proteins is fused to the Gal4 DNA-activation domain (DNA-AD). When bait and prey are interacting, the Gal4 DNA-BD and AD are brought into close proximity. The Gal4 DNA-BD is then able to bind to the UAS (Upstream activating sequence) of the Gal4 promoter while the Gal4 DNA-AD can initiate transcription of downstream reporter genes.

\subsubsection{Media, buffer and stock solutions used for Yeast Two-Hybrid screen}

All media were autoclaved. For culture plates, 2\% of agar was added to the media before autoclaving. All buffers were either autoclaved or sterile filtered.

\section{YPD medium (1L)}

20 g peptone, 10 yeast extract - filled up with $\mathrm{ddH}_{2} \mathrm{O}$ to $950 \mathrm{ml}$; adjusted to $\mathrm{pH}$ 5.8. After autoclaving and cooling, $50 \mathrm{ml}$ of a $40 \%$ glucose solution was added.

\section{SD medium (synthetic dropout medium) (1L)}

$6.7 \mathrm{~g}$ yeast nitrogen base without amino acids, $\mathrm{X} g$ dropout supplement (Clontech Laboratories, Inc. $(X=0.69 \mathrm{~g}$ for [-] Leu; 0.64g for [-]Leu [-]Trp; 0.62 for [-]Leu [-]Trp []His) filled up with ddH20 to $950 \mathrm{ml}$; adjust to $\mathrm{pH}$ 5.8. After autoclaving and cooling, $50 \mathrm{ml}$ of a $40 \%$ glucose solution was added. For SD plates $50 \mathrm{mM}$ 3-amino-1,2,4-triazole (3-AT) was applied.

\section{Buffers and reagents:}

10x TE: 0.1 M Tris-HCl, 10 mM EDTA, pH 7.5

1 M Lithium acetate, pH 7.5 was adjusted with acetic acid $50 \%$ Polyethylene glycol (PEG)-4000 solution 
Z-buffer (1L): $16.1 \mathrm{~g} \mathrm{Na}_{2} \mathrm{HPO}_{4} \times 7 \mathrm{H}_{2} 0,5.5 \mathrm{~g} \mathrm{NaH} \mathrm{PO}_{4} \times 7 \mathrm{H}_{2} 0,0.75 \mathrm{~g} \mathrm{KCl}, 0.246 \mathrm{ggSO}_{4} \mathrm{x}$ $7 \mathrm{H}_{2} \mathrm{O}$ with $\mathrm{pH} 7.0$

X-gal stock: $20 \mathrm{mg} / \mathrm{ml}$ diluted in Dimethlyformamid (DMF), stored at $-20^{\circ} \mathrm{C}$

\subsubsection{Generation of Gal4 DNA-BD/bait construct}

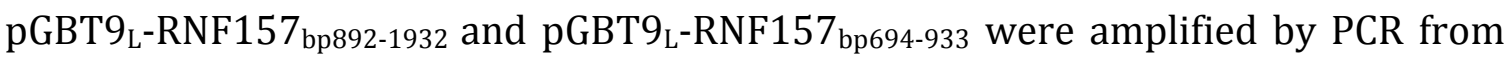
pCMV-myc-RNF157 with the primer pairs 16334/16335 and 17172/17257, respectively. The pGBT9 ${ }_{\mathrm{L}}$-RNF157 $7_{\mathrm{bp1}-921}$ was generated by J. Stegmüller using the primers 16420 and 16421. Constructs were confirmed by restriction digestion, gel electrophoresis and sequence analysis.

\subsubsection{Transformation of bait plasmid into yeast (small scale transformation)}

Transformation of the yeast strain CG-1945 was performed using a heat shock procedure. The CG-1945 yeast strain is auxotroph for tryptophan and leucin and harbors the lacZ and HIS3 reportergene under the control of the Gal4 promoter.

Stored yeast cells at $-80^{\circ} \mathrm{C}$ were plated on a YPD plate and inoculated at $30^{\circ} \mathrm{C}$. One single colony was grown at $150 \mathrm{rpm}$ in liquid YPD medium at $30^{\circ} \mathrm{C}$ for one or two days. About 5 $\mathrm{ml}$ of the culture was pelleted by centrifugation at $5000 \mathrm{rpm}$. The following reagents were added to the pellet in the same order and vortexed softly after each reagent: $20 \mu \mathrm{g}$ herring sperm DNA + $1 \mu \mathrm{g}$ „bait“ DNA, $500 \mu \mathrm{l}$ PLATE-mix (for $10 \mathrm{ml}: 8 \mathrm{ml}$ 50\% PEG-4000, $1 \mathrm{ml} 1 \mathrm{M}$ LiAc and $1 \mathrm{ml} \mathrm{10x} \mathrm{TE)} \mathrm{and} 20 \mu \mathrm{l}$ DTT. The transformation was incubated at RT for 6-8h without shaking. After $7 \mathrm{hr}$ the mixture was heat-shocked for $10 \mathrm{~min}$ at $42^{\circ} \mathrm{C}$ and chilled at RT for $5 \mathrm{~min}$ at RT. Yeast cells were harvested by centrifugation for $20 \mathrm{sec}$ at $2000 \mathrm{rpm}$,

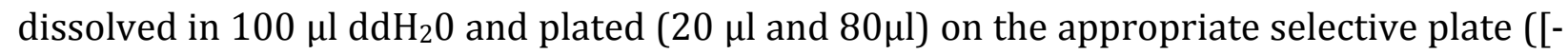
]Trp for analysis if yeast cells have incorporated the „bait" plasmid or on [-]His plates to determine auto-activation of the His-reportergene by the „bait“ plasmid). As negative control a transformation without „bait“ was performed. Yeast cells were grown for 2-3 days at $30^{\circ} \mathrm{C}$.

\subsubsection{Transformation of yeast cells with cDNA library (large scale transformation)}

The cDNA library used in this study was a postnatal mouse brain MATCHMAKER cDNA library (Lot: 6090287, Clontech). 
Yeast colonies containing the „bait" plasmid was inoculated overnight in $10 \mathrm{ml}$ of SD [-]Trp medium of which $5 \mathrm{ml}$ were grown in a second $100 \mathrm{ml}$ SD [-]Trp overnight culture. Yeast cells were pelleted by centrifugation for $3 \mathrm{~min}$ at $2500 \mathrm{rpm}$ and resuspended in 300 $\mathrm{ml}$ of prewarmed YPD medium (OD 0.2-0.3). Yeast cells were grown to OD of 0.7-0.8 and harvested by centrifugation for $4 \mathrm{~min}$ at $4500 \mathrm{rpm}$ at RT. Pellet was washed once in $40 \mathrm{ml}$ $\mathrm{ddH}_{2} \mathrm{O}$ and again pelleted. The following reagents were added to the pellet in the same order for transformation of the cDNA library: $14.4 \mathrm{ml} 50 \%$ PEG-4000, $2.16 \mathrm{ml} 1 \mathrm{M} \mathrm{LiAc}, 0.3$ $\mathrm{ml}$ herring sperm carrier DNA (10 mg/ml), $0.625 \mathrm{ml}$ plasmid library (250 $\mu \mathrm{g}$ ) and $0.825 \mathrm{ml}$ $\mathrm{ddH}_{2} \mathrm{O}$. The transformation was mixed gently and incubated at $200 \mathrm{rpm}$ for $30 \mathrm{~min}$ at $30^{\circ} \mathrm{C}$. Then, $2 \mathrm{ml}$ of DMSO was mixed gently and yeast cells were heat shocked for $30 \mathrm{~min}$ at $42^{\circ} \mathrm{C}$. Yeast cells were pelleted for $30 \mathrm{sec}$ at $3000 \mathrm{rpm}$, resuspended in $8 \mathrm{ml}$ of dd $\mathrm{H}_{2} \mathrm{O}$ and incubated at RT for $10 \mathrm{~min} .250 \mu \mathrm{l}$ of transformed yeast cells were plated per [-]His [-]Trp [-]Leu [+] $50 \mathrm{mM}$ 3-AT plates (15 cm diameter) and incubated for 5 days at $30^{\circ} \mathrm{C}$. Colonies with a diameter $\geq 2 \mathrm{~mm}$ were replica plated for analysis. To eliminate false-positive clones, a ß-galactosidase assay was performed with one of the replica plates three days later. A nitrocellulose membrane was placed on the agar plate for $1 \mathrm{~min}$, incubated in liquid nitrogen for $30 \mathrm{sec}$, thaw at RT and placed with colonies facing up on a whatman paper

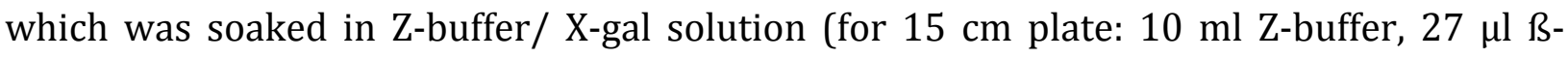
Mercaptoethanol, $167 \mu \mathrm{l}$-Gal stock) for $30 \mathrm{~min}-3 \mathrm{hr}$ depending on the color reaction. Positive indigo-blue colored colonies were selected for further analysis.

\subsubsection{Isolation of plasmid DNA from yeast}

Plasmid DNA of positive yeast clones was extracted from a $5 \mathrm{ml}$ overnight culture. Cells were pelleted in a $1.5 \mathrm{ml}$ tube by centrifugation and lysed together with acid-washed glass beads in $250 \mu \mathrm{l}$ Lysis buffer A1 (NuclepSpin ${ }^{\circledR}$ Plasmid QuickPure). Glass beads were spun down and isolation of plasmid DNA was performed according to the manual of NuclepSpin ${ }^{\circledR}$ Plasmid QuickPure Kit.

\subsubsection{Identification of interacting cDNA clones}

Isolated plasmid DNA from positive interacting yeast clones was analyzed by PCR. For amplification of the insert, $3 \mu \mathrm{l}$ DNA, $1 \mu \mathrm{l}$ of the pACT2 specific primers 1596 and 1681 and $25 \mu \mathrm{l}$ PCR SuperMix were used. The following cycling parameter were used: $95^{\circ} \mathrm{C}-5$ $\min , 95^{\circ} \mathrm{C}-30 \mathrm{sec}, 50^{\circ} \mathrm{C}-30 \mathrm{sec}, 72^{\circ} \mathrm{C}-5 \mathrm{~min}$ for 36 cycles and final elongation at $72^{\circ} \mathrm{C}-$ 
10 min. PCR reactions were analyzed by agarose gel electrophoresis. Yeast DNA samples, which produced a DNA fragment in PCR reactions, were send for sequencing analysis.

\subsection{Genotyping of RNF157 gene trap mice}

\subsubsection{Extraction of genomic DNA from mouse tails}

Tail biopsies incubated in $200 \mu \mathrm{l}$ of lysis buffer supplemented with $\mathrm{NaCl}$ and Proteinase $\mathrm{K}$ were incubated for $3 \mathrm{hr}$ or overnight at $55^{\circ} \mathrm{C}$. Samples were gently shaken and spun at $13000 \mathrm{rpm}$ for $10 \mathrm{~min}$ at RT. Supernatant was mixed with $0.5 \mathrm{ml} 100 \%$ ethanol p.a., followed by centrifugation at $13000 \mathrm{rpm}$ for $10 \mathrm{~min}$ at RT. The supernatant was discarded and the precipitated DNA was washed with 70\% ethanol. Then, the DNA was air-dried and dissolved in 50-100 $\mu \mathrm{l} \mathrm{H}_{2} 0$. Subsequently, $1 \mu \mathrm{l}$ of genomic DNA was used for PCR-based genotyping.

\subsubsection{RNF157 Genotyping PCR}

The PCR reactions for the RNF157 gene trap line were performed with the GoTaq ${ }^{\circledR}$ DNA polymerase according the manufacturer's protocol. The primer pair 25173/25172 was used to amplify the wild type allele, whereas the mutant allele was amplified using the primer pair $25171 / 25172$. The following PCR program was used to genotype the RNF157 gene trap mice:

\begin{tabular}{|c|c|c|c|}
\hline \multicolumn{4}{|c|}{ RNF157-PCR } \\
\hline Denaturation & $95^{\circ} \mathrm{C}$ & $3 \mathrm{~min}$ & \\
\hline Denaturation & $95^{\circ} \mathrm{C}$ & $30 \mathrm{sec}$ & \multirow{3}{*}{29 cycles } \\
\hline Annealing & $48^{\circ} \mathrm{C}$ & $30 \mathrm{sec}$ & \\
\hline Elongation & $72{ }^{\circ} \mathrm{C}$ & $25 \mathrm{sec}$ & \\
\hline Final Elongation & $72^{\circ} \mathrm{C}$ & $5 \mathrm{~min}$ & \\
\hline
\end{tabular}

\subsection{Statistics}

Statistical analysis was performed using GraphPad Prism 5.0c software. The data was analyzed by unpaired Student's t-test and one-way or two-way ANOVA (ANalysis Of VAriance). Errors are displayed as standard error of the mean (SEM). 


\section{Results}

\subsection{Characterization of the E3 ligase RNF157}

The ubiquitin-proteasome system (UPS) has emerged as a crucial regulator of brain development. Since E3 ligases are the most numerous components of the UPS, I sought to identify brain-dominant candidates to unravel their role in neurons. Using the information of the Cerebellar Development Transcriptome Database (CDT-DB), I found an E3 ligase with brain-dominant expression pattern: the RING finger protein RNF157. Aside from its striking expression, RNF157 shares a high level of similarity with the E3 ligase Mahogunin RING finger-1 (Mgrn1), which has been previously implicated in spongiform neurodegeneration (He et al, 2003). Owing to the fact that RNF157 is an uncharacterized E3 ligase, reagents were unavailable and the analysis of RNF157 function thus required the generation of tools.

\subsubsection{Cloning of rat RNF157 cDNA}

As an initial step to clone rat RNF157, I examined the cDNA sequence of mouse, rat and human RNF157 provided by online databases. Using Ensembl, I found that human RNF157 is located on chromosome 17: 74,138,534-74,236,390 and there are 3 isoforms, which differ in size. Mouse RNF157 is located on chromosome 11: 116,197,667$116,274,318$ and appears in four isoforms. Since the Ensembl project showed no data for rat RNF157, the mouse RNF157 sequence was used for blastn search in the nucleotide collection $(\mathrm{nr} / \mathrm{nt})$ database of the $\mathrm{BLAST}^{\circledR}$ program. Alignment of mouse, human and predicted rat RNF157 sequences revealed that RNF157 is highly conserved between the analyzed species, but they differ in their N-terminal part (Appendix 5.1). The predicted rat RNF157 sequence is 75 bp shorter than mouse or human RNF157. Consequently, the rat RNF157 protein is 25 aa shorter than mouse or human RNF157. However, the RING domain of RNF157 is conserved between species (Figure 6). 


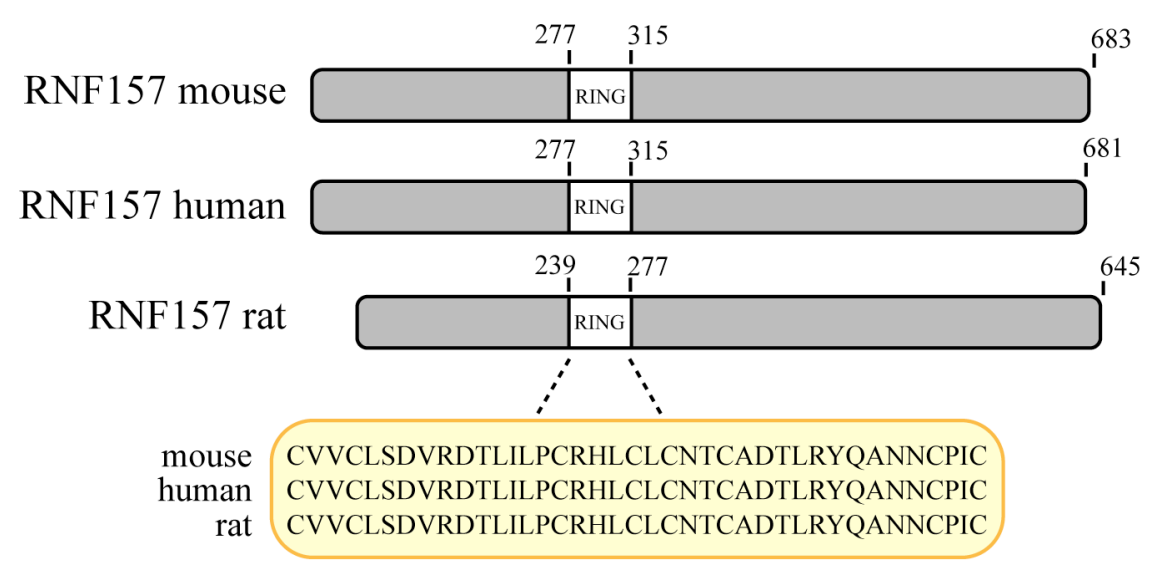

Figure 6. Protein sequence alignment for the RING domain of mouse, human and rat RNF157. The RING domain is evolutionary conserved between species.

Rat RNF157 was amplified in 2 fragments from brain tissue with the oligonucleotide primers $(14758 / 14759 ; 14760 / 14761)$ designed on the basis of the predicted rat RNF157 cDNA sequence. The PCR reaction with the first primer pair did not give any product. After further sequence analysis a second conserved in frame start-codon was found in all three analyzed RNF157 cDNA sequences (Appendix 5.1). Amplification of rat RNF157 with a sense-primer (14798) based on this sequence resulted in a PCR product. This shorter rat RNF157 sequence was used for all experiments described in my study.

\subsubsection{Expression and localization of RNF157 during brain development}

First, I established by RT-PCR which cell types of the brain express RNF157. cDNA from cerebellar granule neurons (days in vitro (DIV) 6, 8), the oligodendrocyte precursor cell line OLI-neu (DIV 2) and primary astrocytes in culture (DIV 2) revealed that RNF157 is present in all three cell types (Figure 7A).

To determine the spatial gene expression pattern of RNF157, I first analyzed the expression of RNF157 in different brain regions as well as in different organs of the body from wild type rat by RT-PCR (Figure 7B). RNF157 is highly expressed in cortex, cerebellum and hippocampus and detectable to a much lesser extent in lung, heart and kidney. No expression of RNF157 was detected in liver and spleen. Next, to analyze RNF157 expression at the protein level, I prepared crude lysates from the aforementioned tissues and examined those with immunoblotting using the RNF157 antibody. I found that RNF157 is expressed in cortex, cerebellum and hippocampus but not in lung, liver, heart, kidney and spleen (Figure 7C). 

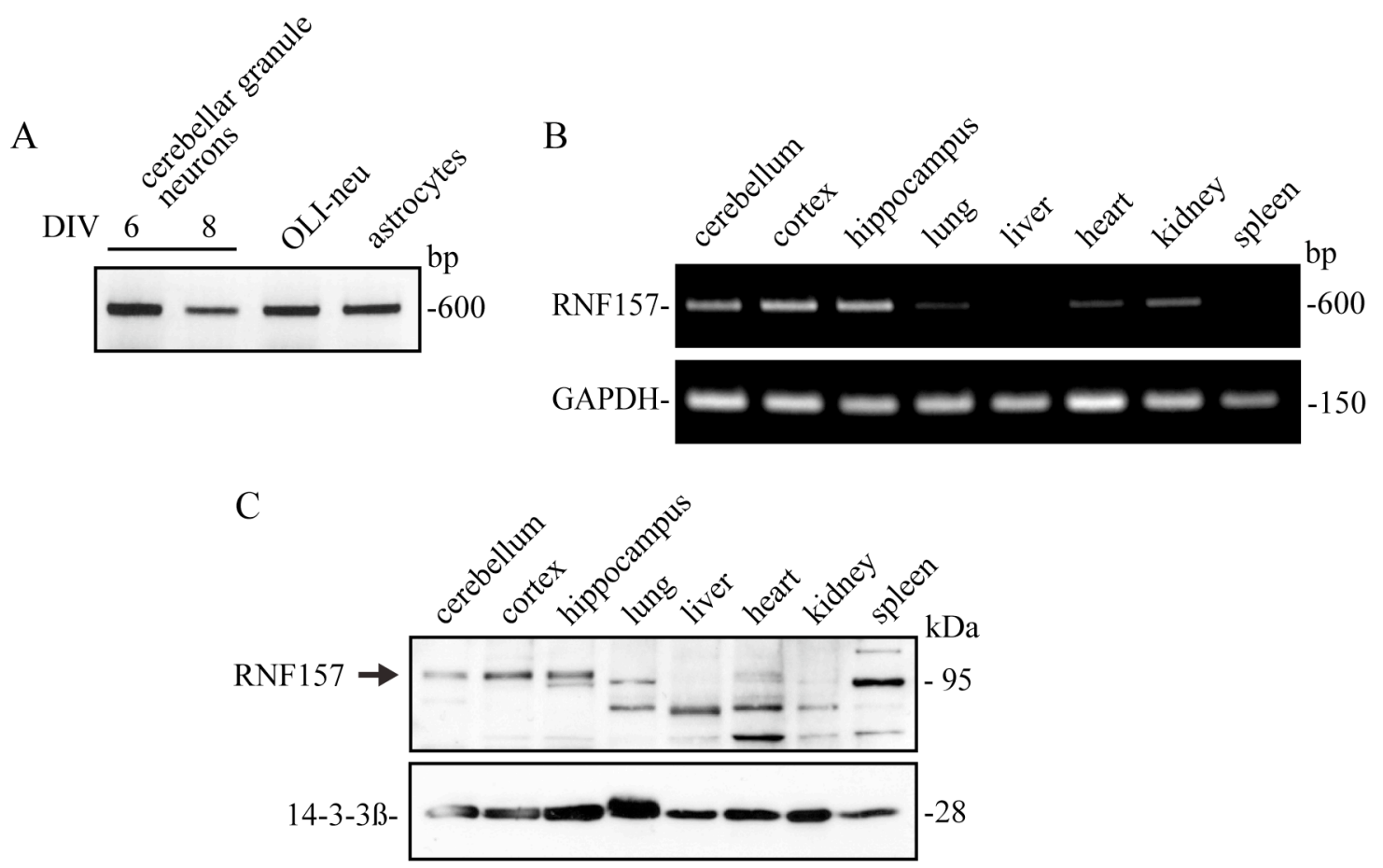

Figure 7. Brain-dominant expression of RNF157 in the brain. A) Reverse transcriptase polymerase chain reaction (RT-PCR) analysis showing the expression of RNF157 in rat cerebellar granule neurons (DIV 6/8), OLI-neu cells and primary astrocytes in vitro. B) RT-PCR analysis showing the expression of RNF157 in the indicated tissues collected from wild type postnatal day (P) 12 rat. GAPDH served as loading control. C) Indicated rat tissue lysates at P12 were subjected to immunoblotting using the RNF157 and 14-3-3ß antibodies. The latter served as loading control. Arrow indicates RNF157.

In further analysis, I examined together with my colleague Anna Holubowska the expression of RNF157 protein in cultured neurons as well as during brain development. RNF157 protein expression was examined either in rat cerebellar granule neurons prepared from postnatal day (P) 6 rat pups or in cortical neurons prepared from embryonic day (E) 18 rat embryos. Here, RNF157 is stably expressed in primary cerebellar and cortical neurons with increasing days in vitro (Figure 8A). In the developing brain however, we found that while RNF157 expression is postnatally upregulated in the cerebellum, it decreases from E18 to P60 in the cortex and is unchanged in the hippocampus (Figure 8B). Taken together, my data identifies RNF157 as a brain-dominant protein that is expressed throughout neuronal development. 
A
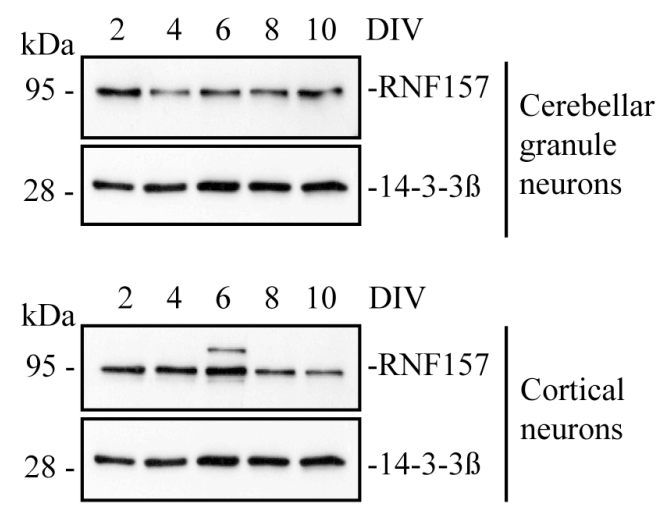

$\mathrm{B}$
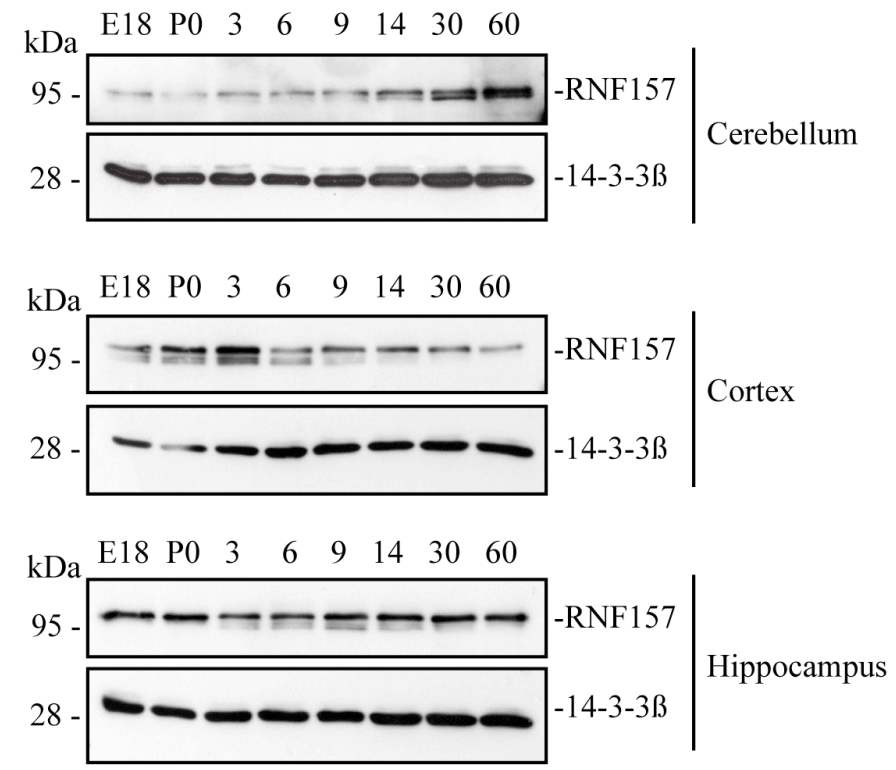

Figure 8. Developmental expression of RNF157 in the brain. A) Lysates of cerebellar granule and cortical neurons prepared from P6 or E18 rats respectively and placed in culture for the indicated days were immunoblotted using the RNF157 and 14-3-3ß antibodies. The latter served as loading control (Courtesy of A. Holubowska). B) Crude lysates of mouse cerebellum, cortex and hippocampus isolated at the indicated days were subjected to immunoblotting using the RNF157 antibody. 14-3-3ß served as loading control (Courtesy of A. Holubowska). E, embryonic; P, postnatal day.

\subsubsection{Subcellular localization of RNF157 in neurons}

To identify the subcellular localization of RNF157, I performed subcellular fractionation of cerebellar granule neurons at DIV 8. Cerebellar granule neuron lysate was sequentially separated into postnuclear supernatant (PNS) and nuclear fraction (NF). Equal amounts of protein from each fraction were subjected to Western blot analysis and probed with the RNF157, Sp1 or 14-3-3ß antibodies. The latter two served as markers for the nuclear fraction and postnuclear supernatant, respectively. I found that RNF157 localizes to the postnuclear supernatant in cerebellar granule neurons suggesting that RNF157 is a cytoplasmic protein (Figure 9).

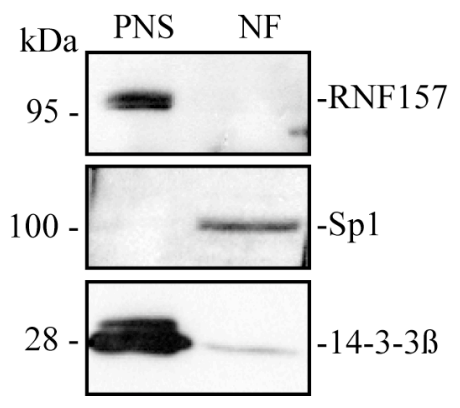

Figure 9. Cytoplasmic localization of RNF157 in neurons. Cerebellar granule neurons were subjected to subcellular fractionation. The nuclear fraction (NF) and the postnuclear supernatant (PNS) were immunoblotted with the RNF157, Sp1 or 14-3-3ß antibodies. The latter two served as positive controls for the NF and the PNS, respectively. 
In order to characterize the subcellular localization of endogenous RNF157 in neurons, cerebellar granule neurons and hippocampal neurons in culture were subjected to immunocytochemistry using the RNF157 antibody. Here, RNF157 localized to the cytoplasm and neurites in both neuronal cell types and was excluded from the nucleus (Figure 10). In polarized neurons, RNF157 was detectable in axons and dendrites (Figure 10B). Interestingly, RNF157 is restricted to primary dendrites (Figure 10B, lower panel).

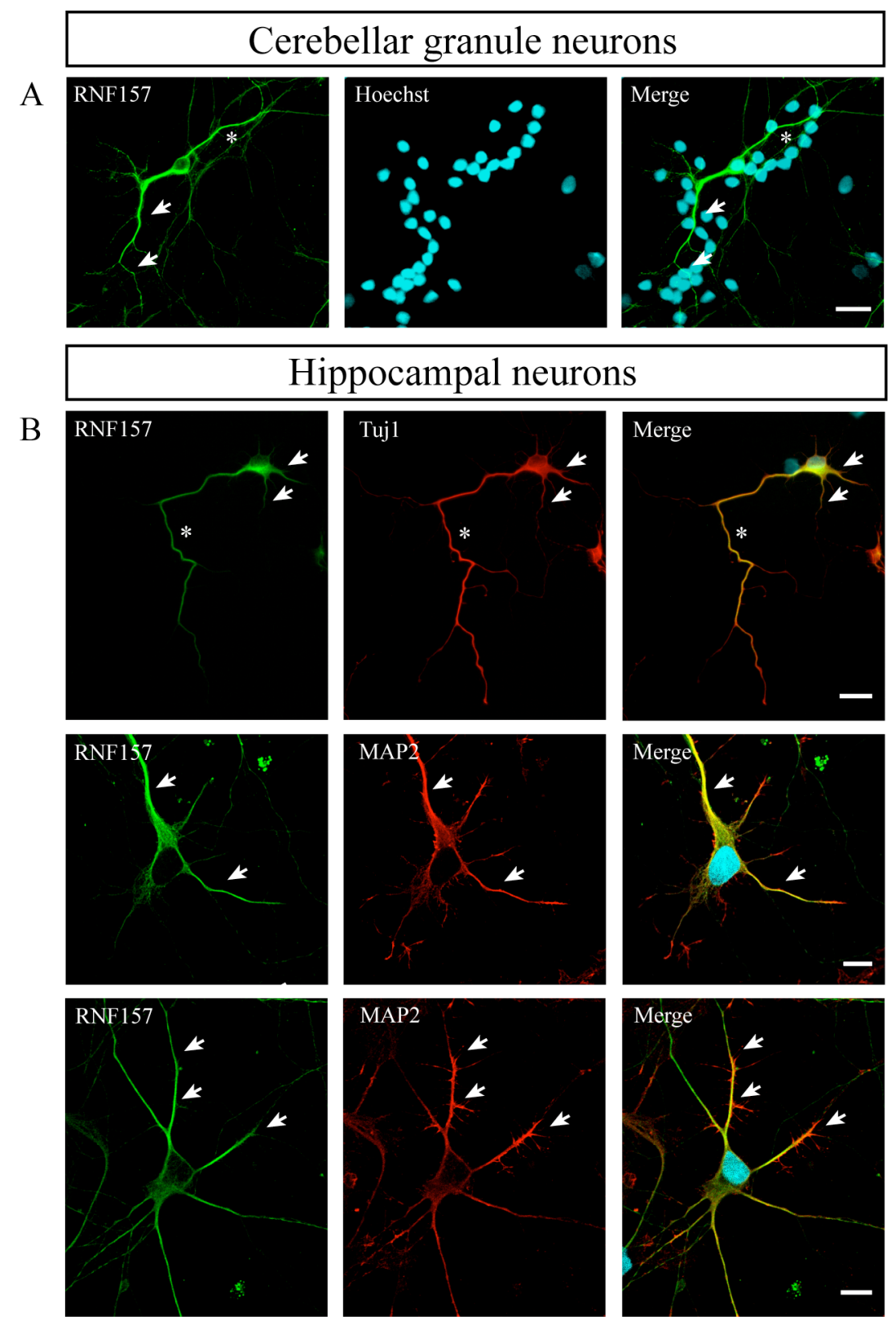

Figure 10. RNF157 localizes to the cytoplasm, axons and primary dendrites in neurons. A) Cerebellar granule neurons at DIV 7 were subjected to immunocytochemistry using the RNF157 antibody and the DNA dye bisbenzimide (Hoechst 33258). B) Hippocampal neurons at DIV 4 were subjected to immunocytochemistry using the RNF157, TujI or MAP2 antibodies and the DNA dye bisbenzimide (Hoechst 33258). Asterisks and arrows indicate axons and dendrites, respectively. Scale bar equals $20 \mu \mathrm{m}$. 
Finally, I asked whether RNF157 is present in dendritic spines. I examined the subcellular localization of RNF157 in hippocampal neurons at DIV 21. Neurons were immunostained with the RNF157 antibody and the PSD-95 antibody as a marker for dendritic spines. While I found that RNF157 is present in the cytoplasm, axons and dendrites of DIV 21 hippocampal neurons, RNF157 is absent from dendritic spines (Figure 11).

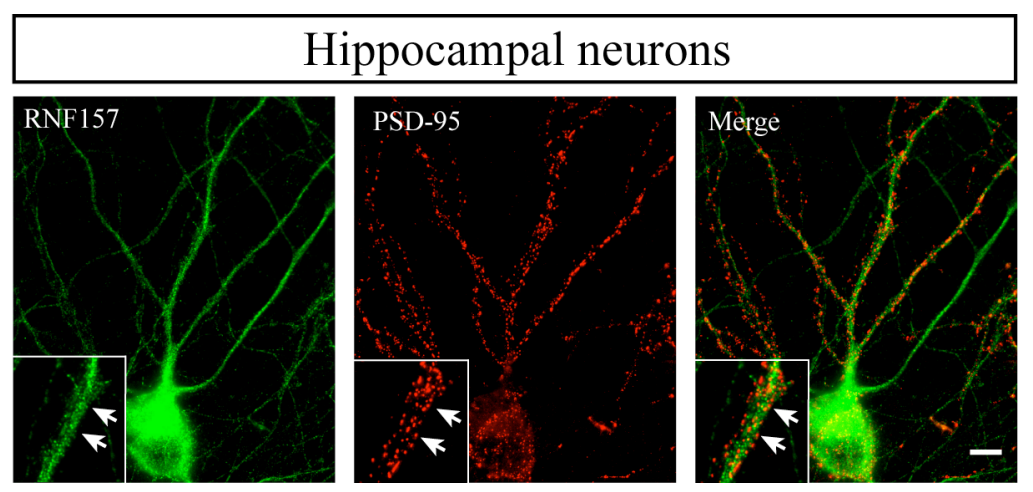

Figure 11. RNF157 is absent from dendritic spines. Hippocampal neurons at DIV 21 were subjected to immunocytochemistry using the RNF157 and PSD-95 antibodies. Nuclei were stained with the DNA dye bisbenzimide (Hoechst 33258). Arrows indicate spines. Scale bar equals $10 \mu \mathrm{m}$.

\subsection{Functional analyses of RNF157 in neurons}

Sequence and domain analyses revealed that RNF157 and Mgrn1 share a highly conserved N-terminus and an identical RING domain. In 2003, He and colleagues reported that Mgrn1 knockout mice develop severe age-dependent spongiform neurodegeneration (He et al, 2003). The role of RNF157 in the central nervous system however remains elusive. To shed light on the function of RNF157 in neurons, I took an RNA interference (RNAi) approach to carry out loss-of-function analysis using cerebellar granule as a model system.

\subsubsection{Validation of RNF157 shRNA constructs}

I started out by generating different vector-based RNAi plasmids targeting rat, mouse and human RNF157. To test the efficacy of the plasmids, I transfected HEK 293T cells with the control vector, RNF157 RNAi plasmids II or III together with a plasmid encoding myc-RNF157. After four days in culture, lysates of HEK 293T cells were subjected to immunoblotting with the myc antibody. These experiments revealed that expression of RNF157 RNAi II and III efficiently reduces the level of exogenous RNF157 in heterologous cells (Figure 12). 


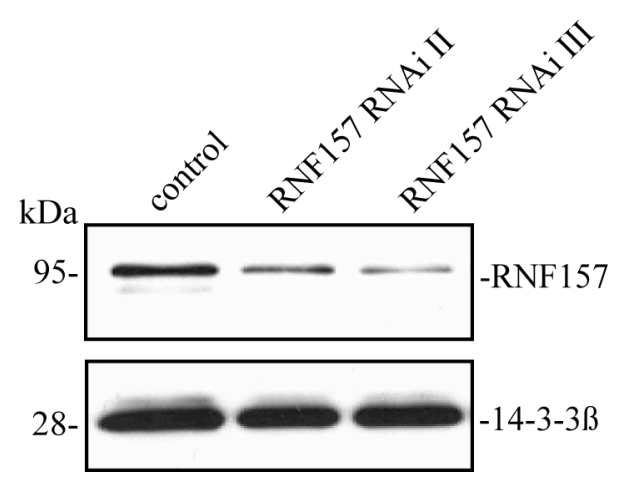

Figure 12. Efficient knockdown of exogenous RNF157 by RNF157 RNAi II and III. Lysates from HEK 293T cells transfected with the expression plasmid encoding myc-RNF157 together with the control vector, RNF157 RNAi plasmids II or III were immunoblotted with the myc antibody. 14-3-3ß served as loading control.

\subsubsection{RNF157 is a regulator of neuronal survival in cerebellar granule neurons}

To examine the role of RNF157 in neurons, I transfected cerebellar granule neurons at DIV 2 with the control vector, RNF157 RNAi plasmids II or III together with a plasmid encoding ß-galactosidase. Four days later, I examined neurons for any abnormal phenotype as compared to control-transfected neurons. Interestingly, I found a dramatic increase in cell death in RNF157 RNAi-transfected neurons. While RNF157 RNAi II triggers a slight but significant increase in cell death, RNF157 RNAi III leads to a striking increase in apoptosis in cerebellar granule neurons (Figure 13A), reflecting the knockdown efficiency determined before. In addition, RNF157 knockdown neurons display hallmarks of apoptosis including pyknotic nuclei, fragmented axons and cleaved caspase-3 immunoreactivity (Figure 13B). These results suggest that RNF157 promotes neuronal survival in vitro. (In all further knockdown experiments, I used the RNF157 RNAi III and refer to it as RNF157 RNAi unless stated otherwise)

A

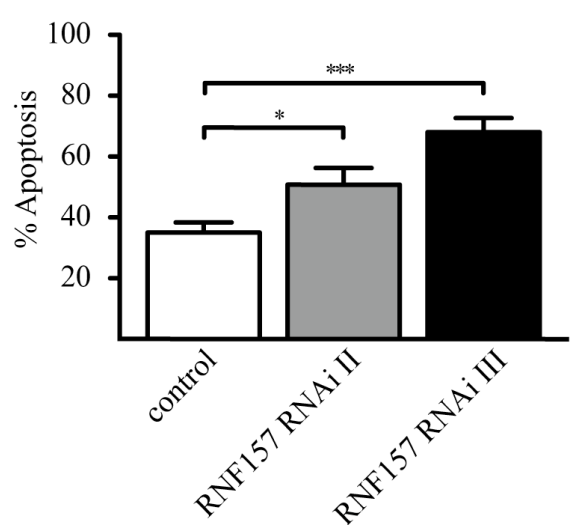

B

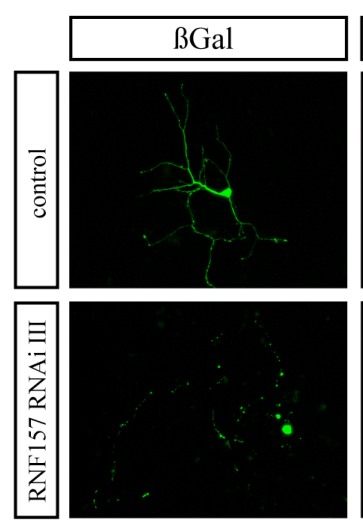

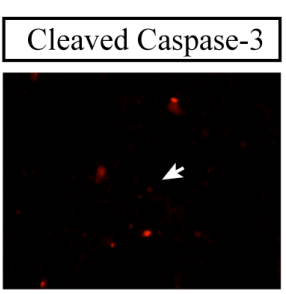

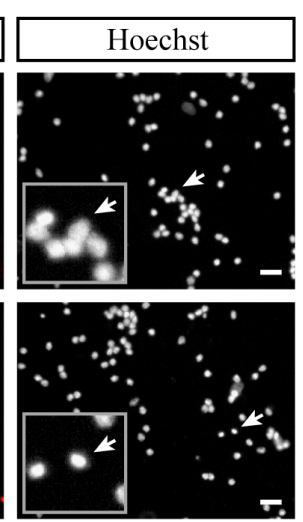

Figure 13. Knockdown of RNF157 increases neuronal apoptosis. A) Cerebellar granule neurons transfected at DIV 2 with the control vector, RNF157 RNAi plasmids II or III together with a plasmid encoding $ß$-galactosidase were subjected to immunocytochemistry using the ß-galactosidase ( $ß G$ al) antibody and the DNA dye bisbenzimide (Hoechst 33258) four days later. Percentage of apoptosis is presented as mean \pm SEM. Knockdown of RNF157 significantly increases apoptosis as compared to control-transfected neurons ( $\mathrm{n}=3$; ANOVA; $\left.{ }^{*} \mathrm{p}<0.05 ;{ }^{* *} \mathrm{p}<0.001\right)$. A total of 1225 neurons were analyzed. B) Cerebellar granule neurons 
transfected with the control vector or RNF157 RNAi plasmid III together with a plasmid encoding ßgalactosidase were subjected to immunocytochemistry at DIV 6 using the ß-galactosidase ( $B$ Gal) and cleaved caspase-3 antibodies and the DNA dye bisbenzimide (Hoechst 33258). Arrows indicate nuclei of transfected neurons in representative images. In the RNF157 knockdown condition, the nucleus is pyknotic and cleaved caspase-3 positive. Insets depict nuclei indicated with an arrow. Scale bar equals $20 \mu \mathrm{m}$.

Next, to demonstrate that the increase in apoptosis is the specific result of RNAiinduced knockdown of RNF157 and not an off-target effect, I performed rescue experiments. Thus, I generated an RNF157-Rescue (RNF157-Res) construct with 6 silent mutations in the RNF157 RNAi III targeting region to render it resistant to knockdown (Figure 14A). Immunoblotting of HEK 293T cell lysates transfected with the RNF157 RNAi plasmid together with wild type RNF157 or RNF157-Res expression plasmids revealed that the RNF157 RNAi efficiently reduces RNF157 wild type levels, but fails to knockdown RNF157-Res (Figure 14B). In subsequent experiments, cerebellar granule neurons were transfected with the control vectors, RNF157 RNAi plasmid with control vector, or both RNF157 RNAi plasmid with RNF157-Res expression plasmid together with a plasmid encoding ß-galactosidase. Here, I found that expression of RNF157-Res in the background of RNF157 knockdown significantly reduces apoptosis of cerebellar granule neurons (Figure 14C). These experiments indicate that the increase of apoptosis upon RNF157 RNAi is the result of a specific knockdown of RNF157. Together, my results identify RNF157 as a regulator of neuronal survival in cerebellar granule neurons in vitro.

A

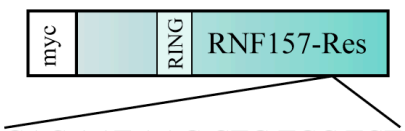

GAC AAT AAG CTG TGC TCT GAT AAC AAA CTC TGT TCG aa Asp Asn Lys Leu Cys Ser
B

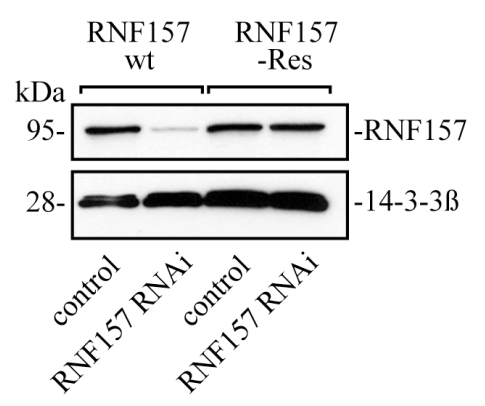

$\mathrm{C}$

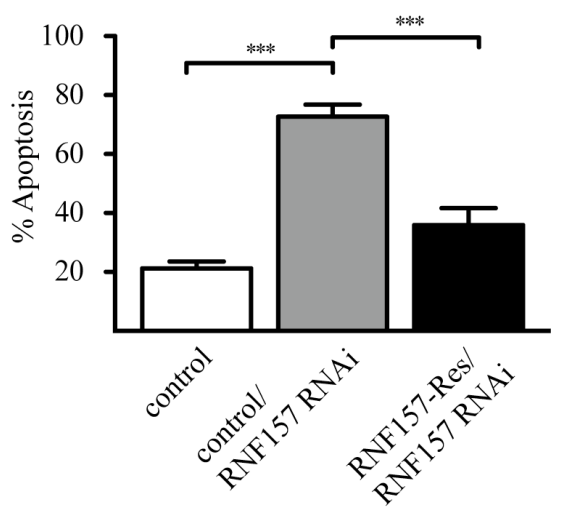

Figure 14. RNF157 is required for neuronal survival. A) Schematic representation of myc-RNF157-Res. Silent mutations that render RNF157-Res resistant to RNAi are indicated in red. B) Lysates of HEK 293T cells transfected with the control vector or RNF157 RNAi plasmid together with a plasmid encoding myc-RNF157 wild type or myc-RNF157-Res were immunoblotted using the myc or 14-3-3ß antibodies. The latter served as loading control. RNF157 RNAi induces the knockdown of myc-RNF157 wild type, but fails to effectively induce knockdown of RNF157-Res. C) Cerebellar granule neurons were transfected at DIV 2 with the control vectors, RNF157 RNAi plasmid with control vector, or both RNF157 RNAi plasmid with myc-RNF157-Res expression plasmid together with a plasmid encoding ß-galactosidase. Neurons were subjected to immunocytochemistry using the ß-galactosidase antibody and the DNA dye bisbenzimide (Hoechst 33258) 
four days later. Percentage of apoptosis is presented as mean \pm SEM. While knockdown of RNF157 significantly increases apoptosis $\left(\mathrm{n}=4\right.$; ANOVA; $\left.{ }^{* * *} \mathrm{p}<0.001\right)$, expression of RNF157-Res significantly decreases apoptosis in the background of RNF157 RNAi ( $n=4$; ANOVA; ${ }^{* * *} \mathrm{p}<0.001$ ). A total of 2171 neurons were analyzed. wt, wild type; aa, amino acid.

\subsubsection{Overexpression of RNF157 protects granule neurons from cell death under apoptotic stimuli}

Having identified RNF157 as a pro-survival factor in neurons, I asked if overexpression of RNF157 protects neurons from cell death when exposed to apoptotic stimuli. In addition, to determine if the RING domain of RNF157 and thus E3 ligase activity is required for the pro-survival function of RNF157 in neurons, I generated an RNF157 mutant that lacks the RING domain (RNF157 $\Delta$ RING). Nicola Schwedhelm-Domeyer, MPIExperimental Medicine, Göttingen carried out gain-of-function experiments, in which she transfected cerebellar granule neurons at DIV 2 with the control vector, RNF157 wild type or RNF157 $\triangle$ RING expression plasmids together with a plasmid encoding ß-galactosidase. At DIV 5, conditioned media was replaced with BME 112 starvation media (= $\mathrm{KCl} /$ serum withdrawal) for $24 \mathrm{hr}$. Neurons were subsequently fixed and immunostained with the $ß$ galactosidase antibody. In control conditions, removal of conditioned media leads to an increase of the apoptotic rate to 72\%. Strikingly, expression of wild type RNF157 reduces the number of apoptotic neurons by $26.4 \%$, while RNF157 $\triangle$ RING fails to protect neurons from cell death (Figure 15). Together, these results suggest that RNF157 promotes neuronal survival in an E3 ligase activity-dependent manner.

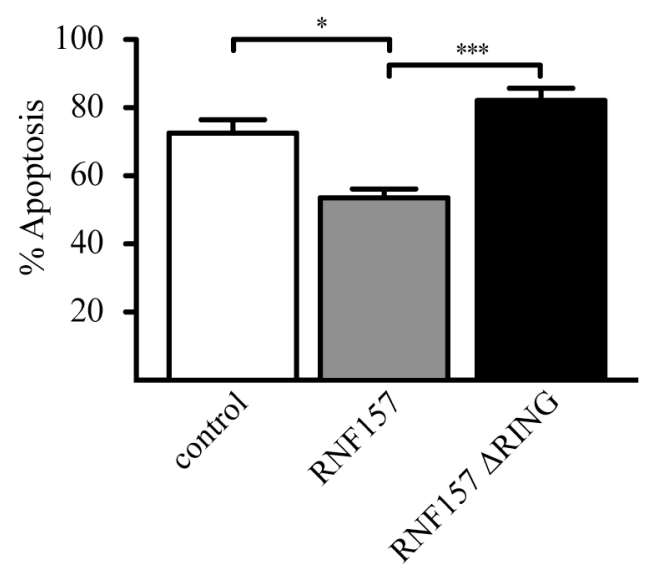

Figure 15. Overexpression of RNF157 promotes neuronal survival. Cerebellar granule neurons were transfected at DIV 2 with the control vector, myc-RNF157 wild type or myc-RNF157 $\Delta$ RING expression plasmids together with a plasmid encoding ß-galactosidase. Three days later, neurons were starved for $24 \mathrm{hr}$ and subjected to immunocytochemistry using the ß-galactosidase antibody and the DNA dye bisbenzimide (Hoechst 33258). Percentage of apoptosis is presented as mean \pm SEM. RNF157 overexpression significantly reduces apoptosis, while expression of RNF157 $\triangle$ RING fails to inhibit cell death in neurons (n=4; ANOVA; ${ }^{*} \mathrm{p}<0.05 ;{ }^{* * *} \mathrm{p}<0.001$ ). A total of 1383 neurons were analyzed. (Courtesy of N. Schwedhelm-Domeyer) 


\subsubsection{RNF157 promotes neuronal survival independently of the neuronal cell} type

Next, I determined whether the survival-promoting function of RNF157 is generalizable in mammalian neurons. Hence, I used cultured hippocampal neurons to analyze the effect of RNF157 loss-of-function in hippocampal neuron survival. Rat primary hippocampal neurons were isolated from E18 rat embryos and transfected at DIV 2 with the control vector or RNF157 RNAi plasmid together with a plasmid encoding ßgalactosidase. Neurons were subjected to survival assays at DIV 6. Here, I found that knockdown of RNF157 dramatically increases cell death to 98\% in hippocampal neurons as compared to control-transfected neurons (Figure 16). These results implicate that RNF157 regulates neuronal survival in a diverse population of neurons in the brain.

A

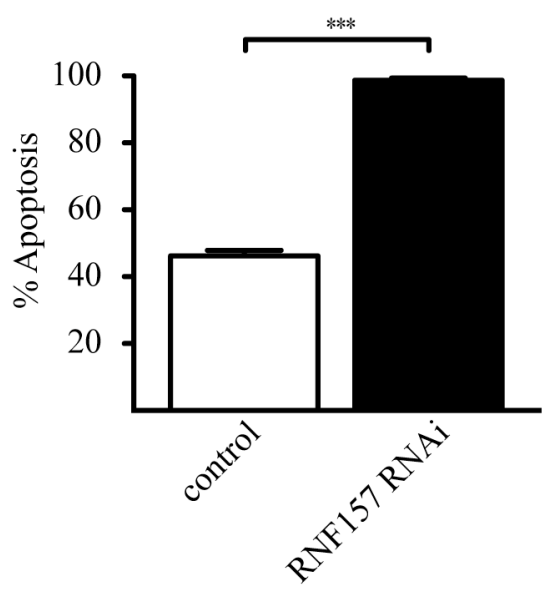

B

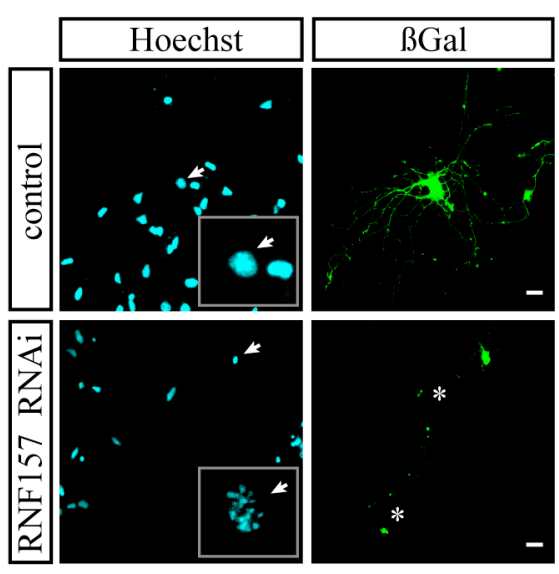

Figure 16. Knockdown of RNF157 dramatically increases apoptosis in hippocampal neurons. A) Hippocampal neurons transfected at DIV 2 with the control vector or RNF157 RNAi plasmid together with a plasmid encoding ß-galactosidase were subjected to immunocytochemistry using the ß-galactosidase (BGal) antibody and the DNA dye bisbenzimide (Hoechst 33258) four days later. Percentage of apoptosis is presented as mean \pm SEM. Apoptosis is significantly increased in RNF157 knockdown neurons as compared to control-transfected neurons ( $\mathrm{n}=3$; Student's t-test, ${ }^{* * *} \mathrm{p}<0.001$ ). A total of 655 neurons were analyzed. B) Representative images of neurons transfected with the control vector or RNF157 RNAi plasmid. RNF157 knockdown neurons show morphological characteristics of apoptosis (pyknotic nuclei, cellular breakdown leading to formation of apoptotic bodies). Arrows indicate nuclei of transfected neurons. Insets depict nuclei indicated with an arrow. Asterisks indicate apoptotic bodies. Scale bar equals $20 \mu \mathrm{m}$.

\subsubsection{RNF157 promotes dendrite development and maintenance in cerebellar} granule neurons

Recent literature has uncovered dual roles for several neurotrophic factors in neurons. One of those is the nerve growth factor (NGF) that is required for survival of sympathetic neurons, but also mediates axon outgrowth in vitro (Glebova \& Ginty, 2004). 
The latter was established by deleting the pro-apoptotic protein Bax that allowed the analysis of the survival-independent role of NGF in axon outgrowth. This prompted me to ask whether RNF157 could also be involved in neuronal morphogenesis in cerebellar granule neurons. To overcome RNF157 RNAi-induced apoptosis in neurons, I took advantage of the pro-survival Bcl-2 family member Bcl- $x_{\mathrm{L}}$ to perform morphological assays.

\subsubsection{Knockdown of RNF157 reduces dendritic but not axonal length in cerebellar} granule neurons

To study the role of RNF157 in neuronal morphogenesis, I performed loss-offunction analysis. I transfected cerebellar granule neurons at DIV 4 with the control vector, RNF157 RNAi plasmids II or III together with the Bcl- $\mathrm{x}_{\mathrm{L}}$ and GFP expression plasmids and subjected them to immunocytochemistry using the GFP antibody at DIV 8. Axons and dendrites of at least 30 GFP-positive neurons were analyzed per condition in at least 3 independent experiments using the ImageJ software. While RNF157 knockdown does not affect axonal length (Figure 17A), I found that neurons display a dramatic decrease in total dendritic length as compared to control-transfected neurons that developed a typical dendritic arbor (Figure 17 B/C).

To determine whether the RNF157 RNAi-induced dendritic phenotype is the result of the specific knockdown of RNF157 and not an off-target effect, I performed rescue experiments and used the previously validated RNF157-Res plasmid (Figure 14). Cerebellar granule neurons were transfected with the control vectors, RNF157 RNAi plasmid with control vector, or both RNF157 RNAi plasmid with RNF157-Res expression plasmid together with the Bcl- $\mathrm{x}_{\mathrm{L}}$ and GFP expression plasmids and subjected to immunocytochemistry using the GFP antibody at DIV 8. Morphological analysis revealed that expression of RNF157-Res in the background of RNF157 RNAi significantly restores dendritic length of RNF157 knockdown neurons to almost baseline level of controltransfected neurons (Figure 18). These experiments indicate that RNF157 regulates dendrite development in cerebellar granule neurons. 
A

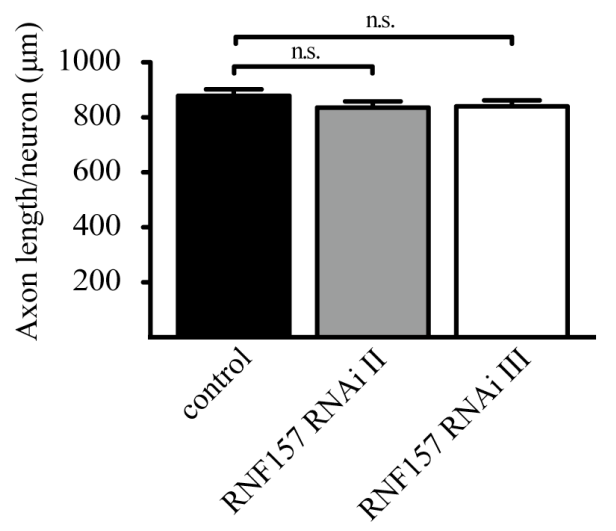

$\mathrm{B}$

$\mathrm{C}$
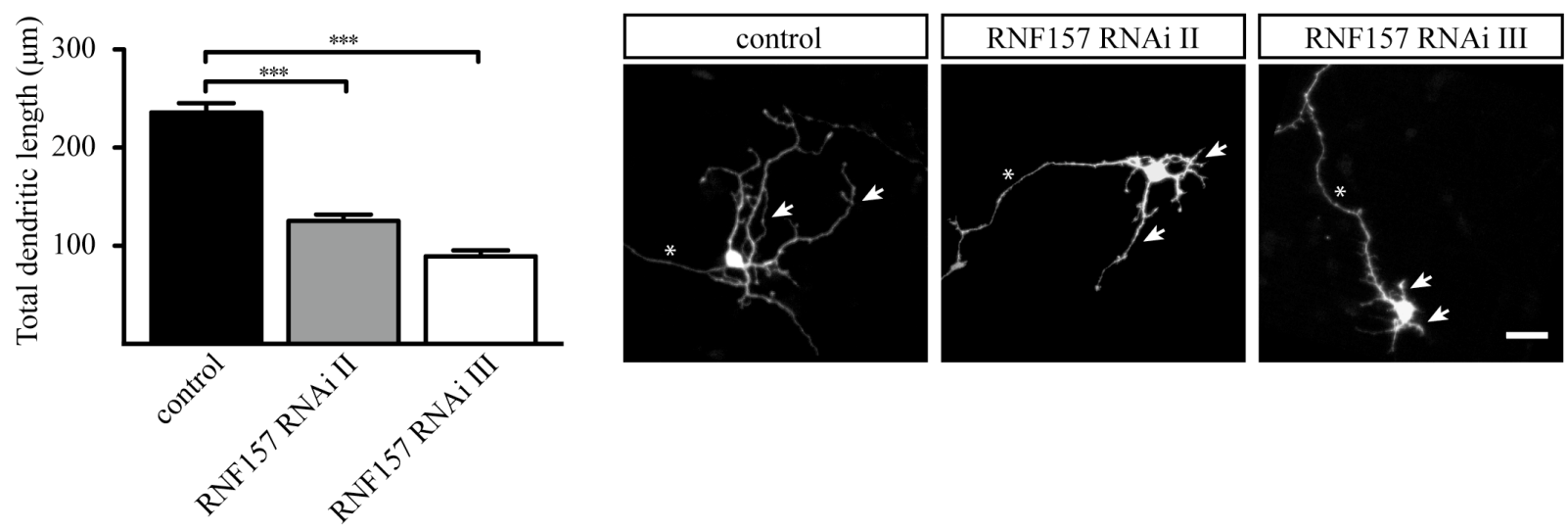

Figure 17. Knockdown of RNF157 impairs dendrite development but has no effect on axon growth. A) Cerebellar granule neurons transfected at DIV 4 with the control vector, RNF157 RNAi plasmids II or III together with the Bcl- $\mathrm{x}_{\mathrm{L}}$ and GFP expression plasmids were subjected to immunocytochemistry using the GFP antibody and the DNA dye bisbenzimide (Hoechst 33258) four days later. Axonal and total dendritic length were measured and presented as mean \pm SEM. Knockdown of RNF157 has little or no effect on axonal length in cerebellar granule neurons (n.s., not significant). B) Total dendritic length is significantly reduced in RNF157 knockdown neurons transfected with the RNF157 RNAi plasmids II or III as compared to controltransfected neurons $\left(\mathrm{n}=5\right.$; ANOVA; $\left.{ }^{* * *} \mathrm{p}<0.001\right)$. A total amount of 653 neurons were analyzed. C) Representative images of neurons transfected with the control vector, RNF157 RNAi plasmids II or III. Asterisks and arrows indicate axons and dendrites, respectively. Scale bar equals $20 \mu \mathrm{m}$.

A

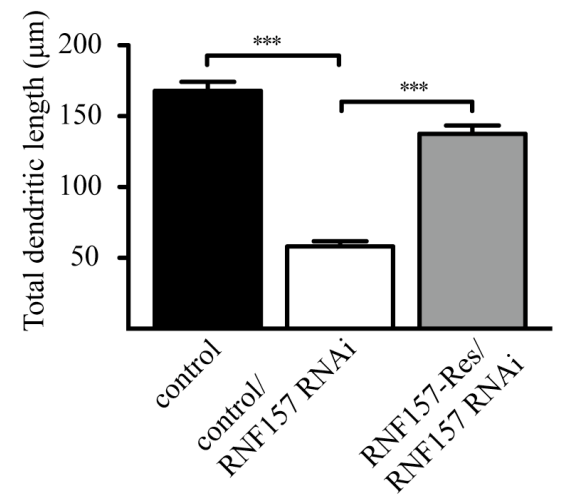

B
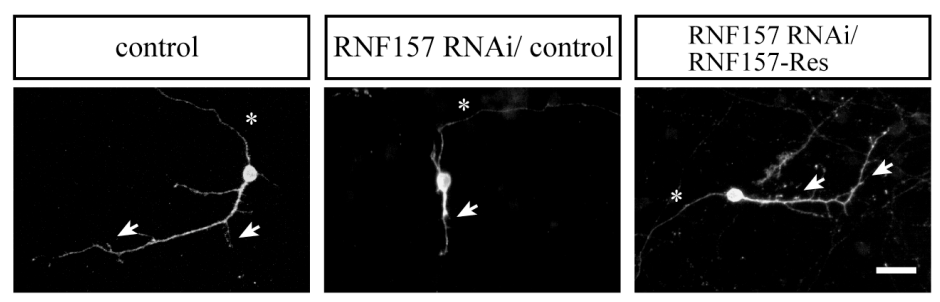

Figure 18. RNF157 is required for dendrite growth in neurons. A) Cerebellar granule neurons transfected at DIV 4 with the control vectors, RNF157 RNAi plasmid with control vector, or both RNF157 RNAi plasmid with myc-RNF157-Res expression plasmid together with the Bcl- $\mathrm{x}_{\mathrm{L}}$ and GFP expression plasmids were 
subjected to immunocytochemistry using the GFP antibody four days later. Expression of RNF157-Res in RNF157 knockdown neurons significantly restores total dendritic length as compared to control-transfected neurons ( $\mathrm{n}=3$; ANOVA; ${ }^{* * *} \mathrm{p}<0.001$ ). A total of 322 neurons were analyzed. B) Representative images of neurons transfected with the control vectors, RNF157 RNAi plasmid with control vector, or both RNF157 RNAi plasmid with RNF157-Res expression plasmid. Asterisks and arrows indicate axons and dendrites, respectively. Scale bar equals $20 \mu \mathrm{m}$.

\subsubsection{Knockdown of RNF157 reduces dendrite complexity in cerebellar granule} neurons

To further characterize the dendritic phenotype of the RNF157 RNAi, I performed Scholl analysis to determine the complexity of RNF157 knockdown neurons. Here, ImageJ software automatically counts the number of dendrites crossing concentric circles placed around the soma of a neuron at $7.5 \mu \mathrm{m}$ increments. At a radius of $22.5 \mu \mathrm{m}$ to $75 \mu \mathrm{m}$ from the cell body, RNF157 knockdown neurons show a significant reduction in the total amount of crossing dendrites as compared to control-transfected neurons (Figure 19). This analysis supports the role of RNF157 in dendrite growth and development.

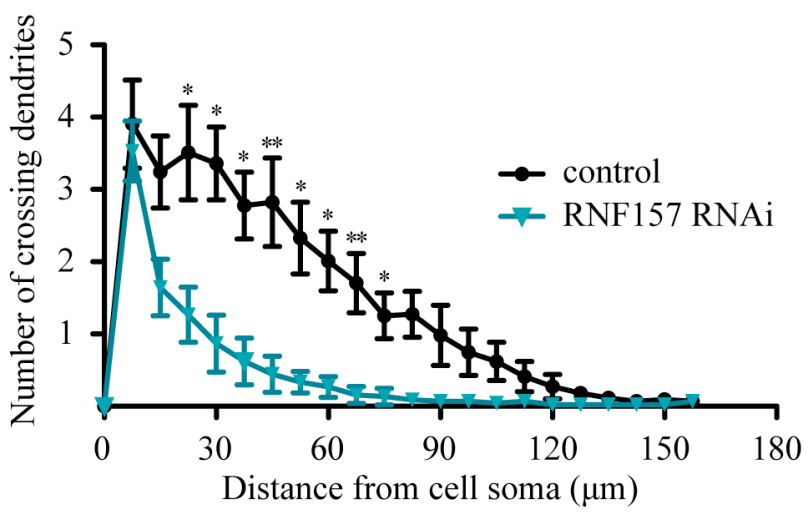

Figure 19. Knockdown of RNF157 reduces dendrite complexity. Cerebellar granule neurons transfected at DIV 4 with the control vector or RNF157 RNAi plasmid together with the Bcl- $\mathrm{x}_{\mathrm{L}}$ and GFP expression plasmids were subjected to immunocytochemistry using the GFP antibody four days later. Dendrite complexity was assessed by Scholl analysis and presented as mean \pm SEM. The numbers of crossing dendrites from 22,5 to $75 \mu \mathrm{m}$ around the cell soma are significantly reduced in RNF157 knockdown neurons as compared to control-transfected neurons ( $\mathrm{n}=3$, Student's t-test; $22,5 \mu \mathrm{m}{ }^{*} \mathrm{p}<0.05 ; 30 \mu \mathrm{m}{ }^{*} \mathrm{p}<0.05 ; 37,5 \mu \mathrm{m}$ $\left.{ }^{*} \mathrm{p}<0.05 ; 45 \mu \mathrm{m}{ }^{* *} \mathrm{p}<0.01 ; 52,5 \mu \mathrm{m}{ }^{*} \mathrm{p}<0.05 ; 60 \mu \mathrm{m}{ }^{*} \mathrm{p}<0.05 ; 67,5 \mu \mathrm{m}{ }^{* *} \mathrm{p}<0.01 ; 75 \mu \mathrm{m}{ }^{*} \mathrm{p}<0.05\right)$. A total of 89 neurons were analyzed.

\subsubsection{RNF157 is important for the maintenance of cerebellar granule neuron dendrites}

In further experiments, I addressed if RNF157 is crucial for the maintenance of dendrites after they have formed. To answer this, I transfected cerebellar granule neurons at DIV 4 with the control vector, RNF157 RNAi plasmids II or III together with the Bcl- $\mathrm{x}_{\mathrm{L}}$ and GFP expression plasmids and started measuring total dendritic length $24 \mathrm{hr}$ after 
transfection over a period of four consecutive days. At DIV 5, control neurons displayed a characteristic dendritic arborization that did not undergo further growth until DIV 8. Neurons transfected with the RNF157 RNAi plasmids II or III displayed dendritic morphology comparable to control-transfected neurons at DIV 5. However, while RNF157 RNAi plasmid II-transfected neurons still exhibit stable dendritic length at DIV 6, dendrites of RNF157 RNAi plasmid III-transfected neurons are significantly shorter. At DIV 7 and 8, dendrites of both RNF157 RNAi plasmids II and III-transfected neurons undergo striking shrinkage in comparison to control-transfected neurons (Figure 20). Given that the RNF157 RNAi III reduces RNF157 levels more efficiently as compared to the RNF157 RNAi II, the results also suggest a dose-dependent response of dendrites to loss-of-RNF157. In summary, my results indicate that RNF157 is important for the maintenance of dendrites in cerebellar granule neurons.

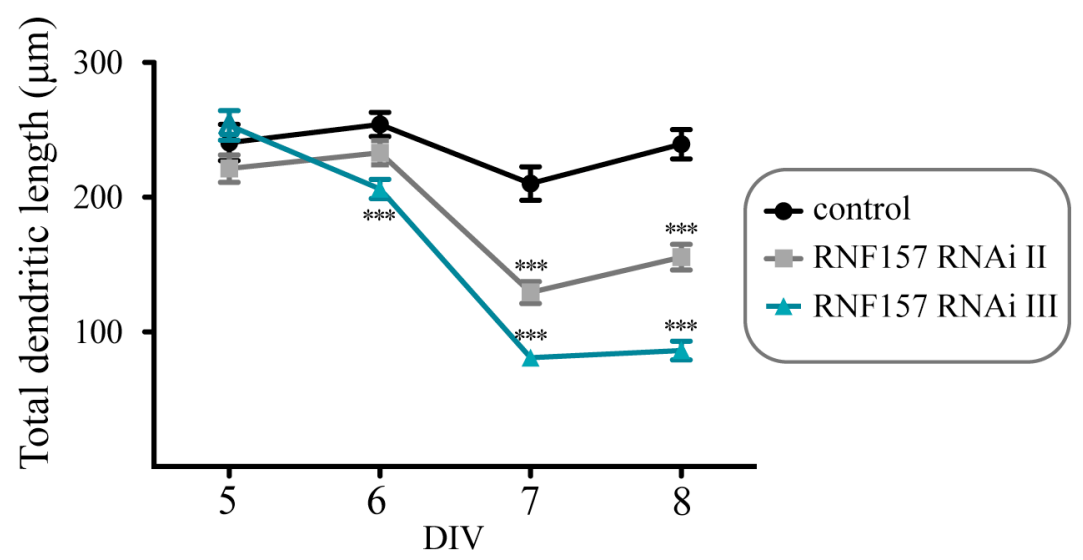

Figure 20. RNF157 is important for the maintenance of dendrites. Cerebellar granule neurons were transfected at DIV 4 with the control vector, RNF157 RNAi plasmids II or III together with the Bcl- $\mathrm{x}_{\mathrm{L}}$ and GFP expression plasmids. Neurons were fixed on the indicated days and subjected to immunocytochemistry using the GFP antibody. Total dendritic length was measured and presented as mean \pm SEM. The RNF157 RNAi II significantly decreases total dendritic length on DIV 7 and 8, while the RNF157 RNAi III significantly decreases dendritic length on DIV 6 and subsequent days as compared to control-transfected neurons (at least $\mathrm{n}=3$; ANOVA; ${ }^{* *} \mathrm{p}<0.001$ ). A total of 1550 neurons were analyzed.

\subsubsection{Introduction of the Wallerian degeneration slow fusion protein (Wlds) has no effect on the RNF157-knockdown induced dendritic phenotype}

Wlds was originally identified in the slow Wallerian degeneration mutant mouse, where it significantly delays injury-induced axonal degeneration as compared to wild type mice (Lunn et al, 1989). Wallerian degeneration slow fusion protein (Wlds) is a product of the rearrangement of two independent genes, which encode an in-frame fusion protein (Coleman et al, 1998) (Conforti et al, 2000). The N-terminus of Wlds is derived from the Nterminus of the E4-type ubiquitin ligase Ube4b, whereas the C-terminus displays the 
complete coding region of the nicotinamide mononucleotide adenyltransferase 1 (Nmnat 1), which are connected by an 18 aa linker (Coleman et al, 1998) (Mack et al, 2001). However, recent data suggests that expression of the Wlds in dorsal dendritic arborization neurons (ddaC) inhibits dendrite pruning as well as dendrite degeneration (Tao \& Rolls, 2011). In this study, I investigated whether overexpression of Wlds can block the reduction of cerebellar granule neuron dendrite length induced by knockdown of RNF157.

Thus, I transfected cerebellar granule neurons at DIV 4 with the control vectors, Wlds expression plasmid with control vector, RNF157 RNAi plasmid with control vector, or both RNF157 RNAi plasmid with Wld ${ }^{s}$ expression plasmid together with the Bcl- $\mathrm{x}_{\mathrm{L}}$ and GFP expression plasmids. At DIV 8, morphological analysis revealed that overexpression of Wlds has little or no effect on cerebellar granule neuron dendrite length as compared to controltransfected neurons. In addition, expression of Wlds in RNF157 knockdown neurons does not restore the RNF157 RNAi-induced reduction in total dendritic length (Figure 21). These results suggest that Wlds does not operate downstream of RNF157 in the regulation of granule neuron dendrite growth.

A

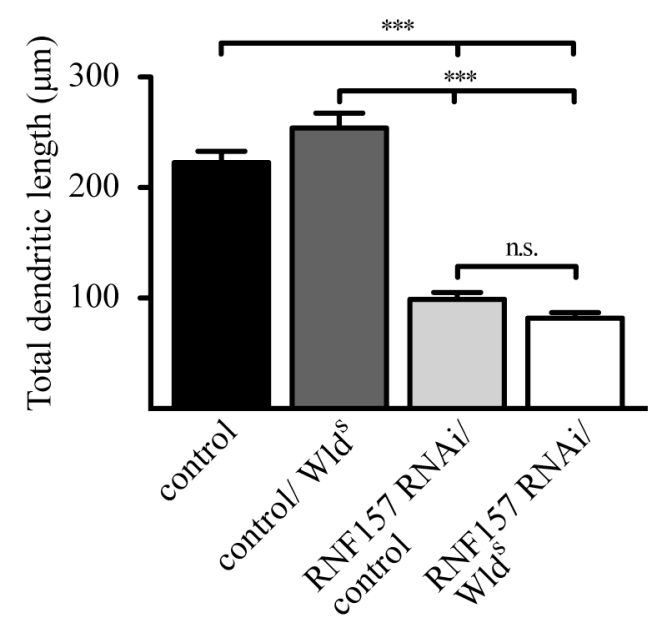

B
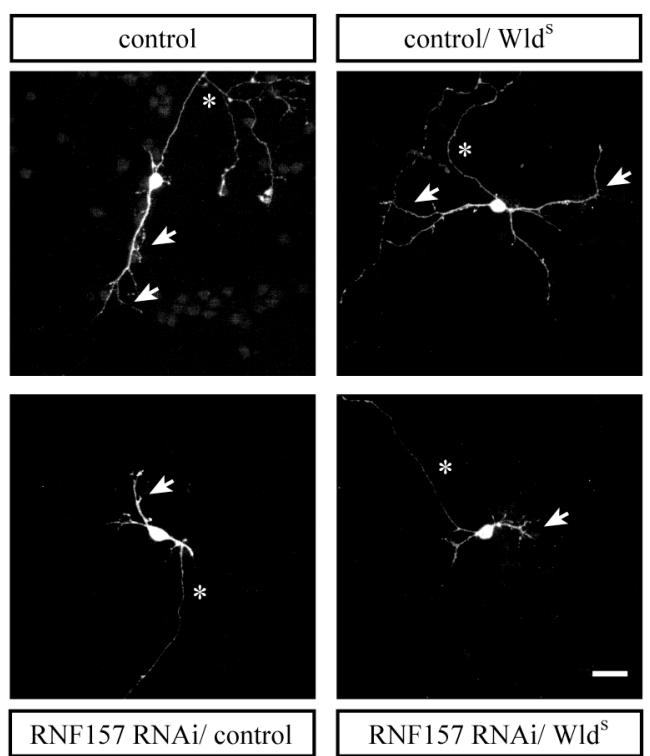

Figure 21. Wlds has no effect on the RNF157-knockdown induced dendritic phenotype. A) Cerebellar granule neurons transfected at DIV 4 with the control vectors, myc-Wlds expression plasmid with control vector, RNF157 RNAi plasmid with control vector, or both RNF157 RNAi plasmid with myc-Wlds expression plasmid together with the Bcl- $\mathrm{x}_{\mathrm{L}}$ and GFP expression plasmids were subjected to immunocytochemistry using the GFP antibody four days later. Total dendritic length was measured and presented as mean \pm SEM. Expression of Wlds in RNF157 knockdown neurons has little or no effect on total dendritic length as compared to RNF157 RNAi-transfected neurons (n=3, ANOVA; n.s., not significant; *** ${ }^{*}<0.001$ ) A total of 571 neurons were analyzed. B) Representative images of neurons transfected with the control vectors, Wlds expression plasmid with control vector, RNF157 RNAi plasmid with control vector, or both RNF157 RNAi 
plasmid with Wlds expression plasmid. Asterisks and arrows indicate axons and dendrites, respectively. Scale bar equals $20 \mu \mathrm{m}$.

\subsubsection{RNF157 significantly increases dendritic but not axonal length in an E3 ligase activity-independent manner in cerebellar granule neurons}

Having established that RNF157 promotes dendrite morphogenesis and complexity, I asked whether overexpression of RNF157 would trigger a gain-of-function effect in dendrite development. In addition, I addressed whether a potential gain-of-function effect is dependent on RNF157's E3 ligase activity. To answer this question, I transfected cerebellar granule neurons at DIV 4 with the control vector, RNF157 wild type or RNF157 $\triangle$ RING expression plasmids together with the Bcl- $\mathrm{x}_{\mathrm{L}}$ and GFP expression plasmids and analyzed dendrite morphology at DIV 8.

A

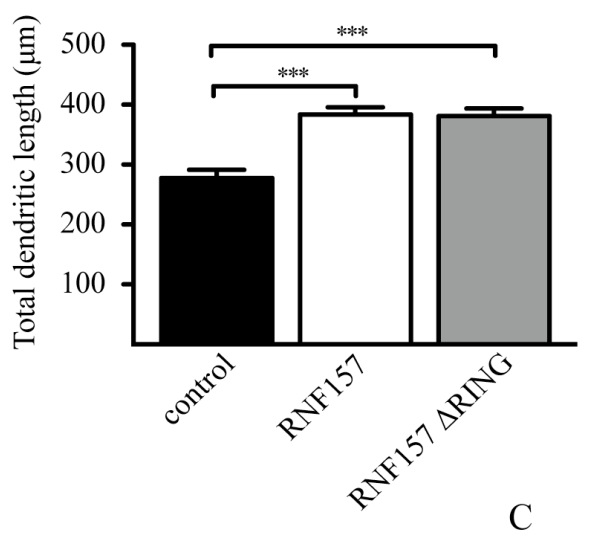

B
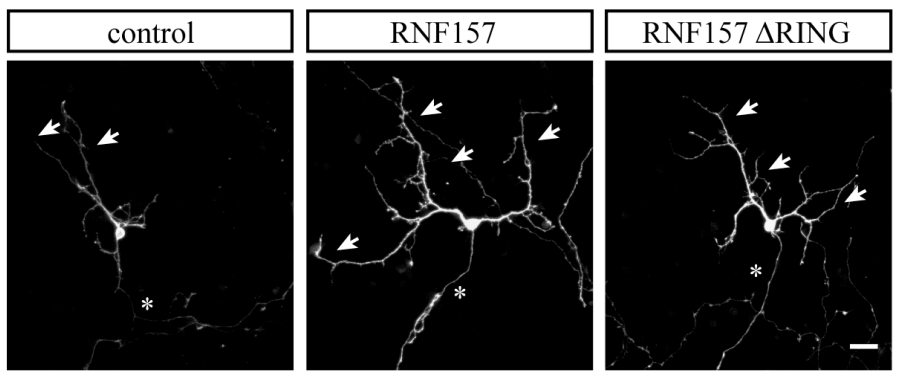

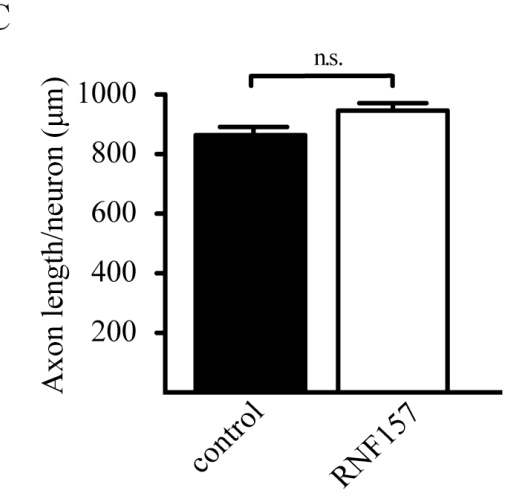

Figure 22. Overexpression of RNF157 increases dendritic but not axonal length in an E3 ligase activity-independent manner. Cerebellar granule neurons transfected at DIV 4 with the control vector, myc-RNF157 wild type or myc-RNF157 $\triangle$ RING expression plasmids together with the Bcl- $x_{\mathrm{L}}$ and GFP expression plasmids were subjected to immunocytochemistry using the GFP antibody four days later. Axonal and total dendritic length were measured and presented as mean \pm SEM. A) Overexpression of RNF157 and RNF157 $\triangle$ RING significantly increases total dendritic length as compared to control-transfected neurons $\left(\mathrm{n}=3\right.$; ANOVA; ${ }^{* * *} \mathrm{p}<0.001$; n.s., not significant). A total of 315 neurons were analyzed. B) Representative images of control, myc-RNF157 and myc-RNF157 $\triangle$ RING transfected neurons. Asterisks and arrows indicate axons and dendrites, respectively. Scale bar equals $20 \mu \mathrm{m}$. C) Overexpression of RNF157 has little or no effect on axonal length (n.s.; not significant). A total of 267 neurons were analyzed. 
Morphological analysis revealed that overexpression of RNF157 and RNF157 $\Delta$ RING significantly increases dendritic length as compared to control-transfected neurons (Figure 22A/B). Furthermore, overexpression of RNF157 has little or no effect on axonal length (Figure 22C). These results indicate that RNF157 selectively promotes dendritic but not axonal morphogenesis in an E3 ligase activity-independent manner.

\subsubsection{Overexpression of RNF157 influences dendritic arborization}

In addition to the RNF157 overexpression-induced increase in total dendritic length, I noticed a more elaborate dendritic tree. To confirm this observation, I performed Scholl analysis. At a radius of $45 \mu \mathrm{m}$ to $85.5 \mu \mathrm{m}$ from the cell body, RNF157 overexpressing neurons show a significant increase in the total amount of crossing dendrites as compared to control-transfected neurons (Figure 23). These results suggest that in addition to dendrite growth regulation and maintenance, RNF157 affects the complexity of cerebellar granule neuron dendrites.

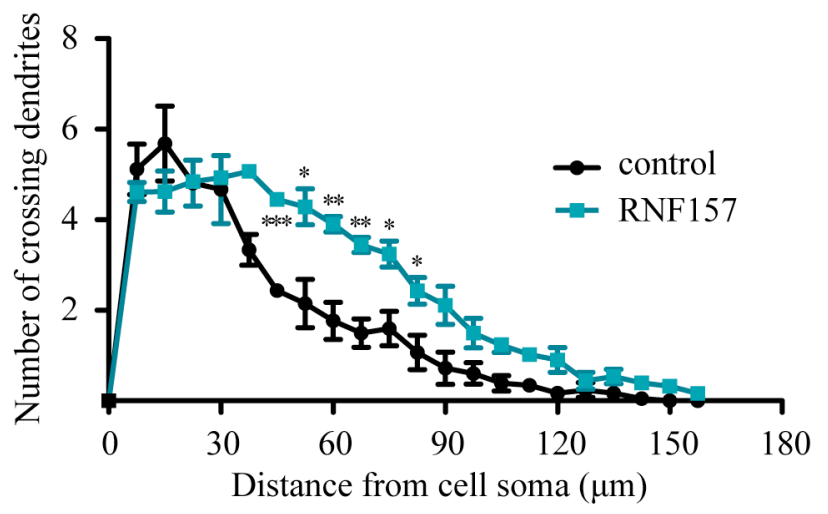

Figure 23. Overexpression of RNF157 increases dendrite complexity. Cerebellar granule neurons transfected at DIV 4 with the control vector or RNF157 RNAi plasmid together with the Bcl- $\mathrm{x}_{\mathrm{L}}$ and GFP expression plasmids were subjected to immunocytochemistry using the GFP antibody four days later. Dendrite complexity was assessed by Scholl analysis and presented as mean \pm SEM. The numbers of crossing dendrites from 45 to $85,5 \mu \mathrm{m}$ around the cell soma are significantly increased in RNF157-overexpressing neurons as compared to control-transfected neurons ( $n=3$, Student's t-test; $45 \mu \mathrm{m}{ }^{* * *} \mathrm{p}<0.001 ; 52,5 \mu \mathrm{m}$ $\left.{ }^{*} \mathrm{p}<0.05 ; 60 \mu \mathrm{m}{ }^{* *} \mathrm{p}<0.01 ; 67,5 \mu \mathrm{m}{ }^{* *} \mathrm{p}<0.01 ; 75 \mu{ }^{*} \mathrm{p}<0.05 ; 82,5 \mu \mathrm{m}{ }^{*} \mathrm{p}<0.05\right)$. A total of 84 neurons were analyzed.

\subsubsection{RNF157 promotes dendrite development independently of the neuronal cell} type

To establish whether RNF157-regulated dendrite morphogenesis is a generalizable mechanism, I determined whether the RNF157-knockdown induced dendritic phenotype is true for other neuronal cell types. Here, I used cultured hippocampal neurons to analyze the influence of RNF157 knockdown on dendritic as well as on axonal morphology. Rat 
primary hippocampal neurons were isolated from E18 embryos and transfected at DIV 4 with a bicistronic control vector or RNF157 RNAi plasmid additionally expressing GFP under the control of a CMV promoter. Hippocampal neurons were subjected to morphological analysis at DIV 8. While the RNF157 RNAi has little or no effect on axonal length (Figure 24A), these neurons exhibit a striking reduction in dendritic length. RNF157 knockdown neuron dendrites are 85\% shorter than control-transfected neurons (Figure 24B/C). These results identify RNF157 as a regulator of dendrite morphogenesis independently of the neuronal cell type.

A

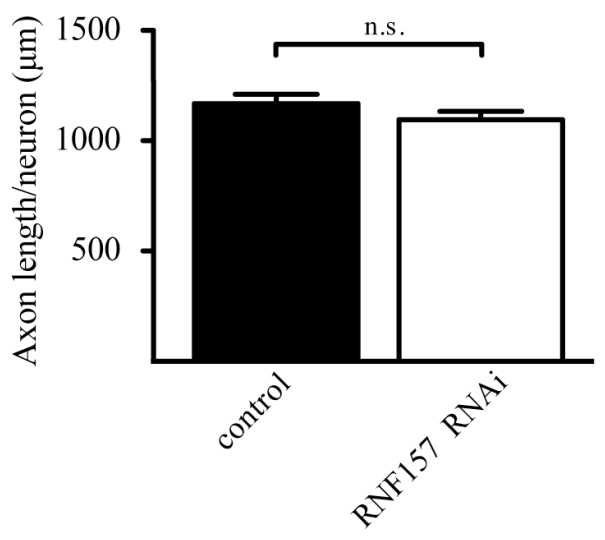

B

$\mathrm{C}$
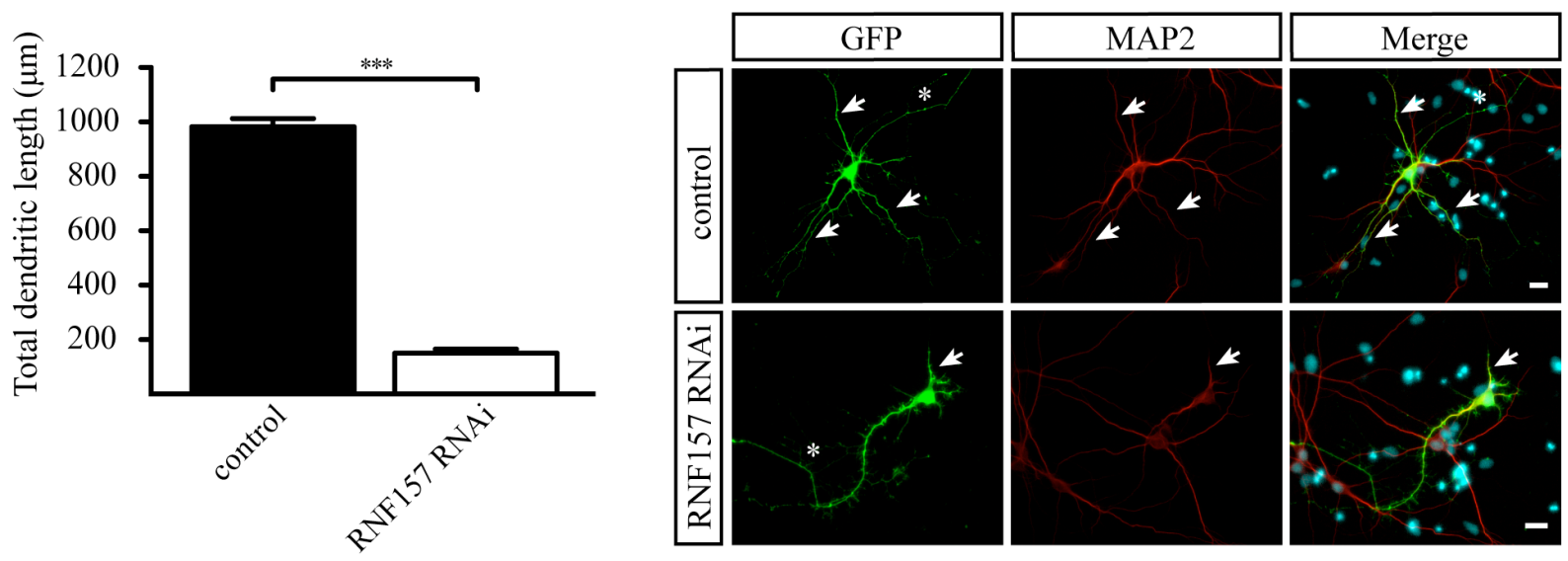

Figure 24. Knockdown of RNF157 dramatically decreases dendritic but not axonal length in hippocampal neurons. Hippocampal neurons transfected at DIV 4 with the bicistronic control vector or RNF157 RNAi plasmid together with a plasmid encoding Bcl- $\mathrm{x}_{\mathrm{L}}$ were subjected to immunocytochemistry using the GFP antibody four days later. Axonal and total dendritic length were measured and presented as mean \pm SEM. A) Knockdown of RNF157 has little or no effect on axonal length in hippocampal neurons (n.s., not significant). B) Total dendritic length is significantly reduced in RNF157 knockdown neurons as compared to control-transfected neurons $\left(n=3\right.$; Student's t-test; $\left.{ }^{* * *} \mathrm{p}<0.001\right)$. A total amount of 189 neurons were analyzed. C) Representative images of hippocampal neurons transfected with the bicistronic control vector or RNF157 RNAi plasmid. Asterisks and arrows indicate axons and dendrites, respectively. Scale bar equals $20 \mu \mathrm{m}$. 


\subsection{Identification of RNF157 interacting proteins in the mammalian} brain

\subsubsection{Yeast Two-Hybrid screen}

E3 ligases confer substrate specificity in the process of ubiquitination. Here, RING E3 ligases act as scaffold proteins to mediate the transfer of ubiquitin from the E2 ubiquitin-conjugating enzyme onto the substrate. To identify proteins that directly interact with RNF157, I performed a yeast two-hybrid screen using a whole brain mouse cDNA library pooled from $200 \mathrm{BALB} / \mathrm{c}$ males at the age of 9-12 weeks. Hence, three different RNF157 bait plasmids were cloned, covering the whole sequence of RNF157 as follows:

1) pGBT9 - -RNF1571-307 (N-terminus of RNF157 including RING domain)

2) pGBT9 - RNF157 $298-644$, (C-terminus of RNF157)

3) pGBT9 $9_{L}-R_{N F 157}$ 231-311, (RING domain of RNF157)

After smale-scale transformation of the yeast strain GC1945 with the different bait plasmids, I analyzed if the bait plasmids autonomously activate the HIS3 reporter gene by plating the transformed yeast cells on agar plates without histidine. Since the $\mathrm{pGBT} \mathrm{L}_{\mathrm{L}}$ RNF1571-307 and pGBT9L-RNF157298-644 showed autoactivation of the HIS3 reporter gene, transformed yeast cells were additionally plated on minus-HIS plates with different concentrations (5-25 mM) of 3-amino-1, 2, 4-triazole (3-AT), which is a competitive inhibitor of the product of the HIS3 gene. Only the pGBT9L-RNF157298-644 bait plasmid still showed strong autoactivation of the HIS3 reporter gene when exposed to high levels of 3AT. Subsequently, I used the pGBT9L-RNF157 1 1-307 and the pGBT9 - RNF157 $231-311$ to perform the screen. After large-scale transformation with the prey cDNA library, transformed yeast cells were plated on agar plates without tryptophan, leucine and histidine and growing colonies were additionally analyzed by a ß-galactosidase (ßGal)assay to eliminate false-positive interactions. Positive clones were analyzed by PCR using pACT2 specific primers and subsequently sequenced. In both conditions positive clones were identified. Interactions of identified candidates with full length RNF157 were confirmed using co-immunoprecipitation analysis. 


\subsection{Fe65, a novel interactor of RNF157, acts in concert with RNF157 in the control of neuronal survival}

Among the novel identified RNF157 interactors, I decided to focus on the adapter protein Fe65. Fe65, which is a member of a small family the multimodular scaffolding proteins including Fe65L1 and Fe65L2, is enriched in the brain (Bressler et al, 1996; Duilio et al, 1991). In neurons, Fe65 is present in the cytoplasm and the nucleus (Hoe et al, 2006). Together with Mena (a regulator of actin dynamics) and amyloid precursor protein (APP), Fe65 forms a trimeric complex and influences cell motility, axon branching and growth cone formation (Ermekova et al, 1997; Ikin et al, 2007; Sabo et al, 2001; Sabo et al, 2003). Furthermore, Fe65 plays an important role in DNA repair with APP and the histone acetyltransferase Tip60 (Stante et al, 2009). On this basis, Fe65 emerged as an interesting candidate and I addressed whether Fe65 could be a possible substrate of RNF157 and if they act in concert to control dendrite development or neuronal survival.

\subsubsection{Fe65 is a novel interactor of RNF157}

To validate the interaction of RNF157 and Fe65, I carried out coimmunoprecipitation analysis. I transfected HEK 293T cells with expression plasmids for myc-RNF157, Flag-Fe65 and the respective control vectors. Lysates were subjected to immunoprecipitation with the Fe65 antibody and subsequently analyzed by immunoblotting with the myc antibody to visualize RNF157. In a reciprocal experiment, I immunoprecipitated RNF157 followed by immunoblotting with the Fe65 antibody. These experiments confirmed the interaction of RNF157 with the adaptor protein Fe65 (Figure 25A/B). Supplementary, co-immunoprecipitation analysis revealed no interaction of RNF157 with Fe65L1 (data not shown). In addition, Anna Holoubowska validated the endogenous interaction of RNF157 and Fe65 in mouse P6 cerebellum and P12 cortex (Figure 25C/D). Thus, Fe65 is a novel interactor of RNF157. 
A

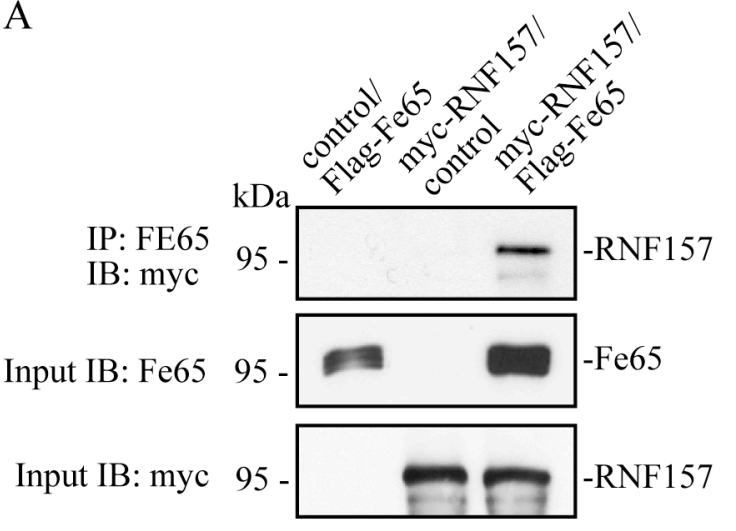

C

IB: RNF157

Cerebellum P6

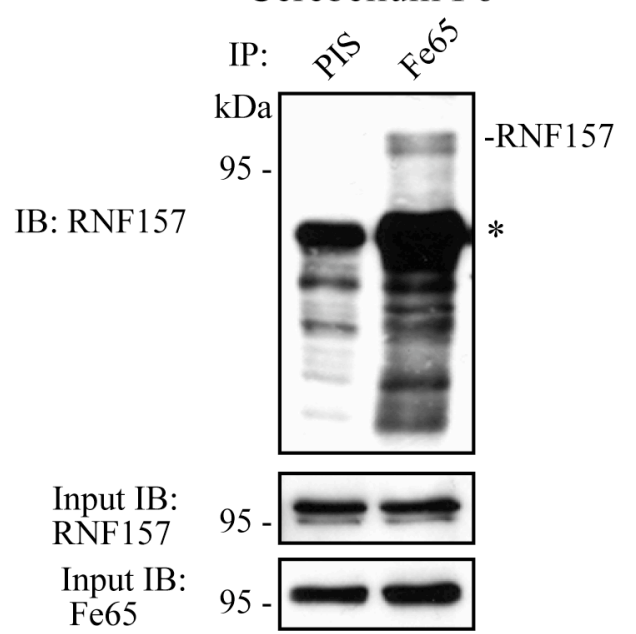

B

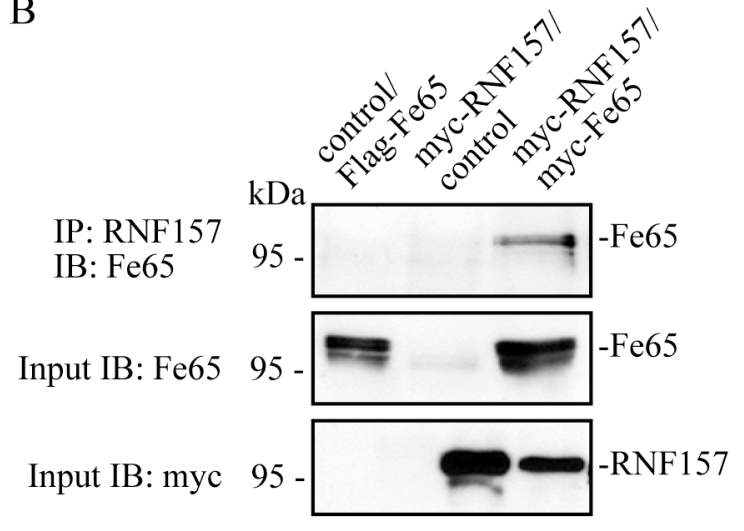

D
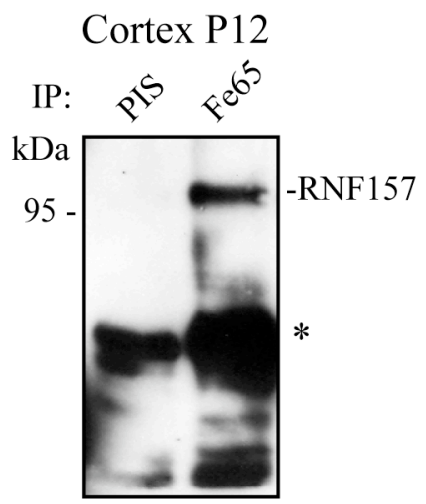

Input IB: RNF157 Input IB: Fe65

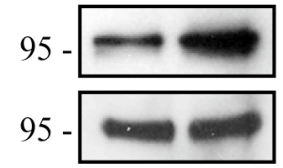

Figure 25. Fe65 interacts with RNF157 in heterologous cells and in vivo. A/B) Lysates of HEK 293T cells transfected with Flag-Fe65 or myc-RNF157 together with control vector, or both Flag-Fe65 and myc-RNF157 were immunoprecipitated using the Fe65 or RNF157 antibodies respectively and immunoblotted with the indicated antibodies. C/D) Crude lysates of P6 rat cerebellum or P12 mouse cortex were immunoprecipitated with a preimmune serum (PIS) or the Fe65 antibody followed by immunoblotting with the RNF157 antibody (Courtesy of A. Holubowska). Asterisk, IgG I $_{\text {; }}$ B, immunoblot; IP, immunoprecipitation.

\subsubsection{Mapping the specific binding regions of RNF157 and Fe65}

To map the regions of Fe65 and RNF157 that are required for their interaction, Dr. Judith Stegmüller, MPI of Experimental Medicine, Göttingen, cloned truncation mutants of RNF157 and Fe65. Co-immunoprecipitation experiments to identify the RNF157 binding region in Fe65 were performed by Shih-Ju Lee, those to identify the Fe65 binding region in RNF157 by Nicola Schwedhelm-Domeyer. RNF157 interacts with Fe65 fragments that harbor the second PTB domain (Fe65aa525-711 as well as to Fe65aa325-711). In addition, the binding of RNF157 with Fe65aa325-711 seemed to be stronger than with Fe65aa525-711 (Figure 26A). Conversely, Fe65 interacts with RNF157 aa1-237 and RNF157 aa238-442 suggesting that the Fe65 binding region was divided into two parts (Figure 26B). Furthermore, Fe65 interacts 
with RNF157 in an RING-domain independent manner (Figure 26B). Hence, these results indicate that the Fe65 binding region is located N-terminally in front of the RING domain, while the C-terminal fragment with $2^{\text {nd }}$ PTB domain of Fe65 binds to RNF157 (Figure 26C).

A

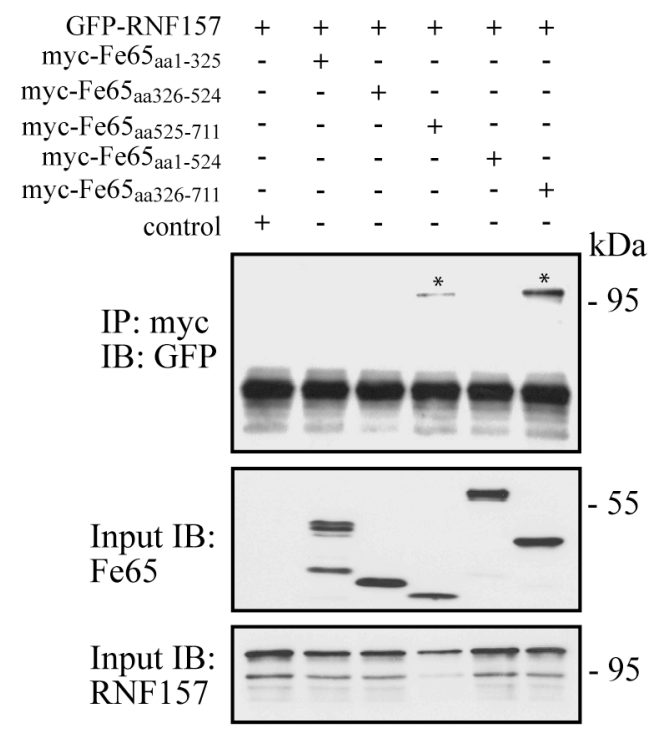

B

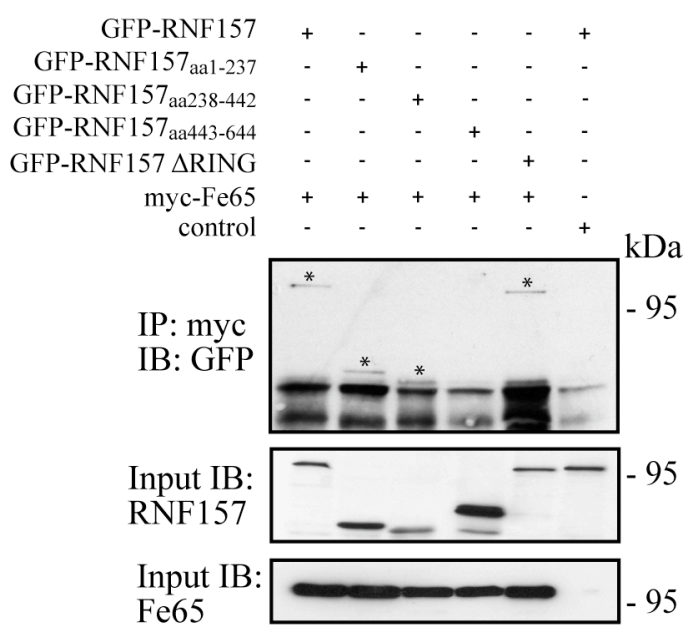

$\mathrm{C}$

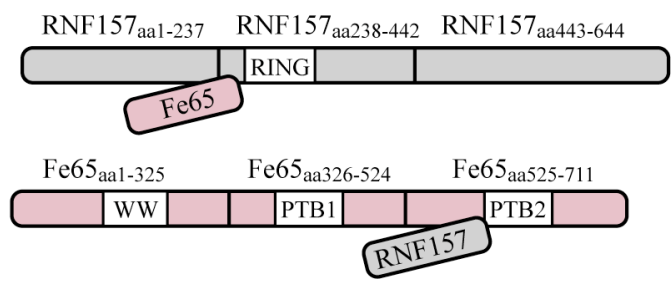

Figure 26. Mapping the interacting regions of RNF157 and Fe65. A/B) Lysates of HEK 293T cells transfected with the indicated plasmids were immunoprecipitated using the myc antibody followed by immunoblotting with the GFP antibody. (A= courtesy of S.J. Lee; B= courtesy of N. Schwedhelm-Domeyer). C) Schematic representation of the interaction domains of RNF157 and Fe65. IB, immunoblot; IP, immunoprecipitation. Asterisks indicate positive interactions.

\subsubsection{Expression and localization of Fe65 during brain development}

The expression of Fe65 was examined in cultured primary neurons and in the developing brain. I examined the expression of Fe65 in rat primary cerebellar neurons isolated from P6 rat pups and in primary cortical neurons prepared from E18 rat embryos. Western blot analysis revealed a strong expression of Fe65 from DIV 2-6 in cerebellar granule neurons, whereas cortical neurons display a weaker expression (Figure 27A). Next, I analyzed the expression of Fe65 in tissue lysates of mouse cerebellum, cortex and hippocampus isolated at different ages. In the cerebellum, I observed Fe65 expression as early as E18 with an increase of expression at later postnatal ages (P9-14) and the highest 
expression level in adulthood. While Fe65 expression in cortex is weaker and mostly unchanged throughout development, Fe65 expression in hippocampus is highest at E18-P0 with a subsequent decrease of expression at older stages (Figure 27B).

A
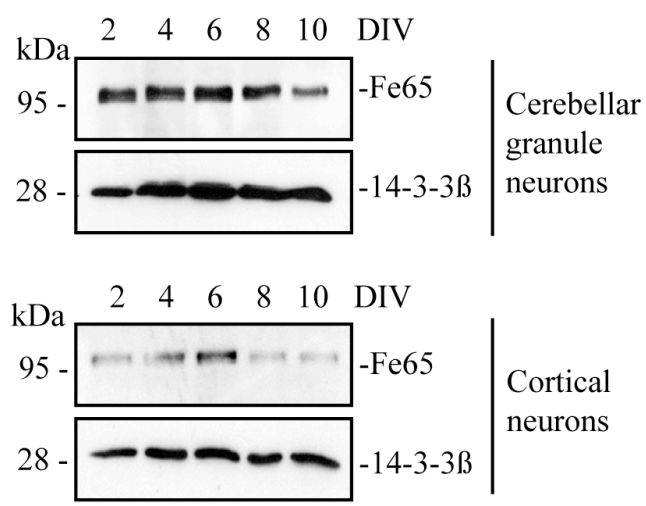

B
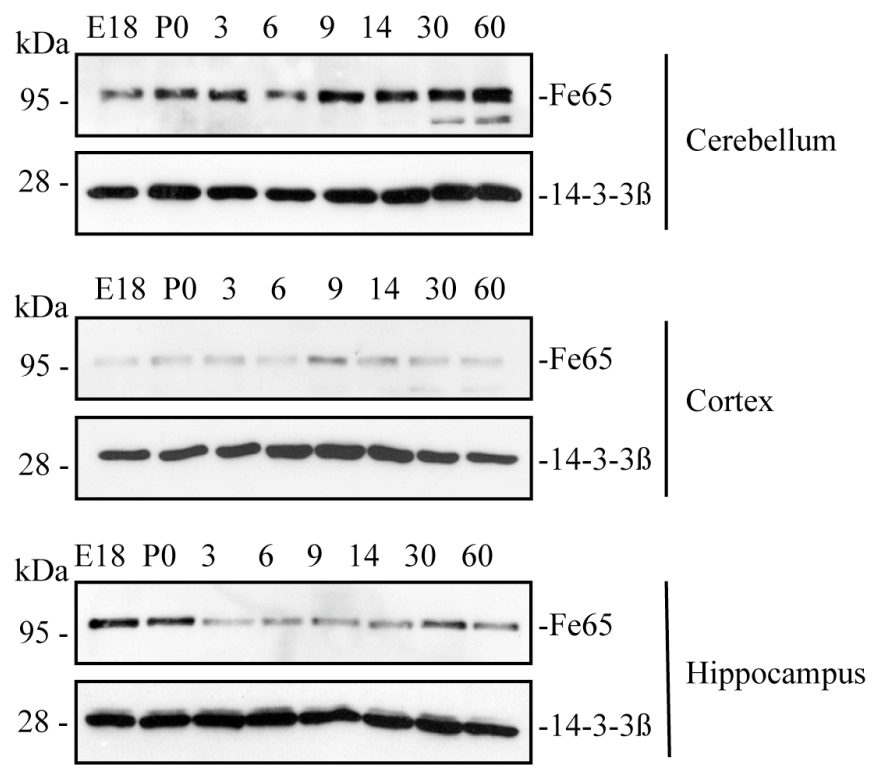

Figure 27. Developmental expression of Fe65 in the brain. A) Lysates of cerebellar granule and cortical neurons prepared from P6 or E18 rats respectively and placed in culture for the indicated days were immunoblotted using the Fe65 and 14-3-3ß antibodies. The latter served as loading control. B) Crude lysates of mouse cerebellum, cortex and hippocampus isolated at the indicated days (E, embryonic; $\mathrm{P}$, postnatal day) were subjected to immunoblotting using the Fe65 antibody. 14-3-3ß served as loading control.

To examine the subcellular localization of Fe65, I subjected DIV 6 cerebellar granule neurons to immunocytochemistry using the Fe65 antibody. Intracellular localization of Fe65 was detected in the cytoplasm of cell bodies, the processes and in the nucleus of cerebellar granule neurons (Figure 28). In summary, I found that Fe65 is expressed throughout development and localizes to the cytoplasm, processes and the nucleus in neurons.
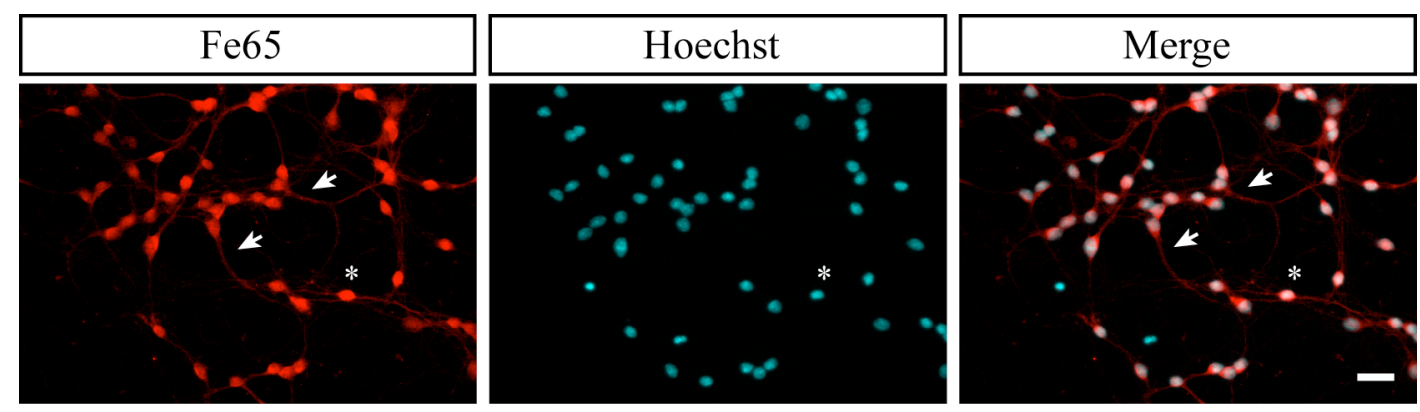

Figure 28. Subcellular localization of Fe65 is confined to the somatoneuritic and nuclear compartment. Cerebellar granule neurons at DIV 6 were subjected to immunocytochemistry using the Fe65 
antibody and the DNA dye bisbenzimide (Hoechst 33258). Arrows and asterisks indicate processes and nuclei, respectively. Scale bar equals $20 \mu \mathrm{m}$.

\subsubsection{Ubiquitination of Fe65 in cerebellar granule neurons}

Given that Fe65 interacts with RNF157, I asked whether Fe65 is ubiquitinated and degraded via the ubiquitin-proteasome system in neurons. Hence, cerebellar granule neurons were treated at DIV 2 with vehicle or lactacystin (selective inhibitor of the proteasome) and Fe65 expression was assessed in Western blot experiments. Here, Fe65 protein levels were increased upon proteasome inhibition (Figure 29A). Next, we examined if Fe65 is ubiquitinated in the rodent brain and how the ubiquitin moieties are linked to Fe65. Dr. Judith Stegmüller, MPI of experimental Medicine, Göttingen subjected cerebellar granule neurons at DIV 7 to immunoprecipitation using either the control (HA antibody) or Fe65 antibodies. Immunoblotting with an ubiquitin specific antibody revealed that Fe65 is indeed polyubiquitinated in neurons (Figure 29B).
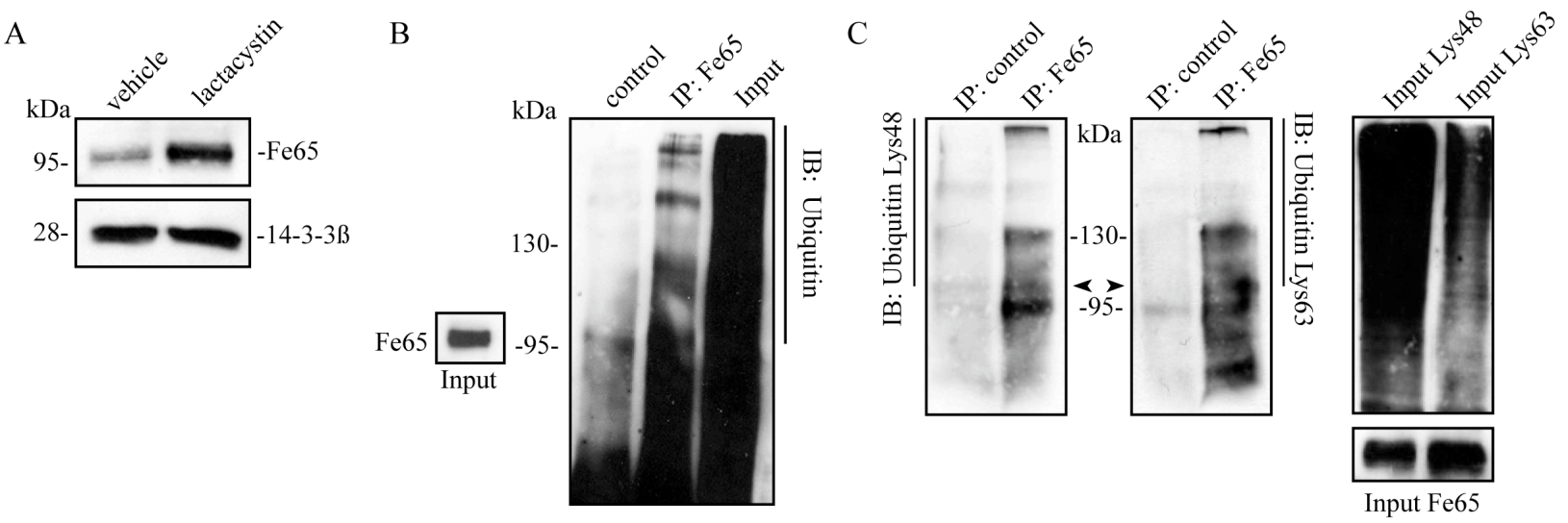

Figure 29. Ubiquitination of Fe65 in neurons. A) Lysates of cerebellar granule neurons at DIV 2 treated with $20 \mu \mathrm{m}$ lactacystin or vehicle for $5 \mathrm{hr}$ were subjected to immunoblotting using the Fe65 and 14-3-3ß antibodies. The latter served as loading control. B) Cerebellar granule neurons at DIV 7 were subjected to immunoprecipitation using the HA (control) or Fe65 antibodies followed by immunoblotting with an ubiquitin antibody (Courtesy of J. Stegmüller). C) Cerebellar granule neurons at DIV 6 were subjected to immunoprecipitation using the GFP (control) or Fe65 antibodies followed by immunoblotting using the Lys48-or Lys63-specific ubiquitin antibodies. IB, immunoblot; IP, immunoprecipitation. Arrowheads indicate Fe65.

In further experiments, I examined the nature of the polyubiquitin chains linked to Fe65. Ubiquitin harbors seven different lysine residues (Lys 6, 11, 27, 29, 33, 48 and 63) by which it can be conjugated to the target substrate or to another ubiquitin molecule (Goto et al, 2010) (Peng et al, 2003). While polyubiquitin chains linked via Lys48 target proteins for proteasomal degradation, Lys63-linked ubiquitin chains mediate functional protein modifications (Bennett et al, 2007). To analyze if Fe65 is targeted for proteasomal 
degradation or ubiquitinated in a non-degradative manner, I used antibodies that specifically recognize either Lys48-or Lys63-linked ubiquitin chains. Thus, cerebellar granule neurons were subjected at DIV 6 to immunoprecipitation using the control GFP or Fe65 antibodies. Both, immunoblotting with Lys48-and Lys63-ubiquitin specific antibodies produced a polyubiquitination smear (Figure 29C). These results suggest that Fe65 is modified by the attachment of Lys48-and Lys63-linked polyubiquitin chains. In summary, we could show that Fe65 is ubiquitinated and degraded in a proteasome-dependent manner and undergoes non-degradative ubiquitin modification that may alter its localization, activity or interaction with binding partners (Schnell \& Hicke, 2003).

\subsubsection{Fe65 is ubiquitinated by RNF157}

Next, we set out to investigate if RNF157 exhibits autoubiquitination activity and subsequently if Fe65 may serve as a novel target of RNF157. These experiments were carried out by Nicola Schwedhelm-Domeyer. To analyze whether RNF157 would catalyze ubiquitination, in vitro ubiquitination assays were performed. Myc-RNF157 was immunopurified from Neuro 2A cells and incubated in the presence of ATP, E1, different E2s and ubiquitin. Immunoblotting of the reaction products with an ubiquitin antibody revealed that RNF157 in a complex with the E2 UbcH5a exhibits a polyubiquitination smear (Figure 30A). This result suggests that RNF157 harbors autoubiquitination activity in vitro.

Next, both RNF157 and Fe65 were subjected to in vitro ubiquitination assays. Immunopurified Flag-Fe65 was incubated in the presence of ATP, E1, different E2s, ubiquitin and immunopurified RNF157. Here, immunoblotting of the reaction products with the Flag antibody revealed that RNF157 in a complex with the E2 UbcH5a mono-or diubiquitinates Fe65 in vitro (Figure 30B). This result suggests that Fe65 is a target of RNF157. 
A

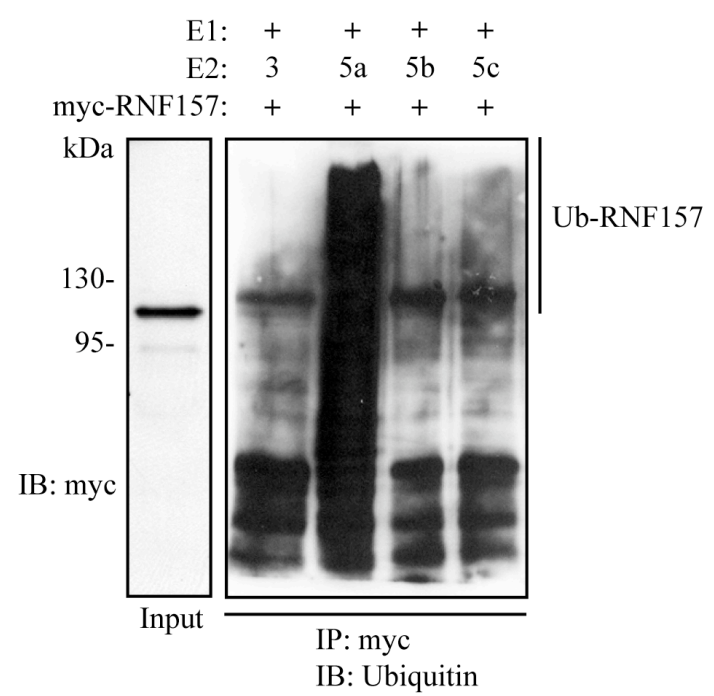

B

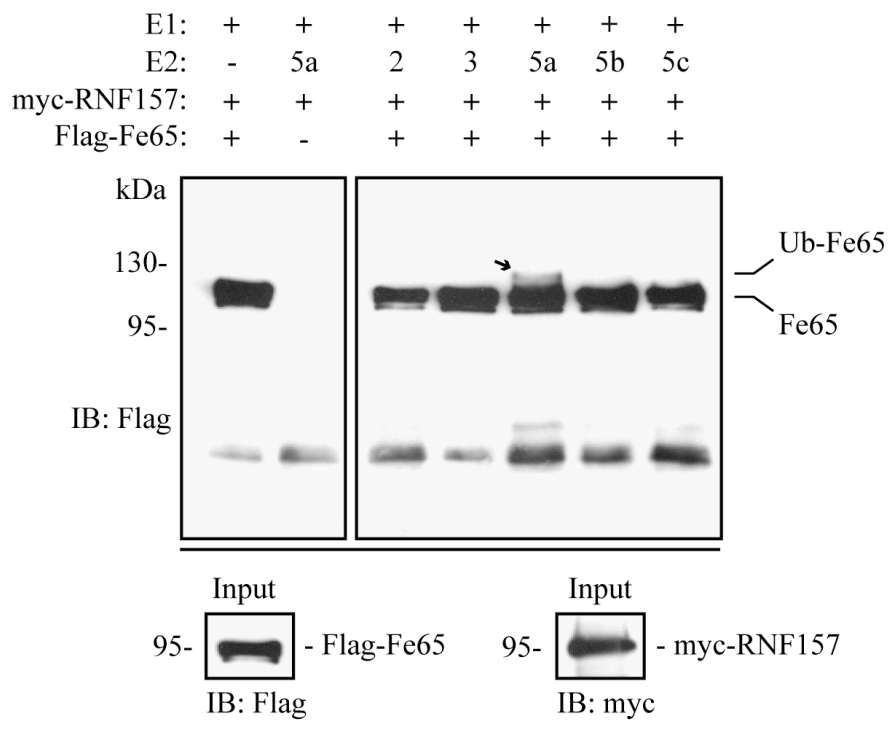

Figure 30. RNF157 ubiquitinates Fe65 in vitro. A) Enzymatic activity of RNF157. Myc-RNF157 was immunoprecipitated from Neuro 2A cells and subjected to in vitro ubiquitination in the presence of ATP, E1, different E2s (UbcH3, UbcH5a, UbcH5b, or UbcH5c) and ubiquitin. Ubiquitinated RNF157 was detected by immunoblotting with an ubiquitin antibody (courtesy of N. Schwedhelm-Domeyer). B) Immunopurified FlagFe65 (as described in Materials and Methods) was subjected to in vitro ubiquitination in the presence of ATP, E1, different E2s (UbcH2, UbcH3, UbcH5a, UbcH5b, or UbcH5c), ubiquitin and immunopurified myc-RNF157 as indicated. Modified Fe65 was detected by immunoblotting using the Flag antibody, to avoid detection of autoubiquitinated RNF157 (courtesy of N. Schwedhelm-Domeyer). IB, immunoblot; IP, immunoprecipitation; Ub, ubiquitin. Arrow indicates Ub-Fe65.

\subsubsection{Knockdown of Fe65 has no effect on dendrite growth in cerebellar granule neurons}

RNF157 regulates dendrite growth and neuronal survival. This raises the question whether Fe65 plays a role in dendrite development. To carry out Fe65 loss-of-function analysis, I generated Fe65 RNAi plasmids that target human, mouse and rat Fe65. The knockdown efficacy of the three different Fe65 RNAi plasmids was tested in heterologous cells. I transfected HEK 293T cells with control vector or the different Fe65 RNAi plasmids together with a plasmid encoding Fe65 and subjected these lysates to immunoblotting four days later. While the Fe65 RNAi plasmid II shows a slight reduction in Fe65 protein level, Fe65 RNAi III exhibits the most efficient knockdown of Fe65 in HEK 293T cells (Figure 31A). Hence, in all further knockdown experiments, I used the Fe65 RNAi III and referred to it as Fe65 RNAi unless stated otherwise. Next, I transfected cerebellar granule neurons at DIV 4 with the control vector or Fe65 RNAi plasmid together with the Bcl- $\mathrm{x}_{\mathrm{L}}$ and GFP expression plasmids and subjected these neurons to immunocytochemistry at DIV 8 using the GFP antibody. Morphological analysis revealed that knockdown of Fe65 has little or no effect on total dendritic length as compared to control-transfected neurons (Figure 31B/C). 
These results suggest that $\mathrm{Fe} 65$ is not involved in the regulation of dendrite morphogenesis.

A

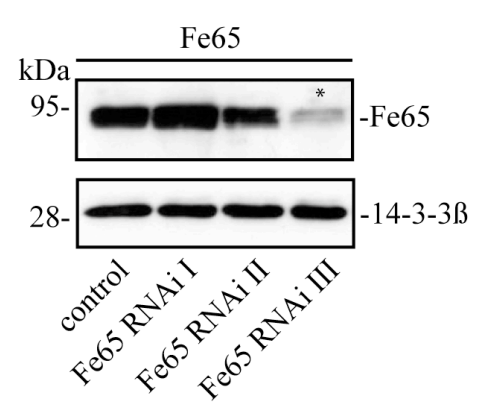

$\mathrm{B}$

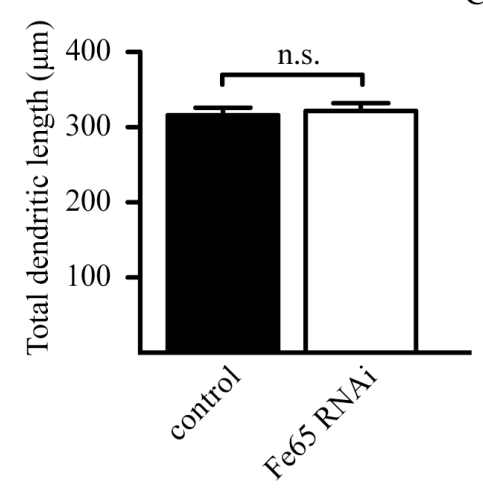

$\mathrm{C}$

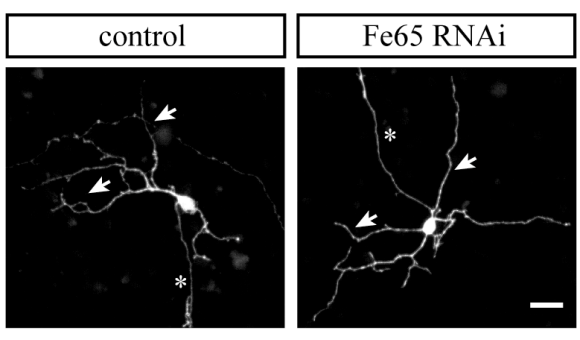

Figure 31. Knockdown of Fe65 has no effect on dendrite morphogenesis. A) Lysates from HEK 293T cells transfected with the expression plasmid encoding myc-Fe65 together with control vector or Fe65 RNAi plasmids I, II or III were immunoblotted with the myc antibody. 14-3-3ß served as loading control. Asterisk indicates functional Fe65 RNAi. B) Cerebellar granule neurons transfected at DIV 4 with the control vector or Fe65 RNAi plasmid together with the Bcl- $\mathrm{x}_{\mathrm{L}}$ and GFP expression plasmids were subjected to immunocytochemistry using the GFP antibody four days later. Knockdown of Fe65 has little or no effect on dendrite growth as compared to control-transfected neurons (n.s, not significant). A total of 217 neurons were analyzed. C) Representative images of neurons transfected with the control vector or Fe65 RNAi plasmid. Asterisks and arrows indicate axons and dendrites, respectively. Scale bar equals $20 \mu \mathrm{m}$.

\subsubsection{Expression of Fe65 induces apoptosis in cerebellar granule neurons}

In further experiments, I analyzed the function of Fe65 in neuronal survival. Given that RNF157's E3 ligase activity is required to promote neuronal survival and that Fe65 is ubiquitinated by RNF157, I reasoned that Fe65 lacking its ubiquitin modification might promote neuronal cell death. Hence, to investigate whether Fe65 acts as a pro-apoptotic protein in neurons, I transfected cerebellar granule neurons at DIV 2 with the control vector or Fe65 expression plasmid together with a plasmid encoding ß-galactosidase and analyzed neuronal viability four days later. Strikingly, I found that overexpression of Fe65 significantly increases apoptosis to 69\% in cerebellar granule neurons (Figure 32). 


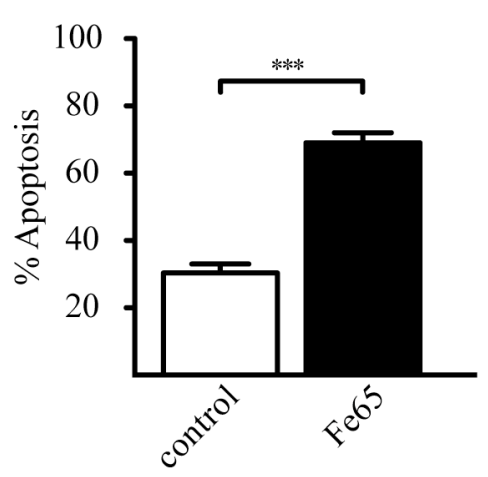

Figure 32. Overexpression of Fe65 induces apoptosis. Cerebellar granule neurons transfected at DIV 2 with the control vector or myc-Fe65 expression plasmid together with a plasmid encoding ß-galactosidase were subjected to immunocytochemistry using the $ß$-galactosidase antibody and the DNA dye bisbenzimide (Hoechst 33258) four days later. Percentage of apoptosis is presented as mean \pm SEM. Overexpression of Fe65 significantly increases apoptosis as compared to control-transfected neurons ( $\mathrm{n}=3$; ANOVA; ${ }^{* * *} \mathrm{p}<$ 0.001). A total of 1037 neurons were analyzed.

To examine if the localization of Fe65 is important to its function in neuronal survival, Fe65 was targeted to the nucleus or the cytoplasm. For this purpose, Dr. Judith Stegmüller fused a nuclear exclusion sequence (NES) or a nuclear localization sequence (NLS) to the N-terminus of pCMV-Fe65. The proper localization of NES-Fe65 and NLS-Fe65 was confirmed in HEK 293T cells as well as in cerebellar granule neurons. NES-Fe65 remains in the cytoplasm whereas NLS-Fe65 localizes to the nucleus (Figure 33A/B). Next, I analyzed the capability of the different Fe65 mutants to induce neuronal apoptosis. I transfected cerebellar granule at DIV 2 with the control vector, wild type Fe65, NES-Fe65 or NLS-Fe65 expression plasmids together with a plasmid encoding ß-galactosidase and subjected these neurons to survival assays four days later. I found that both wild type Fe65 and the NLS-Fe65 mutant trigger apoptosis in cerebellar granule neurons. In contrast, NESFe65, sequestered to the cytoplasm, fails to induce neuronal apoptosis (Figure 33C).

To further elucidate the mechanism of Fe65-induced neuronal apoptosis, I performed structure-function analysis of Fe65 in cerebellar granule neurons. First, I transfected HEK 293T cells and cerebellar granule neurons with plasmids encoding Fe65 wild type or different Fe65 truncation mutants: Fe65aa1-325 (WW domain), Fe65aa326-524 (PTB1 domain), Fe65aa525-711 (PTB2 domain), Fe65aa1-524 (WW+ PTB1 domain) or Fe65aa326711 (PTB1/2 domain) and determined the subcellular localization of these mutants. I found that wild type Fe65 as well as Fe65aa1-325, Fe65aa326-524, Fe65aa1-524 and Fe65aa326-711 are equally distributed between the cytoplasm and the nucleus. Interestingly, Fe65aa525-711 (PTB2 domain) is exclusively present in the nucleus in both HEK 293T cells and cerebellar granule neurons (Figure 34A/B). 
Next, I quantified apoptosis in neurons expressing the aforementioned Fe65 truncation mutants at DIV 6. My results revealed that aside from wild type Fe65, Fe65 aa525711 dramatically increases cell death in granule neurons. Fe65aa1-325, Fe65aa326-524, Fe65aa1-524 and Fe65aa326-711 only show a moderate induction of cell death (Figure 34C). These findings are consistent with my results, which show that Fe65 operates in the nucleus to induce neuronal apoptosis. Moreover, my data indicates that the C-terminal region of Fe65 (aa525-711) harbors a nuclear localization signal.

A
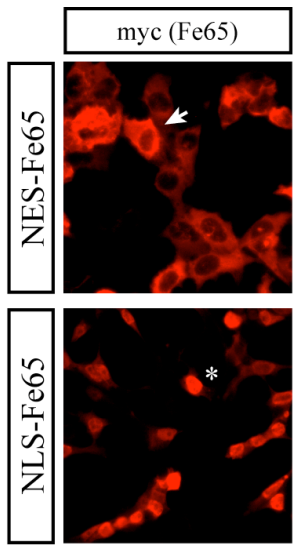

$\mathrm{B}$

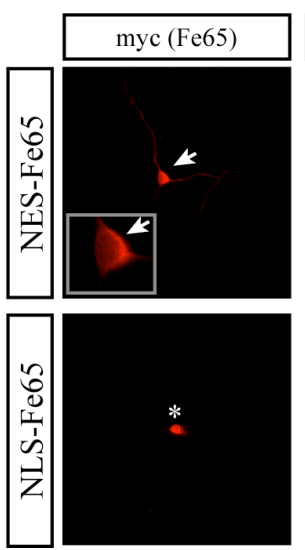

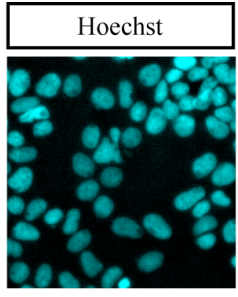
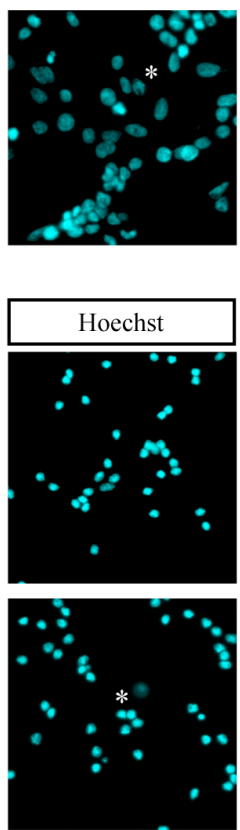
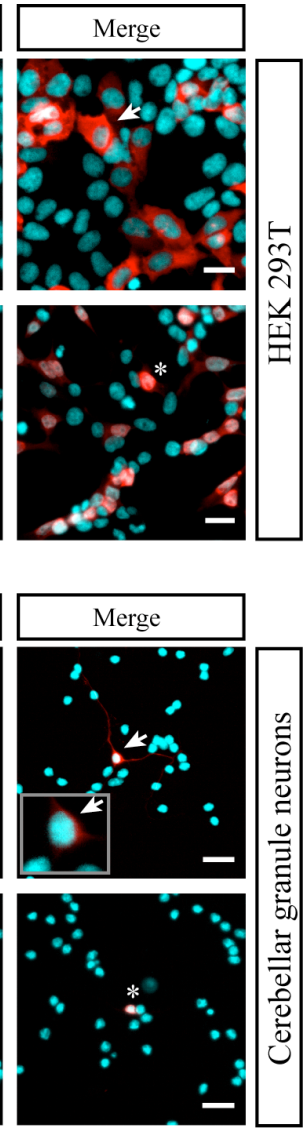

$\mathrm{C}$

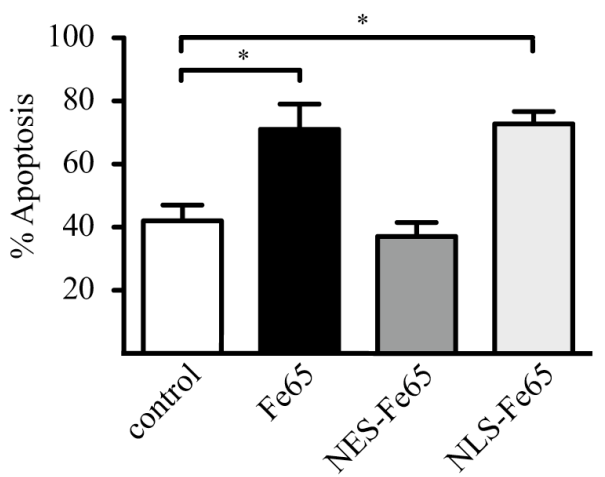

Figure 33. Fe65 operates in the nucleus to induce apoptosis. A/B) HEK 293T cells (A) and cerebellar granule neurons (B) were transfected with the myc-NES-Fe65 and myc-NLS-Fe65 expression plasmids and subjected to immunocytochemistry using the myc antibody and the DNA dye bisbenzimide (Hoechst 33258). Arrows and asterisks indicate cytoplasmic or nuclear localizations, respectively. Insets depict cell bodies indicated by an arrow. Scale bar equals $20 \mu \mathrm{m}$. C) Cerebellar granule neurons transfected at DIV 2 with the control vector, myc-Fe65 wild type, myc-NES-Fe65 or myc-NLS-Fe65 expression plasmids together with a plasmid encoding ß-galactosidase were subjected to immunocytochemistry using the ß-galactosidase antibody and the DNA dye bisbenzimide (Hoechst 33258) four days later. Percentage of apoptosis is presented as mean \pm SEM. Overexpression of Fe65 and NLS-Fe65 but not NES-Fe65 significantly increases apoptosis as compared to control-transfected neurons ( $\mathrm{n}=3$; ANOVA; $\left.{ }^{*} \mathrm{p}<0.05\right)$. A total of 1834 neurons were analyzed. 
A

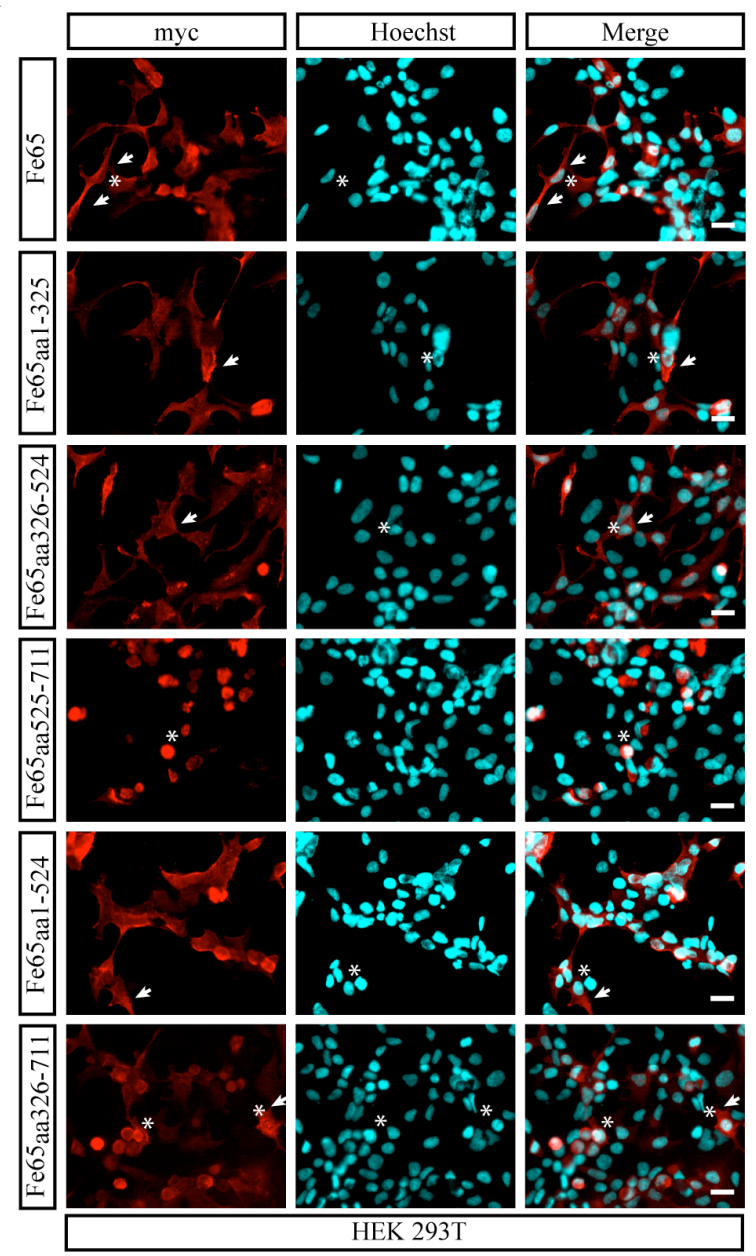

B

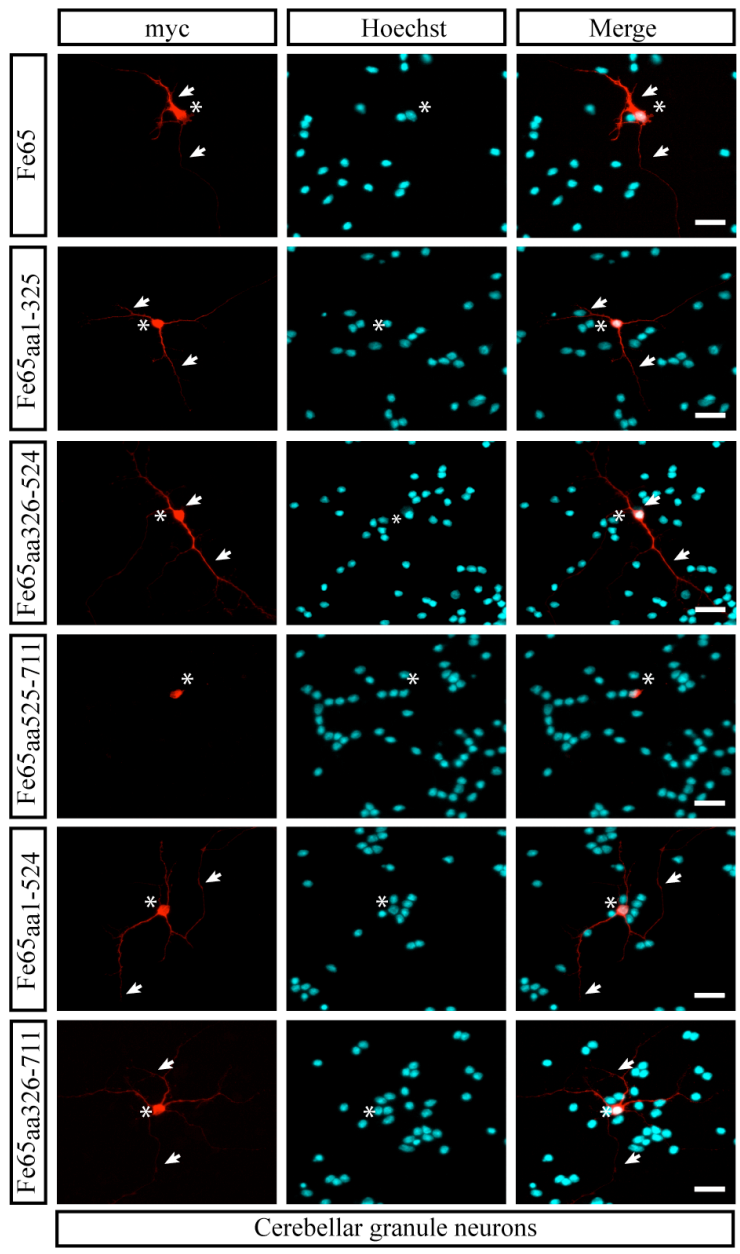

$\mathrm{C}$

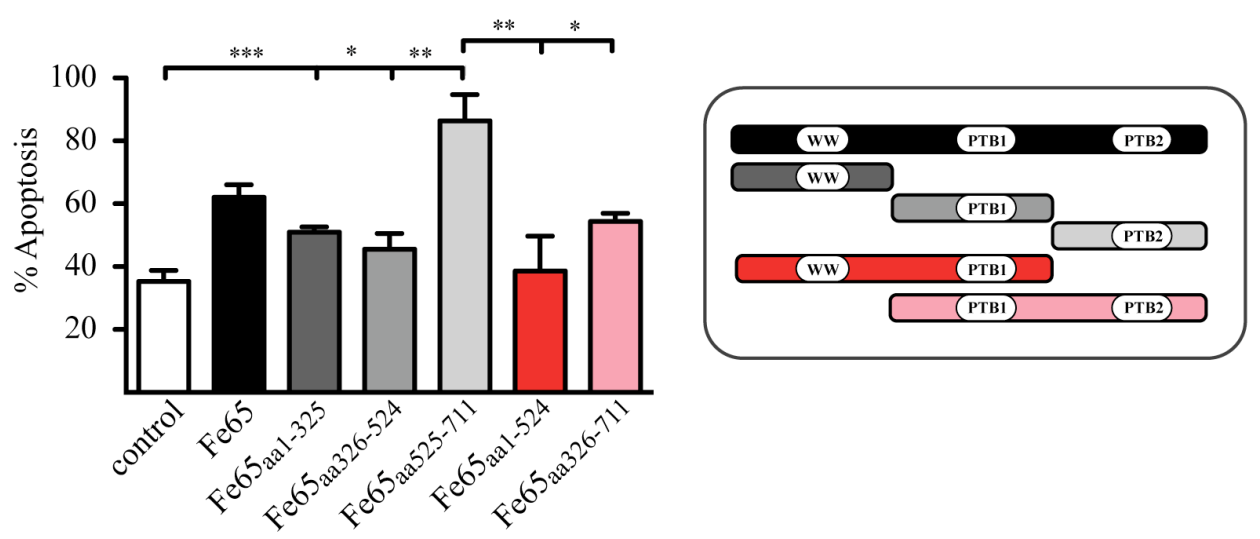

Figure 34. Fe65 aa525-711 (PTB2) translocates to the nucleus to initiate apoptosis. A/B) HEK 293T cells (A) and cerebellar granule neurons (B) transfected with the myc-Fe65 wild type, myc-Fe65aa1-325 (WW domain), myc-Fe65 aa326-524 (PTB1 domain), myc-Fe65 aa525-711 (PTB2 domain), myc-Fe65aa1-524 (WW+ PTB1 domain) or myc-Fe65 aa326-711 (PTB1/2 domain) expression plasmids were subjected to immunocytochemistry using the myc antibody and the DNA dye bisbenzimide (Hoechst 33258). Arrows and asterisks indicate cytoplasmic/axonal/dendritic or nuclear localizations, respectively. Scale bar equals $20 \mu \mathrm{m}$. C) (Left) Cerebellar granule neurons transfected at DIV 2 with the control vector, myc-Fe65, myc-Fe65aa1-325 (WW domain), myc-Fe65 aа326-524 (PTB1 domain), myc-Fe65 aa525-711 $_{\text {(PTB2 domain), myc-Fe65 aa1-524 }}$ (WW+PTB1 domain) or myc-Fe65 aа326-711 (PTB1/2 domain) expression plasmids together with a plasmid encoding ßgalactosidase were subjected to immunocytochemistry using the ß-galactosidase antibody and the DNA dye bisbenzimide (Hoechst 33258) four days later. Percentage of apoptosis is presented as mean \pm SEM. 
Overexpression of Fe65 aa525-711 dramatically increases apoptosis as compared to control-transfected neurons $\left(\mathrm{n}=3\right.$; ANOVA; $\left.{ }^{*} \mathrm{p}<0.05 ;{ }^{* *} \mathrm{p}<0.01 ;{ }^{* * *} \mathrm{p}<0.001\right)$. A total of 3203 neurons were analyzed. (Right) Color of mutants match columns in survival assay.

\subsubsection{Knockdown of Fe65 reduces starvation-induced apoptosis in cerebellar granule neurons}

Given that Fe65 acts as a pro-apoptotic protein, I reasoned that knockdown of Fe65 under apoptotic stimuli may attenuate neuronal cell death. Hence, I transfected cerebellar granule neurons at DIV 2 with the control vector or Fe65 RNAi plasmid together with a plasmid encoding ß-galactosidase. At DIV 5, conditioned media was replaced with BME 112 starvation media (= $\mathrm{KCl} /$ serum withdrawal) for $18 \mathrm{hr}$. Neurons were subsequently fixed and immunostained with the ß-galactosidase antibody. As expected, starvation leads to a high rate of apoptosis in control neurons. Strikingly, apoptosis is significantly reduced by 50\% in Fe65 RNAi-transfected neurons (Figure 35). These results suggest that Fe65 promotes apoptosis in cerebellar granule neurons.

A

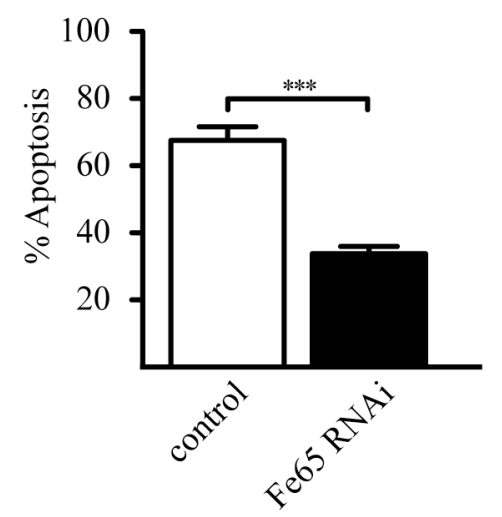

B

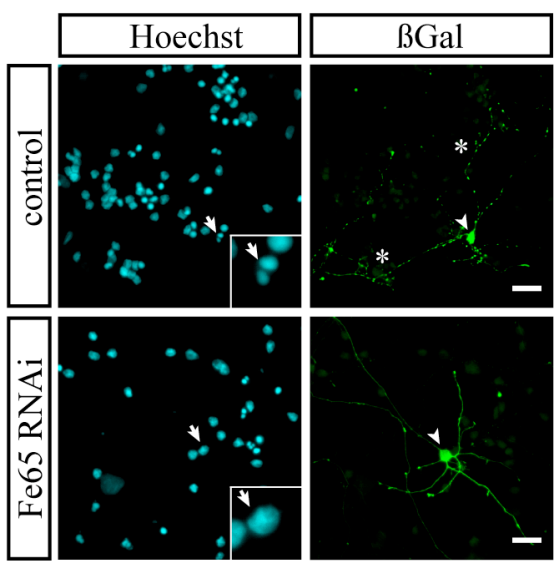

Figure 35. Knockdown of Fe65 reduces starvation-induced apoptosis. A) Cerebellar granule neurons were transfected at DIV 2 with the control vector or Fe65 RNAi plasmid together with a plasmid encoding ßgalactosidase. Three days later, neurons were starved in $\mathrm{KCl}$ and serum-free media for $18 \mathrm{hr}$ and subjected to immunocytochemistry using the ß-galactosidase ( $\mathrm{BGal}$ ) antibody and the DNA dye bisbenzimide (Hoechst 33258). Percentage of apoptosis is presented as mean \pm SEM. Knockdown of Fe65 significantly decreases apoptosis as compared to control-transfected neurons $\left(\mathrm{n}=4\right.$; Student's t-test; $\left.{ }^{* * *} \mathrm{p}<0.001\right)$. A total of 2476 neurons were analyzed. B) Representative images of neurons transfected with the control vector or Fe65 RNAi plasmid. Arrows, arrowheads and asterisks indicate nuclei, soma or apoptotic bodies of transfected neurons, respectively. Insets depict nuclei indicated with an arrow. Scale bar equals $20 \mu \mathrm{m}$. 


\subsubsection{RNF157 knockdown-induced increase in neuronal apoptosis can be} alleviated by simultaneous knockdown of the death receptor 6 (DR6)

Neuronal apoptosis can be induced by intracellular proteins or extrinsic factors, which bind to death domain (DD) containing cell surface receptors of the tumor necrosis factor (TNF) receptor superfamily. One of those receptors is the death receptor 6 (DR6). Aside from heart and placenta, DR6 is highly expressed in the brain (Bhardwaj \& Aggarwal, 2003). Interestingly, upon trophic deprivation in vitro, binding of an extracellular fragment of the amyloid precursor protein (APP) triggers the activation of DR6. The activated receptor-ligand complex then regulates neuronal cell body death in a caspase-3 and axonal degeneration in a caspase-6-dependent manner (Nikolaev et al, 2009). In my study, I identified the APP adaptor protein Fe65 as a novel interactor of RNF157. Fe65 regulates APP processing and trafficking as well as the balance between amyloidogenic and nonamyloidogenic cleavage of APP

Owing to the finding that RNF157 overexpression reduces neuronal cell death upon serum deprivation and that RNF157 is interacting with Fe65, I asked whether there is a functional relationship between RNF157 and DR6 in neuronal survival.

To answer this question, I generated three different DR6 RNAi plasmids and tested the efficacy to knockdown exogenous DR6 in HEK 293T cells. Immunoblotting of the lysates revealed that the DR6 RNAi III efficiently reduces the level of exogenous DR6 in heterologous cells (Figure 36A). Next, I transfected cerebellar granule neurons at DIV 2 with the control vectors, the RNF157 RNAi plasmid with control vector, the DR6 RNAi III plasmid with control vector, or both the RNF157 and DR6 RNAi III plasmid together with a plasmid encoding ß-galactosidase and carried out survival assays at DIV 6. While RNF157 knockdown triggers neuronal apoptosis, DR6 knockdown alone has little or no effect on neuronal survival. Strikingly, simultaneous knockdown of RNF157 and DR6 reverses the RNF157 knockdown-induced increase in cell death, resulting in 33,7\% of apoptotic neurons that is comparable to control-transfected neurons (Figure 36B). These results suggest that DR6 operates downstream of RNF157 in the control of neuronal survival. 
A

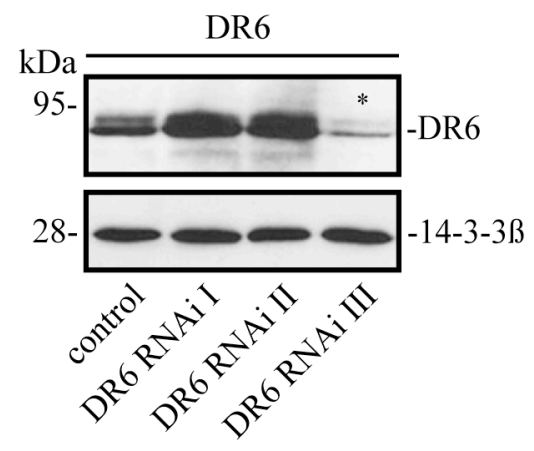

B

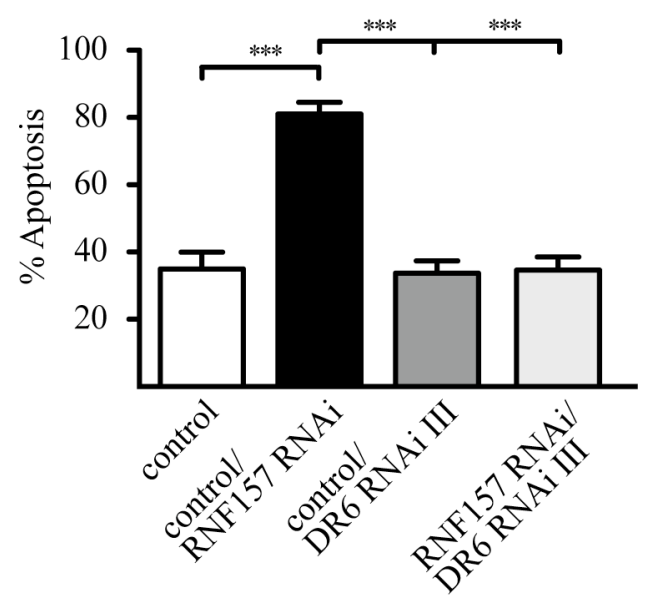

Figure 36. RNF157 acts in a linear pathway with DR6 in the control of neuronal survival. A) Lysates from HEK 293T cells transfected with a plasmid encoding myc-DR6 together with the control vector, DR6 RNAi plasmids I, II or III were immunoblotted with the myc antibody. 14-3-3ß served as loading control. Asterisk indicates functional DR6 RNAi. B) Cerebellar granule neurons transfected at DIV 2 with the control vectors, RNF157 RNAi plasmid with control vector, DR6 RNAi III plasmid with control vector, or both RNF157 and DR6 RNAi III plasmids together with a plasmid encoding ß-galactosidase were subjected to immunocytochemistry using the ß-galactosidase antibody and the DNA dye bisbenzimide (Hoechst 33258) four days later. Percentage of apoptosis is presented as mean \pm SEM. Knockdown of both RNF157 and DR6 significantly decreases apoptosis as compared to RNF157 RNAi-transfected neurons $(n=3$; ANOVA; $\left.{ }^{* * *} \mathrm{p}<0.001\right)$. A total of 2502 neurons were analyzed.

\subsubsection{Fe65 acts downstream of RNF157 in a linear pathway in the control of neuronal survival}

To analyze whether RNF157 and Fe65 act in a linear pathway to regulate neuronal survival, I performed epistatic analysis. I transfected cerebellar granule neurons at DIV 2 with the control vectors, RNF157 RNAi plasmid with control vector, or both RNF157 RNAi and Fe65 RNAi plasmids together with a plasmid encoding ß-galactosidase and quantified neuronal apoptosis four days later. While knockdown of RNF157 significantly increases apoptosis as compared to control-transfected neurons, simultaneous knockdown of RNF157 and Fe65 leads to a significant reduction of cell death that is similar to the apoptotic rate of control-transfected neurons (Figure 37). My results suggest that Fe65 knockdown suppresses the RNF157 knockdown-induced increase in apoptosis and support the notion that Fe65 acts downstream of RNF157 in the control of neuronal survival. 


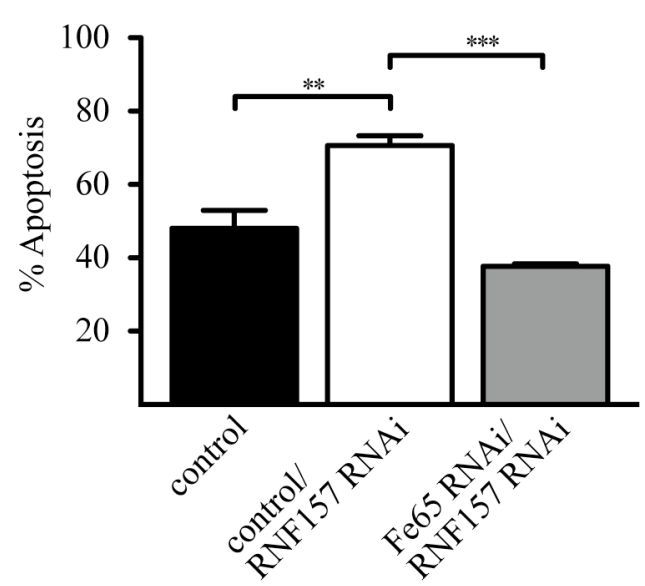

Figure 37. Fe65 acts downstream of RNF157 in the control of neuronal survival. Cerebellar granule neurons transfected at DIV 2 with the control vector, RNF157 RNAi plasmid with control vector, or both RNF157 and Fe65 RNAi plasmids together with a plasmid encoding ß-galactosidase were subjected to immunocytochemistry using the ß-galactosidase antibody and the DNA dye bisbenzimide (Hoechst 33258) four days later. Percentage of apoptosis is presented as mean \pm SEM. Knockdown of both RNF157 and Fe65 significantly decreases apoptosis as compared to RNF157 RNAi-transfected neurons (n=3; ANOVA; ${ }^{* *} \mathrm{p}<0.01$; $\left.{ }^{* * *} \mathrm{p}<0.001\right)$. A total of 1765 neurons were analyzed.

\subsection{Characterization of a mouse model lacking RNF157 function}

\subsubsection{Generation of RNF157 gene trap mice}

In order to understand the role of RNF157 in the context of mammalian brain development, we took a gene trap approach to disrupt RNF157 protein expression at the systemic level in mice. Mouse RNF157 gene is located on chromosome 11 with 19 exons and 18 introns. The purchased ES cell clone contains a gene trap vector with a $B G e o$ cassette inserted into the second intron of the RNF157 gene, which produces a truncated fusion mRNA only encoding the first 2 exons of RNF157, which do not encode any functional domains (Figure 38A). The ES cells were injected into FVB/N blastocysts to obtain chimeric animals, which were then bred with $\mathrm{C57B} / 6 \mathrm{~N}$ mice to obtain germline transmission. To verify offspring carrying the RNF157 gene trap cassette, I performed PCR genotyping (Figure 38B).

A

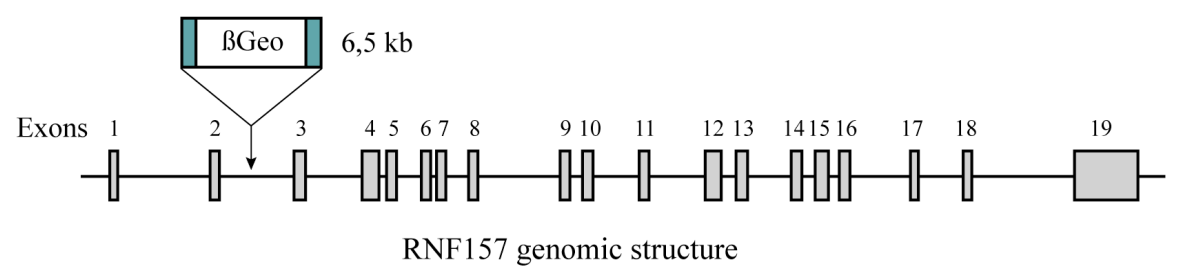

B

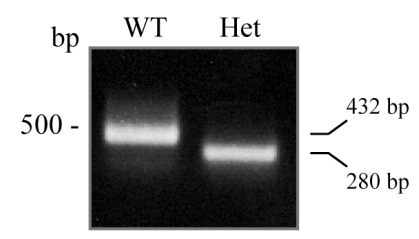

Figure 38. Generation of RNF157 gene trap mice. A) Genomic structure of the mouse RNF157 gene. 19 exons and 18 introns are shown, along with the insertion site for the targeting vector. The target vector was inserted into the second intron, as indicated in the schematic. B) PCR-based genotyping. Genomic DNA was 
isolated from ES cells and subjected to PCR amplification. Shown are two fragments with sizes of $432 \mathrm{bp}$ (WT) and 280 bp (mutant). WT, wild type; Het, heterozygous.

\subsubsection{Analysis of RNF157 transgenic animals}

To confirm the reduction of RNF157 in RNF157 heterozygous (Het) animals, Shih-Ju Lee prepared lysates from the adult cortex of wild type and RNF157 heterozygous mice and subjected them to immunoblotting using the ß-galactosidase antibody. She found that ß-galactosidase is expressed in RNF157 Het but not in wild type littermates (Figure 39A). Furthermore, immunoblotting of the aforementioned lysates using the RNF157 antibody showed a reduction of RNF157 protein levels in the RNF157 Het animals as compared to wild type littermates (Figure 39B). These results are consistent with the genotypes defined by PCR-reaction.

A

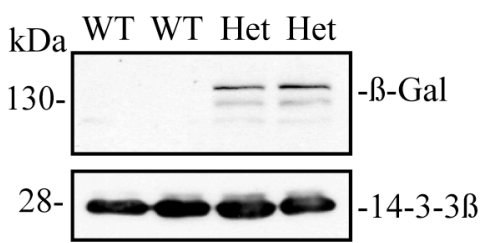

$\mathrm{B}$

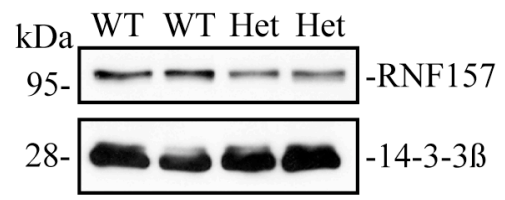

Figure 39. Analysis of RNF157 transgenic animals. A/B) Cortical lysates of adult RNF157 wild type and heterozygous animals were immunoblotted using the ß-galactosidase (ß-Gal) or RNF157 antibodies, respectively. 14-3-3ß served as loading control. WT, wild type; Het, heterozygous.

Next, I crossed RNF157 Het mice to obtain RNF157 homozygous animals. Strikingly, among 166 genotyped offspring, none was homozygous for the gene-trapped allele. The ratio of wild type mice to heterozygous mice was around 1:3 (Table 2). This raised the question if RNF157 null embryos die during embryonic development. To answer this, I analyzed embryos at E7 and E10 and found that so far neither at E7 nor at E10 viable homozygous RNF157 embryos were detectable. These results suggest that disruption of RNF157 function causes early embryonic lethality of RNF157 homozygous embryos.

Table 2. Genotypes of RNF157 mutant mice or embryos.

\begin{tabular}{|c|c|c|c|c|}
\hline Age & RNF157 WT & RNF157 Het & RNF157 Hom & Total \\
\hline Postnatal & $29(25 \%)$ & $87(75 \%)$ & 0 & 116 \\
\hline E10 & $3(33.3 \%)$ & $6(66.6 \%)$ & 0 & 9 \\
\hline E7 & $13(31.7 \%)$ & $28(68.3 \%)$ & 0 & 41 \\
\hline
\end{tabular}


In summary, these results uncover an essential function for RNF157 during embryonic development that is accompanied by the survival-promoting function of RNF157 in vitro.

\subsubsection{Histologic analysis of RNF157 heterozygous mice}

To confirm the spatial expression pattern of RNF157 protein obtained by immunoblotting, Shih-Ju Lee analyzed the enzymatic $\beta$-galactosidase activity in sagittal vibratome sections of RNF157 heterozygous adult mice brains. She found enzymatic $\beta$ galactosidase activity in cerebellum, midbrain, thalamus, hypothalamus, caudate putamen, anterior olfactory nucleus with high levels in cortex and hippocampus (Figure 40). Since the endogenous RNF157 promoter drives the expression of the ßGeo cassette, these results confirm the spatial expression of RNF157 in cortex, cerebellum and hippocampus obtained by immunoblotting using the RNF157 antibody.

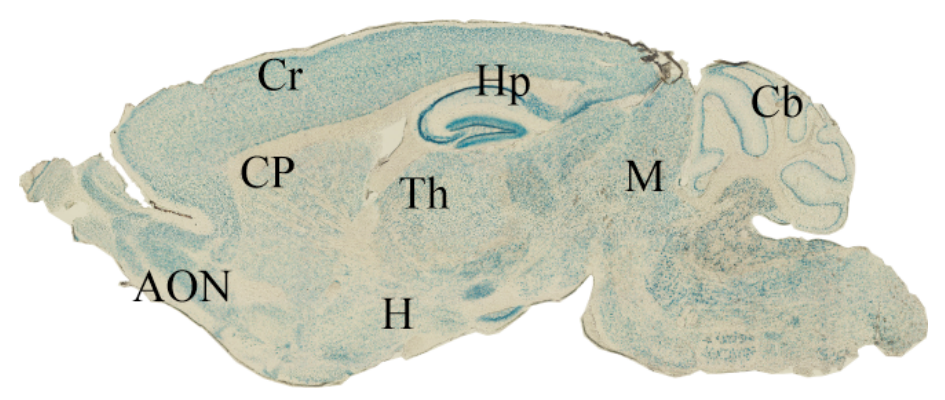

Figure 40. X-Gal staining of sagittal brain section of RNF157 heterozygous mouse. Blue signals represent the RNF157 promoter activity. C, cerebellum; M, midbrain; Th, thalamus; H, hypothalamus; CP, caudate putamen; AON, anterior olfactory; Cr, cortex; Hp, hippocampus. (Courtesy of S.J. Lee)

Next, I analyzed whether RNF157 Het animals show an increase in astrocytosis, which was reported for Mgrn1 null mice (Bagher et al, 2006). To answer this, paraffin sections of 12-week-old RNF157 wild type and heterozygous littermates $(n=2)$ were stained with GFAP. I found no significant difference in the number of GFAP positive cells between wild type and RNF157 Het animals (Figure 41). 

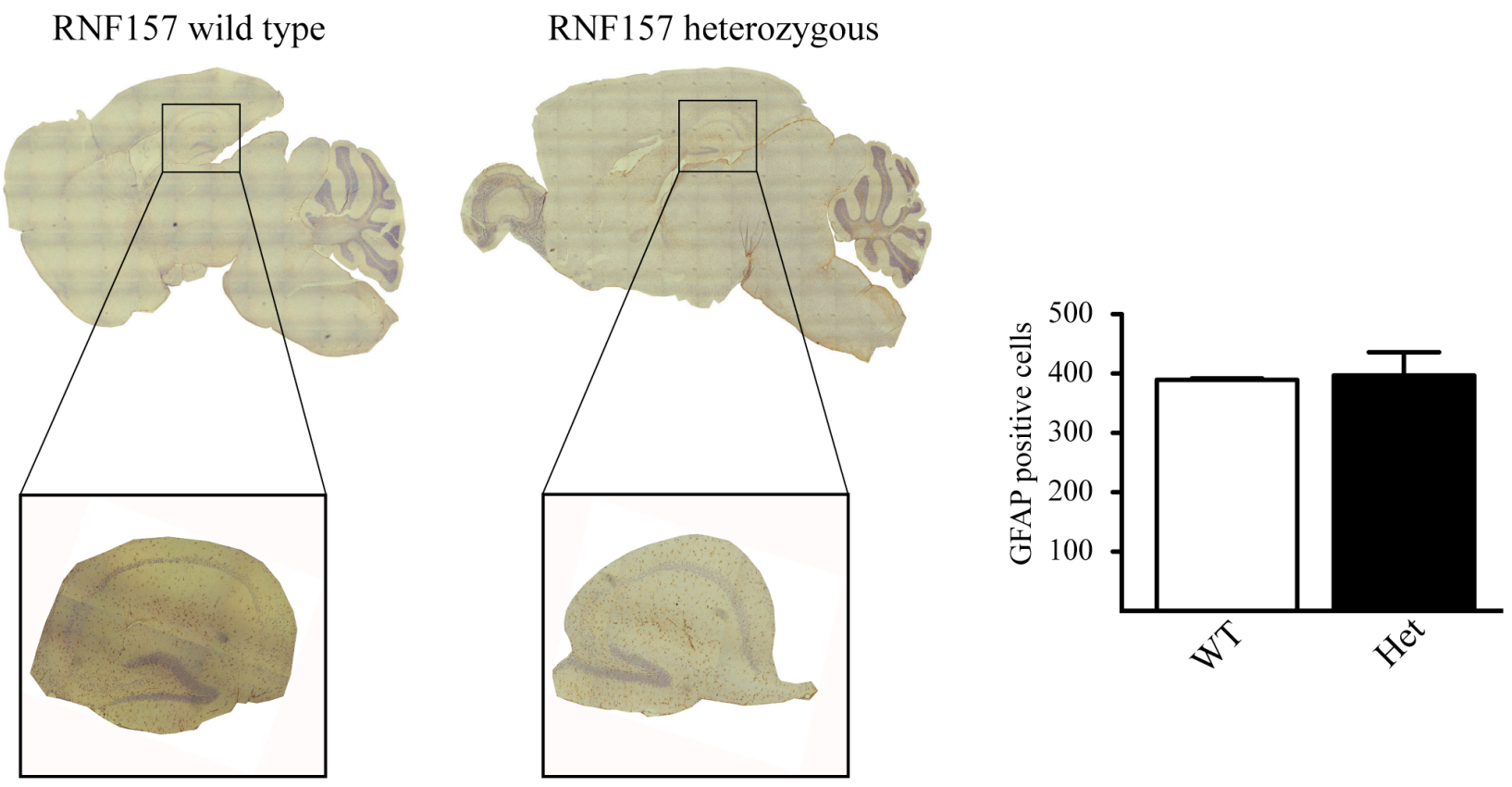

Figure 41. Histologic analysis of RNF157 heterozygous mice. (left) Representative images of sagittal brain sections of 12 weeks old RNF157 wild type (WT) and heterozygous (Het) littermates stained with the GFAP antibody and haematoxylin. Insets depict hippocampi. (right) Quantification of GFAP positive cells in the hippocampus ( $\mathrm{n}=2)$. A total of 1573 cells were analyzed. 


\section{Discussion}

Apoptosis, a cell-intrinsic suicide program, is a highly orchestrated process that is indispensable to normal development of the central nervous system, while dysregulation of apoptosis contributes to neuronal loss observed in neurological injury and disease. Growing evidence demonstrates that the post-translational modification of proteins by ubiquitination regulates key components of the cell death machinery (Vucic et al, 2011; Whatley et al, 2008). Here, ubiquitination can either regulate the turnover of proteins by proteasome-dependent degradation or bring about important functional modifications (Schnell \& Hicke, 2003; Vucic et al, 2011). The link between perturbations in ubiquitination and neurodegenerative disorders was supported by the discoveries that loss of the E3 ubiquitin ligases Mahogunin RING finger-1 (Mgrn1) and LISTERIN results in neurodegeneration (Chu et al, 2009; He et al, 2003). However, there is little understanding of ubiquitination-dependent molecular mechanisms underlying both developmentally regulated as well as disease-related neuronal apoptosis. In the present study, I have identified the RING E3 ligase RNF157 as an important regulator of neuronal survival. In addition, morphological analyses revealed that RNF157 promotes dendrite morphogenesis and complexity in an E3 ligase activity-independent manner. Furthermore, I have identified the adaptor protein Fe65 as a novel interactor of RNF157 that operates in the nucleus to initiate neuronal apoptosis. In addition, Fe65 is a key target of RNF157 and subject to nondegradative ubiquitination. Consistent with these results, epistasis analysis revealed that Fe65 acts downstream of RNF157 in the control of neuronal survival. In conclusion, my study uncovers an RNF157-Fe65 pathway that regulates neuronal survival in vitro and thereby contributes to the understanding of E3 ligases in neuronal survival and apoptosis. In addition, my work led to the generation of an RNF157 knockout mouse model, which indicates the importance of RNF157 during embryonic development.

\subsection{RNF157 is a brain-dominant protein that is expressed throughout development}

The RING finger protein RNF157 is a novel uncharacterized RING E3 ligase. In my study, I found that RNF157 is a brain-dominant protein, which is expressed throughout 
development in cortex, cerebellum and hippocampus. Endogenous RNF157 localizes to the cytoplasm and is absent from the nucleus in neurons. After neuronal polarization, RNF157 is present in the axon and primary dendrites. Interestingly, although RNF157 mRNA is detectable in heart, lung and kidney, RNF157 protein however is merely detectable in the brain. This suggests that the stability of the RNF157 mRNA varies between different cell types of the body, which leads to the dominant expression of RNF157 in the brain. In 2009, Jiao and colleagues identified RNF157 (Mgrn2) as a homologue of the E3 ligase Mgrn1, since they are 69\% identical and share high similarities in the N-terminal region. Although both proteins harbor a conserved RING domain, RNF157 lacks the PSAP tetrapeptide motif, which is essential for the interaction of Mgrn1 with TSG101, a component of the ESCRT (endosomal sorting complex required for transport) machinery (Kim et al, 2007a). Furthermore, the expression of Mgrn1 is not restricted to the brain, with high expression in heart, kidney and liver (He et al, 2003). At the subcellular level, endogenous Mgrn1 is localized in the nucleus and the cytoplasm, where it shows a punctuated staining pattern (Kim et al, 2007a). Hence, although RNF157 is a homologue of Mgrn1, my results demonstrate that the expression and subcellular localization of RNF157 is different from Mgrn1 and that RNF157 may have a specific function in the brain.

However, the expression of RNF157 in other regions of the brain as well as during embryonic development requires further analysis. In addition, it will be interesting to examine the expression and localization of RNF157 in astrocytes and oligodendrocytes, since RNF157 mRNA is present in cultured astrocytes and in the oligodendrocyte cell line OLI-neu.

The Ensembl project predicts three additional isoforms of RNF157, which are smaller in size as they differ in their exon combination. Analyses of their spatial and temporal expression as well as their subcellular localization would provide a more detailed view about RNF157 in the brain. In preliminary immunocytochemical experiments, I found that a second RNF157 antibody, which is recognizing a different region within the RNF157 protein, displays a completely different staining pattern of RNF157 in cerebellar granule neurons (data not shown). My results imply that this antibody detects a smaller RNF157 isoform. Accordingly, I hypothesize that the different RNF157 isoforms may differ in their subcellular localization and thus may perform different functions in neurons. 


\subsection{RNF157 promotes neuronal survival in vitro}

Increasing evidence identified RING E3 ligases in neuronal survival and apoptosis, while their specific target proteins remain mostly unidentified. In the present study, I uncovered that the RING E3 ligase RNF157 is an important regulator of neuronal survival. RNF157 loss-of-function dramatically increases apoptosis in cerebellar granule and hippocampal neurons, which could be reversed by the expression of an RNAi-resistant form of RNF157, underscoring the specificity of the RNF157 RNAi-mediated phenotype. Moreover, the RNF157 knockdown-induced increase of neuronal cell death was suppressed by simultaneous expression of the pro-survival Bcl-2 family member Bcl- $\mathrm{x}_{\mathrm{L}}$, suggesting that knockdown of RNF157 leads to activation of the apoptotic machinery. Strikingly, in gain-of-function experiments, wild type RNF157 but not RNF157 $\Delta$ RING increases neuronal survival under stress conditions. This indicates that the E3 ligase activity of RNF157 is required for the regulation of neuronal survival. In summary, my results suggest that RNF157 regulates the survival of neurons in a ligase-dependent manner. In future studies, it will be interesting to determine if the function of RNF157 is restricted to a specific developmental time window or required during the whole lifespan of a neuron. In addition, it will be interesting to investigate if RNF157 exerts a similar function in oligodendrocytes and astrocytes.

Interestingly, also Mgrn1 is important for neuronal survival in the mammalian brain. He and colleagues reported that Mgrn1 null mice develop progressive spongiform neurodegeneration (He et al, 2003). The first pathological changes appear at 2 month of age in the CA3 region of the hippocampus and expand to other brain regions at later ages. The Mgrn1 null brain is characterized by vacuolation of the gray matter and neuronal loss in the cerebral cortex, hippocampus, thalamus and brain stem (He et al, 2003). Thus, both RNF157 and Mgrn1 promote neuronal survival. However, since the Mgrn1 null mouse displays severe defects, Mgrn1 and RNF157 are not functionally redundant. Moreover, while Mgrn1 was shown to interact with TSG101, a key component of the endosomalsorting complex, RNF157 does not associate with TSG101 (Jiao et al, 2009). Hence, these results suggest that while Mgrn1 and RNF157 are important for neuronal survival, they control different signaling pathways.

In addition to Mgrn1 and RNF157, recent studies implicate other RING E3 ligases in the regulation of neuronal survival and apoptosis (see chapter 1.3.1.2). Nateri and colleagues reported that the RING E3 ligase SCFFbw7, which mediates the ubiquitination and 
subsequent degradation of phosphorylated c-Jun, has an anti-apoptotic function in neurons. Fbw7 loss-of-function leads to accumulation of phosphorylated c-Jun and neuronal cell death, which could be reduced by overexpression of the JNK inhibitor JIP-1 (Nateri et al, 2004). Furthermore, Sahara and colleagues demonstrated that the U-box E3 ligase carboxyl terminus of Hsp70-interacting protein (CHIP) interacts with the molecular chaperone Hsc90 to ameliorate tau aggregation, whereby the levels of CHIP are inverse proportional to the levels of aggregated tau protein in Alzheimer's disease (AD) brains (Sahara et al, 2005). In addition, Shimura and colleagues showed that the CHIP-Hsc70 complex ubiquitinates and degrades phosphorylated tau in vitro thereby attenuating phosphorylated tau-induced cell death (Shimura et al, 2004). In contrast to the protective effects of RING E3 ligases, Liu and colleagues reported that the RING E3 ligase RNF182, which is upregulated in the brains of AD patients, induces apoptosis. While overexpression of RNF182 in Neuro 2A cells increases cell death, RNAi-mediated knockdown of RNF182 reduces apoptosis induced by oxygen and glucose deprivation (OGD). These results implicate a role for RNF182 in the initiation of apoptosis (Liu et al, 2008). Another example is the RING E3 ligase Trim11, which was shown to bind and destabilize Humanin, a neuroprotective peptide, thereby antagonizing the toxic effects in $\mathrm{AD}$ that are induced by AD-linked mutant genes or the Aß peptide (Hashimoto et al, 2001a; Hashimoto et al, 2001b; Hashimoto et al, 2001c). Finally, Chu and colleagues found in a genome-wide Nethyl-N-nitrosourea (ENU) mutagenesis screen, that mutations in the lister gene lead to early-onset and progression of neurodegeneration and motor dysfunctions. Although lister encodes a RING E3 ligase, LISTERIN, that is ubiquitously expressed, it is motor neurons and neuronal processes in the brain stem and spinal cord that are primarily affected in lister mutant mice. In addition, targeted gene trap insertion revealed that LISTERIN null mice are embryonic lethal (Chu et al, 2009). Herewith the authors provide a novel link between perturbations in ubiquitination and neurodegenerative disorders.

In summary, the aforementioned examples demonstrate the importance of the ubiquitin-proteasome system (UPS) in neuronal viability and that failure of the UPS can lead to a widespread spectrum of neurological disorders (Vucic et al, 2011; Whatley et al, 2008). In this context, my results identify the E3 ligase RNF157 as an important regulator of neuronal survival and thereby contribute to the bigger picture of the UPS as a crucial regulator of neuronal survival and apoptosis. Accordingly, elucidation of the RNF157- 
mediated regulation of neuronal survival will improve our understanding of the UPS in neuronal viability.

\subsection{RNF157 is a novel regulator of dendrite growth independently of its E3 ligase activity}

Dendrite development is a tightly regulated and highly orchestrated process. Establishment of the various neuronal type-specific dendritic arborization patterns is a hallmark of the neuron and essential for proper neuronal circuit formation. Growing evidence demonstrates the importance of cell-intrinsic signaling pathways in the control of dendrite morphogenesis, but the underlying mechanisms remain poorly understood (Jan \& Jan, 2003; Urbanska et al, 2008; Wong \& Ghosh, 2002). Over the last decade, an increasing number of RING E3 ligases have been reported to control dendrite growth and arborization. One of them is the E3 ligase Cul7 Fbxw8, which regulates Golgi integrity and dendrite patterning in cerebellar granule neurons in vitro by ubiquitination of the Golgi protein Grasp65 (Litterman et al, 2011). In addition, Kim and colleagues showed that the E3 ligase APC together with its key regulatory subunit Cdc20 controls dendrite morphogenesis in postmitotic neurons by ubiquitination and proteasomal degradation of the transcription factor Id1 (Kim et al, 2009). In the present study, I have identified a novel E3 ligase, RNF157, involved in the regulation of dendrite morphogenesis in postmitotic neurons in vitro. RNF157 loss-of-function significantly reduces dendritic length and complexity in cerebellar granule neurons. Furthermore, RNF157 gain-of-function significantly increases dendrite growth and complexity. In addition, RNF157 loss-offunction in hippocampal neurons reduces total dendritic length by $85 \%$, suggesting that the function of RNF157 represents a generalizable mechanism to regulate dendrite growth in neurons. Interestingly, RNF157 RNAi or RNF157 overexpression has little or no effect on axonal length. Thus, my results demonstrate that RNF157 specifically controls the generation of dendrites but not axons in different neuronal cell types. In addition, my results suggest that RNF157 contributes to the maintenance of dendritic arborization.

In contrast to the wealth of knowledge regarding axonal and dendritic morphogenesis, relatively little is known about the signaling events, which regulate and sustain synapse and dendrite maintenance. In the mammalian brain, increasing evidence suggests that once a neuron has formed its dendritic arbor, the amount of synaptic inputs from afferent neurons stabilizes the existing branches and suppresses surplus branch 
growth (Chen \& Nedivi, 2010; Cline, 2001; Grueber \& Sagasti, 2010). Moreover, various studies have identified that activity-dependent dendrite maintenance is mediated by calcium-dependent signaling that regulates various downstream signaling cascades including $\mathrm{Ca}^{2+} /$ calmodulin-dependent protein kinases (CaMKs) and Rho family GTPases (Gaudilliere et al, 2004; Lee et al, 2000). Conversely, various activity-independent factors have been identified in the regulation of dendrite maintenance (Hoogenraad et al, 2005; Wills et al, 2012). For example, direct interaction of the synapse scaffold protein PSD-95 with the microtubule end-binding protein EB3 locally suppresses dendrite branching (Charych et al, 2006; Sweet et al, 2011). Moreover, epigenetic mechanisms and dendriteextracellular matrix (ECM) interactions were assumed to regulate dendrite stability and maintenance (Emoto, 2012). In contrast, defects in dendrite maintenance are often associated with neurodevelopmental disorders. Reduced dendritic complexity is observed in patients with autism spectrum disorders, schizophrenia and major depressive disorders (MDD) (Broadbelt et al, 2002; Mukaetova-Ladinska et al, 2004; Raymond et al, 1996). Understanding of the molecular pathways involved in dendrite growth and maintenance will help to identify the pathological mechanisms involved in these neurodegenerative disorders. Hence, in view of these observations, it will be essential to define how RNF157 promotes dendrite morphogenesis in neurons. Notably, my results suggest that RNF157 regulates dendrite growth in an E3 ligase activity-independent manner as overexpression of RNF157 as well as RNF157 $\triangle$ RING significantly increases dendritic length in cerebellar granule neurons. Interestingly, other E3 ligases have also been shown to act in an RING domain-independent manner. Sproul and colleagues reported that c-Cbl acts as an apoptotic brake in healthy neuronal cells (Sproul et al, 2009). Upon trophic deprivation, c$\mathrm{Cbl}$ protein levels rapidly decrease and neuronal apoptosis is induced by the sequential activation of MAP kinase cascades including the MLKs (mixed linage kinases), MKK4 and 7 (mitogen-activated protein kinase kinases) and finally JNKs (c-Jun N-terminal kinases), which leads to phosphorylation and activation of pro-apoptotic target proteins such as the transcription factor c-Jun. This effect was mimicked by RNAi-mediated knockdown of c-Cbl in PC12 cells upon NGF deprivation, while overexpression of c-Cbl and c-Cbl $\Delta$ RING protects neuronal cells against MLK overexpression-induced apoptosis. Furthermore, c-Cbl interacts with the JNK pathway components POSH and MLK3 in heterologous cells. Interestingly, the authors found that c-Cbl stabilizes MLK3 and suppresses its capacity to induce downstream apoptotic signaling. Thus, c-Cbl inhibits apoptotic signaling in healthy 
neurons by blocking MLK3 downstream signaling and JNK activation in an E3 ligase activity-independent manner (Sproul et al, 2009). In another report by Schwammborn and colleagues, Trim32, a homologue of the Drosophila brat protein, affects proliferation and differentiation of neuronal stem cells (NSCs) by two different signaling pathways. In dividing cortical progenitors, Trim32 is enriched in one of the two daughter cells, where it reduces the ability of NSCs to self-renew by ubiquitination and degradation of c-Myc, thereby providing a first step to induce neuronal differentiation (Kim et al, 2008). In addition, Trim32 can interact with RNase Argonaute 1 (Ago1) to activate the MicroRNA Let-7a that is known to regulate proliferation in normal and malignant cells, and is upregulated during neuronal differentiation (Johnson et al, 2007; Peng et al, 2008; Sempere et al, 2004; Yu et al, 2007). Hence, it is conceivable that several E3 ligases share a mechanistic concept, in which both ligase-dependent and independent functions co-exist.

\subsubsection{Wlds has no effect on the RNF157-knockdown induced dendritic phenotype}

The selective elimination of excessive neuronal projections can be found during developmental dendrite pruning as well as in response to injury-induced dendrite degeneration (Luo \& O'Leary, 2005; Tao \& Rolls, 2011; Williams \& Truman, 2005). Although these two processes involve similar morphological changes, Tao and colleagues reported that they are regulated by molecular distinct signaling pathways (Tao \& Rolls, 2011). Dendrite pruning in Drosophila ddaC neurons involves the action of apoptotic caspases, Katanin-p60L1 (Kat-60L1) (Lee et al, 2009b), IK2 (Lee et al, 2009b), Mical (Kirilly et al, 2009) and a $\mathrm{NAD}^{+}$signaling pathway to execute the degeneration process. In contrast, the process of injury-induced dendrite degeneration does neither require the apoptotic nor the pruning machinery. However, the components of the latter pathway require further research. Wlds was originally identified in the slow Wallerian degeneration mutant mouse where injury-induced axonal degeneration is significantly delayed as compared to wild type mice (Lunn et al, 1989). Recent studies have shown that Wlds is capable of blocking developmental dendrite pruning as well as injury-induced dendrite degeneration (Schoenmann et al, 2010; Tao \& Rolls, 2011). However, while the mechanism by which Wlds protects dendrites is unknown, it is assumed that Wlds controls NAD+ levels, which leads to a general inhibitory effect of both dendrite pruning and degeneration (Coleman \& Freeman, 2010; Tao \& Rolls, 2011). In the present study, I found that expression of Wlds in RNF157 knockdown neurons does not inhibit the RNF157 RNAi-induced reduction in total 
dendritic length. Hence, my results indicate that RNF157 regulates dendrite growth either downstream or independently of Wlds. In the experimental setup, cerebellar granule neurons were transfected at a time when they already possesses dendrites (DIV 4) and analyzed four days later. Interestingly, morphological analysis of control neurons between DIV 4-and 8 revealed that their total dendritic length remains relatively stable in this time window. Accordingly, these findings imply that cerebellar granule neurons do not undergo massive developmental pruning during the analyzed period. Hence, these findings suggest that RNF157 is not involved in dendrite pruning together with Wlds and support the role of RNF157 in dendrite maintenance.

\subsection{Fe65, a novel interactor of RNF157, acts in concert with RNF157 in the control of neuronal survival}

E3 ligases mediate the post-translational modification of proteins by covalently attaching one or several ubiquitin molecules. Substrate specificity is brought about by E3 ligases that mediate the transfer of ubiquitin from the E2 conjugating enzyme to the substrate. To identify substrates of RNF157, I carried out an interaction screen that led to the discovery of Fe65. Subsequently, this interaction was confirmed both in heterologous cells and in vivo. While RNF157 binds to the C-terminal part of Fe65 harboring the second PTB domain, Fe65 interacts with an N-terminal region of RNF157 (Figure 26).

Fe65 is a member of a multi-domain adaptor protein family, including Fe65L1 and Fe65L2, that is encoded in humans by separate genes on chromosome 11, 4 and 5, respectively (Blanco et al, 1998; Bressler et al, 1996; Tanahashi \& Tabira, 1999). Fe65 is a brain-enriched protein, whereby Fe65L1 and Fe65L2 are more widely expressed. While Fe65 null mice do not show any abnormalities, Fe65/Fe65L1 double knockout mice develop marginal zone heterotopias, middle crossing defects and axonal pathfinding abnormalities (Guenette et al, 2006) that resemble the phenotype seen in animals lacking all three amyloid precursor protein (APP) family members (Herms et al, 2004). These similarities were assumed to be caused by a decrease in APP/Fe65-dependent signaling, since Fe65 regulates APP trafficking and processing as well as the balance between amyloidogenic and non-amyloidogenic cleavage of APP (Ando et al, 2001; Borquez \& Gonzalez-Billault, 2012; Santiard-Baron et al, 2005). Similar to Fe65 and RNF157, the interaction between Fe65 and APP is mediated by the second PTB domain of Fe65 and a YENPTY reinternalisation signal in the cytoplasmic APP intracellular domain (AICD) (Fiore 
et al, 1995), thereby recruiting Fe65 to cell membranes (Minopoli et al, 2001). Hence, these data suggest that the binding of Fe65 to APP is an important regulatory mechanism to control APP processing and Aß production, which can be influenced by phosphorylation of APP at Thr668 or Tyr682 or by competing with Fe65 for binding to APP (Ando et al, 2001; Barbagallo et al, 2010; Kocherhans et al, 2010). In future studies, it will be of crucial importance to analyze if RNF157 regulates APP processing through interaction or modification of Fe65. Thus, I speculate that by binding of Fe65 to RNF157, less Fe65 will be available to associate with APP, which may affect APP processing and trafficking in neurons.

\subsubsection{Expression and localization of Fe65 during CNS development}

In my study, I analyzed the expression of Fe65 in different regions of the developing mouse brain. In the cerebellum, I found that Fe65 protein levels increase during development with the highest level of expression in adulthood. In contrast, while the expression of Fe65 was much weaker and mostly unchanged in the cortex, the expression of Fe65 in the hippocampus decreased from E18-P3 and was unchanged until adulthood. For the adult mouse brain, the expression levels of Fe65 are consistent with earlier in situ hybridization studies that demonstrate a high expression level of Fe65 mRNA in the hippocampus, cerebellum, olfactory bulb, cortex and habenular nucleus in the adult mouse brain (Bressler et al, 1996). Furthermore, Kesavapany and colleagues showed a widespread Fe65 immunoreactivity in the adult mouse brain with high immunoreactivity in cerebellum and hippocampus and a decreased staining pattern in the neocortex (Kesavapany et al, 2002). At the subcellular level, I found Fe65 immunoreactivity in the nucleus, the soma and in the processes of neurons. Although Fe65 has been identified as a strictly cytoplasmic protein (Delatour et al, 2001; Kesavapany et al, 2002), increasing evidence demonstrates a nuclear localization of endogenous Fe65 in neurons (Barbato et al, 2005; Lau et al, 2008; Minopoli et al, 2001). My results are consistent with the findings by Barbato and colleagues, who showed a somatoneuritic as well as nuclear localization of Fe65 in cerebellar granule and hippocampal neurons (Barbato et al, 2005).

Interestingly, the expression pattern of RNF157 is similar to that of Fe65 in the cerebellum. However, while RNF157 levels decrease in adulthood in the cortex, the expression of Fe65 is unchanged throughout development. Conversely, while Fe65 expression decreases during development in the hippocampus, the expression of RNF157 
is unchanged. In addition, RNF157 and Fe65 display an overlapping subcellular staining pattern in neurons. Hence, my results indicate that RNF157 and Fe65 have an important function together during mammalian brain development.

\subsubsection{Fe65 is ubiquitinated by the E3 ligase RNF157}

Since Fe65 was identified as a novel interactor of RNF157, I subsequently analyzed the ubiquitination of Fe65 in neurons. My results indicate that Fe65 is degraded in a proteasome-dependent manner, since Fe65 levels increase upon selective inhibition of the proteasome in cerebellar granule neurons. Interestingly, Fe65 isolated from cultured cerebellar granule neurons harbors Lys48-and Lys63-linked ubiquitin modifications, suggesting that Fe65 is both marked for proteasomal degradation as well as in a nondegradative manner, which can lead to changes in protein localization, activity or interaction with binding partners (for review see: (Kawabe \& Brose, 2011; Schnell \& Hicke, 2003). Having confirmed RNF157's E3 ligase activity, in vitro ubiquitination assays suggest that RNF157 acts in a complex with the E2 UbcH5a to either multimonoubiquitinate or diubiquitinate Fe65 in vitro. Strikingly, no changes in Fe65 protein levels were observed in cortical lysates of RNF157 heterozygous mice in comparison to control littermates (courtesy of S.J Lee). Hence, I conclude that RNF157 regulates the function, interaction or localization of Fe65 by a non-degradative ubiquitin modification. Accordingly, RNF157 does not target Fe65 for proteasomal degradation. Interestingly, Lee and colleagues previously identified neuronal precursor cell expressed developmentally down regulated (NEDD) 4-2 as an E3 ligase for Fe65 and suggest that NEDD4-2 targets Fe65 for proteasomal degradation (Lee et al, 2009a). This is accompanied by the Lys48-linked polyubiquitination of Fe65 and would explain the accumulation of Fe65 seen upon inhibition of the proteasome. Since proteins can be targeted by independent E3 ligases (Amati, 2004; Nishitani et al, 2006), it is conceivable that Fe65 is targeted for proteasomal degradation by NEDD4-2, whereas ubiquitination of Fe65 by RNF157 leads to a regulatory modification of Fe65 in neurons.

Proteasome-independent ubiquitin modifications have been further shown for other E3 ligases (Komander \& Rape, 2012; Schnell \& Hicke, 2003). Among those, the E3 ligase neuronal precursor cell expressed and developmentally downregulated protein (Nedd) 4-1 regulates dendrite outgrowth and complexity in a trimeric complex with Rap2a and TNIK by mono-or diubiquitination of Rap2a, thereby blocking the activity of Rap2a, which in turn 
leads to reduced activity of downstream effector kinases of the TNIK family followed by dendrite outgrowth. Thus, ubiquitination of Rap2a by Nedd4-1 regulates the activity of Rap2a (Kawabe et al, 2010). In addition, monoubiquitination of p53 by the E3 ligase Mdm2 induces its export from the nucleus, which is assumed to be mediated by changes in the accessibility of the nuclear export signal of p53 to the export machinery (Li et al, 2003). Thus, it is conceivable that the RNF157-mediated mono-or diubiquitination of Fe65 may modulate the subcellular localization of Fe65 in neurons or the ability of Fe65 to bind to its interaction partners APP, LRP or Tip60 (reviewed in: (Borquez \& Gonzalez-Billault, 2012)). Interestingly, the E3 ligase Mgrn1 was shown to multimonoubiquitinate TSG101, a key component of the ESCRT-I complex (Kim et al, 2007a), which suggests that monoubiquitination might be a general feature of the E3 ligases RNF157 and Mgrn1.

In future experiments, it will be important the bolster the finding that Fe65 is a substrate of RNF157 by demonstrating that the RING domain is instrumental to Fe65 ubiquitination and by identifying the target lysine(s) of Fe65, which act as ubiquitin acceptors. Finally, it will be important to determine if RNF157 mediates mono-or diubiquitination of Fe65 using a polymerization-defective ubiquitin mutant.

\subsubsection{RNF157 and Fe65 act in a linear pathway to control neuronal survival}

The E3 ligase RNF157 was uncovered as a novel regulator of dendrite morphogenesis and neuronal survival in vitro. In addition, I identified the brain-enriched adaptor protein Fe65 as a novel interactor of RNF157. In vitro ubiquitination assays indicate that RNF157 ubiquitinates Fe65 in a non-proteolytic manner. Consistent with these results, epistasis analysis of RNF157 and Fe65 in neurons suggest that Fe65 acts downstream of RNF157 in the control of neuronal survival. While overexpression of Fe65 triggers cell death, RNAi-mediated knockdown of Fe65 significantly reduces starvationinduced apoptosis in cerebellar granule neurons. My findings further demonstrate that Fe65 operates in the nucleus to initiate neuronal apoptosis. These results were accompanied by structure-function analysis, which show that nuclear Fe65 dramatically increases cell death in cerebellar granule neurons. In summary, my findings suggest that RNF157 and Fe65 are components of a cell-intrinsic signaling pathway that controls neuronal survival.

In future studies, it will be important to gain further insight into the RNF157-Fe65 underlying mechanism regulating neuronal survival. Interestingly, Fe65 was shown to 
activate together with APP the transcriptional activity of the histone acetyltransferase Tip60 (Cao \& Sudhof, 2001). Fe65 is assumed to adopt a closed conformation in the cytoplasm, which can be reversed by binding to the cytoplasmatic tail of APP (AICD), followed by the recruitment and phosphorylation of Tip60 by cyclin-dependent kinases (CDKs) (Cao \& Sudhof, 2004; Hass \& Yankner, 2005; Lemercier et al, 2003). The release of the complex from the plasma membrane in response to DNA damage, cellular stress or other unknown stimuli can be induced via two complementary mechanisms: either by subsequent cleavage of APP by $ß$-and $\gamma$-secretase or by the phosphorylation of APP at Thr668 or Tyr682, which attenuates the binding affinity between Fe65 and APP (Ando et al, 2001; Barbagallo et al, 2010; Cao \& Sudhof, 2001; Nakaya \& Suzuki, 2006; Radzimanowski et al, 2008). The complex then translocates to the nucleus to regulate gene expression, repair of DNA double strand-breaks (DSB) and apoptosis (Baek et al, 2002; Gaughan et al, 2001; Ikura et al, 2000; Kimberly et al, 2001; Kinoshita et al, 2002a; Kinoshita et al, 2002b; Passer et al, 2000). In addition, the induction of apoptosis by the AICD/Fe65/Tip60 signaling pathway involves the upregulation of pro-apoptotic proteins including p53 (Alves da Costa et al, 2006; Kim et al, 2007b; Kinoshita et al, 2002a). Interestingly, Kim and colleagues reported that the intracellular domain of Notch 1 (Notch1-IC) suppresses AICD-induced cell death of neuroblastoma cells by physical disruption of the AICD/Fe65/Tip60 complex (Kim et al, 2007b). In view of these observations, I hypothesize that the RNF157-mediated ubiquitination of Fe65 may lead to the following consequences: (I) Interaction of RNF157 with Fe65 competes for the binding of Fe65 to APP, thereby diminishing phosphorylation and activation of Tip60. (II) While the C-terminal Fe65 fragment (Fe65aa525-711) translocates to the nucleus and induces apoptosis, Fe65aa326-711 is equally distributed within the cell and only shows a moderate induction of apoptosis. In addition, Fe65 is ubiquitinated by RNF157 in a non-degradative manner. Hence, I postulate that ubiquitination of Fe65 by RNF157 regulates the localization of Fe65. Accordingly, ubiquitination of Fe65 might inhibit nuclear translocation of Fe65, which inhibits the activation of transcription mediated by Tip60, thereby preventing the induction of apoptosis (Figure 42). This is supported by the work from Cao and Südhof, who showed that cytoplasmic NES-Fe65 together with APP was unable to activate transcription in a Gal4-Tip60 transactivation assay. In contrast, translocation of AICD in this context was apparently unnecessary for activation of transcription (Cao \& Sudhof, 2004). 
To confirm this putative signaling pathway, it will be essential to elucidate if the RNF157-Fe65 pathway regulates neuronal survival together with APP or Tip60. Furthermore, it will be important to determine if ubiquitination of Fe65 by RNF157 inhibits its nuclear translocation, thereby suppressing the transcription of pro-apoptotic target genes. In this context, it will be crucial to analyze the localization of Fe65 in neurons of RNF157 wild type and heterozygous animals to verify that ubiquitination of Fe65 by RNF157 affects its localization in neurons.

\subsubsection{RNF157 and death receptor 6 (DR6) act in a linear pathway to control neuronal survival}

DR6 is a member of the tumor necrosis factor (TNF) receptor superfamily, which harbors a cysteine-rich, extracellular domain and a conserved intracellular death domain (DD). Expression of DR6 in neurons induces cell death and axon degeneration (Nikolaev et al, 2009). However, the precise mechanism of DR6-induced cell death still remains unknown. Interestingly, Nikolaev and colleagues identified the amino-terminal fragment of APP (N-APP) as a novel ligand of DR6 that is produced upon trophic deprivation of sympathetic neurons in a BACE-dependent manner. Binding of N-APP to DR6 subsequently triggers axonal pruning and neuronal apoptosis. Interestingly, Fe65 is known to regulate APP processing as well as the balance between amyloidogenic and non-amyloidogenic cleavage (Ando et al, 2001; Borquez \& Gonzalez-Billault, 2012; Santiard-Baron et al, 2005). Thus, since Fe65 was identified as an interactor of RNF157, I analyzed if the increase of apoptosis upon RNF157 RNAi can be inhibited by simultaneous knockdown of DR6. Here, I found that RNF157 and DR6 act in a linear pathway to control neuronal survival. Hence, since RNF157 and APP may compete for the binding of Fe65, I hypothesize that RNF157 loss-of-function increases the binding of Fe65 to APP, which may upregulate the processing of APP that leads to elevated levels of N-APP followed by the induction of apoptosis through binding of N-APP to DR6. However, to reveal this possibility, it will be important to establish a biochemical and functional relationship of RNF157, Fe65 and APP together with DR6 in neuronal survival. 


\subsection{Generation and characterization of a mouse model lacking RNF157 function}

The UPS has been implicated in a plethora of different signaling events in the mammalian brain (Kawabe \& Brose, 2011; Tuoc \& Stoykova, 2010; Yi \& Ehlers, 2007). In particular, there is increasing evidence that E3 ligases control important aspects of neuronal development including neurogenesis, migration, neuritogenesis and synapse formation (Kawabe \& Brose, 2011). In this study, the E3 ligase RNF157 has been identified as an important regulator of dendrite morphogenesis and neuronal survival in postmitotic neurons in vitro. Complementary, an RNF157 gene trap mouse model was generated in this work to elucidate the in vivo physiological function of RNF157 in mammalian brain development.

Analysis of RNF157 heterozygous mice showed a reduction of RNF157 protein in the brain of adult mice. Interestingly, RNF157 heterozygous animals seemed to be physically healthy and showed no changes in the gross morphology of the brain in comparison to wild type littermates. Subsequently, PCR-genotyped RNF157 heterozygous mice were crossed to obtain homozygous mice. Among the genotyped postnatal littermates (P5 and 3 weeks), none were homozygous for the trapped allele. Subsequently, RNF157 embryos at E10 and E7 were analyzed. However, so far none were homozygous for the trapped allele. Hence, these results suggest that disruption of RNF157 function causes early embryonic lethality of RNF157 homozygous embryos (Table 2) and that RNF157 is essential during mammalian embryonic development. Deletion of Mgrn1 exhibits a similar effect on embryonic viability, which is the result of congenital heart defects and abnormal patterning of the Right-Left axis (He et al, 2003; Phillips, 1963). The surviving Mgrn1 null mice display other phenotypes including dark pigmentation and adult-onset of progressive spongiform neurodegeneration ( $\mathrm{He}$ et al, 2003). Hence, these findings demonstrate the crucial importance of RNF157 and Mgrn1 during embryonic development

In future studies, it will be important to define at which stage deletion of RNF157 disrupts embryonic development. Thus, further expression analyses of RNF157 during embryonic development are required. Since the RNF157 heterozygous mice survive until adulthood, it will be important to analyze if they develop age-dependent neurodegeneration as reported for Mgrn1 homozygous mice. Moreover, behavioral tests of RNF157 heterozygous mice grant further insight into the function of RNF157 in the brain. 


\subsection{Conclusion}

My work has uncovered that the brain-dominant RING E3 ligase RNF157 is an important regulator of dendrite morphogenesis and neuronal survival. In addition, the adaptor protein Fe65 has been identified as a novel key target of RNF157, which is ubiquitinated in a non-degradative manner. Functional analyses revealed that Fe65 is a pro-apoptotic protein, which operates in the nucleus. Consistent with these results, epistasis analysis revealed that Fe65 acts downstream of RNF157 in the control of neuronal survival. Hence, my research has uncovered an RNF157-Fe65 pathway that regulates neuronal survival (Figure 42). The work was accompanied by the generation of an RNF157 mouse model, which demonstrates the importance of RNF157 during embryonic development. Taken together, my study significantly improves our understanding of UPS-regulated mechanisms, which are involved in neuronal survival. Impairment of the highly complex and orchestrated pathways that regulate ubiquitination is often sufficient to cause a widespread spectrum of neurological disorders. Hence, my research may provide a novel link between perturbations in ubiquitination and neurodegenerative diseases.
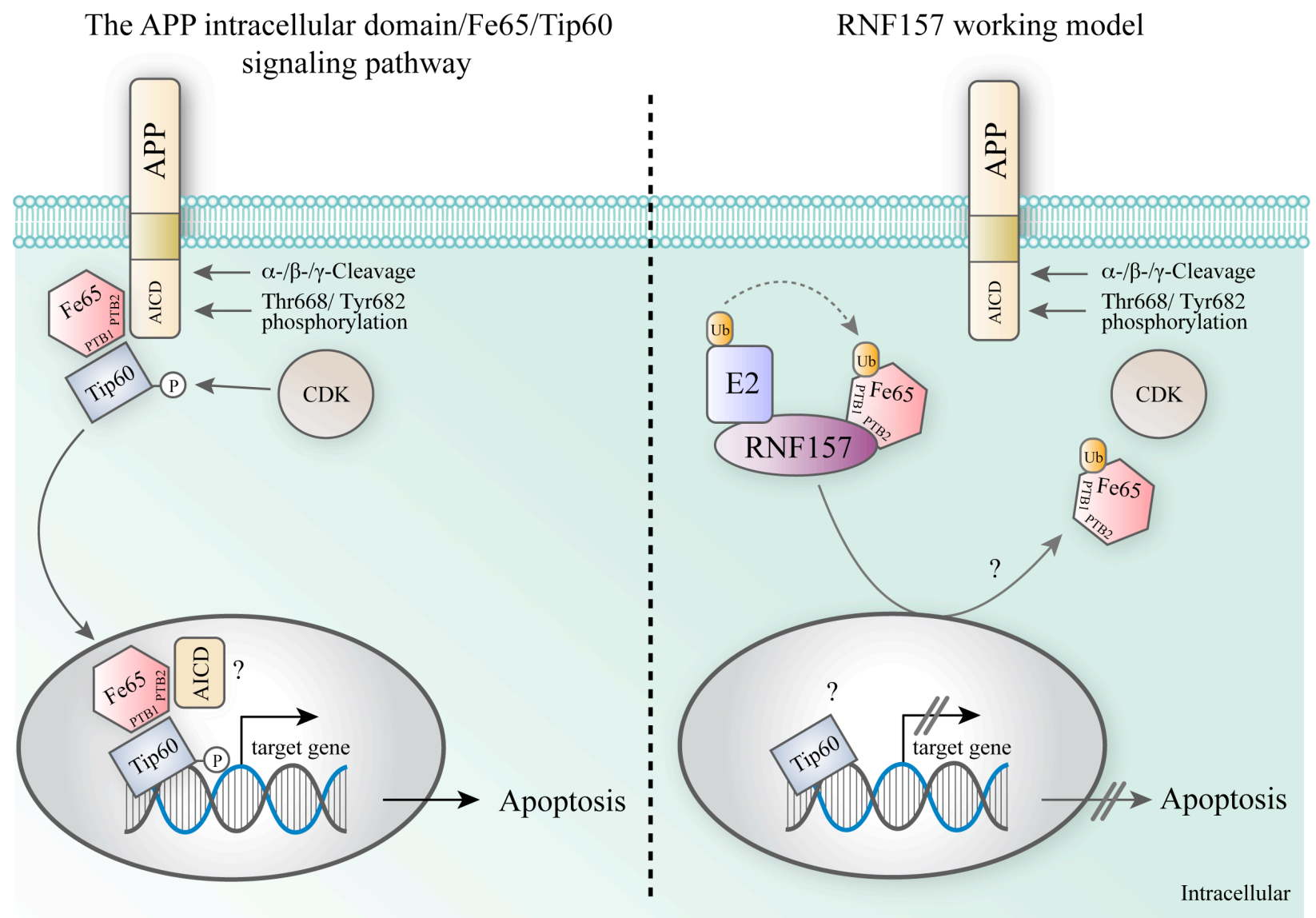
Figure 42. A working model for the E3 ligase RNF157 and its novel interactor Fe65 in neuronal survival. The interaction of Fe65 and APP leads to the recruitment and activation of the histone acetyltransferase Tip60 by CDK-dependent phosphorylation (Hass \& Yankner, 2005). Then, this multimeric complex is released into the cytoplasm either by proteolytic processing of APP by $\alpha$-or $\beta$-secretase followed by $\gamma$-secretase or by phosphorylation of APP at Thr668 or Tyr682, which reduces the binding affinity of Fe65 and APP (Ando et al, 2001; Barbagallo et al, 2010; Cao \& Sudhof, 2001; Nakaya \& Suzuki, 2006; Radzimanowski et al, 2008). This enables nuclear translocation of the Fe65-dependent signaling complex to induce the transcription of target genes and apoptosis. In the putative RNF157 working model however, interaction of RNF157 to the C-terminus of Fe65 reduces the binding of Fe65 to APP, which suppresses recruitment and activation of Tip60. Furthermore, RNF157-dependent ubiquitination of Fe65 inhibits the translocation of Fe65 to the nucleus, thereby attenuating neuronal apoptosis. CDK, cyclin-dependent kinase; AICD, APP intracellular domain; P, phosphate group; Ub, ubiquitin; PTB, phosphotyrosine binding domain. 


\section{Appendix}

\subsection{Alignment of mouse, human and predicted rat cDNA sequence}

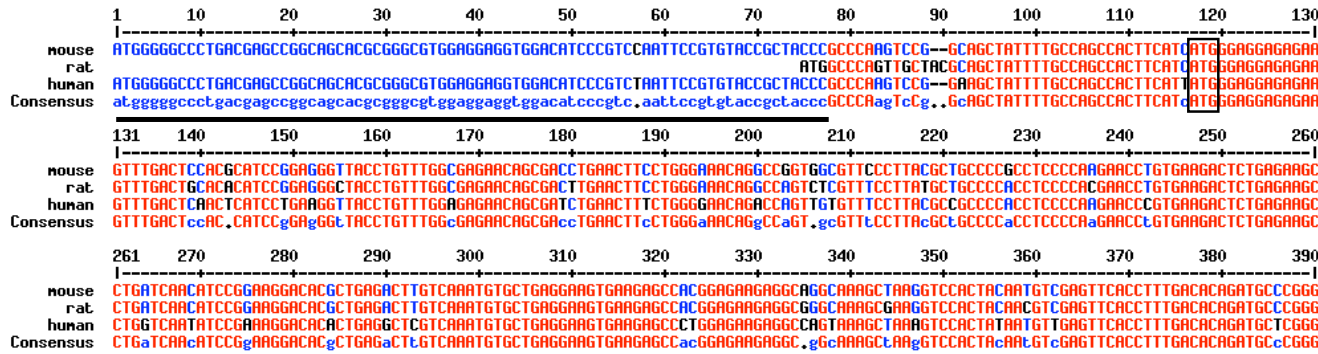
$391 \quad 400$
$410 \quad 420$
430
$440 \quad 450$
$460 \quad 470$
480
$500 \quad 510 \quad 520$

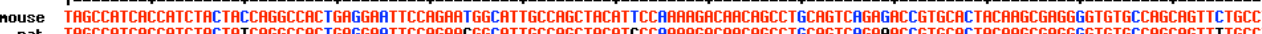

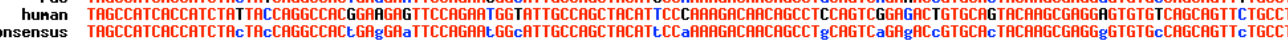

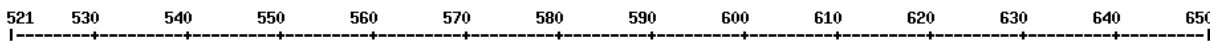

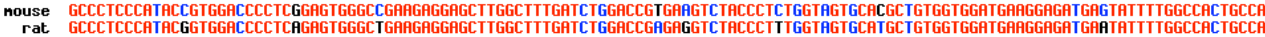

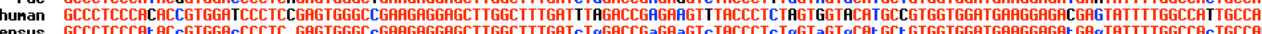

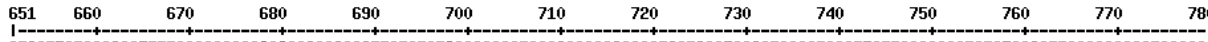

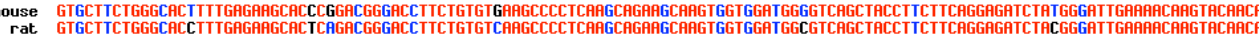

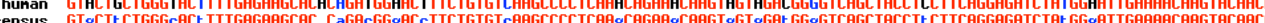

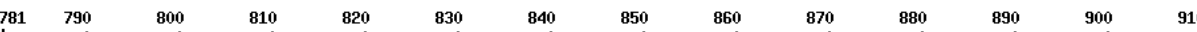

nouse CACAAGATTCCARGGTGGCAGRGGACGACGTCAGTGACAACAGCGCTGAGGGTGTGGTGTGTCTCTCTGACGTCCGCGATACTITGATCCTGCCCTGCCGGCACCTCTGCCTCTGCAACACCTGCGCAG
rat

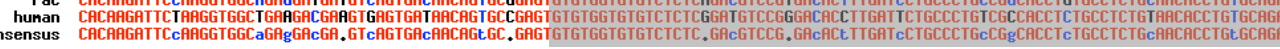

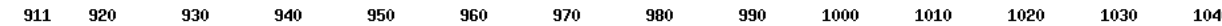

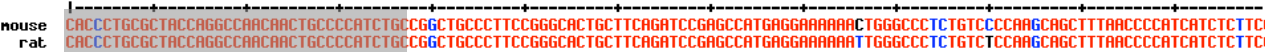

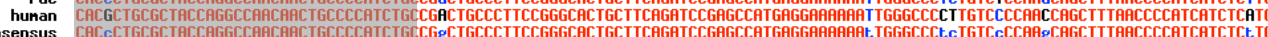

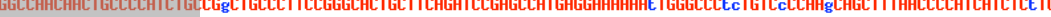

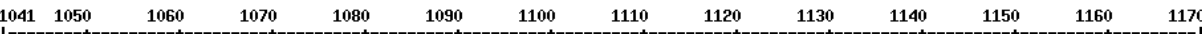

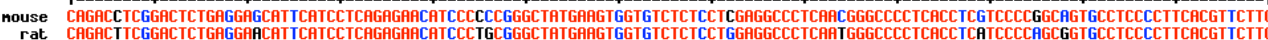

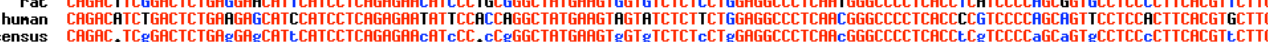

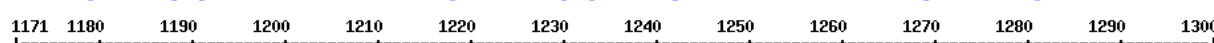

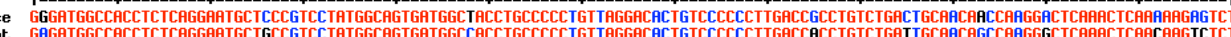

rat GAGATGGCCACCTCTCAGGAATGCCTGCCGTCCTATGGCAGTGRTGGCCACCTGCCCCCTGTTAGGACACTGTCCCCCCTTGACCACC TGTCTGATTGCAACAGCCARGGGCTCARACTCAACAAGTCTC

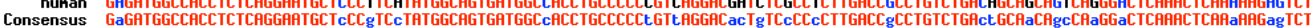

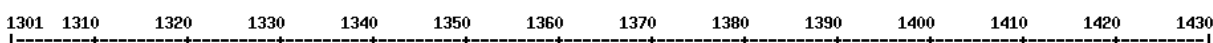

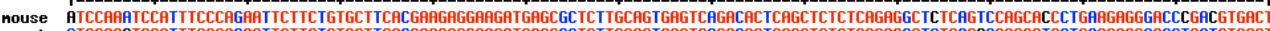

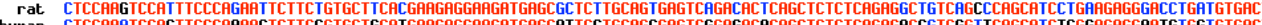

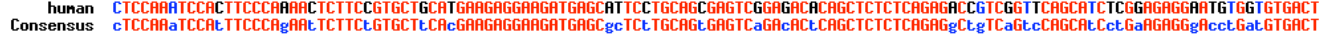

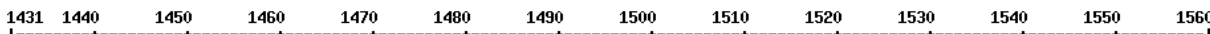

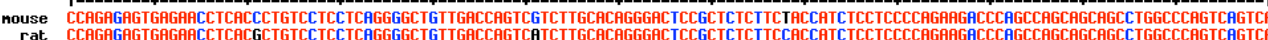

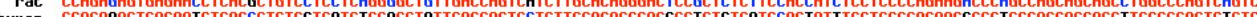

Consensus CCAGAgAGTGAGAfACCTCACcCTGTCcTCcTCaGGgGCTgTTGACCAGTCgTCTTGCACAGGGACECCgCTcTCETCCACcATcTCCTCCCCAGAAGaCCCaGCCAGCAGCAGCCTGGCCCAGTCaGTCH

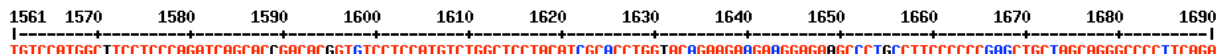

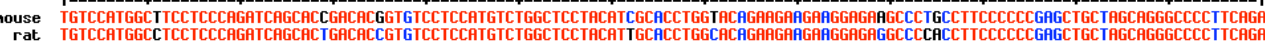

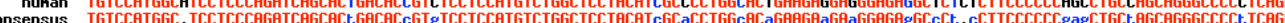

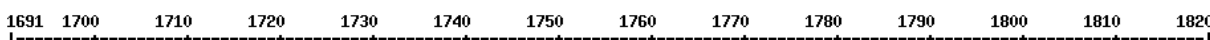

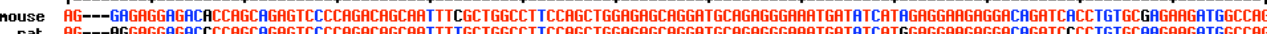

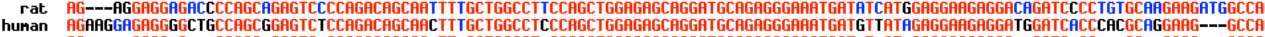

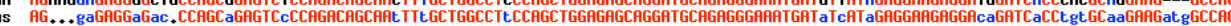

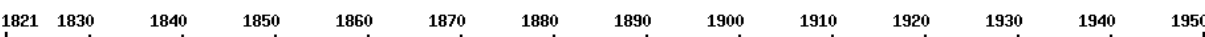

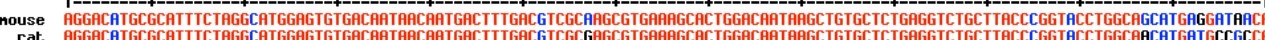

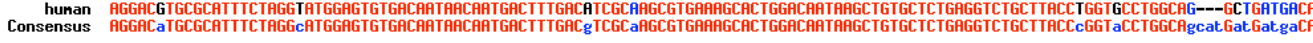

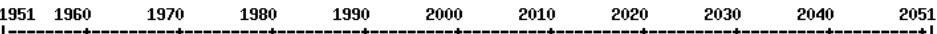

nouse CTGTCAACTGTCGCCATACCCAGCGCCGGCGCTTATCCTCCAGCAGCCTGGAGGACCCTGRGGAGGACAGGCCTIGTGTATGGGATCCTATGGCTGTCTG

hunan ATGCGGTCAGTCGGAATGCCCAGCGGCGGGCCCTTGTCATCCAGCAGCCTGGGGGACTCTGAGAGG---AGGCCCTGTGTGGGGGGGCCCTTGGGCTGTCTGA 
Symbols and colors. High-consensus color=red; Low consensus color=blue; Neutral color=black. A highly conserved residue appears in high-consensus color and as an uppercase letter in the consensus line. A weakly conserved residue appears in lowconsensus color and as a lowercase letter in the consensus line. Other residues appear in neutral color. A position with no conserved residue is represented by a dot in the consensus line. The second conserved in-frame start codon is highlighted by a black square. Black line indicates the different N-terminal sequences of mouse, human and rat RNF157. The RING domain is shaded in grey. 


\section{References}

Adams NC, Tomoda T, Cooper M, Dietz G, Hatten ME (2002) Mice that lack astrotactin have slowed neuronal migration. Development 129: 965-972

Aizawa H, Hu SC, Bobb K, Balakrishnan K, Ince G, Gurevich I, Cowan M, Ghosh A (2004) Dendrite development regulated by CREST, a calcium-regulated transcriptional activator. Science 303: 197-202

Ali SF, Binienda ZK, Imam SZ (2011) Molecular aspects of dopaminergic neurodegeneration: gene-environment interaction in parkin dysfunction. Int J Environ Res Public Health 8: 4702-4713

Almeida A, Bolanos JP, Moreno S (2005) Cdh1/Hct1-APC is essential for the survival of postmitotic neurons. J Neurosci 25: 8115-8121

Alves da Costa C, Sunyach C, Pardossi-Piquard R, Sevalle J, Vincent B, Boyer N, Kawarai T, Girardot N, St George-Hyslop P, Checler F (2006) Presenilin-dependent gamma-secretasemediated control of p53-associated cell death in Alzheimer's disease. J Neurosci 26: 63776385

Amati B (2004) Myc degradation: dancing with ubiquitin ligases. Proc Natl Acad Sci U S A 101: 8843-8844

Ando K, Iijima KI, Elliott JI, Kirino Y, Suzuki T (2001) Phosphorylation-dependent regulation of the interaction of amyloid precursor protein with Fe65 affects the production of beta-amyloid. J Biol Chem 276: 40353-40361

Anton ES, Marchionni MA, Lee KF, Rakic P (1997) Role of GGF/neuregulin signaling in interactions between migrating neurons and radial glia in the developing cerebral cortex. Development 124: 3501-3510

Aravind L, Iyer LM, Koonin EV (2003) Scores of RINGS but no PHDs in ubiquitin signaling. Cell Cycle 2: 123-126

Aravind L, Koonin EV (2000) The U box is a modified RING finger - a common domain in ubiquitination. Curr Biol 10: R132-134

Arnoult D, Gaume B, Karbowski M, Sharpe JC, Cecconi F, Youle RJ (2003) Mitochondrial release of AIF and EndoG requires caspase activation downstream of Bax/Bak-mediated permeabilization. EMBO J 22: 4385-4399

Ayala R, Shu T, Tsai LH (2007) Trekking across the brain: the journey of neuronal migration. Cell 128: 29-43 
Baek SH, Ohgi KA, Rose DW, Koo EH, Glass CK, Rosenfeld MG (2002) Exchange of N-CoR corepressor and Tip60 coactivator complexes links gene expression by NF-kappaB and beta-amyloid precursor protein. Cell 110: 55-67

Bagher P, Jiao J, Owen Smith C, Cota CD, Gunn TM (2006) Characterization of Mahogunin Ring Finger-1 expression in mice. Pigment Cell Res 19: 635-643

Baker RE, Dijkhuizen PA, Van Pelt J, Verhaagen J (1998) Growth of pyramidal, but not nonpyramidal, dendrites in long-term organotypic explants of neonatal rat neocortex chronically exposed to neurotrophin-3. Eur J Neurosci 10: 1037-1044

Barbagallo AP, Weldon R, Tamayev R, Zhou D, Giliberto L, Foreman O, D'Adamio L (2010) $\operatorname{Tyr}(682)$ in the intracellular domain of APP regulates amyloidogenic APP processing in vivo. PLoS One 5: e15503

Barbato C, Canu N, Zambrano N, Serafino A, Minopoli G, Ciotti MT, Amadoro G, Russo T, Calissano P (2005) Interaction of Tau with Fe65 links tau to APP. Neurobiol Dis 18: 399408

Barnes AP, Polleux F (2009) Establishment of axon-dendrite polarity in developing neurons. Annu Rev Neurosci 32: 347-381

Becker EB, Bonni A (2004) Cell cycle regulation of neuronal apoptosis in development and disease. Prog Neurobiol 72: 1-25

Benn SC, Woolf CJ (2004) Adult neuron survival strategies--slamming on the brakes. Nat Rev Neurosci 5: 686-700

Bennett EJ, Shaler TA, Woodman B, Ryu KY, Zaitseva TS, Becker CH, Bates GP, Schulman H, Kopito RR (2007) Global changes to the ubiquitin system in Huntington's disease. Nature 448: 704-708

Berglund EO, Murai KK, Fredette B, Sekerkova G, Marturano B, Weber L, Mugnaini E, Ranscht B (1999) Ataxia and abnormal cerebellar microorganization in mice with ablated contactin gene expression. Neuron 24: 739-750

Besson A, Dowdy SF, Roberts JM (2008) CDK inhibitors: cell cycle regulators and beyond. Dev Cell 14: 159-169

Bhardwaj A, Aggarwal BB (2003) Receptor-mediated choreography of life and death. J Clin Immunol 23: 317-332

Blanco G, Irving NG, Brown SD, Miller CC, McLoughlin DM (1998) Mapping of the human and murine X11-like genes (APBA2 and apba2), the murine Fe65 gene (Apbb1), and the human Fe65-like gene (APBB2): genes encoding phosphotyrosine-binding domain proteins that interact with the Alzheimer's disease amyloid precursor protein. Mamm Genome 9: 473-475 
Borg JP, Ooi J, Levy E, Margolis B (1996) The phosphotyrosine interaction domains of X11 and FE65 bind to distinct sites on the YENPTY motif of amyloid precursor protein. Mol Cell Biol 16: 6229-6241

Borquez DA, Gonzalez-Billault C (2012) The amyloid precursor protein intracellular domain-fe65 multiprotein complexes: a challenge to the amyloid hypothesis for Alzheimer's disease? Int J Alzheimers Dis 2012: 353145

Bouwmeester T, Kim S, Sasai Y, Lu B, De Robertis EM (1996) Cerberus is a head-inducing secreted factor expressed in the anterior endoderm of Spemann's organizer. Nature 382: 595-601

Bradke F, Dotti CG (1999) The role of local actin instability in axon formation. Science 283: 1931-1934

Bressler SL, Gray MD, Sopher BL, Hu Q, Hearn MG, Pham DG, Dinulos MB, Fukuchi K, Sisodia SS, Miller MA, Disteche CM, Martin GM (1996) cDNA cloning and chromosome mapping of the human Fe65 gene: interaction of the conserved cytoplasmic domains of the human beta-amyloid precursor protein and its homologues with the mouse Fe65 protein. Hum Mol Genet 5: 1589-1598

Broadbelt K, Byne W, Jones LB (2002) Evidence for a decrease in basilar dendrites of pyramidal cells in schizophrenic medial prefrontal cortex. Schizophr Res 58: 75-81

Brose K, Tessier-Lavigne M (2000) Slit proteins: key regulators of axon guidance, axonal branching, and cell migration. Curr Opin Neurobiol 10: 95-102

Butler SJ, Dodd J (2003) A role for BMP heterodimers in roof plate-mediated repulsion of commissural axons. Neuron 38: 389-401

Cao X, Sudhof TC (2001) A transcriptionally [correction of transcriptively] active complex of APP with Fe65 and histone acetyltransferase Tip60. Science 293: 115-120

Cao X, Sudhof TC (2004) Dissection of amyloid-beta precursor protein-dependent transcriptional transactivation. J Biol Chem 279: 24601-24611

Carri MT, Ferri A, Cozzolino M, Calabrese L, Rotilio G (2003) Neurodegeneration in amyotrophic lateral sclerosis: the role of oxidative stress and altered homeostasis of metals. Brain Res Bull 61: 365-374

Chang C, Hemmati-Brivanlou A (1998) Cell fate determination in embryonic ectoderm. $J$ Neurobiol 36: 128-151

Charron F, Stein E, Jeong J, McMahon AP, Tessier-Lavigne M (2003) The morphogen sonic hedgehog is an axonal chemoattractant that collaborates with netrin-1 in midline axon guidance. Cell 113: 11-23 
Charych EI, Akum BF, Goldberg JS, Jornsten RJ, Rongo C, Zheng JQ, Firestein BL (2006) Activity-independent regulation of dendrite patterning by postsynaptic density protein PSD-95. J Neurosci 26: 10164-10176

Chen JL, Nedivi E (2010) Neuronal structural remodeling: is it all about access? Curr Opin Neurobiol 20: 557-562

Chu J, Hong NA, Masuda CA, Jenkins BV, Nelms KA, Goodnow CC, Glynne RJ, Wu H, Masliah E, Joazeiro CA, Kay SA (2009) A mouse forward genetics screen identifies LISTERIN as an E3 ubiquitin ligase involved in neurodegeneration. Proc Natl Acad Sci U S A 106: 20972103

Cline HT (2001) Dendritic arbor development and synaptogenesis. Curr Opin Neurobiol 11: 118-126

Coleman MP, Conforti L, Buckmaster EA, Tarlton A, Ewing RM, Brown MC, Lyon MF, Perry VH (1998) An 85-kb tandem triplication in the slow Wallerian degeneration (Wlds) mouse. Proc Natl Acad Sci U S A 95: 9985-9990

Coleman MP, Freeman MR (2010) Wallerian degeneration, wld(s), and nmnat. Annu Rev Neurosci 33: 245-267

Compton MM (1992) A biochemical hallmark of apoptosis: internucleosomal degradation of the genome. Cancer Metastasis Rev 11: 105-119

Conforti L, Tarlton A, Mack TG, Mi W, Buckmaster EA, Wagner D, Perry VH, Coleman MP (2000) A Ufd2/D4Cole1e chimeric protein and overexpression of Rbp7 in the slow Wallerian degeneration (WldS) mouse. Proc Natl Acad Sci U S A 97: 11377-11382

Cota CD, Bagher P, Pelc P, Smith C0, Bodner CR, Gunn TM (2006) Mice with mutations in Mahogunin ring finger-1 (Mgrn1) exhibit abnormal patterning of the left-right axis. Dev Dyn 235: 3438-3447

Cowan WM, Fawcett JW, O'Leary DD, Stanfield BB (1984) Regressive events in neurogenesis. Science 225: 1258-1265

Coyle JT, Puttfarcken P (1993) Oxidative stress, glutamate, and neurodegenerative disorders. Science 262: 689-695

Craig AM, Banker G (1994) Neuronal polarity. Annu Rev Neurosci 17: 267-310

Cryns V, Yuan J (1998) Proteases to die for. Genes Dev 12: 1551-1570

Daugas E, Susin SA, Zamzami N, Ferri KF, Irinopoulou T, Larochette N, Prevost MC, Leber B, Andrews D, Penninger J, Kroemer G (2000) Mitochondrio-nuclear translocation of AIF in apoptosis and necrosis. FASEB J 14: 729-739

De Robertis EM, Sasai Y (1996) A common plan for dorsoventral patterning in Bilateria. Nature 380: 37-40 
Degterev A, Boyce M, Yuan J (2003) A decade of caspases. Oncogene 22: 8543-8567

Delatour B, Mercken L, El Hachimi KH, Colle MA, Pradier L, Duyckaerts C (2001) FE65 in Alzheimer's disease: neuronal distribution and association with neurofibrillary tangles. Am J Pathol 158: 1585-1591

Demyanenko GP, Tsai AY, Maness PF (1999) Abnormalities in neuronal process extension, hippocampal development, and the ventricular system of L1 knockout mice. J Neurosci 19: 4907-4920

Deshaies RJ, Joazeiro CA (2009) RING domain E3 ubiquitin ligases. Annu Rev Biochem 78: $399-434$

Deshmukh M, Johnson EM, Jr. (1998) Evidence of a novel event during neuronal death: development of competence-to-die in response to cytoplasmic cytochrome c. Neuron 21: 695-705

Dessaud E, McMahon AP, Briscoe J (2008) Pattern formation in the vertebrate neural tube: a sonic hedgehog morphogen-regulated transcriptional network. Development 135: 24892503

Dotti CG, Sullivan CA, Banker GA (1988) The establishment of polarity by hippocampal neurons in culture. J Neurosci 8: 1454-1468

Duilio A, Faraonio R, Minopoli G, Zambrano N, Russo T (1998) Fe65L2: a new member of the Fe65 protein family interacting with the intracellular domain of the Alzheimer's betaamyloid precursor protein. Biochem J 330 ( Pt 1): 513-519

Duilio A, Zambrano N, Mogavero AR, Ammendola R, Cimino F, Russo T (1991) A rat brain mRNA encoding a transcriptional activator homologous to the DNA binding domain of retroviral integrases. Nucleic Acids Res 19: 5269-5274

Edmondson JC, Liem RK, Kuster JE, Hatten ME (1988) Astrotactin: a novel neuronal cell surface antigen that mediates neuron-astroglial interactions in cerebellar microcultures. $J$ Cell Biol 106: 505-517

Emoto K (2012) Signaling mechanisms that coordinate the development and maintenance of dendritic fields. Curr Opin Neurobiol

Englund C, Fink A, Lau C, Pham D, Daza RA, Bulfone A, Kowalczyk T, Hevner RF (2005) Pax6, Tbr2, and Tbr1 are expressed sequentially by radial glia, intermediate progenitor cells, and postmitotic neurons in developing neocortex. J Neurosci 25: 247-251

Ermekova KS, Zambrano N, Linn H, Minopoli G, Gertler F, Russo T, Sudol M (1997) The WW domain of neural protein FE65 interacts with proline-rich motifs in Mena, the mammalian homolog of Drosophila enabled. J Biol Chem 272: 32869-32877

Esposti MD (2002) The roles of Bid. Apoptosis 7: 433-440 
Falk S, Sommer L (2009) Stage- and area-specific control of stem cells in the developing nervous system. Curr Opin Genet Dev 19: 454-460

Famulski JK, Trivedi N, Howell D, Yang Y, Tong Y, Gilbertson R, Solecki DJ (2010) Siah regulation of Pard3A controls neuronal cell adhesion during germinal zone exit. Science 330: $1834-1838$

Farber E (1994) Programmed cell death: necrosis versus apoptosis. Mod Pathol 7: 605-609

Fiore F, Zambrano N, Minopoli G, Donini V, Duilio A, Russo T (1995) The regions of the Fe65 protein homologous to the phosphotyrosine interaction/phosphotyrosine binding domain of Shc bind the intracellular domain of the Alzheimer's amyloid precursor protein. $J$ Biol Chem 270: 30853-30856

Fishell G, Hatten ME (1991) Astrotactin provides a receptor system for CNS neuronal migration. Development 113: 755-765

Fishell G, Kriegstein AR (2003) Neurons from radial glia: the consequences of asymmetric inheritance. Curr Opin Neurobiol 13: 34-41

Forscher P, Smith SJ (1988) Actions of cytochalasins on the organization of actin filaments and microtubules in a neuronal growth cone. J Cell Biol 107: 1505-1516

Freemont PS (1993) The RING finger. A novel protein sequence motif related to the zinc finger. Ann N Y Acad Sci 684: 174-192

Gao FB, Kohwi M, Brenman JE, Jan LY, Jan YN (2000) Control of dendritic field formation in Drosophila: the roles of flamingo and competition between homologous neurons. Neuron 28: $91-101$

Garbe DS, Bashaw GJ (2004) Axon guidance at the midline: from mutants to mechanisms. Crit Rev Biochem Mol Biol 39: 319-341

Gaudilliere B, Konishi Y, de la Iglesia N, Yao G, Bonni A (2004) A CaMKII-NeuroD signaling pathway specifies dendritic morphogenesis. Neuron 41: 229-241

Gaughan L, Brady ME, Cook S, Neal DE, Robson CN (2001) Tip60 is a co-activator specific for class I nuclear hormone receptors. J Biol Chem 276: 46841-46848

Georges PC, Hadzimichalis NM, Sweet ES, Firestein BL (2008) The yin-yang of dendrite morphology: unity of actin and microtubules. Mol Neurobiol 38: 270-284

Giaccia AJ, Kastan MB (1998) The complexity of p53 modulation: emerging patterns from divergent signals. Genes Dev 12: 2973-2983

Gieffers C, Peters BH, Kramer ER, Dotti CG, Peters JM (1999) Expression of the CDH1associated form of the anaphase-promoting complex in postmitotic neurons. Proc Natl Acad Sci U S A 96: 11317-11322 
Glebova NO, Ginty DD (2004) Heterogeneous requirement of NGF for sympathetic target innervation in vivo. J Neurosci 24: 743-751

Goto E, Yamanaka Y, Ishikawa A, Aoki-Kawasumi M, Mito-Yoshida M, Ohmura-Hoshino M, Matsuki Y, Kajikawa M, Hirano H, Ishido S (2010) Contribution of lysine 11-linked ubiquitination to MIR2-mediated major histocompatibility complex class I internalization. $J$ Biol Chem 285: 35311-35319

Grueber WB, Sagasti A (2010) Self-avoidance and tiling: Mechanisms of dendrite and axon spacing. Cold Spring Harb Perspect Biol 2: a001750

Guenette S, Chang Y, Hiesberger T, Richardson JA, Eckman CB, Eckman EA, Hammer RE, Herz J (2006) Essential roles for the FE65 amyloid precursor protein-interacting proteins in brain development. EMBO J 25: 420-431

Guenette SY, Chen J, Ferland A, Haass C, Capell A, Tanzi RE (1999) hFE65L influences amyloid precursor protein maturation and secretion. J Neurochem 73: 985-993

Guenette SY, Chen J, Jondro PD, Tanzi RE (1996) Association of a novel human FE65-like protein with the cytoplasmic domain of the beta-amyloid precursor protein. Proc Natl Acad Sci U S A 93: 10832-10837

Gundersen RW, Barrett JN (1979) Neuronal chemotaxis: chick dorsal-root axons turn toward high concentrations of nerve growth factor. Science 206: 1079-1080

Guo X, Rueger D, Higgins D (1998) Osteogenic protein-1 and related bone morphogenetic proteins regulate dendritic growth and the expression of microtubule-associated protein-2 in rat sympathetic neurons. Neurosci Lett 245: 131-134

Hall A (1998) Rho GTPases and the actin cytoskeleton. Science 279: 509-514

Hamburger V, Levi-Montalcini R (1949) Proliferation, differentiation and degeneration in the spinal ganglia of the chick embryo under normal and experimental conditions. $J$ Exp Zool 111: 457-501

Hand R, Bortone D, Mattar P, Nguyen L, Heng JI, Guerrier S, Boutt E, Peters E, Barnes AP, Parras C, Schuurmans C, Guillemot F, Polleux F (2005) Phosphorylation of Neurogenin2 specifies the migration properties and the dendritic morphology of pyramidal neurons in the neocortex. Neuron 48: 45-62

Harada A, Teng J, Takei Y, Oguchi K, Hirokawa N (2002) MAP2 is required for dendrite elongation, PKA anchoring in dendrites, and proper PKA signal transduction. J Cell Biol 158: $541-549$

Hashimoto Y, Ito Y, Niikura T, Shao Z, Hata M, Oyama F, Nishimoto I (2001a) Mechanisms of neuroprotection by a novel rescue factor humanin from Swedish mutant amyloid precursor protein. Biochem Biophys Res Commun 283: 460-468 
Hashimoto Y, Niikura T, Ito Y, Sudo H, Hata M, Arakawa E, Abe Y, Kita Y, Nishimoto I (2001b) Detailed characterization of neuroprotection by a rescue factor humanin against various Alzheimer's disease-relevant insults. J Neurosci 21: 9235-9245

Hashimoto Y, Niikura T, Tajima H, Yasukawa T, Sudo H, Ito Y, Kita Y, Kawasumi M, Kouyama K, Doyu M, Sobue G, Koide T, Tsuji S, Lang J, Kurokawa K, Nishimoto I (2001c) A rescue factor abolishing neuronal cell death by a wide spectrum of familial Alzheimer's disease genes and Abeta. Proc Natl Acad Sci U S A 98: 6336-6341

Hass MR, Yankner BA (2005) A \{gamma\}-secretase-independent mechanism of signal transduction by the amyloid precursor protein.J Biol Chem 280: 36895-36904

Hatten ME (1999) Central nervous system neuronal migration. Annu Rev Neurosci 22: 511539

He L, Lu XY, Jolly AF, Eldridge AG, Watson SJ, Jackson PK, Barsh GS, Gunn TM (2003) Spongiform degeneration in mahoganoid mutant mice. Science 299: 710-712

Hemmati-Brivanlou A, Kelly OG, Melton DA (1994) Follistatin, an antagonist of activin, is expressed in the Spemann organizer and displays direct neuralizing activity. Cell 77: 283295

Herms J, Anliker B, Heber S, Ring S, Fuhrmann M, Kretzschmar H, Sisodia S, Muller U (2004) Cortical dysplasia resembling human type 2 lissencephaly in mice lacking all three APP family members. EMBO J 23: 4106-4115

Hershko A, Ciechanover A (1998) The ubiquitin system. Annu Rev Biochem 67: 425-479

Hirose M, Ishizaki T, Watanabe N, Uehata M, Kranenburg O, Moolenaar WH, Matsumura F, Maekawa M, Bito H, Narumiya S (1998) Molecular dissection of the Rho-associated protein kinase (p160ROCK)-regulated neurite remodeling in neuroblastoma N1E-115 cells. J Cell Biol 141: 1625-1636

Hoe HS, Magill LA, Guenette S, Fu Z, Vicini S, Rebeck GW (2006) FE65 interaction with the ApoE receptor ApoEr2.J Biol Chem 281: 24521-24530

Holloway RL, Jr. (1966) Dendritic branching: some preliminary results of training and complexity in rat visual cortex. Brain Res 2: 393-396

Hoogenraad CC, Milstein AD, Ethell IM, Henkemeyer M, Sheng M (2005) GRIP1 controls dendrite morphogenesis by regulating EphB receptor trafficking. Nat Neurosci 8: 906-915

Howell BW, Herrick TM, Cooper JA (1999) Reelin-induced tyrosine [corrected] phosphorylation of disabled 1 during neuronal positioning. Genes Dev 13: 643-648

Howell BW, Herrick TM, Hildebrand JD, Zhang Y, Cooper JA (2000) Dab1 tyrosine phosphorylation sites relay positional signals during mouse brain development. Curr Biol 10: $877-885$ 
Hsu YT, Wolter KG, Youle RJ (1997) Cytosol-to-membrane redistribution of Bax and BclX(L) during apoptosis. Proc Natl Acad Sci U S A 94: 3668-3672

Hu Q, Hearn MG, Jin LW, Bressler SL, Martin GM (1999) Alternatively spliced isoforms of FE65 serve as neuron-specific and non-neuronal markers. J Neurosci Res 58: 632-640

Huang L, Kinnucan E, Wang G, Beaudenon S, Howley PM, Huibregtse JM, Pavletich NP (1999) Structure of an E6AP-UbcH7 complex: insights into ubiquitination by the E2-E3 enzyme cascade. Science 286: 1321-1326

Huber AB, Kolodkin AL, Ginty DD, Cloutier JF (2003) Signaling at the growth cone: ligandreceptor complexes and the control of axon growth and guidance. Annu Rev Neurosci 26: 509-563

Hughes ME, Bortnick R, Tsubouchi A, Baumer P, Kondo M, Uemura T, Schmucker D (2007) Homophilic Dscam interactions control complex dendrite morphogenesis. Neuron 54: 417427

Ikeuchi Y, Stegmuller J, Netherton S, Huynh MA, Masu M, Frank D, Bonni S, Bonni A (2009) A SnoN-Ccd1 pathway promotes axonal morphogenesis in the mammalian brain. J Neurosci 29: $4312-4321$

Ikin AF, Sabo SL, Lanier LM, Buxbaum JD (2007) A macromolecular complex involving the amyloid precursor protein (APP) and the cytosolic adapter FE65 is a negative regulator of axon branching. Mol Cell Neurosci 35: 57-63

Ikura T, Ogryzko VV, Grigoriev M, Groisman R, Wang J, Horikoshi M, Scully R, Qin J, Nakatani Y (2000) Involvement of the TIP60 histone acetylase complex in DNA repair and apoptosis. Cell 102: 463-473

Imai Y, Soda M, Hatakeyama S, Akagi T, Hashikawa T, Nakayama KI, Takahashi R (2002) CHIP is associated with Parkin, a gene responsible for familial Parkinson's disease, and enhances its ubiquitin ligase activity. Mol Cell 10: 55-67

Ip YT, Davis RJ (1998) Signal transduction by the c-Jun N-terminal kinase (JNK)--from inflammation to development. Curr Opin Cell Biol 10: 205-219

Ito T, Niwa J, Hishikawa N, Ishigaki S, Doyu M, Sobue G (2003) Dorfin localizes to Lewy bodies and ubiquitylates synphilin-1. J Biol Chem 278: 29106-29114

Jan YN, Jan LY (2003) The control of dendrite development. Neuron 40: 229-242

Jeffers JR, Parganas E, Lee Y, Yang C, Wang J, Brennan J, MacLean KH, Han J, Chittenden T, Ihle JN, McKinnon PJ, Cleveland JL, Zambetti GP (2003) Puma is an essential mediator of p53-dependent and -independent apoptotic pathways. Cancer Cell 4: 321-328

Jenner P (2003) Oxidative stress in Parkinson's disease. Ann Neurol 53 Suppl 3: S26-36; discussion S36-28 
Jiang X, Wang X (2000) Cytochrome c promotes caspase-9 activation by inducing nucleotide binding to Apaf-1. J Biol Chem 275: 31199-31203

Jiao J, Sun K, Walker WP, Bagher P, Cota CD, Gunn TM (2009) Abnormal regulation of TSG101 in mice with spongiform neurodegeneration. Biochim Biophys Acta 1792: 10271035

Johnson CD, Esquela-Kerscher A, Stefani G, Byrom M, Kelnar K, Ovcharenko D, Wilson M, Wang X, Shelton J, Shingara J, Chin L, Brown D, Slack FJ (2007) The let-7 microRNA represses cell proliferation pathways in human cells. Cancer Res 67: 7713-7722

Jossin Y, Goffinet AM (2007) Reelin signals through phosphatidylinositol 3-kinase and Akt to control cortical development and through mTor to regulate dendritic growth. Mol Cell Biol 27: 7113-7124

Kawabe H, Brose N (2011) The role of ubiquitylation in nerve cell development. Nat Rev Neurosci 12: 251-268

Kawabe H, Neeb A, Dimova K, Young SM, Jr., Takeda M, Katsurabayashi S, Mitkovski M, Malakhova OA, Zhang DE, Umikawa M, Kariya K, Goebbels S, Nave KA, Rosenmund C, Jahn 0, Rhee J, Brose N (2010) Regulation of Rap2A by the ubiquitin ligase Nedd4-1 controls neurite development. Neuron 65: 358-372

Kennedy TE (2000) Cellular mechanisms of netrin function: long-range and short-range actions. Biochem Cell Biol 78: 569-575

Kerr JF, Wyllie AH, Currie AR (1972) Apoptosis: a basic biological phenomenon with wideranging implications in tissue kinetics. Br J Cancer 26: 239-257

Kesavapany S, Banner SJ, Lau KF, Shaw CE, Miller CC, Cooper JD, McLoughlin DM (2002) Expression of the Fe65 adapter protein in adult and developing mouse brain. Neuroscience 115: $951-960$

Kim AH, Puram SV, Bilimoria PM, Ikeuchi Y, Keough S, Wong M, Rowitch D, Bonni A (2009) A centrosomal Cdc20-APC pathway controls dendrite morphogenesis in postmitotic neurons. Cell 136: 322-336

Kim BY, Olzmann JA, Barsh GS, Chin LS, Li L (2007a) Spongiform neurodegenerationassociated E3 ligase Mahogunin ubiquitylates TSG101 and regulates endosomal trafficking. Mol Biol Cell 18: 1129-1142

Kim JB, Zaehres H, Wu G, Gentile L, Ko K, Sebastiano V, Arauzo-Bravo MJ, Ruau D, Han DW, Zenke M, Scholer HR (2008) Pluripotent stem cells induced from adult neural stem cells by reprogramming with two factors. Nature 454: 646-650

Kim SY, Kim MY, Mo JS, Park HS (2007b) Notch1 intracellular domain suppresses APP intracellular domain-Tip60-Fe65 complex mediated signaling through physical interaction. Biochim Biophys Acta 1773: 736-746 
Kimberly WT, Zheng JB, Guenette SY, Selkoe DJ (2001) The intracellular domain of the betaamyloid precursor protein is stabilized by Fe65 and translocates to the nucleus in a notchlike manner. J Biol Chem 276: 40288-40292

Kinoshita A, Whelan CM, Berezovska O, Hyman BT (2002a) The gamma secretasegenerated carboxyl-terminal domain of the amyloid precursor protein induces apoptosis via Tip60 in H4 cells. J Biol Chem 277: 28530-28536

Kinoshita A, Whelan CM, Smith CJ, Berezovska 0, Hyman BT (2002b) Direct visualization of the gamma secretase-generated carboxyl-terminal domain of the amyloid precursor protein: association with Fe65 and translocation to the nucleus. J Neurochem 82: 839-847

Kirilly D, Gu Y, Huang Y, Wu Z, Bashirullah A, Low BC, Kolodkin AL, Wang H, Yu F (2009) A genetic pathway composed of Sox14 and Mical governs severing of dendrites during pruning. Nat Neurosci 12: 1497-1505

Kischkel FC, Hellbardt S, Behrmann I, Germer M, Pawlita M, Krammer PH, Peter ME (1995) Cytotoxicity-dependent APO-1 (Fas/CD95)-associated proteins form a death-inducing signaling complex (DISC) with the receptor. EMBO J 14: 5579-5588

Kischkel FC, Lawrence DA, Tinel A, LeBlanc H, Virmani A, Schow P, Gazdar A, Blenis J, Arnott D, Ashkenazi A (2001) Death receptor recruitment of endogenous caspase-10 and apoptosis initiation in the absence of caspase-8. J Biol Chem 276: 46639-46646

Ko LJ, Prives C (1996) p53: puzzle and paradigm. Genes Dev 10: 1054-1072

Kocherhans S, Madhusudan A, Doehner J, Breu KS, Nitsch RM, Fritschy JM, Knuesel I (2010) Reduced Reelin expression accelerates amyloid-beta plaque formation and tau pathology in transgenic Alzheimer's disease mice. J Neurosci 30: 9228-9240

Komander D, Rape M (2012) The ubiquitin code. Annu Rev Biochem 81: 203-229

Konishi Y, Stegmuller J, Matsuda T, Bonni S, Bonni A (2004) Cdh1-APC controls axonal growth and patterning in the mammalian brain. Science 303: 1026-1030

Kullander K, Klein R (2002) Mechanisms and functions of Eph and ephrin signalling. Nat Rev Mol Cell Biol 3: 475-486

Lamb TM, Knecht AK, Smith WC, Stachel SE, Economides AN, Stahl N, Yancopolous GD, Harland RM (1993) Neural induction by the secreted polypeptide noggin. Science 262: 713718

Lanier LM, Gates MA, Witke W, Menzies AS, Wehman AM, Macklis JD, Kwiatkowski D, Soriano P, Gertler FB (1999) Mena is required for neurulation and commissure formation. Neuron 22: 313-325

Lasorella A, Stegmuller J, Guardavaccaro D, Liu G, Carro MS, Rothschild G, de la TorreUbieta L, Pagano M, Bonni A, Iavarone A (2006) Degradation of Id2 by the anaphasepromoting complex couples cell cycle exit and axonal growth. Nature 442: 471-474 
Lassot I, Robbins I, Kristiansen M, Rahmeh R, Jaudon F, Magiera MM, Mora S, Vanhille L, Lipkin A, Pettmann B, Ham J, Desagher S (2010) Trim17, a novel E3 ubiquitin-ligase, initiates neuronal apoptosis. Cell Death Differ 17: 1928-1941

Lau KF, Chan WM, Perkinton MS, Tudor EL, Chang RC, Chan HY, McLoughlin DM, Miller CC (2008) Dexras1 interacts with FE65 to regulate FE65-amyloid precursor proteindependent transcription. J Biol Chem 283: 34728-34737

Le Roux P, Behar S, Higgins D, Charette M (1999) OP-1 enhances dendritic growth from cerebral cortical neurons in vitro. Exp Neurol 160: 151-163

Lee EJ, Hyun S, Chun J, Shin SH, Kang SS (2009a) Ubiquitylation of Fe65 adaptor protein by neuronal precursor cell expressed developmentally down regulated 4-2 (Nedd4-2) via the WW domain interaction with Fe65. Exp Mol Med 41: 555-568

Lee HH, Jan LY, Jan YN (2009b) Drosophila IKK-related kinase Ik2 and Katanin p60-like 1 regulate dendrite pruning of sensory neuron during metamorphosis. Proc Natl Acad Sci U S A 106: 6363-6368

Lee KJ, Jessell TM (1999) The specification of dorsal cell fates in the vertebrate central nervous system. Annu Rev Neurosci 22: 261-294

Lee T, Winter C, Marticke SS, Lee A, Luo L (2000) Essential roles of Drosophila RhoA in the regulation of neuroblast proliferation and dendritic but not axonal morphogenesis. Neuron 25: $307-316$

Lemercier C, Legube G, Caron C, Louwagie M, Garin J, Trouche D, Khochbin S (2003) Tip60 acetyltransferase activity is controlled by phosphorylation. J Biol Chem 278: 4713-4718

Letourneau PC (1978) Chemotactic response of nerve fiber elongation to nerve growth factor. Dev Biol 66: 183-196

Levine B, Yuan J (2005) Autophagy in cell death: an innocent convict? J Clin Invest 115: 2679-2688

Li LY, Luo X, Wang X (2001) Endonuclease G is an apoptotic DNase when released from mitochondria. Nature 412: 95-99

Li M, Brooks CL, Wu-Baer F, Chen D, Baer R, Gu W (2003) Mono- versus polyubiquitination: differential control of p53 fate by Mdm2. Science 302: 1972-1975

Li N, Zhao CT, Wang Y, Yuan XB (2010) The transcription factor Cux1 regulates dendritic morphology of cortical pyramidal neurons. PLoS One 5: e10596

Li P, Nijhawan D, Budihardjo I, Srinivasula SM, Ahmad M, Alnemri ES, Wang X (1997) Cytochrome $c$ and dATP-dependent formation of Apaf-1/caspase-9 complex initiates an apoptotic protease cascade. Cell 91: 479-489 
Litterman N, Ikeuchi Y, Gallardo G, O'Connell BC, Sowa ME, Gygi SP, Harper JW, Bonni A (2011) An OBSL1-Cul7Fbxw8 ubiquitin ligase signaling mechanism regulates Golgi morphology and dendrite patterning. PLoS Biol 9: e1001060

Liu QY, Lei JX, Sikorska M, Liu R (2008) A novel brain-enriched E3 ubiquitin ligase RNF182 is up regulated in the brains of Alzheimer's patients and targets ATP6V0C for degradation. Mol Neurodegener 3: 4

Liu X, Kim CN, Yang J, Jemmerson R, Wang X (1996) Induction of apoptotic program in cellfree extracts: requirement for dATP and cytochrome c. Cell 86: 147-157

Liu Z, Steward R, Luo L (2000) Drosophila Lis1 is required for neuroblast proliferation, dendritic elaboration and axonal transport. Nat Cell Biol 2: 776-783

Lossi L, Merighi A (2003) In vivo cellular and molecular mechanisms of neuronal apoptosis in the mammalian CNS. Prog Neurobiol 69: 287-312

Lunn ER, Perry VH, Brown MC, Rosen H, Gordon S (1989) Absence of Wallerian Degeneration does not Hinder Regeneration in Peripheral Nerve. Eur J Neurosci 1: 27-33

Luo L (2000) Rho GTPases in neuronal morphogenesis. Nat Rev Neurosci 1: 173-180

Luo L (2002) Actin cytoskeleton regulation in neuronal morphogenesis and structural plasticity. Annu Rev Cell Dev Biol 18: 601-635

Luo L, O'Leary DD (2005) Axon retraction and degeneration in development and disease. Annu Rev Neurosci 28: 127-156

Lupo G, Harris WA, Lewis KE (2006) Mechanisms of ventral patterning in the vertebrate nervous system. Nat Rev Neurosci 7: 103-114

Lyuksyutova AI, Lu CC, Milanesio N, King LA, Guo N, Wang Y, Nathans J, Tessier-Lavigne M, Zou Y (2003) Anterior-posterior guidance of commissural axons by Wnt-frizzled signaling. Science 302: 1984-1988

MacEwan DJ (2002) TNF receptor subtype signalling: differences and cellular consequences. Cell Signal 14: 477-492

Mack TG, Reiner M, Beirowski B, Mi W, Emanuelli M, Wagner D, Thomson D, Gillingwater T, Court F, Conforti L, Fernando FS, Tarlton A, Andressen C, Addicks K, Magni G, Ribchester RR, Perry VH, Coleman MP (2001) Wallerian degeneration of injured axons and synapses is delayed by a Ube4b/Nmnat chimeric gene. Nat Neurosci 4: 1199-1206

Martin DP, Schmidt RE, DiStefano PS, Lowry OH, Carter JG, Johnson EM, Jr. (1988) Inhibitors of protein synthesis and RNA synthesis prevent neuronal death caused by nerve growth factor deprivation.J Cell Biol 106: 829-844

Mata IF, Lockhart PJ, Farrer MJ (2004) Parkin genetics: one model for Parkinson's disease. Hum Mol Genet 13 Spec No 1: R127-133 
McAllister AK (2000) Cellular and molecular mechanisms of dendrite growth. Cereb Cortex 10: $963-973$

McAllister AK, Lo DC, Katz LC (1995) Neurotrophins regulate dendritic growth in developing visual cortex. Neuron 15: 791-803

McConnell SK (1995) Constructing the cerebral cortex: neurogenesis and fate determination. Neuron 15: 761-768

McLoughlin DM, Miller CC (2008) The FE65 proteins and Alzheimer's disease. J Neurosci Res 86: 744-754

McNaught KS, Mytilineou C, Jnobaptiste R, Yabut J, Shashidharan P, Jennert P, Olanow CW (2002) Impairment of the ubiquitin-proteasome system causes dopaminergic cell death and inclusion body formation in ventral mesencephalic cultures. J Neurochem 81: 301-306

Medema JP, Scaffidi C, Kischkel FC, Shevchenko A, Mann M, Krammer PH, Peter ME (1997) FLICE is activated by association with the CD95 death-inducing signaling complex (DISC). EMBO J 16: 2794-2804

Metzstein MM, Stanfield GM, Horvitz HR (1998) Genetics of programmed cell death in C. elegans: past, present and future. Trends Genet 14: 410-416

Micheau O, Tschopp J (2003) Induction of TNF receptor I-mediated apoptosis via two sequential signaling complexes. Cell 114: 181-190

Miller KA, Gunn TM, Carrasquillo MM, Lamoreux ML, Galbraith DB, Barsh GS (1997) Genetic studies of the mouse mutations mahogany and mahoganoid. Genetics 146: 14071415

Minopoli G, de Candia P, Bonetti A, Faraonio R, Zambrano N, Russo T (2001) The betaamyloid precursor protein functions as a cytosolic anchoring site that prevents Fe65 nuclear translocation. J Biol Chem 276: 6545-6550

Miravalle L, Calero M, Takao M, Roher AE, Ghetti B, Vidal R (2005) Amino-terminally truncated Abeta peptide species are the main component of cotton wool plaques. Biochemistry 44: 10810-10821

Moore DL, Goldberg JL (2011) Multiple transcription factor families regulate axon growth and regeneration. Dev Neurobiol 71: 1186-1211

Mukaetova-Ladinska EB, Arnold H, Jaros E, Perry R, Perry E (2004) Depletion of MAP2 expression and laminar cytoarchitectonic changes in dorsolateral prefrontal cortex in adult autistic individuals. Neuropathol Appl Neurobiol 30: 615-623

Nakaya T, Suzuki T (2006) Role of APP phosphorylation in FE65-dependent gene transactivation mediated by AICD. Genes Cells 11: 633-645 
Nateri AS, Riera-Sans L, Da Costa C, Behrens A (2004) The ubiquitin ligase SCFFbw7 antagonizes apoptotic JNK signaling. Science 303: 1374-1378

Nechushtan A, Smith CL, Lamensdorf I, Yoon SH, Youle RJ (2001) Bax and Bak coalesce into novel mitochondria-associated clusters during apoptosis. J Cell Biol 153: 1265-1276

Nedivi E, Wu GY, Cline HT (1998) Promotion of dendritic growth by CPG15, an activityinduced signaling molecule. Science 281: 1863-1866

Nikolaev A, McLaughlin T, O'Leary DD, Tessier-Lavigne M (2009) APP binds DR6 to trigger axon pruning and neuron death via distinct caspases. Nature 457: 981-989

Nishitani H, Sugimoto N, Roukos V, Nakanishi Y, Saijo M, Obuse C, Tsurimoto T, Nakayama KI, Nakayama K, Fujita M, Lygerou Z, Nishimoto T (2006) Two E3 ubiquitin ligases, SCFSkp2 and DDB1-Cul4, target human Cdt1 for proteolysis. EMBO J 25: 1126-1136

Noctor SC, Flint AC, Weissman TA, Dammerman RS, Kriegstein AR (2001) Neurons derived from radial glial cells establish radial units in neocortex. Nature 409: 714-720

Noctor SC, Martinez-Cerdeno V, Ivic L, Kriegstein AR (2004) Cortical neurons arise in symmetric and asymmetric division zones and migrate through specific phases. Nat Neurosci 7: 136-144

Olave I, Wang W, Xue Y, Kuo A, Crabtree GR (2002) Identification of a polymorphic, neuronspecific chromatin remodeling complex. Genes Dev 16: 2509-2517

Oltvai ZN, Milliman CL, Korsmeyer SJ (1993) Bcl-2 heterodimerizes in vivo with a conserved homolog, Bax, that accelerates programmed cell death. Cell 74: 609-619

Oppenheim RW (1991) Cell death during development of the nervous system. Annu Rev Neurosci 14: 453-501

Palazzo AF, Cook TA, Alberts AS, Gundersen GG (2001) mDia mediates Rho-regulated formation and orientation of stable microtubules. Nat Cell Biol 3: 723-729

Parrish JZ, Emoto K, Kim MD, Jan YN (2007) Mechanisms that regulate establishment, maintenance, and remodeling of dendritic fields. Annu Rev Neurosci 30: 399-423

Parrish JZ, Kim MD, Jan LY, Jan YN (2006) Genome-wide analyses identify transcription factors required for proper morphogenesis of Drosophila sensory neuron dendrites. Genes Dev 20: 820-835

Passer B, Pellegrini L, Russo C, Siegel RM, Lenardo MJ, Schettini G, Bachmann M, Tabaton M, D'Adamio L (2000) Generation of an apoptotic intracellular peptide by gamma-secretase cleavage of Alzheimer's amyloid beta protein precursor. J Alzheimers Dis 2: 289-301

Pasterkamp RJ, Kolodkin AL (2003) Semaphorin junction: making tracks toward neural connectivity. Curr Opin Neurobiol 13: 79-89 
Peng J, Schwartz D, Elias JE, Thoreen CC, Cheng D, Marsischky G, Roelofs J, Finley D, Gygi SP (2003) A proteomics approach to understanding protein ubiquitination. Nat Biotechnol 21: 921-926

Peng Y, Laser J, Shi G, Mittal K, Melamed J, Lee P, Wei JJ (2008) Antiproliferative effects by Let-7 repression of high-mobility group A2 in uterine leiomyoma. Mol Cancer Res 6: 663673

Perkinton MS, Standen CL, Lau KF, Kesavapany S, Byers HL, Ward M, McLoughlin DM, Miller CC (2004) The c-Abl tyrosine kinase phosphorylates the Fe65 adaptor protein to stimulate Fe65/amyloid precursor protein nuclear signaling. J Biol Chem 279: 22084-22091

Perutz MF, Windle AH (2001) Cause of neural death in neurodegenerative diseases attributable to expansion of glutamine repeats. Nature 412: 143-144

Petroski MD, Deshaies RJ (2005) Mechanism of lysine 48-linked ubiquitin-chain synthesis by the cullin-RING ubiquitin-ligase complex SCF-Cdc34. Cell 123: 1107-1120

Petrucelli L, Dawson TM (2004) Mechanism of neurodegenerative disease: role of the ubiquitin proteasome system. Ann Med 36: 315-320

Petrucelli L, O'Farrell C, Lockhart PJ, Baptista M, Kehoe K, Vink L, Choi P, Wolozin B, Farrer M, Hardy J, Cookson MR (2002) Parkin protects against the toxicity associated with mutant alpha-synuclein: proteasome dysfunction selectively affects catecholaminergic neurons. Neuron 36: 1007-1019

Phan LK, Lin F, LeDuc CA, Chung WK, Leibel RL (2002) The mouse mahoganoid coat color mutation disrupts a novel C3HC4 RING domain protein. J Clin Invest 110: 1449-1459

Phillips RJ (1963) New mutant: non-agouti curly. Mouse News Letter

Piccolo S, Agius E, Leyns L, Bhattacharyya S, Grunz H, Bouwmeester T, De Robertis EM (1999) The head inducer Cerberus is a multifunctional antagonist of Nodal, BMP and Wnt signals. Nature 397: 707-710

Piccolo S, Sasai Y, Lu B, De Robertis EM (1996) Dorsoventral patterning in Xenopus: inhibition of ventral signals by direct binding of chordin to BMP-4. Cell 86: 589-598

Polleux F, Giger RJ, Ginty DD, Kolodkin AL, Ghosh A (1998) Patterning of cortical efferent projections by semaphorin-neuropilin interactions. Science 282: 1904-1906

Polleux F, Ince-Dunn G, Ghosh A (2007) Transcriptional regulation of vertebrate axon guidance and synapse formation. Nat Rev Neurosci 8: 331-340

Polleux F, Morrow T, Ghosh A (2000) Semaphorin 3A is a chemoattractant for cortical apical dendrites. Nature 404: 567-573

Portelius E, Bogdanovic N, Gustavsson MK, Volkmann I, Brinkmalm G, Zetterberg H, Winblad B, Blennow K (2010) Mass spectrometric characterization of brain amyloid beta 
isoform signatures in familial and sporadic Alzheimer's disease. Acta Neuropathol 120: 185-193

Qian X, Shen Q, Goderie SK, He W, Capela A, Davis AA, Temple S (2000) Timing of CNS cell generation: a programmed sequence of neuron and glial cell production from isolated murine cortical stem cells. Neuron 28: 69-80

Quinn JC, Molinek M, Martynoga BS, Zaki PA, Faedo A, Bulfone A, Hevner RF, West JD, Price DJ (2007) Pax6 controls cerebral cortical cell number by regulating exit from the cell cycle and specifies cortical cell identity by a cell autonomous mechanism. Dev Biol 302: 50-65

Radzimanowski J, Simon B, Sattler M, Beyreuther K, Sinning I, Wild K (2008) Structure of the intracellular domain of the amyloid precursor protein in complex with Fe65-PTB2. EMBO Rep 9: 1134-1140

Rakic P (1971) Guidance of neurons migrating to the fetal monkey neocortex. Brain Res 33: 471-476

Rakic P (1972) Mode of cell migration to the superficial layers of fetal monkey neocortex. $J$ Comp Neurol 145: 61-83

Ramón y Cajal S (1911) Histology of the nervous system of man and vertebrates, (1995 translation).

Raymond GV, Bauman ML, Kemper TL (1996) Hippocampus in autism: a Golgi analysis. Acta Neuropathol 91: 117-119

Redmond L, Oh SR, Hicks C, Weinmaster G, Ghosh A (2000) Nuclear Notch1 signaling and the regulation of dendritic development. Nat Neurosci 3: 30-40

Rotin D, Kumar S (2009) Physiological functions of the HECT family of ubiquitin ligases. Nat Rev Mol Cell Biol 10: 398-409

Sabo SL, Ikin AF, Buxbaum JD, Greengard P (2001) The Alzheimer amyloid precursor protein (APP) and FE65, an APP-binding protein, regulate cell movement. J Cell Biol 153: $1403-1414$

Sabo SL, Ikin AF, Buxbaum JD, Greengard P (2003) The amyloid precursor protein and its regulatory protein, FE65, in growth cones and synapses in vitro and in vivo. J Neurosci 23: 5407-5415

Sahara N, Murayama M, Mizoroki T, Urushitani M, Imai Y, Takahashi R, Murata S, Tanaka K, Takashima A (2005) In vivo evidence of CHIP up-regulation attenuating tau aggregation. $J$ Neurochem 94: 1254-1263

Saito T, Suemoto T, Brouwers N, Sleegers K, Funamoto S, Mihira N, Matsuba Y, Yamada K, Nilsson P, Takano J, Nishimura M, Iwata N, Van Broeckhoven C, Ihara Y, Saido TC (2011) Potent amyloidogenicity and pathogenicity of Abeta43. Nat Neurosci 14: 1023-1032 
Saleh A, Srinivasula SM, Acharya S, Fishel R, Alnemri ES (1999) Cytochrome c and dATPmediated oligomerization of Apaf- 1 is a prerequisite for procaspase-9 activation. J Biol Chem 274: 17941-17945

Santiard-Baron D, Langui D, Delehedde M, Delatour B, Schombert B, Touchet N, Tremp G, Paul MF, Blanchard V, Sergeant N, Delacourte A, Duyckaerts C, Pradier L, Mercken L (2005) Expression of human FE65 in amyloid precursor protein transgenic mice is associated with a reduction in beta-amyloid load. J Neurochem 93: 330-338

Sasal Y, Lu B, Steinbelsser H, De Robertis EM (1995) Regulation of neural induction by the Chd and Bmp-4 antagonistic patterning signals in Xenopus. Nature 378: 419

Scaffidi C, Medema JP, Krammer PH, Peter ME (1997) FLICE is predominantly expressed as two functionally active isoforms, caspase-8/a and caspase-8/b. J Biol Chem 272: 2695326958

Scheel H, Hofmann K (2003) No evidence for PHD fingers as ubiquitin ligases. Trends Cell Biol 13: 285-287; author reply 287-288

Schmahl W, Knoedlseder M, Favor J, Davidson D (1993) Defects of neuronal migration and the pathogenesis of cortical malformations are associated with Small eye (Sey) in the mouse, a point mutation at the Pax-6-locus. Acta Neuropathol 86: 126-135

Schnell JD, Hicke L (2003) Non-traditional functions of ubiquitin and ubiquitin-binding proteins. J Biol Chem 278: 35857-35860

Schoenmann Z, Assa-Kunik E, Tiomny S, Minis A, Haklai-Topper L, Arama E, Yaron A (2010) Axonal degeneration is regulated by the apoptotic machinery or a NAD+-sensitive pathway in insects and mammals. J Neurosci 30: 6375-6386

Schulz JB, Dichgans J (1999) Molecular pathogenesis of movement disorders: are protein aggregates a common link in neuronal degeneration? Curr Opin Neurol 12: 433-439

Schutze S, Tchikov V, Schneider-Brachert W (2008) Regulation of TNFR1 and CD95 signalling by receptor compartmentalization. Nat Rev Mol Cell Biol 9: 655-662

Scott EK, Luo L (2001) How do dendrites take their shape? Nat Neurosci 4: 359-365

Selkoe DJ (1989) Amyloid beta protein precursor and the pathogenesis of Alzheimer's disease. Cell 58: 611-612

Selkoe DJ (1998) The cell biology of beta-amyloid precursor protein and presenilin in Alzheimer's disease. Trends Cell Biol 8: 447-453

Sempere LF, Freemantle S, Pitha-Rowe I, Moss E, Dmitrovsky E, Ambros V (2004) Expression profiling of mammalian microRNAs uncovers a subset of brain-expressed microRNAs with possible roles in murine and human neuronal differentiation. Genome Biol 5: R13 
Sestan N, Artavanis-Tsakonas S, Rakic P (1999) Contact-dependent inhibition of cortical neurite growth mediated by notch signaling. Science 286: 741-746

Shimura H, Schwartz D, Gygi SP, Kosik KS (2004) CHIP-Hsc70 complex ubiquitinates phosphorylated tau and enhances cell survival. J Biol Chem 279: 4869-4876

Sidman RL, Rakic P (1973) Neuronal migration, with special reference to developing human brain: a review. Brain Res 62: 1-35

Smith CJ, Watson JD, VanHoven MK, Colon-Ramos DA, Miller DM, 3rd (2012) Netrin (UNC6) mediates dendritic self-avoidance. Nat Neurosci 15: 731-737

Smith MA, Rottkamp CA, Nunomura A, Raina AK, Perry G (2000) Oxidative stress in Alzheimer's disease. Biochim Biophys Acta 1502: 139-144

Song HJ, Ming GL, Poo MM (1997) cAMP-induced switching in turning direction of nerve growth cones. Nature 388: 275-279

Spillantini MG, Schmidt ML, Lee VM, Trojanowski JQ, Jakes R, Goedert M (1997) Alphasynuclein in Lewy bodies. Nature 388: 839-840

Sproul AA, Xu Z, Wilhelm M, Gire S, Greene LA (2009) Cbl negatively regulates JNK activation and cell death. Cell Res 19: 950-961

Srinivasula SM, Ahmad M, Fernandes-Alnemri T, Alnemri ES (1998) Autoactivation of procaspase- 9 by Apaf-1-mediated oligomerization. Mol Cell 1: 949-957

Stante M, Minopoli G, Passaro F, Raia M, Vecchio LD, Russo T (2009) Fe65 is required for Tip60-directed histone H4 acetylation at DNA strand breaks. Proc Natl Acad Sci U S A 106: 5093-5098

Stegmuller J, Bonni A (2005) Moving past proliferation: new roles for Cdh1-APC in postmitotic neurons. Trends Neurosci 28: 596-601

Stegmuller J, Huynh MA, Yuan Z, Konishi Y, Bonni A (2008) TGFbeta-Smad2 signaling regulates the Cdh1-APC/SnoN pathway of axonal morphogenesis. J Neurosci 28: 1961-1969

Stegmuller J, Konishi Y, Huynh MA, Yuan Z, Dibacco S, Bonni A (2006) Cell-intrinsic regulation of axonal morphogenesis by the Cdh1-APC target SnoN. Neuron 50: 389-400

Stern CD (2001) Initial patterning of the central nervous system: how many organizers? Nat Rev Neurosci 2: 92-98

Stitt TN, Hatten ME (1990) Antibodies that recognize astrotactin block granule neuron binding to astroglia. Neuron 5: 639-649

Stroschein SL, Wang W, Zhou S, Zhou Q, Luo K (1999) Negative feedback regulation of TGFbeta signaling by the SnoN oncoprotein. Science 286: 771-774 
Sudakin V, Ganoth D, Dahan A, Heller H, Hershko J, Luca FC, Ruderman JV, Hershko A (1995) The cyclosome, a large complex containing cyclin-selective ubiquitin ligase activity, targets cyclins for destruction at the end of mitosis. Mol Biol Cell 6: 185-197

Susin SA, Lorenzo HK, Zamzami N, Marzo I, Snow BE, Brothers GM, Mangion J, Jacotot E, Costantini P, Loeffler M, Larochette N, Goodlett DR, Aebersold R, Siderovski DP, Penninger JM, Kroemer G (1999) Molecular characterization of mitochondrial apoptosis-inducing factor. Nature 397: 441-446

Suzuki Y, Imai Y, Nakayama H, Takahashi K, Takio K, Takahashi R (2001) A serine protease, HtrA2, is released from the mitochondria and interacts with XIAP, inducing cell death. Mol Cell 8: 613-621

Sweet ES, Previtera ML, Fernandez JR, Charych EI, Tseng CY, Kwon M, Starovoytov V, Zheng JQ, Firestein BL (2011) PSD-95 alters microtubule dynamics via an association with EB3.J Neurosci 31: 1038-1047

Tahirovic S, Bradke F (2009) Neuronal polarity. Cold Spring Harb Perspect Biol 1: a001644

Takahashi R, Imai Y, Hattori N, Mizuno Y (2003) Parkin and endoplasmic reticulum stress. Ann N Y Acad Sci 991: 101-106

Tanahashi H, Tabira T (1999) Genome structure and chromosomal mapping of the gene for Fe65L2 interacting with Alzheimer's beta-amyloid precursor protein. Biochem Biophys Res Commun 258: 385-389

Tao J, Rolls MM (2011) Dendrites have a rapid program of injury-induced degeneration that is molecularly distinct from developmental pruning. J Neurosci 31: 5398-5405

Taylor JP, Hardy J, Fischbeck KH (2002) Toxic proteins in neurodegenerative disease. Science 296: 1991-1995

Tessier-Lavigne M, Goodman CS (1996) The molecular biology of axon guidance. Science 274: 1123-1133

Trommsdorff M, Borg JP, Margolis B, Herz J (1998) Interaction of cytosolic adaptor proteins with neuronal apolipoprotein E receptors and the amyloid precursor protein. $J$ Biol Chem 273: 33556-33560

Tropea M, Johnson MI, Higgins D (1988) Glial cells promote dendritic development in rat sympathetic neurons in vitro. Glia 1: 380-392

Tuoc TC, Stoykova A (2008) Trim11 modulates the function of neurogenic transcription factor Pax6 through ubiquitin-proteosome system. Genes Dev 22: 1972-1986

Tuoc TC, Stoykova A (2010) Roles of the ubiquitin-proteosome system in neurogenesis. Cell Cycle 9: 3174-3180 
Urbanska M, Blazejczyk M, Jaworski J (2008) Molecular basis of dendritic arborization. Acta Neurobiol Exp (Wars) 68: 264-288

Verhagen AM, Ekert PG, Pakusch M, Silke J, Connolly LM, Reid GE, Moritz RL, Simpson RJ, Vaux DL (2000) Identification of DIABLO, a mammalian protein that promotes apoptosis by binding to and antagonizing IAP proteins. Cell 102: 43-53

Vikis HG, Li W, Guan KL (2002) The plexin-B1/Rac interaction inhibits PAK activation and enhances Sema4D ligand binding. Genes Dev 16: 836-845

Vikis HG, Li W, He Z, Guan KL (2000) The semaphorin receptor plexin-B1 specifically interacts with active Rac in a ligand-dependent manner. Proc Natl Acad Sci U S A 97: 12457-12462

Volkmar FR, Greenough WT (1972) Rearing complexity affects branching of dendrites in the visual cortex of the rat. Science 176: 1445-1447

Vucic D, Dixit VM, Wertz IE (2011) Ubiquitylation in apoptosis: a post-translational modification at the edge of life and death. Nat Rev Mol Cell Biol 12: 439-452

Walczak H, Krammer PH (2000) The CD95 (APO-1/Fas) and the TRAIL (APO-2L) apoptosis systems. Exp Cell Res 256: 58-66

Welch MD, Mullins RD (2002) Cellular control of actin nucleation. Annu Rev Cell Dev Biol 18: $247-288$

Whatley BR, Li L, Chin LS (2008) The ubiquitin-proteasome system in spongiform degenerative disorders. Biochim Biophys Acta 1782: 700-712

Whitford KL, Marillat V, Stein E, Goodman CS, Tessier-Lavigne M, Chedotal A, Ghosh A (2002) Regulation of cortical dendrite development by Slit-Robo interactions. Neuron 33: 47-61

Williams DW, Truman JW (2005) Remodeling dendrites during insect metamorphosis. J Neurobiol 64: 24-33

Williamson A, Banerjee S, Zhu X, Philipp I, Iavarone AT, Rape M (2011) Regulation of ubiquitin chain initiation to control the timing of substrate degradation. Mol Cell 42: 744757

Williamson A, Wickliffe KE, Mellone BG, Song L, Karpen GH, Rape M (2009) Identification of a physiological E2 module for the human anaphase-promoting complex. Proc Natl Acad Sci US A 106: 18213-18218

Wills ZP, Mandel-Brehm C, Mardinly AR, McCord AE, Giger RJ, Greenberg ME (2012) The nogo receptor family restricts synapse number in the developing hippocampus. Neuron 73: 466-481 
Wirths O, Breyhan H, Cynis H, Schilling S, Demuth HU, Bayer TA (2009) Intraneuronal pyroglutamate-Abeta 3-42 triggers neurodegeneration and lethal neurological deficits in a transgenic mouse model. Acta Neuropathol 118: 487-496

Wirths O, Multhaup G, Bayer TA (2004) A modified beta-amyloid hypothesis: intraneuronal accumulation of the beta-amyloid peptide--the first step of a fatal cascade. J Neurochem 91: 513-520

Witte H, Bradke F (2008) The role of the cytoskeleton during neuronal polarization. Curr Opin Neurobiol 18: 479-487

Wolter KG, Hsu YT, Smith CL, Nechushtan A, Xi XG, Youle RJ (1997) Movement of Bax from the cytosol to mitochondria during apoptosis. J Cell Biol 139: 1281-1292

Wong RO, Ghosh A (2002) Activity-dependent regulation of dendritic growth and patterning. Nat Rev Neurosci 3: 803-812

Wu JI, Lessard J, Olave IA, Qiu Z, Ghosh A, Graef IA, Crabtree GR (2007) Regulation of dendritic development by neuron-specific chromatin remodeling complexes. Neuron 56: 94-108

Wu T, Merbl Y, Huo Y, Gallop JL, Tzur A, Kirschner MW (2010) UBE2S drives elongation of K11-linked ubiquitin chains by the anaphase-promoting complex. Proc Natl Acad Sci U S A 107: $1355-1360$

Xu Z, Sproul A, Wang W, Kukekov N, Greene LA (2006) Siah1 interacts with the scaffold protein POSH to promote JNK activation and apoptosis. J Biol Chem 281: 303-312

Yi JJ, Ehlers MD (2007) Emerging roles for ubiquitin and protein degradation in neuronal function. Pharmacol Rev 59: 14-39

Yoshikawa S, McKinnon RD, Kokel M, Thomas JB (2003) Wnt-mediated axon guidance via the Drosophila Derailed receptor. Nature 422: 583-588

Yu F, Yao H, Zhu P, Zhang X, Pan Q, Gong C, Huang Y, Hu X, Su F, Lieberman J, Song E (2007) let-7 regulates self renewal and tumorigenicity of breast cancer cells. Cell 131: 1109-1123

Yu SW, Wang H, Poitras MF, Coombs C, Bowers WJ, Federoff HJ, Poirier GG, Dawson TM, Dawson VL (2002) Mediation of poly(ADP-ribose) polymerase-1-dependent cell death by apoptosis-inducing factor. Science 297: 259-263

Yu X, Malenka RC (2003) Beta-catenin is critical for dendritic morphogenesis. Nat Neurosci 6: $1169-1177$

Yuan J, Lipinski M, Degterev A (2003) Diversity in the mechanisms of neuronal cell death. Neuron 40: 401-413

Zambrano N, Bimonte M, Arbucci S, Gianni D, Russo T, Bazzicalupo P (2002) feh-1 and apl1, the Caenorhabditis elegans orthologues of mammalian Fe65 and beta-amyloid precursor 
protein genes, are involved in the same pathway that controls nematode pharyngeal pumping. J Cell Sci 115: 1411-1422

Zha H, Fisk HA, Yaffe MP, Mahajan N, Herman B, Reed JC (1996) Structure-function comparisons of the proapoptotic protein Bax in yeast and mammalian cells. Mol Cell Biol 16: 6494-6508

Zhang Y, McLaughlin R, Goodyer C, LeBlanc A (2002) Selective cytotoxicity of intracellular amyloid beta peptide1-42 through p53 and Bax in cultured primary human neurons. J Cell Biol 156: 519-529

Zheng N, Wang P, Jeffrey PD, Pavletich NP (2000) Structure of a c-Cbl-UbcH7 complex: RING domain function in ubiquitin-protein ligases. Cell 102: 533-539

Zhou D, Zambrano N, Russo T, D'Adamio L (2009) Phosphorylation of a tyrosine in the amyloid-beta protein precursor intracellular domain inhibits Fe65 binding and signaling. $J$ Alzheimers Dis 16: 301-307

Zhu H, Hummel T, Clemens JC, Berdnik D, Zipursky SL, Luo L (2006) Dendritic patterning by Dscam and synaptic partner matching in the Drosophila antennal lobe. Nat Neurosci 9: 349-355

Zimmerman LB, De Jesus-Escobar JM, Harland RM (1996) The Spemann organizer signal noggin binds and inactivates bone morphogenetic protein 4. Cell 86: 599-606

Zoghbi HY, Orr HT (2000) Glutamine repeats and neurodegeneration. Annu Rev Neurosci 23: $217-247$

Zou H, Henzel WJ, Liu X, Lutschg A, Wang X (1997) Apaf-1, a human protein homologous to C. elegans CED-4, participates in cytochrome c-dependent activation of caspase-3. Cell 90: 405-413

Zou H, Li Y, Liu X, Wang X (1999) An APAF-1.cytochrome c multimeric complex is a functional apoptosome that activates procaspase-9.J Biol Chem 274: 11549-11556 


\section{Curriculum Vitae}

\section{Annika Matz}

Kurze Straße 3

37073 Göttingen

Email: matz@em.mpg.de

Phone: 0176/61984726

Date of birth:

18.05.1984

Place of birth:

Wilhelmshaven

Nationality:

German

\section{Education}

2003

2003 to 2008

Since 09/2008-current
Käthe-Kollwitz Gymnasium, Wilhelmshaven Abitur (final grade: 2,1)

Georg-August University Göttingen

Diploma in Biology;

Major in biochemistry

Minors in chemistry and microbiology

Max-Planck-Institute for Experimental Medicine, Göttingen; PhD work in the group of Dr. Judith Stegmüller

\section{Research}

$10 / 2007$ to $06 / 2008$

Max-Planck-Institute for Experimental Medicine, Göttingen

AG Sereda, Neurogenetics Diploma thesis: „Effekte des Endothelin/Endothelin Rezeptor Typ B auf die Myelinisierung im peripheren Nervensystem“

Since 09/2008-current

PhD-project: The role of RNF157 in Central Nervous System development 
